P,NL-422/ Vol6

NUREG/CR-2850

PNL-4221

Vol. 6

\title{
Population Dose Commitments Due to Radioactive Releases from Nuclear Power Plant Sites in 1984
}

Prepared by D. A. Baker

Pacific Northwest Laboratory

Operated by

Battelle Memorial Institute

Prepared for

U.S. Nuclear Regulatory

Commission 


\section{NOTICE}

This report was prepared as an account of work sponsored by an agency of the United States Government. Neither the United States Government nor any agency thereof, or any of their employees, makes any warranty, expressed or implied, or assumes any legal liability of re. sponsibility for any third party's use, or the results of such use, of any information, apparatus, product or process disclosed in this report, or represents that its use by such third party would not infringe privately owned rights.

\section{NOTICE}

Availability of Reference Materials Cited in NRC Publications

Most documents cited in NRC publications will be available from one of the following sources:

1. The NRC Public Document Room, 1717 H Street, N.W. Washington, DC 20555

2. The Superintendent of Documents, U.S. Government Printing Office, Post Office Box 37082 , Washington, DC 20013-7082

3. The National Technical Information Service, Springfield, VA 22161

Although the listing that follows represents the majority of documents cited in NRC publica Jns, it is not intended to be exhaustive.

Referenced documents available for inspection and copying for a fee from the NRC Public Docuer t Room include NRC correspondence and nternal NRC memoranda; NRC Office of I spection and Enforcement bulletins, circulars, information notices, inspection and investigation notices; Licensee Event Reports; vendor reports and correspondence; Commission papers; and applicant and licensee documents and correspondence.

The following documents in the NUREG series are available for purchase from the GPO Sales Program: formal NRC staff and contractor reports, NRC-sponsored conference proceedings, and NRC booklets and brochures. Also available are Regulatory Guides, NRC regulations in the Code of Federal Regulations, and Nuclear Regulatory Commission /ssuances.

Documents available from the National Technical Information Service include NUAEG series reports and technical reports prepared by other federal agencies and reports prepared by the Atomic Energy Commission, forerunner agency to the Nuclear Regulatory Commission.

Documents available from public and special technical libraries include all open literature items, such as books, journal and periodical articles, and transactions. Federal Register notices, federal and state legislation, and congressional reports can usually be obtained from these libraries.

Documents such as theses, dissertations, foreign reports and transtations, and non-NRC conference proceedings are available for purchase from the organization sponsoring the publication cited.

Single copies of NRC draft reports are available free, to the extent of supply, upon written request to the Division of Information Support Services, Distribution Section, U.S. Nuclear Regulatory Commission, Washington, DC 20555.

Copies of industry codes and standards used in a substantive manner in the NRC regulatory process are maintained at the NRC Library, 7920 Norfolk Avenue, Bethesda, Maryland, and are available there for reference use by the public. Codes and standards are usually copyrighted and may be purchased from the originating organization or, if they are American National Standards, from the American National Standards Institute, 1430 Broadway, New York, NY 10018. 
NUREG/CR-2850

PNL-4221

Vol. 6

\section{Population Dose Commitments \\ Due to Radioactive Releases \\ from Nuclear Power Plant Sites \\ in 1984}

Manuscript Completed: December 1987

Date Published: January 1988

Prepared by

D. A. Baker

Pacific Northwest Laboratory

Richland, WA 99352

\section{Prepared for}

Office of Administration and Resources Management

U.S. Nuclear Regulatory Commission

Washington, DC 20555

NRC FIN B2243 


\section{PREVIOUS REPORTS IN THIS SERIES}

1. Population Dose Commitments Due to Radicactive Releases from Nuctear Power Plant Sites in 1975, PNL-2439, October 1977.

2. Population Dose Commitments Due to Radioactive Releases from Nuclear Power Plant Sites in 1976, NUREG/CR-1125, PNL-2940, December 1979.

3. Population Dose Commitments Due to Radioactive Releases from Nuclear Power Plant Sites in 1977, NUREG/CR-1498, PNL-3324, October 1980.

4. Population Dose Commitments Due to Radioactive Releases from Nuclear Power Plant Sites in 1978, NUREG/CR-2201, PNL-4039, June 1982.

5. Population Dose Commitments Due to Radioactive Releases from Nuclear Power PTant Sites in 1979, NUREG/CR-2850, PNL-4221, Vol. 1, December 1982.

6. Population Dose Commitments Due to Radioactive Releases from Nuclear Power Plant Sites in 1980, NUREG/CR-2850, PNL-4221, Vol. 2, August 1983.

7. Population Dose Commitments Due to Radioactive ReTeases from Nuclear Power Plant Sites in 1981, NUREG/CR-2850, PNL-4221, Vo1. 3, January 1985.

8. Population Dose Commitments Due to Radioactive Releases from NucTear Power PTant Sites in 1982, NUREG/CR-2850, PNL-4221, Vol. 4, June 1986.

9. Population Dose Commitments Due to Radioactive Releases from Nuctear Power Plant Sites in 1983, NUREG/CR-285D, PNL-4221, Vol. 5, January 1987. 


\section{ABSTRACT}

Population radiation dose commitments have been estimated from reported radionuclide releases from commercial power reactors operating during 1984. Fifty-year dose commitments from a one-year exposure were calculated from both liquid and atmospheric releases for four population groups (infant, child, teen-ager and adult) residing between 2 and $80 \mathrm{~km}$ from each of 56 sites. This report tabutates the results of these calculations, showing the dose commitments for both liquid and airborne pathways for each age group and organ. Also included for each of the sites is a histogram showing the fraction of the total population within 2 to $80 \mathrm{~km}$ around each site receiving various average dose commitments from the airborne pathways.

The total dose commitments (from both liquid and airborne pathways) for each site ranged from a high of 110 person-rem to a low of 0.002 person-rem for the sites with plants operating throughout the year with an arithmetic mean of 5 person-rem. The total population dose for al1 sites was estimated at 280 person-rem for the 100 million people considered at risk.

The site average individuat dose commitment from all pathways ranged from a low of $6 \times 10^{-6}$ mrem to a high of 0.04 mrem. No attempt was made in this study to determine the maximum dose commitment received by any one individual from the radionuclides released at any of the sites. 



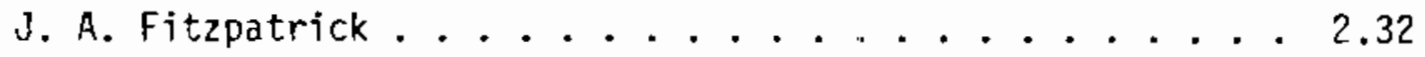

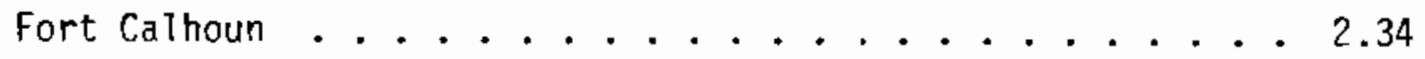

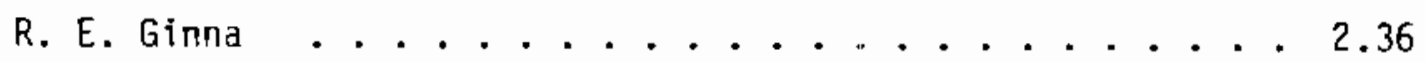

Grand GuTf . . . . . . . . . . . . . 2. . 2.38

Haddam Neck ................ . 2.40

Edwin I. Hatch $1 \& 2 \ldots \ldots . \ldots . \ldots . . \ldots 2 . \ldots$

Indian Point $1,2, \& 3 \ldots \ldots . \ldots . \ldots 2.44$

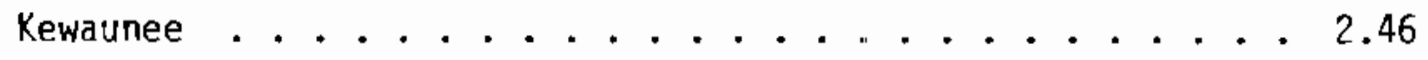

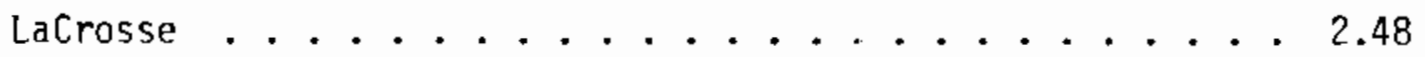

LaSatle $1 \& 2 \ldots \ldots \ldots . \ldots \ldots$

Limerick . . . . . . . . . . . . . 2.52

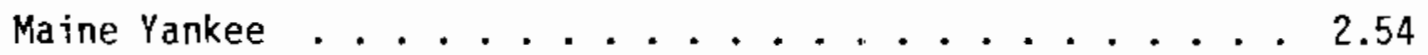

McGuire $1 \& 2 \ldots \ldots . \ldots \ldots$

Millstone $1 \& 2 \ldots \ldots \ldots \ldots . \ldots \ldots$

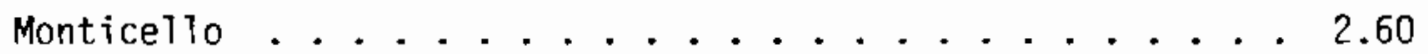

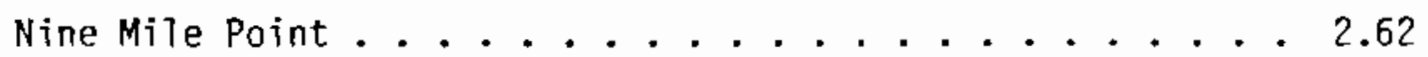

North Anna $1 \& 2 \ldots \ldots . \ldots . \ldots . . \ldots 2 . \ldots$

Oconee $1,2 \& 3 \ldots \ldots \ldots \ldots \ldots$

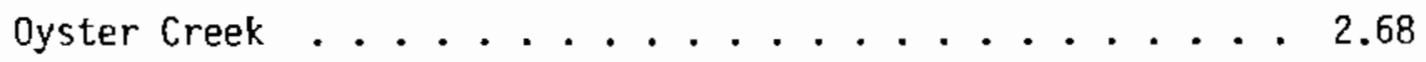

Palisades . . . . . . . . . . . . . 2.70

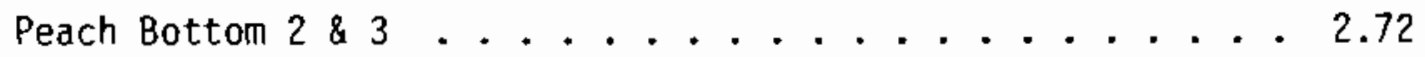

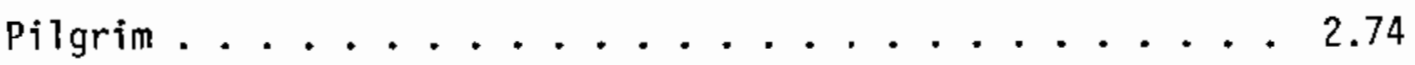

Point Beach $1 \& 2 \ldots \ldots . \ldots \ldots . \ldots . \ldots . \ldots$ 


\section{CONTENTS}

ABSTRACT ..................................... ACKNOWLEDGMENTS ....................... ix

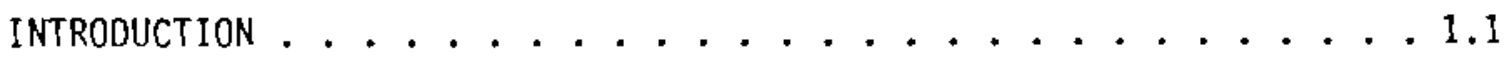
Site-Dependent Parameters . . . . . . . . . . 1.4

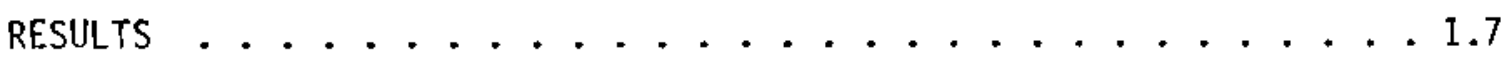

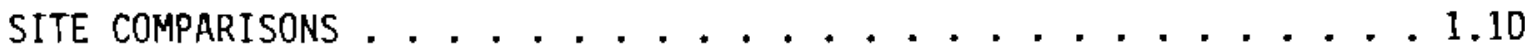

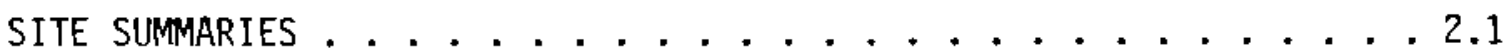

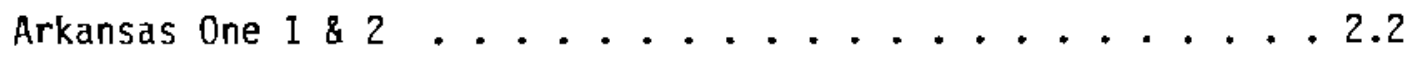

Beaver Valley ..................... . . 2.4

Big Rock Point . . . . . . . . . . . . . 2.6

Browns Ferry 1, 2, \& 3 . . . . . . . . . . 2.8

Brunswick I \& 2 . . . . . . . . . . . 2.10

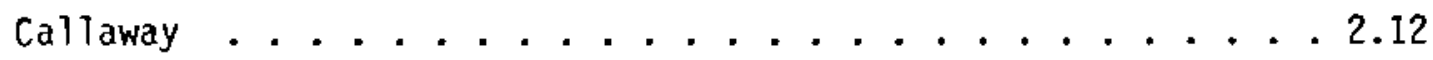

Calvert Cliffs $1 \& 2$ \& . . . . . . . . . 2.14

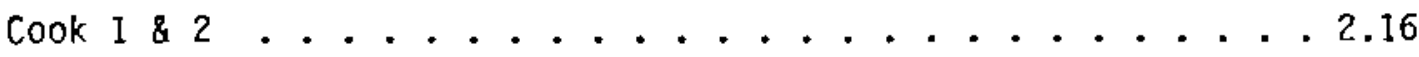

Cooper ........................ 2... 18

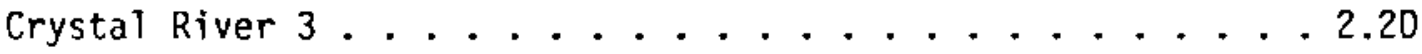

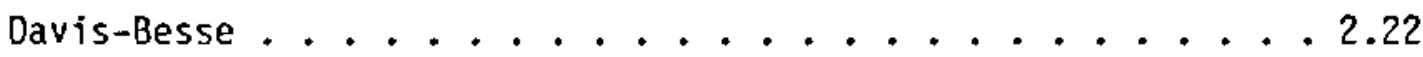

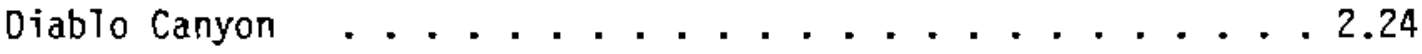

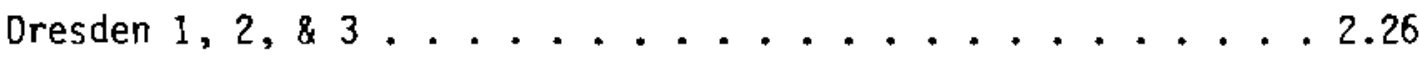

Duane Arnold . . . . . . . . . . . . . . . 2. 28

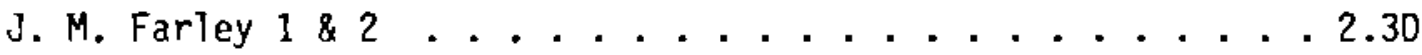


Prairie Island $1 \& 2$. . . . . . . . . . . . 2.78

Quad Cities $1 \& 2 \ldots . . . . . . . . . . . .2 .80$

Rancho Seco.................... 2. 2.82

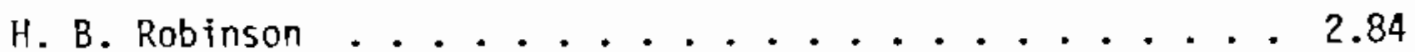

St. Lucie I\& 2 . . . . . . . . . . . . 2.86

Salem $1 \& 2$. . . . . . . . . . . . . 2. 2.88

San Onofre 1, 2,\&3 ............... 2...90

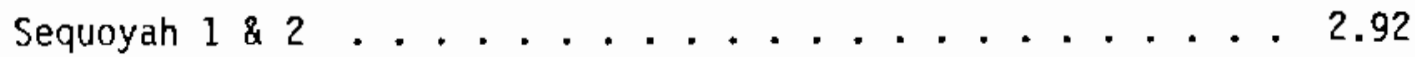

Summer .................... 2... 2. . . . . . . .

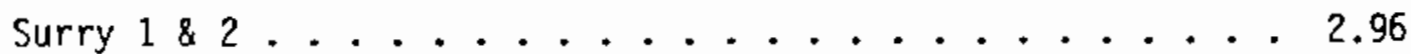

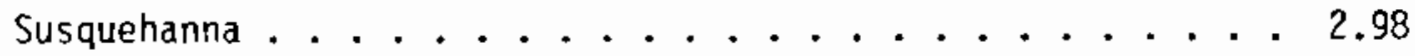

Three Mile Island I\& $2 . . . . . . . . . . . . .2 .100$

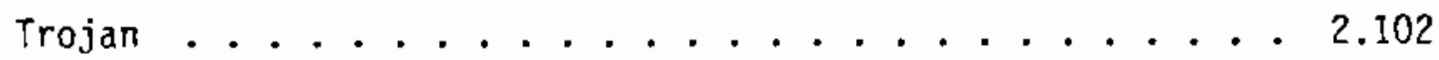

Turkey Point $3 \& 4$ \&............... 2. . . . . . . . . . 404

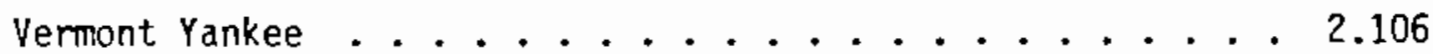

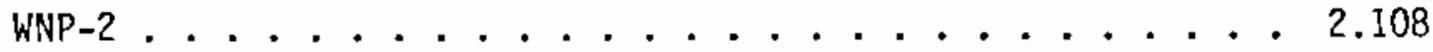

Yankee Rowe ................... 2. . . . . . . . 110

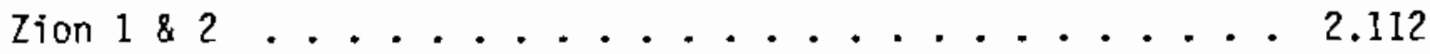

REFERENCES . . . . . . . . . . . . . . . . . . 3.1

APPENDIX ......................... . . . . . . . . 



\section{ACKNOWLEDGMENTS}

The author greatly appreciates the development of the population distributions around each site from census data by Anton A. Sinisgalli, A. R. Brauner, and Donald P. Cleary of the U.S. Nuclear Regulatory Commission. The author also gratefully acknowledges the contributions and suggestions by Richard A. Hartfield and Kazimieras Campe, also of the U.S. Nuclear Regulatory Commission. 
Most commercial nuclear power reactors release small amounts of radioactive materials to the environment during normal operation. Because of these releases, concern was expressed about the magnitude of the collective dose received by the general population residing around these nuclear power plants, In response to this concern, the Pacific Northwest Laboratory (PNL) ${ }^{(a)}$ contracted with the Nuclear Regutatory Commission (NRC) to undertake a series of studies to estimate radiation dose commitments produced by radionuclide releases from commercial light-water power reactors during the years 1975 through 1984 (see previous reports in this series, p. ii). This document is a continuation of these studies and considers the doses from releases during 1984 . In this study, as in those previous, we estimated the collective (population) dose commitment ${ }^{(b)}$ from both the liquid and gaseous releases to four age groups making up the population residing in the region of the site: infant ( 0 to $1 \mathrm{yr}$ ), child ( 1 to $11 \mathrm{yr}$ ), teen-ager ( 11 to $17 \mathrm{yr}$ ), and adult (17 yr and older).

The particular organs of reference in this study are listed in Table 1. The major pathways by which radionuciides travel from the reactor to the individual receptors are shown in Table 2 . Other possible liquid pathways such as direct exposure from waterborne activities (swimming, boating, shoreline recreation) and internal exposure through ingestion of food produced using contaminated irrigation water were not included; we have found from past experience that the doses from these pathways are generally much smaller than the doses from the pathways considered in this study.

The "source terms" used to estimate dose commitments produced from each site were the annual measured releases of radioactive materials as reported to the NRC by the plant operators and subsequently published in an NRC public document (TichTer, et a1. 1987).

The regional population for which we estimated dose commitments included those persons estimated to be living in a region between 2 and

(a) Operated by Battelle Memorial Institute for the Department of Energy.

(b) As used in this report, dose commitment describes the total-body dose equivalent in rem ( 1 rem $=0.01$ sievert) received over 50 years from intake during the year in which radioactive materials were released into the environment from the plants. 
TABLE 1. Organs Considered in This Study

Organs Affected by

Airborne Releases

Totat body

Thyroid

Bone

GI tract

Liver

Lung
Organs Affected by Waterborne Releases

Tota1 body

Thyroid

Bone

GI tract

Liver

TABLE 2. Pathways Considered in This Study by which Radionuctides Travel from Reactors to Persons

Pathways for

Airborne Releases

Air submersion

Contaminated ground

Inhalation

Ingestion of food crops

and animal products
Pathways for Waterborne Releases

Ingestion of drinking water

Ingestion of fish and invertebrates

$80 \mathrm{~km}$ around the reactor sites during 1984. Population distributions were supplied by the NRC's Office of Nuclear Reactor Regulation. Atmospheric transport factors (annual average dilution and annual average deposition) were calculated for the region around each site using appropriate meteorological data supplied by the NRC's 0ffice of Nuclear Reactor Regulation. To calculate the doses, we used models approved by the NRC. We incorporated these models into two small computer codes to expedite the dose calculations involved for each site.

Site-specific parameters other than releases, meteorology, and population were obtained from environmental statements [both final (FES) and draft (OES)] for the various reactors when avaiTable (Table 3). Such parameter values include the total population drinking contaminated water, river flow, dilution flow from the reactors (for sites not on rivers), fish and invertebrate harvest for region, and dilution factors for drinking water and aquatic foods. In those cases in which sitespecific data are not readily available and the particular pathway is not expected to result in a large dose, conservative assumptions have been used to estimate doses. The use of more realistic data should result in lower dose estimates in some cases. 


\section{TABLE 3. Environmental Statements for Power Plants Included in This Study}

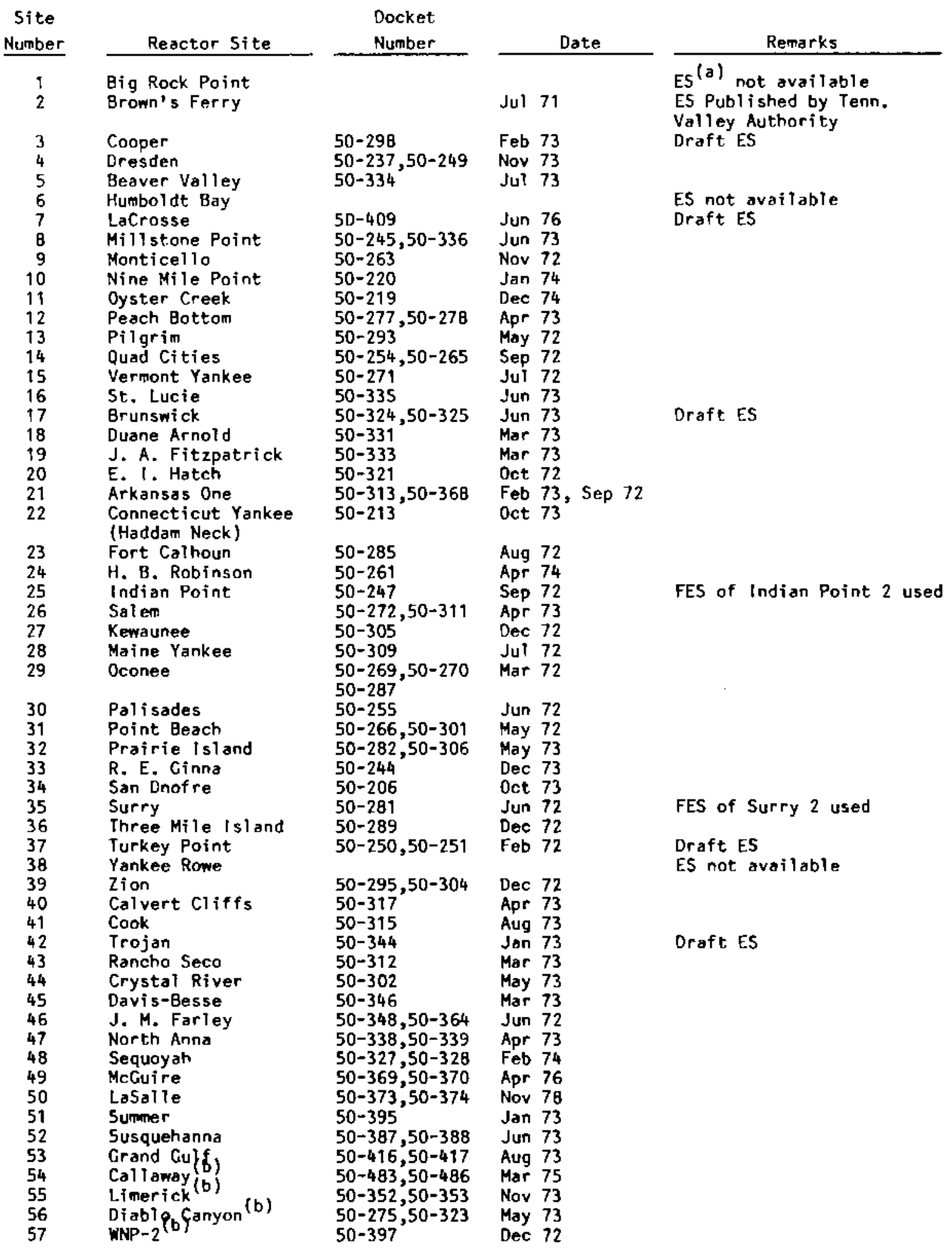

(a) Environmental Statement.

(b) Sites added for 1984 . 
The reactors included in this study, their type, Ticensed thermal power rating and net electrical output for 1984 are listed in Table 4 . Populations at risk and the dose comitments derived in the study are atso shown in this table.

\section{Site-Dependent Parameters}

In the Site Sumaries section, the location (including latitude and longitude) for each reactor site and the estimated 1984 population within 2 to $80 \mathrm{~km}$ around each site is given. In addition, the locations of major metropolitan centers within $80 \mathrm{~km}$ are listed along with their 1984 extrapolated populations. The populations of the Standard Metropolitan Statistical Areas (SMSA) are given where applicable. Next, the sitespecific data pertinent to the airborne pathways are specified. The average production rates of vegetable crops and animal products are given for the area within an $80-k m$ radius based upon the statewidz average. This production has been reduced for sites on lakes and seacoasts to account for the presence of the body of water. An animal grazing factor is estimated for each site location. This actor accounts for the fraction of the year during which grazing animals such as milk cows and beef cattle graze on fresh pasture in the region around the site. After average production rates are given, the period of record and the percent data recovery of the meteorological data used in calculating diffusion factors are indicated.

Various site-dependent factors associated with the waterborne pathways are presented next. For lake and ocean sites, we used the average dilution of plant effluents for the year 1984 specified by Tichler, et al. (1987). For river sites, the average annual river flow is tabulated. This flow was used in place of a dilution flow from the plant to account for dilutions of liquid releases at the locations of probable intake of drinking water and aquatic food cattch. Any exceptions to this scheme have been footnoted. Next is shown the estimated 1984 population utilizing drinking water drawn from supplies containing diluted effluents from the site. These are shown with an estimated dilution factor where applicable. Fish and invertebrate catch data taken from the respective plant environmental statement, when available (see Table 3), are listed next, along with estimated dilution factors For the lake and ocean sites. When site-specific fish and invertebrate catch data were not available, the generic consumption rates were used for the particular site. Sites on salt water were assumed to contribute no dose from drinking water. Any invertebrate catch from fresh water was not considered. 


\section{TABLE 4. Reactor Characteristics and Population Total-Body Dose Conmitments, 1984}

\begin{tabular}{|c|c|c|c|c|c|c|c|c|c|}
\hline \multirow[b]{2}{*}{ Site } & \multirow[b]{2}{*}{ Unit } & \multirow[b]{2}{*}{ Type } & \multirow{2}{*}{$\begin{array}{l}\text { Licensed } \\
\text { Thermal } \\
\text { Prower } \\
\text { (Mw) } \\
\end{array}$} & \multirow{2}{*}{$\begin{array}{c}\text { Electric } \\
\text { Energy } \\
\text { Ceneration } \\
1984 \text { (a) } \\
\text { (Tw,hr }{ }^{\text {Th }} \\
\end{array}$} & \multicolumn{3}{|c|}{$\begin{array}{c}\text { Pnputation Dose Commitment } \\
\text { [person-rtem] }\end{array}$} & \multirow[b]{2}{*}{$\begin{array}{l}\text { Peipulintion } \\
\text { at: Risk } \\
\end{array}$} & \multirow{2}{*}{$\begin{array}{c}\text { Average } \\
\text { Individual } \\
\text { Tatal-Body } \\
\text { Dose } \\
\text { Commitment } \\
\text { (mrem\} }\end{array}$} \\
\hline & & & & & Liquid & Air & Total & & \\
\hline $\begin{array}{l}\text { Arkansas One } \\
\text { Arkansas One }\end{array}$ & $3^{1}$ & $\begin{array}{l}\text { PWR } \\
\text { PWR }\end{array}$ & $\begin{array}{l}2568 \\
2815 \\
5383\end{array}$ & $\begin{array}{l}4.60 \\
6.20 \\
10.8\end{array}$ & 3.7 & 0.05 & 3.8 & $1.9 E 5$ & $2,0 \mathrm{E}-2$ \\
\hline Beaver Valley & $\uparrow$ & PWR & 2657 & 4.75 & 0.048 & 0.14 & 0.19 & 3. $6 \mathrm{EE} 6$ & $5.2 \mathrm{E}-5$ \\
\hline Big Rock Point (b) & l & BWR & 240 & 0.47 & 0.48 & 4.0 & 4.5 & $1.7 \mathrm{ES}$ & $2.7 \mathrm{E}-2$ \\
\hline $\begin{array}{l}\text { Browns Ferry } \\
\text { Growns Ferry }\end{array}$ & $\begin{array}{l}1 \\
2 \\
3 \\
\text { TOTAL. }\end{array}$ & $\begin{array}{l}\text { BWR } \\
\text { BWR } \\
\text { DWR }\end{array}$ & $\begin{array}{l}3293 \\
3293 \\
3293 \\
9879\end{array}$ & $\begin{array}{r}7.85 \\
4.04 \\
0.29 \\
12.18\end{array}$ & 17. & 89. & 110. & $7.3 \mathrm{ES}^{-}$ & $1.4 E-1$ \\
\hline $\begin{array}{l}\text { Brunswick } \\
\text { Brunswick }\end{array}$ & $\begin{array}{l}1 \\
2 \\
\text { TOTAL }\end{array}$ & $\begin{array}{l}\text { BWR } \\
\text { GWP? }\end{array}$ & $\begin{array}{l}2436 \\
2436 \\
4872\end{array}$ & $\begin{array}{l}5.03 \\
1.39 \\
6.42\end{array}$ & 0.0002 & 4.1 & 4.1 & $2.3 E 5$ & $1.8 E-2$ \\
\hline Callaray & 1 & PWR & 3411 & 0.32 & 0 & 0.0015 & 0.0015 & $3.6 \mathrm{ES}$ & $4.1 E-6$ \\
\hline $\begin{array}{l}\text { Calvert Cliffs } \\
\text { Calvert Clifts }\end{array}$ & $\begin{array}{l}1 \\
2 \\
\text { TOTALI. }\end{array}$ & $\begin{array}{l}\text { PWR } \\
\text { PWR }\end{array}$ & $\begin{array}{l}2700 \\
2700 \\
5400\end{array}$ & $\begin{array}{r}6.22 \\
5.34 \\
11.56\end{array}$ & 0.57 & 0.13 & 0.70 & $2.7 \mathrm{EG}$ & $2.6 E-4$ \\
\hline $\begin{array}{l}\text { Cook } \\
\text { Cook }\end{array}$ & $\stackrel{1}{2}_{\text {YOTAL }}$ & $\begin{array}{l}\text { PWR } \\
\text { PWP }\end{array}$ & $\begin{array}{l}3250 \\
3391 \\
6641\end{array}$ & $\begin{array}{r}7.75 \\
5.36 \\
13.7 !\end{array}$ & 0.29 & 0.053 & 0.34 & $1.1 \mathrm{EG}$ & $3.0 E-4$ \\
\hline Cooper & 1 & QWR & $238 t$ & 3.47 & 0.056 & 0.014 & 0.070 & $1.7 \in 5$ & $4.0 E-4$ \\
\hline Crystal River & 3 & PWR & 2452 & 6.48 & E.8 & 0.0090 & 6.8 & $4.1 \mathrm{E5}$ & $1.6 \mathrm{E}-2$ \\
\hline Davis-Busse & 1 & PWR & 2772 & 4.29 & 0.37 & 0.010 & 0.38 & $1.8 E 6$ & $2,1 E-4$ \\
\hline Diabto Canyon & 1 & PWR & $333 \mathrm{~B}$ & 0.20 & $1.6 E-5$ & $2.2 \mathrm{E}-5$ & $3 . B E-5$ & $2.8 E 5$ & $1.4 E-7$ \\
\hline Dresden & $\begin{array}{l}1 \\
2 \\
3 \\
\text { TOTAL }\end{array}$ & $\begin{array}{l}\text { BWR } \\
\text { BWR } \\
\text { BWR }\end{array}$ & $\begin{array}{r}700 \\
2527 \\
2527 \\
5754\end{array}$ & $\begin{array}{l}4.46 \\
2.11 \\
2.11 \\
8.68\end{array}$ & 0 & 1.1 & 1.1 & $6.4 E 6$ & $1.7 E-4$ \\
\hline Duane arnold & 1 & BWR & 1638 & 2.72 & $4.4 E-9$ & 0.033 & 0.0332 & 6.005 & $5.4 E-5$ \\
\hline $\begin{array}{l}\text { J, K, Farley } \\
\text { J. M. Farley }\end{array}$ & $\begin{array}{l}1 \\
2 \\
\text { TOTAL. }\end{array}$ & $\begin{array}{l}\text { PWR } \\
\text { PWR }\end{array}$ & $\begin{array}{l}2652 \\
2652 \\
5304\end{array}$ & $\begin{array}{r}5.43 \\
6.62 \\
12.05\end{array}$ & 0.066 & 0.16 & 0.23 & $3.6 \mathrm{ES}$ & $6.2 \mathrm{E}-4$ \\
\hline J. A. Fitzratrick & 1 & $B W R$ & 2436 & 4.90 & 0.0017 & 0.97 & 0.97 & $8.5 \mathrm{ES}$ & $1.1 E-3$ \\
\hline Fort Calhour & 1 & PWR & 1420 & 2.33 & 1.9 & 0.017 & 1.9 & $7.6 \mathrm{E} 5$ & $2,5 \mathrm{E}-3$ \\
\hline R. E, Ginna & 1 & PWR & 1520 & 3.16 & 0.66 & 0.029 & 0.69 & 1.256 & $5.7 E-4$ \\
\hline Grand Gulf & 1 & Bwk & $3 B 33$ & 0.16 & $4 E-6$ & 0.0019 & 0.0019 & $3.3 \mathrm{ES}$ & $5.7 E-6$ \\
\hline Haddam Nerk & 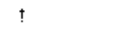 & F'WR & 1825 & 3.36 & 0.29 & 1.7 & 1.5 & $3.4 E 6$ & $4 \cdot 3 E-4$ \\
\hline $\begin{array}{l}\text { E. I. Hatch } \\
\text { E. 1. Hatch }\end{array}$ & ${ }^{t}$ TOTAL & $\begin{array}{l}\text { BWR } \\
\text { BWR }\end{array}$ & $\begin{array}{l}2436 \\
2436 \\
4872\end{array}$ & $\begin{array}{l}3.60 \\
1.06 \\
5.48\end{array}$ & 7.9 & 0.14 & 8.0 & 3.25 .5 & $7.5 \mathrm{E}-2$ \\
\hline $\begin{array}{l}\text { Indian Point } \\
\text { Indian Point }\end{array}$ & $\begin{array}{ll}1 & \\
2 & \\
3 & \\
& \text { TOTAL }\end{array}$ & $\begin{array}{l}\text { PWR } \\
\text { PWR } \\
\text { PWR }\end{array}$ & $\begin{array}{r}615 \\
2758 \\
2760 \\
6133\end{array}$ & $\begin{array}{l}\text { (1) } \\
2.89 \\
5.04 \\
9.93\end{array}$ & 0.60 & 2.6 & 3.2 & $1.5 \mathrm{E} 7$ & $2.1 t-4$ \\
\hline Kewaunee & 1 & FWR & 1650 & 3.81 & 0.55 & 0.0012 & 0.55 & $6.2 \mathrm{E5}$ & $B, 9 E-4$ \\
\hline Lacrosse $e^{(b)}$ & 1 & AWR & 165 & 0.32 & 0.42 & 0.64 & 1.1 & 3. SES & $3,1 \mathrm{E}-3$ \\
\hline $\begin{array}{l}\text { La5a17a } \\
\qquad \text { LaSalle }\end{array}$ & $\begin{array}{ll}1 & \\
2 & \\
& \text { TOTAL }\end{array}$ & $\begin{array}{l}\text { BWR } \\
\text { GWR }\end{array}$ & $\begin{array}{l}3323 \\
3293 \\
6616\end{array}$ & $\begin{array}{l}5.2 \\
1.4 \\
6.5\end{array}$ & 0 & 0.027 & 0.027 & $1.0 \mathrm{E} 6$ & $\begin{array}{l}1,4 E-6 \\
2.6 E-5\end{array}$ \\
\hline Limerick & & BWR & 3293 & 0 & 0.0002 & 0 & 0.0002 & $6.8 \mathrm{~F} 6$ & $2.4 E-8$ \\
\hline Maine Yankee & 1 & PWR & 2440 & 5.13 & 0.0006 & 0.0056 & 0.0062 & $6.1 \mathrm{ES}$ & 1.0E-5 \\
\hline $\begin{array}{l}\text { MacGuire } \\
\text { McCuire }\end{array}$ & $\begin{array}{l}1 \\
2 \text { TOIAL }\end{array}$ & $\begin{array}{l}\text { PWR } \\
\text { PWK }\end{array}$ & $\begin{array}{l}3411 \\
3411 \\
5822\end{array}$ & $\begin{array}{r}6.42 \\
6.56 \\
12.98\end{array}$ & 20. & 0.17 & 20. & $1.7 E 6$ & $1.2 \mathrm{E}-2$ \\
\hline $\begin{array}{l}\text { Milistione } \\
\text { Milistone }\end{array}$ & $\begin{array}{ll}1 \\
2 \\
& \\
& \end{array}$ & $\begin{array}{l}\text { BWK } \\
\text { PWK }\end{array}$ & $\begin{array}{l}2011 \\
2560 \\
4571\end{array}$ & $\begin{array}{r}4.32 \\
6.61 \\
10.93\end{array}$ & 0.03 & 1.3 & 1.3 & $2.5 E 6$ & $5.2 \mathrm{E}-4$ \\
\hline Honticello & 1 & BikR & 1670 & 0.26 & 0 & 0.049 & 0.049 & $2,2 \mathrm{E} 6$ & $2+2 E-5$ \\
\hline Nine Mile Point & 1 & $\mathrm{BwK}$ & 1850 & 3.6 & 0 & 0.029 & 0.029 & 8.5ES & $3.4 E-5$ \\
\hline
\end{tabular}


TABLE 4. (contd)

\begin{tabular}{|c|c|c|c|c|c|c|c|c|c|}
\hline \multirow[b]{2}{*}{ Site } & \multirow[b]{2}{*}{$\underline{\text { Wnit }}$} & \multirow[b]{2}{*}{ Type } & \multirow{2}{*}{$\begin{array}{l}\text { Licensed } \\
\text { Thermal } \\
\text { Power } \\
\text { (Mw) } \\
\end{array}$} & \multirow{2}{*}{$\begin{array}{c}\text { Electric } \\
\text { Energy } \\
\text { Generation } \\
1984 \text { (a) } \\
\text { (Tw,hr) }\end{array}$} & \multicolumn{3}{|c|}{$\begin{array}{c}\text { Posulation Dose Compitment } \\
\text { (person-rem) }\end{array}$} & \multirow{2}{*}{$\begin{array}{l}\text { Population } \\
\text { at Risk } \\
\end{array}$} & \multirow{2}{*}{$\begin{array}{c}\text { Average } \\
\text { Individual } \\
\text { Total-Body } \\
\text { Dose } \\
\text { Commitment } \\
\text { (mrem) }\end{array}$} \\
\hline & & & & & Liguid & Air. & Total & & \\
\hline $\begin{array}{l}\text { North Anns } \\
\text { North Anno }\end{array}$ & $\begin{array}{ll}1 & \\
2 & \\
\text { TOTAL }\end{array}$ & $\begin{array}{l}\text { PWR } \\
\text { PWR }\end{array}$ & $\begin{array}{l}2775 \\
2775 \\
5550\end{array}$ & $\begin{array}{l}3.78 \\
4.72 \\
8.50\end{array}$ & 44. & 0.18 & 44. & $1.1 \mathrm{E} 6$ & $4.1 E-2$ \\
\hline $\begin{array}{l}\text { Oconee } \\
\text { Oconer }\end{array}$ & $\begin{array}{ll}1 & \\
2 & \\
3 & \\
& \text { TOTAL }\end{array}$ & $\begin{array}{l}\text { PWR } \\
\text { PWR } \\
\text { PWR }\end{array}$ & $\begin{array}{l}2568 \\
2568 \\
2568 \\
7704\end{array}$ & $\begin{array}{r}6.17 \\
7.30 \\
5.35 \\
18.82\end{array}$ & 4.1 & 0.56 & 4.7 & $9.5 E 5$ & $4,9 E-3$ \\
\hline Oyster Creek & 1 & BWR & 2930 & $0.2 B$ & 0.0329 & 1.8 & 1.8 & $3.6 E 6$ & $5.1 E-4$ \\
\hline Palisades & 1 & PWR & 2530 & $0.8 \mathrm{t}$ & 0.12 & 0.0014 & 0.12 & 1.066 & 1. $2 E-4$ \\
\hline $\begin{array}{l}\text { Peach Bottonn } \\
\text { Peach Bottom }\end{array}$ & $3_{3}^{2}$ TOTAL & $\begin{array}{l}\text { BWR } \\
\text { BWR }\end{array}$ & $\begin{array}{l}3293 \\
3293 \\
6586\end{array}$ & $\begin{array}{l}2.43 \\
7.45 \\
9.88\end{array}$ & 1.1 & 8.5 & 9.6 & $4.3 E 6$ & $2.2 E-3$ \\
\hline Pilgrim & 1 & BWR & 1998 & 0.004 & 0.04 & 0.011 & 0.051 & $4.3 E 6$ & $1.2 \varepsilon-5$ \\
\hline $\begin{array}{l}\text { Point Beach } \\
\text { Point Beach }\end{array}$ & $\begin{array}{l}1 \\
2 \\
\\
\end{array}$ TOTAE & $\begin{array}{l}\text { PWR } \\
\text { PWR }\end{array}$ & $\begin{array}{l}1518 \\
1518 \\
3036\end{array}$ & $\begin{array}{l}3.11 \\
3.51 \\
6.62\end{array}$ & 0.15 & 0.075 & 0.24 & $6.2 \mathrm{E5}$ & $3.8 E-4$ \\
\hline $\begin{array}{l}\text { Prairite |sland } \\
\text { Prairie Island }\end{array}$ & $\begin{array}{l}1 \\
2 \text { TOTAL }\end{array}$ & $\begin{array}{l}P W R \\
P Q R\end{array}$ & $\begin{array}{l}1650 \\
1650 \\
3300\end{array}$ & $\begin{array}{l}4.16 \\
3.97 \\
8.07\end{array}$ & 0.013 & 0.046 & 0.059 & $2.2 E 6$ & $2.7 \mathrm{E}+5$ \\
\hline $\begin{array}{l}\text { Quad Cities } \\
\text { Quad Cities }\end{array}$ & $\begin{array}{l}1 \\
2 \text { TOTAL }\end{array}$ & $\begin{array}{l}\text { EWRR } \\
B W R R\end{array}$ & $\begin{array}{l}2511 \\
2511 \\
5022\end{array}$ & $\begin{array}{l}3.35 \\
4.98 \\
8.33\end{array}$ & 0.037 & 0.53 & 0.57 & $7.2 \mathrm{ES}$ & $7.8 E-4$ \\
\hline Rancho Seco & 1 & PWR & 2772 & 3.77 & 9.5 & 0.23 & 9.7 & $1.9 E 6$ & $5.1 E-3$ \\
\hline H. B. Robinson & 2 & PWR & 2200 & 0.190 & 0.26 & 0.0013 & 0.26 & $7.0 \mathrm{ES}$ & $3.7 \mathrm{E}-4$ \\
\hline $\begin{array}{l}\text { St. Lucie } \\
\text { St. Lucie }\end{array}$ & $\begin{array}{l}1 \\
2 \\
\\
\text { TOTAL }\end{array}$ & $\begin{array}{l}\text { PWR } \\
\text { PWR }\end{array}$ & $\begin{array}{l}2560 \\
2700 \\
5260\end{array}$ & $\begin{array}{l}4.23 \\
5.56 \\
9.79\end{array}$ & 0.0022 & 1.4 & 1.4 & $6.4 \mathrm{ES}$ & $2.2 \mathrm{E}-3$ \\
\hline $\begin{array}{l}\text { Solem } \\
\text { Salem }\end{array}$ & $\begin{array}{l}1 \\
2 \\
\\
\end{array}$ Total & $\begin{array}{l}\text { PWR } \\
\text { PWR }\end{array}$ & $\begin{array}{l}3338 \\
3338 \\
6676\end{array}$ & $\begin{array}{l}2.13 \\
3.20 \\
5.33\end{array}$ & 0.29 & 0.23 & 0.52 & $4.8 \mathrm{E} 6$ & $1.1 \varepsilon-4$ \\
\hline $\begin{array}{l}\text { San Onofre } \\
\text { San Onofre }\end{array}$ & $\begin{array}{l}1 \\
2 \\
3 \\
\\
\end{array}$ & $\begin{array}{l}\text { PWR } \\
\text { PWR } \\
\text { PWR }\end{array}$ & $\begin{array}{l}1347 \\
3410 \\
3390 \\
8147\end{array}$ & $\begin{array}{l}0.26 \\
5.27 \\
4.10 \\
9.63\end{array}$ & 1.7 & 2.1 & 3.8 & 5.056 & $7.5 E-4$ \\
\hline $\begin{array}{l}\text { Sequay ah } \\
\text { Sequoy ath }\end{array}$ & $\begin{array}{ll}1 & \\
2 & \\
& \text { TOTAL }\end{array}$ & $\begin{array}{l}\rho_{W R} \\
\rho_{N R}\end{array}$ & $\begin{array}{l}2815 \\
2815 \\
5630\end{array}$ & $\begin{array}{r}6.10 \\
6.40 \\
12.50\end{array}$ & 1.4 & 0.41 & 1.8 & $8.5 E 5$ & $2,9 E-3$ \\
\hline Sumner & 1 & PwR & 2775 & 4.20 & 0.54 & 0.0007 & 0.54 & B.6ES & $6.3 E-4$ \\
\hline $\begin{array}{l}\text { Surry } \\
\text { Surry }\end{array}$ & $\begin{array}{l}1 \\
2 \text { TOTAL }\end{array}$ & $\begin{array}{l}\text { PWR } \\
\text { PWR }\end{array}$ & $\begin{array}{l}2441 \\
2441 \\
4882\end{array}$ & $\begin{array}{l}3.33 \\
5.21 \\
8.54\end{array}$ & 1,6 & 0.32 & 1.9 & $1.8 E 6$ & $1,1 E-3$ \\
\hline $\begin{array}{l}\text { Susquehanna } \\
\text { Susquehanna }\end{array}$ & $\begin{array}{l}1 \\
2 \text { TOTAL }\end{array}$ & $\begin{array}{l}\text { BWR } \\
\text { BWR } \\
\text { BWR }\end{array}$ & $\begin{array}{l}3293 \\
3293 \\
6586\end{array}$ & $\begin{array}{l}6.09 \\
0.93 \\
7.02\end{array}$ & 0.0059 & 0.04 & 0.047 & $1.5 \mathrm{E} 6$ & $3.1 E-5$ \\
\hline $\begin{array}{l}\text { Three Mile Island } \\
\text { Three Mile Island }\end{array}$ & $\begin{array}{ll}1 & \\
2 & \\
& \text { TOTAL }\end{array}$ & $\begin{array}{l}P W R \\
P W R\end{array}$ & $\begin{array}{l}2535 \\
2772 \\
5307\end{array}$ & $\begin{array}{l}0 \\
0 \\
0\end{array}$ & 0.053 & 0.025 & 0.078 & $2.1 \mathrm{E} 6$ & $3.7 E-5$ \\
\hline Trojan & 1 & Pur & $341 \uparrow$ & 4.74 & 0.020 & 0.056 & 0.076 & $1.4 E 6$ & $5.4 E-5$ \\
\hline $\begin{array}{l}\text { Turkey Point } \\
\text { Turkey Point }\end{array}$ & ${ }^{3}$ TOTAL & $\begin{array}{l}P \mathbb{R} \\
P \mathbb{R}\end{array}$ & $\begin{array}{l}2200 \\
2200 \\
4400\end{array}$ & $\begin{array}{l}4.78 \\
3.08 \\
7.86\end{array}$ & $0 . \operatorname{co2} 7$ & 0.17 & 0.17 & $2.6 \mathrm{E} 6$ & $6.5 E-5$ \\
\hline Vermont Yankee & 1 & BWR & 1593 & 3.34 & 0 & 0.096 & 0.096 & $1.4 \mathrm{EG}$ & $7,0 E-5$ \\
\hline $\mathbb{N P}-2$ & & $B w R$ & 3323 & 0.41 & 0.1001 & 0.028 & 0.028 & $2.6 E 5$ & $1.1 E-4$ \\
\hline Yankee Rome & 1 & PWR & 600 & 1.03 & 0.27 & 0.21 & 0.48 & $1.6 E 6$ & $3.0 \mathrm{E}-4$ \\
\hline $\begin{array}{l}\text { Zion } \\
\text { Zion }\end{array}$ & $2^{1}$ & $\begin{array}{l}\text { PWR } \\
\text { PWR }\end{array}$ & $\begin{array}{l}3250 \\
3250 \\
6500\end{array}$ & $\begin{array}{r}5.69 \\
5.99 \\
11.68 \\
\end{array}$ & 33. & 0.69 & 34. & $7.2 \mathrm{E} 6$ & 4.7E-3 \\
\hline TOYAL FOR ALL SITES & & & & 320.80 & 160. & 120. & 280. & $1,1 E 8$ & $\cdots$ \\
\hline Arithmetic Mean & & & & 5.7 & 2.9 & 2.2 & 5.1 & 2.056 & $6.6 \mathrm{E}-3$ \\
\hline Geometric Mean & & & & 0.88 & 0.175 & 0.096 & 0.39 & $1.2 \mathrm{E} 6$ & $2.5 E-4$ \\
\hline
\end{tabular}

(a) $1 \mathrm{TW} \cdot \mathrm{he}=3.6 \mathrm{E} 15$ joules.

(b) Does not have charcoal delay beds in the gaseous effluent line from air ecector. 
RESULTS

This report consists of a sumary of values used for site-specific parameters at each site, as explained above, and the results of population dose commitment calculations. The population dose commitments are presented in two tables facing the page summarizing site-specific parameters for that site. These tables include both waterborne and airborne pathway dose commitments for the several organs of reference for each age group investigated. They also include the dose to the whole population, which includes all age gropps. The airborne population dose commitments for each of 160 segments partitioning the region around the site were divided by the population residing within that segment to derive an average individual dose for that segment. These doses are sumarized as a histogram showing percent of the population receiving a given dose level for each site. The fractional population dose from the waterborne pathway was not determined in this manner, because the NRC does not at present take into account the location of individuals exposed via this pathway, except those exposed through ingestion of drinking water.

Population dose commitments estimated for both the waterborne pathways and airborne pathways varied widely over the 56 sites (84 reactors) studied. The tota1 dose commitments (from both pathways) varied from a high of 110 to a low of 0.002 person-rem for plants in operation during the whole year. The arithmetic mean for the total dose from liquid pathways (2.9 person-rem) and airborne pathways (2.2 person-rem) was 5 person-rem (see Table 4).

As in past years, most of the plants accounted for less than a person-rem to their surrounding population from plant releases of radionuclides into liquid effluent streams. Oniy four sites had waterborne releases that resulted in population doses exceeding 10 person-rem. Those sites were Browns Ferry (17 person-rem), McGuire (20 person-rem), North Anna (44 person-rem) and Zion (33 person-rem). These waterborne doses were due almost totally to radioactive cesium.

The doses from radionuclide releases from plant vents and/or stacks into the atmosphere also accounted for less than a person-rem for most sites. Only one site had airborne releases resulting in 10 or more person-rem. This site was Browns Ferry ( 89 person-rem). These airborne doses were the result of krypton- 88 and its daughters.

(a) See Appendix for definition of segments. 
The total population dose commitments from all sites for 1984 were estimated to be 160 person-rem via waterborne pathways and 120 person-rem via airborne pathways (Table 4).

Figure 1 is a histogram for all 56 sites taken together. We can see from this plot that about $56 \%$ of the total population at risk (100 million) would each receive a dose comnitment of between $3 \times 10^{-5}$ and $3 \times 10^{-4}$ mrem. We can see further that about $2 \%$ receive a dose which is less than $1 \times 10^{-6}$ mrem. Although not discernible from the plot, $0.17 \%$ received a dose of between 0.1 and $10 \mathrm{mrem}$. However, no attempt was made in this study to estimate the maximum dose commitment received by any one individual from the radionuclicles released at any of the sites.

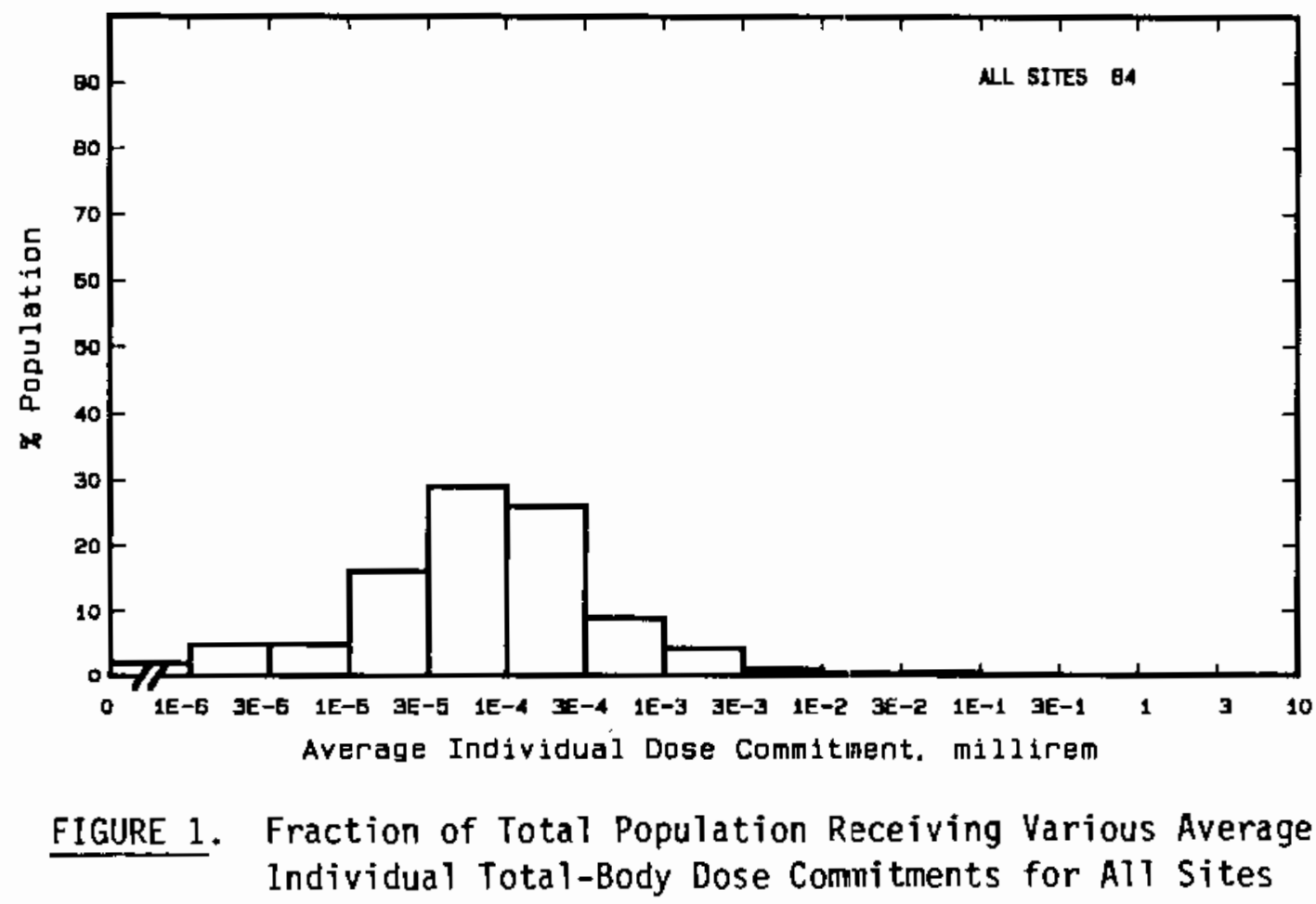

Figure 2 shows graphically in "Box-and-Whisker Plots" the wide range of the airborne population dose commitments for the reactor sites. The median, upper and lower quartiles and upper and lower octiles for the distribution of doses calculated for each of 160 segments are indicated for each site. The upper and lower octiles are shown as the horizontal bars on the "whiskers;" the upper and lower quartiles are indicated as the top and bottom of the "box." The median is indicated as the horizontal line inside the box. No airborne releases were reported for the Limerick Site for 1984; therefore, none are plotted. ATso, since Diablo Canyon was just started up this year, its airborne releases were extremely low. 


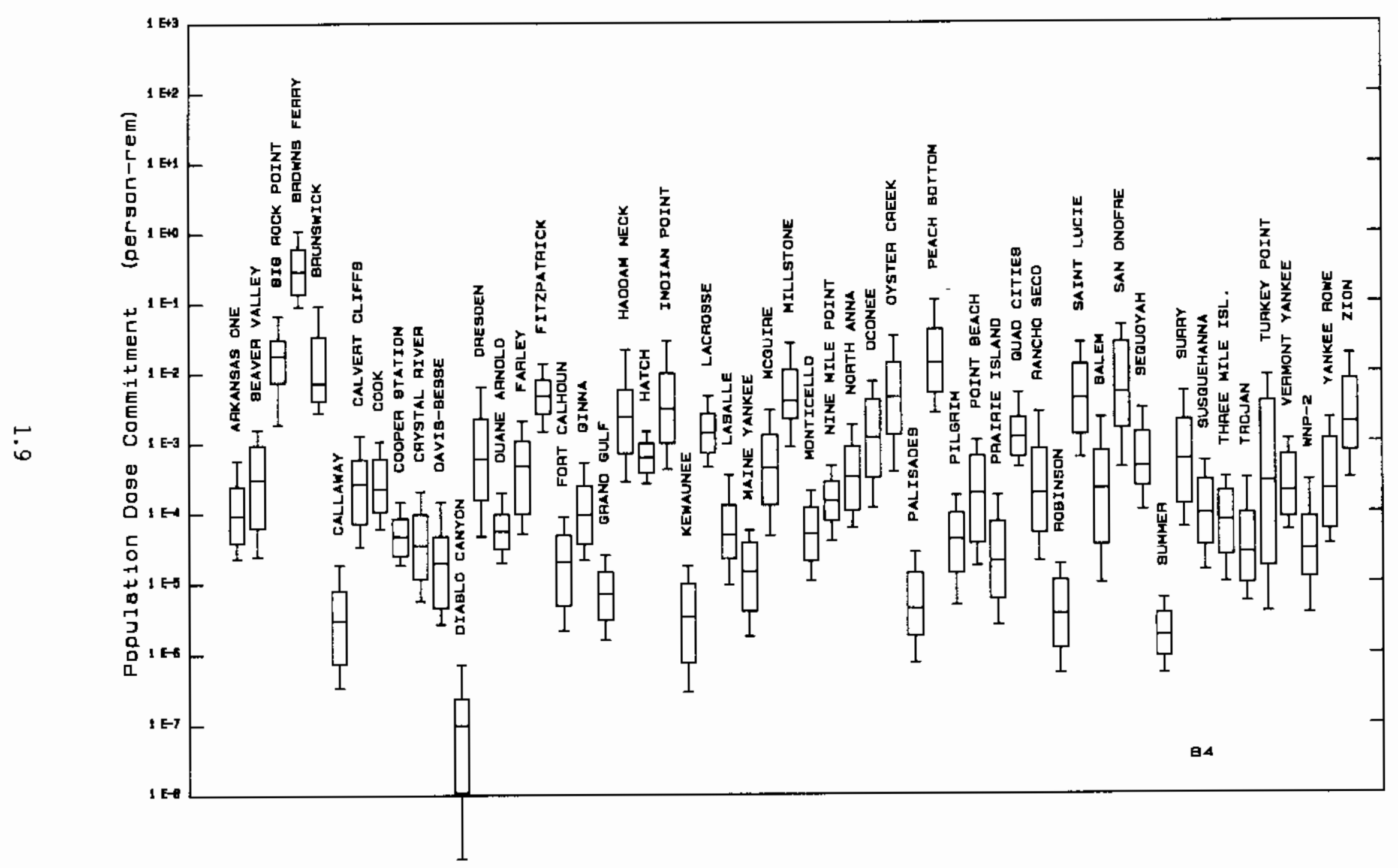

FIGURE 2. Airborne Population Dose Commitment for the Reactor Sites, 1984 
We should point out here, however, that the doses estimated in this study are extremely low compared to an average annual background dose of approximately 100 mrem. We have compared dose commitments calculated in this study with annual background. However, this comparison is not quite exact, since these dose commitments are those total-body doses received from the year's (1984) effluent release over 50 years of a person's lifetime. However, most of the dose commitment calculated here is delivered in the first year, so the comparison is reasonably valid.

For comparison purposes, the doses in the site summary tables are given to two significant figures; however, the data and models used to calculate the doses limit their accuracy to at most one significant figure. 


\section{SITE COMPARISONS}

Compared to 1983 , the total dose from waterborne pathways is higher (160 vs. 95), and also, the total dose from airborne pathways is higher (120 vs. 76). Table 5 compares the total population dose commitments estimated for the past 10 years.

TABLE 5. Comparison of Annual Population Oose Commitments for the Last 10 Years (person-rem)

$$
\underline{1975} \quad \underline{1976} \quad \underline{1977} \quad \underline{1978} \quad \underline{1979} \quad \underline{1980} \quad \underline{1981} \quad \underline{1982} \quad \underline{1983} \quad \underline{1984}
$$

$\begin{array}{lllllllllll}\text { Liquid } & 76 & 82 & 160 & 110 & 220 & 120 & 87 & 50 & 95 & 160\end{array}$

$\begin{array}{lllllllllll}\text { Air } & \frac{1300}{1300} & \frac{390}{470} & \frac{540}{700} & \frac{530}{640} & \frac{1600}{1800} & \frac{57}{180} & \frac{63}{150} & \frac{87}{140} & \frac{76}{170} & \frac{120}{280}\end{array}$

The reactor sites were compared as to the total population dose over the years of this study, 1975-1984. The sites were placed within six groupings depending on resulting population dose summed over each of the years through 1984:

$\begin{array}{lcc}\text { I } & \text { Greater than } 100 \text { person-rem } \\ \text { II } & 26-100 & " \\ \text { III } & 11-25 & " \\ \text { IV } & 2.6-10 & " \\ \text { V } & 1-2.5 & " \\ \text { VI } & \text { Less than } 1 & "\end{array}$

Table 6 shows the sites within the groups along with the reactor manufacturer, year of commercial operation commencement, and the indicated population doses in person-rem. The manufacturer codes are as follows:

AC AIlis Chatmers

B Babcox and Wilcox

CE Combustion Engineering

GE General Electric

W Westinghouse 


\section{TABLE 6. Total-Body Population Dosqs, from Nuclear Power PTant Effluents
During Normal Operations,
1975-1984}

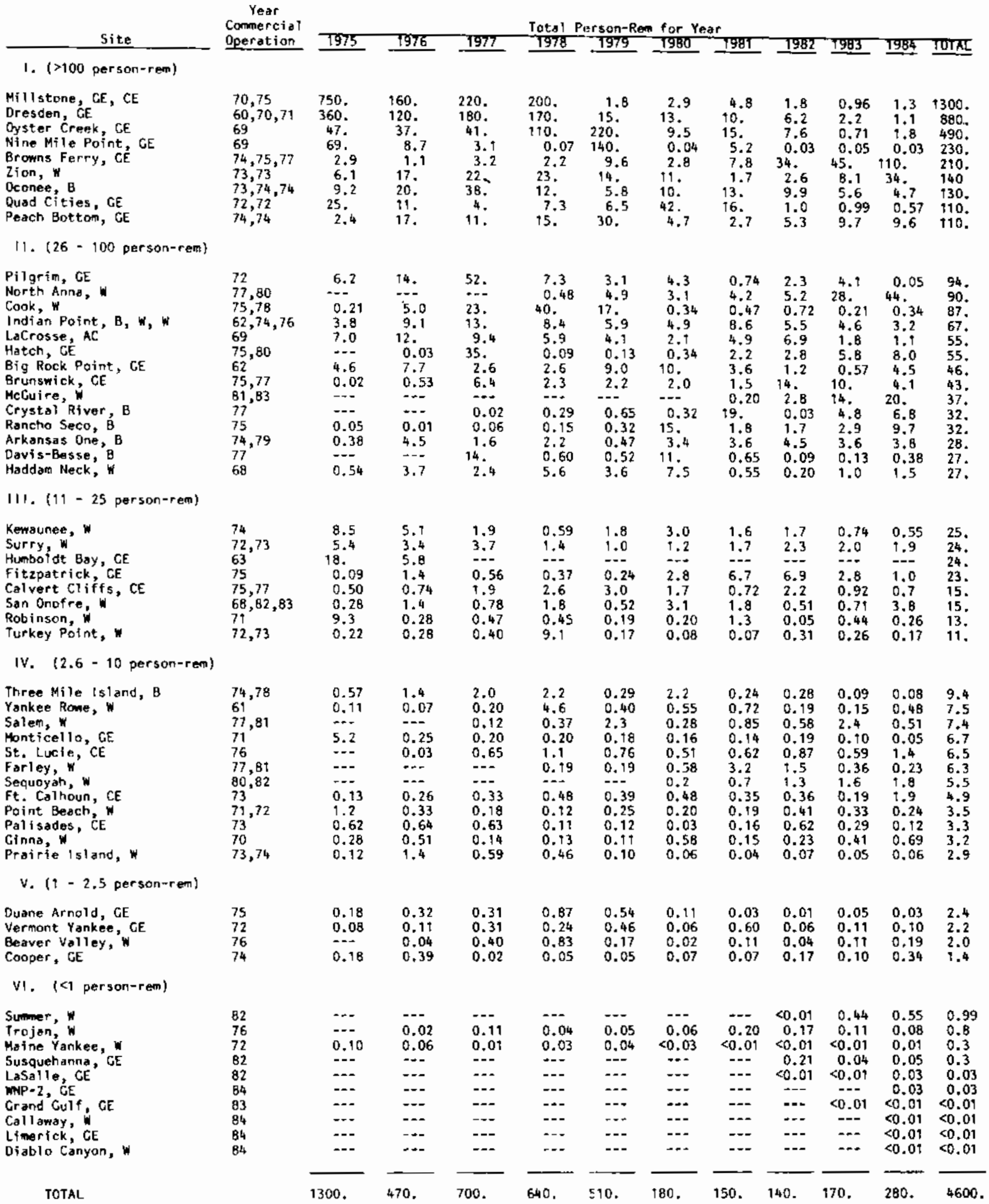

Note: Only the doses from the TMI accident, 1979, are excluded. 


\section{SITE SUMMARIES}

1984

2.1 
Site: ARKANSAS ONE

POPE COUNTY, ARKANSAS

Location: $\quad N 35.3100^{\circ}$ W $93.2308^{\circ}$

POPULATION DATA

Total Poputation Within 2-to-80-km Region: 1.9E5

Major Metropolitan Centers Within Region:

\begin{tabular}{lccc}
\multicolumn{1}{c}{ Center } & Population & \multicolumn{2}{c}{ Location } \\
Russeliville & 14,000 & $10 \mathrm{~km}$ & $\mathrm{E}$ \\
Conway & 20,000 & $76 \mathrm{~km}$ & ESE
\end{tabular}

SITE-SPECIFIC OATA - AIRBORNE PATHWAYS

Average Annuat State Production

of Crops and Animal Products

In $80-\mathrm{km}$ Radius Circle

Regional Productivity Factor:

Animat Grazing Factor:
Veg: $5.8 \mathrm{E} 6$ ki Togram

Milk: $4.8 E 7$ liter

Meat: $7.2 \mathrm{E} 7 \mathrm{ki}$ logram

1

0.7

Meteorology Period of Record: I JAN 75 - 3I OEC 75 Recovery: 97\%

SITE-SPECIFIC OATA - WATERBORNE PATHWAYS via ARKANSAS RIVER

Average River Flow

at Site: $36,000 \mathrm{ft}^{3} / \mathrm{s}$

Orinking Water:

Exposed Population: None

Fish:

Edible Harvest: $1.4^{(\mathrm{a})} \mathrm{kg} / \mathrm{yr}$

Dilution Factor: 1

(a) Average individuat consumption rates as given in the final Environmental Statement (FES) (1973) were used in Tieu of catch data. The reference to environmental statements here and on succeeding pages may be found in Table 3. 
POPULATION OOSE-COMMITMENT ESTIMATES ANO

AVERAGE INOIVIOUAL OOSE-COMMITMENT HISTOGRAM FOR

ARKANSAS ONE 1 AND 2

Dose Commitments (person-rem) from Waterborne Pathways

\begin{tabular}{|c|c|c|c|c|c|}
\hline & Tota 1 Body & GI -LLI & Thyroid & Bone & Liver \\
\hline $\begin{array}{l}\text { Infant } \\
\text { Child } \\
\text { Teen } \\
\text { Adult }\end{array}$ & $\begin{array}{l}0.0 \mathrm{E}+00 \\
1.5 \mathrm{E}-01 \\
3.0 \mathrm{E}-01 \\
3.2 \mathrm{E}+00\end{array}$ & $\begin{array}{l}0.0 \mathrm{E}+00 \\
2.0 \mathrm{E}-02 \\
4.0 \mathrm{E}-02 \\
3.0 \mathrm{E}-02\end{array}$ & $\begin{array}{l}0.0 \mathrm{E}+00 \\
2.1 \mathrm{E}-02 \\
1.5 \mathrm{E}-02 \\
1.0 \mathrm{E}-0 \mathrm{I}\end{array}$ & $\begin{array}{l}0.0 E+00 \\
8.1 E-01 \\
4.9 E-01 \\
2.8 E+00\end{array}$ & $\begin{array}{l}0.0 E+00 \\
9.0 E-01 \\
7.6 E-01 \\
4.5 E+00\end{array}$ \\
\hline OTAL & $3.7 E+00$ & $4.0 E-01$ & 1. $4 \mathrm{E}-01$ & $4.1 E+00$ & $6.2 \mathrm{E}+00$ \\
\hline
\end{tabular}

Dose Commitments (person-ren) from Airborne Pathways

\begin{tabular}{lcccccc} 
& Total Body & GI-LLI & Thyroid & Bone & Liver & Lung \\
\cline { 2 - 3 } Infant & $7.1 \mathrm{E}-04$ & $7.1 \mathrm{E}-04$ & $9.2 \mathrm{E}-04$ & $7.0 \mathrm{E}-04$ & $7.1 \mathrm{E}-04$ & $7.6 \mathrm{E}-04$ \\
Child & $8.0 \mathrm{E}-03$ & $7.9 \mathrm{E}-03$ & $9.2 \mathrm{E}-03$ & $7.8 \mathrm{E}-03$ & $7.9 \mathrm{E}-03$ & $8.7 \mathrm{E}-03$ \\
Teen & $5.8 \mathrm{E}-03$ & $5.8 \mathrm{E}-03$ & $6.3 \mathrm{E}-03$ & $5.7 \mathrm{E}-03$ & $5.8 \mathrm{E}-03$ & $6.8 \mathrm{E}-03$ \\
Adult & $3.5 \mathrm{E}-02$ & $3.5 \mathrm{E}-02$ & $3.7 \mathrm{E}-02$ & $3.4 \mathrm{E}-02$ & $3.5 \mathrm{E}-02$ & $3.8 \mathrm{E}-02$ \\
TOTAL & $5.0 \mathrm{E}-02$ & $4.9 \mathrm{E}-02$ & $5.3 \mathrm{E}-02$ & $4.9 \mathrm{E}-02$ & $4.9 \mathrm{E}-02$ & $5.4 \mathrm{E}-02$
\end{tabular}

Production/Consumption factors: (a)

$\begin{array}{rlrl}\text { Produce: } & <1 & \text { Milk: } 2.0 & \text { Meat: } 4.8 \\ \text { Fraction of Population Receiving an Average Individual } & \\ \text { Total-Body Dose Commitment from Airborne Pathways }\end{array}$

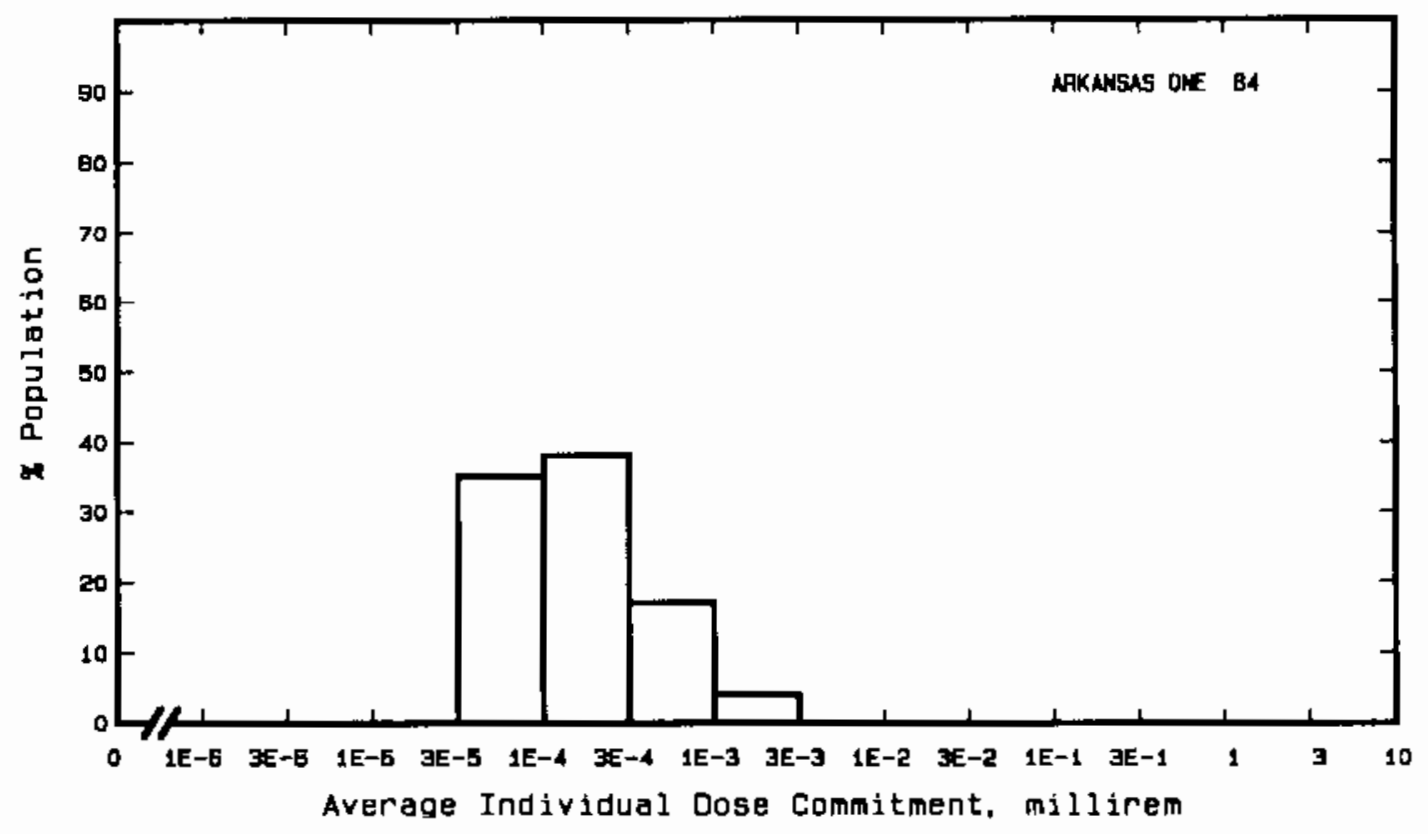

(a) See Appendix A, Page A-5, for explanation of this ratio. 
Site: BEAVER VALLEY

Location: $\quad$ N $40.6219^{\circ} \quad$ W $80.4339^{\circ}$

POPULATION OATA

Total Population Within 2-to-80-km Region: 3.6E6

Major Metropolitan Centers Within Region:

Center Poputation Location

Pittsburgh SMSA

Youngstown-Warren SMSA

Stuebenville-Weirton SMSA

Wheeling SMSA

New Castle
$2,300,000$

530,000

160,000

190,000

34,000
$42 \mathrm{~km} \quad \mathrm{ESE}$

$56 \mathrm{~km} \quad \mathrm{NNW}$

$33 \mathrm{~km} \quad \mathrm{SSW}$

$66 \mathrm{~km} \quad \mathrm{SSW}$

$43 \mathrm{~km} \quad \mathrm{~N}$

SITE-SPECIFIC DATA - AIRBORNE PATHWAYS

Average Annual State Production

of Crops and Animal Products

In $80-\mathrm{km}$ Radius Circle

Veg: $5.3 \mathrm{E} 7 \mathrm{kilogram}$

Milk: 5.3E8 Titer

Meat: $5.4 E 7$ kilogram

Regional Productivity Factor:

Animal Grazing Factor:

1

0.5

Meteorology Period of Record: I JAN 77 - 31 DEC 77 Recovery: 92\%

SITE-SPECIFIC DATA - WATERBORNE PATHWAYS via OHIO RIVER

Average River Flow

at Site: $30,000 \mathrm{ft}^{3} / \mathrm{s}$

Drinking Water:

Exposed Popstation: $(\$)^{200}$
Dilution Factor: 12

Fish:

Edible Harvest: $410 \mathrm{~kg} / \mathrm{yr}$ Dilution Factor: 1

(a) This factor accounts for the incomplete dilution of plant effluent by river at point of drinking water intake at Midland. 
POPULATION DOSE-COMMITMENT ESTIMATES AND

AVERAGE INDIVIDUAL DOSE-COMMITMENT HISTOGRAM FOR

BEAVER VALLEY

Dose Commitments (person-rem) from Waterborne Pathways

Total Body GI-LLI Thyroid Bone Liver

$\begin{array}{llllll}\text { Infant } & 8.9 \mathrm{E}-04 & 8.9 \mathrm{E}-04 & 1.0 \mathrm{E}-03 & 5.9 \mathrm{E}-05 & 9.4 \mathrm{E}-04 \\ \text { Child } & 1.0 \mathrm{E}-02 & 1.0 \mathrm{E}-02 & 1.1 \mathrm{E}-02 & 6.5 \mathrm{E}-04 & 1.0 \mathrm{E}-02 \\ \text { Teen } & 3.8 \mathrm{E}-03 & 4.1 \mathrm{E}-03 & 4.0 \mathrm{E}-03 & 1.7 \mathrm{E}-04 & 4.0 \mathrm{E}-03 \\ \text { AduIt } & 3.3 \mathrm{E}-02 & 3.5 \mathrm{E}-02 & 3.4 \mathrm{E}-02 & 1.1 \mathrm{E}-03 & 3.3 \mathrm{E}-02 \\ \text { TOTAL } & 4.8 \mathrm{E}-02 & 5.0 \mathrm{E}-02 & 5.0 \mathrm{E}-02 & 1.9 \mathrm{E}-03 & 4.9 \mathrm{E}-02\end{array}$

Dose Conmitments (person-rem) from Airborne Pathways

$$
\text { Total Body GI-LLI Thyroid Bone Liver Lung }
$$

$\begin{array}{lllllll}\text { Infant } & 2.1 \mathrm{E}-03 & 2.0 \mathrm{E}-03 & 1.3 \mathrm{E}-02 & 1.7 \mathrm{E}-03 & 2.1 \mathrm{E}-03 & 2.2 \mathrm{E}-03 \\ \text { Child } & 2.3 \mathrm{E}-02 & 2.3 \mathrm{E}-02 & 8.3 \mathrm{E}-02 & 1.9 \mathrm{E}-02 & 2.3 \mathrm{E}-02 & 2.6 \mathrm{E}-02\end{array}$

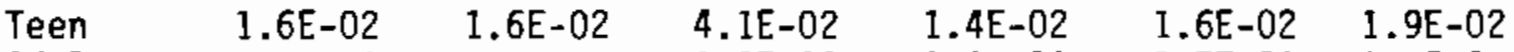

Adult $9.6 \mathrm{E}-02 \quad 9.5 \mathrm{E}-02 \quad 1.8 \mathrm{E}-01 \quad 8.2 \mathrm{E}-02 \quad 9.5 \mathrm{E}-02 \quad 1.1 \mathrm{E}-01$

TOTAL $\quad 1.4 \mathrm{E}-01 \quad 1.4 \mathrm{E}-01 \quad 3.2 \mathrm{E}+01 \quad 1.2 \mathrm{E}-01 \quad 1.4 \mathrm{E}-01 \quad 1.5 \mathrm{E}-01$

Production/Consumption factors:

Produce: <1 Milk: $1.1 \quad$ Meat: <I

Fraction of Population Receiving an Average Individual

Total-Body Dose Commitment from Airborne Pathways

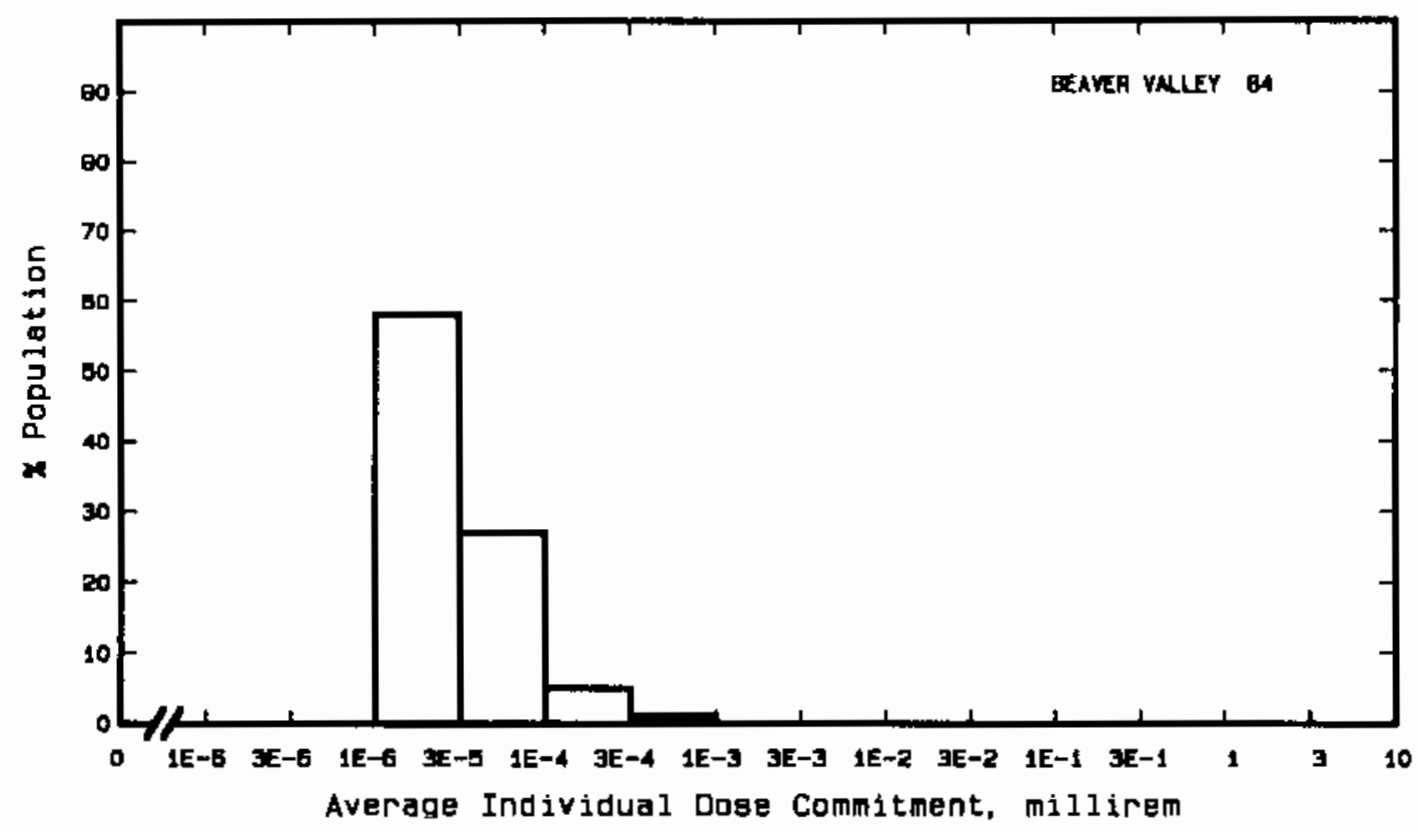


Site: BIG ROCK POINT

CHARLEVOIX COUNTY, MICHIGAN
Location:
N $45.3592^{\circ}$
W $85.1947^{\circ}$

POPULATION DATA

Total Population Within 2-to-80-km Region: 1.6E5

Major Metropolitan Centers Within Region:

\begin{tabular}{|c|c|c|}
\hline Center & Population & Location \\
\hline $\begin{array}{l}\text { Traverse City } \\
\text { Petoskey } \\
\text { Cheboygan }\end{array}$ & $\begin{array}{r}16,000 \\
6,100 \\
5,100\end{array}$ & $\begin{array}{lll}75 \mathrm{~km} & \text { SSW } \\
18 \mathrm{~km} & \mathrm{E} \\
65 \mathrm{~km} & \text { ENE }\end{array}$ \\
\hline
\end{tabular}

SITE-SPECIFIC DATA - AIRBORNE PATHWAYS

Average Annual State Production

Of Crops and Animal Products

In $80-\mathrm{km}$ Radius Circle

Regional Productivity Factor:

Animal Grazing Factor:
Veg: $6.9 \mathrm{E} 7 \mathrm{kilogram}$

Milk: $2.9 \mathrm{E} 8$ Titer

Meat: $4.5 E 7$ kilogram

0.5

0.5

Meteorology Period of Record: 9 FEB 6I - 8 FEB 63 Recovery: 85\%

SITE-SPECIFIC DATA - WATERBORNE PATHWAYS via LAKE MICHIGAN

Average Dilution Flow

from Plant: $110 \mathrm{ft}^{3} / \mathrm{s}$

Drinking Water:

Exposed Population: $30, \rho \mathrm{BP}$ (a)

Dilution Factor: $4.9 \mathrm{E}-5$

Fish:

Edible Harvest: (c) $\mathrm{kg} / \mathrm{yr}$

Dilution Factor: 0.01

(a) Population exposed to contaminated drinking water derived from information obtained from J. Hennigan, Division of Radiation Health, Bureau of Environment and Health, Michigan Department of Pubtic Health.

(b) Drinking water dilution factor estimated by averaging dilution factor derived from Figure 6B-5, Vol. 1 of WASH-1258 (1973) suitably weighted for population.

(c) Generic consumption rate used (Table A-1). 
POPULATION DOSE-COMMITMENT ESTIMATES AND

AVERAGE INDIVIDUAL DOSE-COMMITMENT HISTOGRAM FOR

BIG ROCK POINT

Dose Comitments (person-rem) from Waterborne Pathways

Total Body GI-LLI Thyroid Bone Liver

$\begin{array}{llllll}\text { Infant } & 1.4 \mathrm{E}-07 & 4.5 \mathrm{E}-08 & 1.6 \mathrm{E}-05 & 7.9 \mathrm{E}-07 & 8.1 \mathrm{E}-07 \\ \text { Child } & 2.0 \mathrm{E}-02 & 2.1 \mathrm{E}-03 & 9.0 \mathrm{E}-03 & 1.1 \mathrm{E}-01 & 1.2 \mathrm{E}-01 \\ \text { Teen } & 3.9 \mathrm{E}-02 & 4.4 \mathrm{E}-03 & 6.5 \mathrm{E}-03 & 6.6 \mathrm{E}-02 & 1.0 \mathrm{E}-01 \\ \text { Adult } & 4.2 \mathrm{E}-01 & 3.8 \mathrm{E}-02 & 4.3 \mathrm{E}-02 & 3.8 \mathrm{E}-01 & 6.0 \mathrm{E}-01 \\ \text { TOTAL } & 4.8 \mathrm{E}-01 & 4.5 \mathrm{E}-02 & 5.8 \mathrm{E}-02 & 5.5 \mathrm{E}-01 & 8.2 \mathrm{E}-01\end{array}$

Dose Commitments (person-rem) from Airborne Pathways

Total Body GI-LLI Thyroid Bone Liver Lung

Infant

Child

$5.7 \mathrm{E}-02$

$5.7 \mathrm{E}-02$

$6.3 \mathrm{E}-02$

$5.7 \mathrm{E}-02$

5.7E-02

$6.4 \mathrm{E}-01$

$6.4 \mathrm{E}-01$

7.0E-0I

$6.4 \mathrm{E}-01$

$6.4 \mathrm{E}-01$

4.7E-01

4.7E-0I

4. $9 \mathrm{E}-01$

4.7E-01

4.7E-01

5. $9 \mathrm{E}-02$

Adult

$2.8 \mathrm{E}+00$

$2.8 \mathrm{E}+00$

2. $9 \mathrm{E}+00$

$2.8 \mathrm{E}+00$

$2.8 \mathrm{E}+00$

6. $6 \mathrm{E}-0 \mathrm{I}$

TOTAL

$4.0 \mathrm{E}+00$

$4.0 \mathrm{E}+00$

4. $2 \mathrm{E}+00$

4. $0 \mathrm{E}+00$

4. $0 \mathrm{E}+00$

4.9E-0I

2. $9 E+00$

4. $1 \mathrm{E}+00$

Production/Consumption factors:

Produce: 1.0

Milk: 6.6

Meat: 1.7

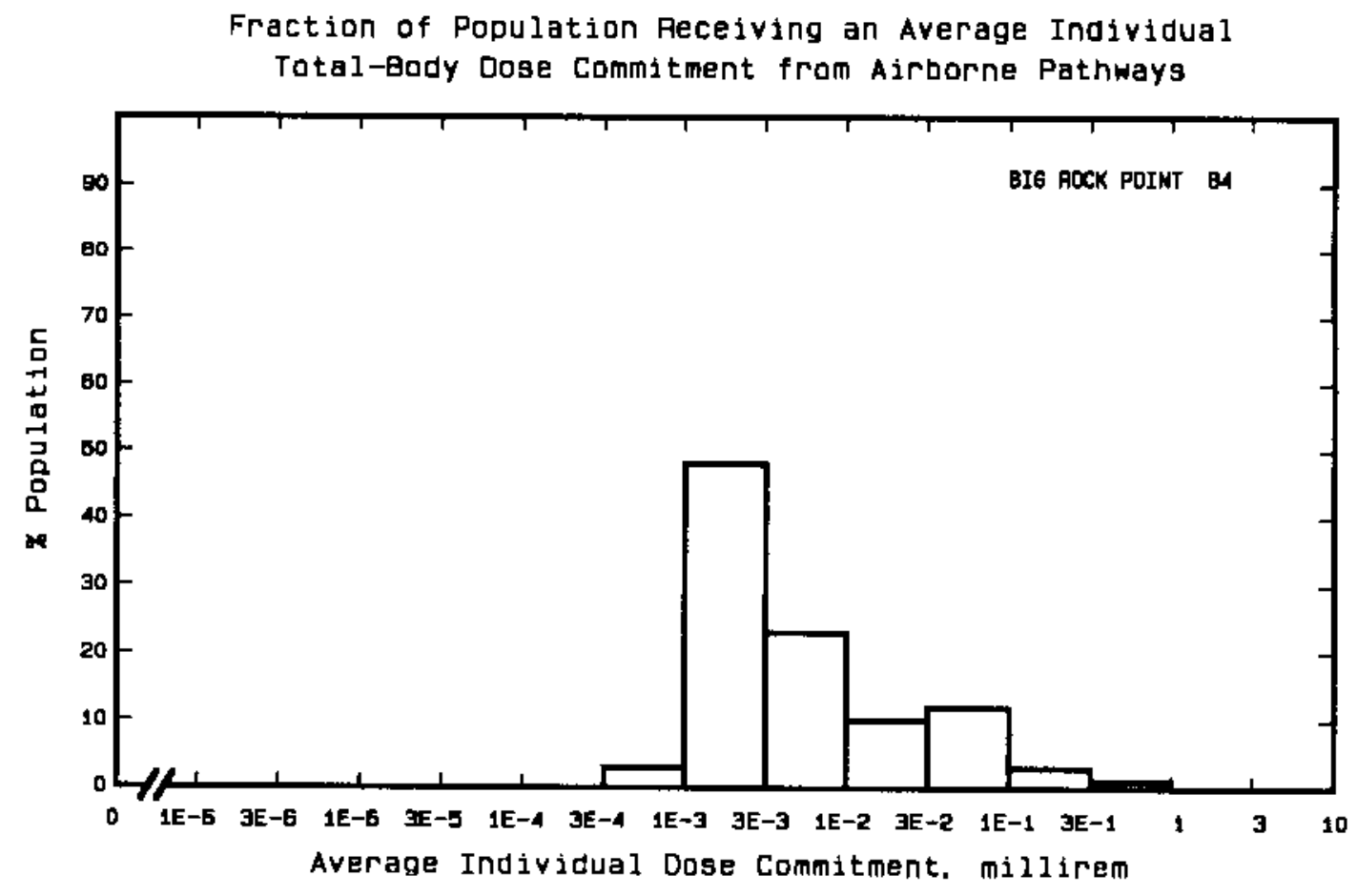


Site: BROWNS FERRY

OECATUR, ALABAMA

Location: $\quad \mathrm{N} 34.7042^{\circ} \quad$ W $87.1186^{\circ}$

POPULATION OATA

Total Population Within 2-to-80-km Region: 7.3E5

Major Metropolitan Centers Within Region:

$\underline{\text { Center }}$

Huntsville SMSA

Florence SMSA

Decatur

Athens

Cullman
Population

310,000

140,000

42,000

15,000

13,000
Location

$49 \mathrm{~km} \quad \mathrm{E}$

$52 \mathrm{~km}$ WNW

$16 \mathrm{~km} \quad \mathrm{SE}$

$17 \mathrm{~km} \quad \mathrm{NE}$

$64 \mathrm{~km}$ SSE

SITE-SPECIFIC DATA - AIRBORNE PATHWAYS

Average Annual State Production

of Crops and Animal Products

In 80-km Radius Circle

Regional Productivity Factor:

Animal Grazing Factor:
Veg: $1.7 \mathrm{E} 7 \mathrm{kilogram}$

Milk: $5.7 \mathrm{E} 7$ Titer

Meat: 8.6E7 kilogram

1

0.7

Meteorology Period of Record: 1 JAN 74 - 31 DEC 75 Recovery: 94\%

SITE-SPECIFIC DATA - WATERBORNE PATHWAYS via TENNESSEE RIVER at WHEELER LAKE

Average River Flow

at Site: $45,000 \mathrm{ft}^{3} / \mathrm{s}$

Orinking Water:

Exposed Population: 25,000

DiTution Factor: 1

Fish:

Edible Harvest: $1.6 \mathrm{E} 6 \mathrm{~kg} / \mathrm{yr}$

Dilution Factor: 1 
POPULATION OOSE-COMMITMENT ESTIMATES ANO

AVERAGE INDIVIDUAL DOSE-COMMITMENT HISTOGRAM FOR

BROWNS FERRY 1,2 ANO 3

Oose Commitments (person-rem) from Waterborne Pathways

Total Body GI-LLI Thyroid Bone Liver

$\begin{array}{llllll}\text { Infant } & 2.7 \mathrm{E}-04 & \mathrm{I}-\mathrm{IE}-04 & 3.2 \mathrm{E}-03 & 1.7 \mathrm{E}-03 & 2.4 \mathrm{E}-03 \\ \text { Child } & 7.7 \mathrm{E}-01 & 5.0 \mathrm{E}-02 & 3.7 \mathrm{E}-02 & 3.2 \mathrm{E}+00 & 3.9 \mathrm{E}+00 \\ \text { Teen } & 1.4 \mathrm{E}+00 & 1.0 \mathrm{E}-01 & 1.8 \mathrm{E}-02 & 1.9 \mathrm{E}+00 & 3.4 \mathrm{E}+00 \\ \text { Adult } & 1.5 \mathrm{E}+01 & 9.2 \mathrm{E}-01 & 1.2 \mathrm{E}-0 \mathrm{I} & 1.1 \mathrm{E}+0 \mathrm{I} & 2.0 \mathrm{E}+0 \mathrm{I} \\ \text { TOTAL } & 1.7 \mathrm{E}+01 & 1.1 \mathrm{E}+00 & 1.8 \mathrm{E}-01 & 1.6 \mathrm{E}+01 & 2.7 \mathrm{E}+01\end{array}$

Dose Commitments (person-rem) from Airborne Pathways

\begin{tabular}{lcccccc} 
& Total Body & GI-LLI & Thyroid & Bone & Liver & Lung \\
\cline { 2 - 3 } Infant & $1.3 \mathrm{E}+00$ & $1.3 \mathrm{E}+00$ & $1.3 \mathrm{E}+00$ & $1.3 \mathrm{E}+00$ & $1.3 \mathrm{E}+00$ & $1.3 \mathrm{E}+00$ \\
ChiTd & $1.4 \mathrm{E}+01$ & $1.4 \mathrm{E}+01$ & $1.5 \mathrm{E}+01$ & $1.4 \mathrm{E}+01$ & $1.4 \mathrm{E}+01$ & $1.5 \mathrm{E}+01$ \\
Teen & $1.0 \mathrm{E}+01$ & $1.0 \mathrm{E}+01$ & $1.1 \mathrm{E}+01$ & $1.0 \mathrm{E}+01$ & $1.0 \mathrm{E}+01$ & $1.1 \mathrm{E}+01$ \\
Adult & $6.3 \mathrm{E}+01$ & $6.3 \mathrm{E}+01$ & $6.3 \mathrm{E}+01$ & $6.3 \mathrm{E}+01$ & $6.3 \mathrm{E}+01$ & $6.5 \mathrm{E}+01$ \\
TOTAL & $8.9 \mathrm{E}+01$ & $8.9 \mathrm{E}+01$ & $9.0 \mathrm{E}+01$ & $8.9 \mathrm{E}+01$ & $8.9 \mathrm{E}+01$ & $9.2 \mathrm{E}+01$
\end{tabular}

Production/Consumption factors:

Produce: $<1$ Milk: $<1 \quad$ Meat: 1.5

Fraction of Population Receiving an Average Individual

Total-Body Dose Commitment from Airborne Pathways

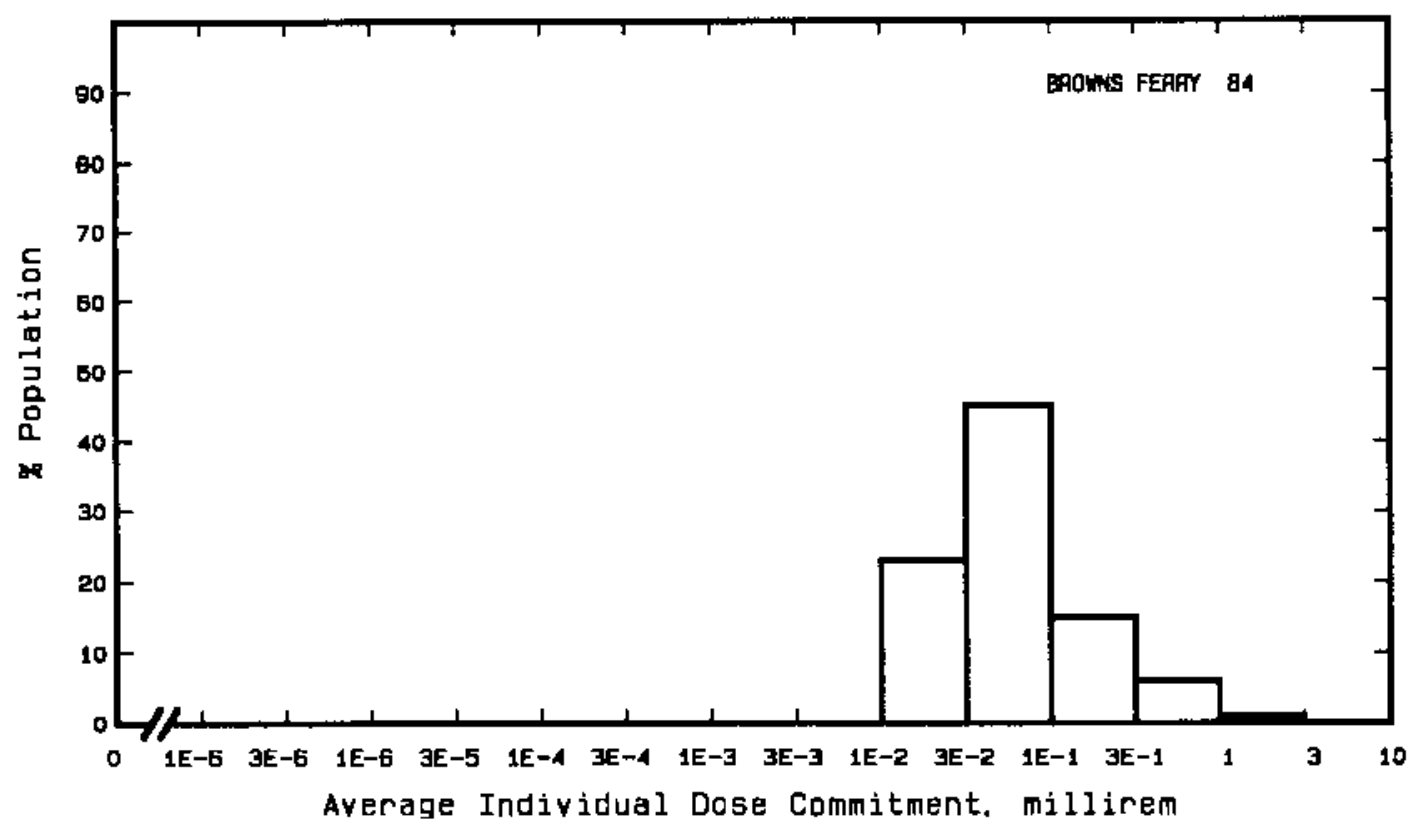


Site: BRUNSWICK

BRUNSWICK COUNTY, NORTH CAROLINA

Location: $\quad$ N $33.9583^{\circ}$ W $78.0106^{\circ}$

POPULATION DATA

Total Population Within 2-to-80-km Region: 2.2E5

Major Metropolitan Centers Within Region:

Center Population Location

Wilmington SMSA $\quad 140$, D00 $32 \mathrm{~km}$ NNE

Whiteville $\quad 5,600 \quad 75 \mathrm{~km}$ WNW

N. Myrtle Beach 4,000 $65 \mathrm{~km}$ WSW

SITE-SPECIFIC DATA - AIRBORNE PATHWAYS

Average Annual State Production

of Crops and Animal Products

In 80-km Radius Circle

Regional Productivity Factor:

Animal Grazing Factor:

Meteorology Period of Record: 1 JAN 75 - 31 DEC 75 Recovery: 93\%
Veg: $2.6 \mathrm{E} 7 \mathrm{kilogram}$

Milk: 1.0E8 liter

Meat: $5.8 E 7$ kilogram

0.3

0.7

SITE-SPECIFIC DATA - WATERBORNE PATHWAYS via ATLANTIC OCEAN

Average Dilution Flow

from Plant: $3,100 \mathrm{ft}^{3} / \mathrm{s}$

Fish:

Edible Harvest: $2.1 \mathrm{E5}{ }^{(\mathrm{a})} \mathrm{kg} / \mathrm{yr}$

Oilution Factor: 0.001

Invertebrates:

Edible Harvest: $1.1 \mathrm{E5}{ }^{(\mathrm{a})} \mathrm{kg} / \mathrm{yr}$

Dilution Factor: 0.002

(a) Fish and invertebrate harvests together total harvest given in FES (1973). 
POPULATION DOSE-COMMITMENT ESTIMATES AND

AVERAGE INDIVIDUAL DOSE-COMMITMENT HISTOGRAM FOR

BRUNSWICK 1 AND 2

Oose Commitments (person-rem) from Waterborne Pathways

Total Body GI-LLI Thyroid Bone Liver

$\begin{array}{llllll}\text { Infant } & 0.0 \mathrm{E}+00 & 0.0 \mathrm{E}+00 & 0.0 \mathrm{E}+00 & 0.0 \mathrm{E}+00 & 0.0 \mathrm{E}+00 \\ \text { Child } & 2.6 \mathrm{E}-05 & 4.3 \mathrm{E}-05 & 3.3 \mathrm{E}-06 & 3.7 \mathrm{E}-05 & 3.5 \mathrm{E}-05 \\ \text { Teen } & 1.8 \mathrm{E}-05 & 8.8 \mathrm{E}-05 & 2.3 \mathrm{E}-06 & 2.1 \mathrm{E}-05 & 2.8 \mathrm{E}-05 \\ \text { Aduit } & 1.2 \mathrm{E}-05 & 7.7 \mathrm{E}-04 & 1.5 \mathrm{E}-05 & 1.3 \mathrm{E}-04 & 1.7 \mathrm{E}-04 \\ \text { T0TAL } & 1.6 \mathrm{E}-04 & 9.0 \mathrm{E}-04 & 2.1 \mathrm{E}-05 & 1.8 \mathrm{E}-04 & 2.3 \mathrm{E}-04\end{array}$

Oose Commitments (person-rem) from Airborne Pathways

Total Body GI-LLI Thyroid Bone Liver Lung

$\begin{array}{lllllll}\text { Infant } & 5.9 \mathrm{E}-02 & 5.9 \mathrm{E}-02 & 8.3 \mathrm{E}-02 & 5.9 \mathrm{E}-02 & 5.9 \mathrm{E}-02 & 6.0 \mathrm{E}-02 \\ \text { Child } & 6.5 \mathrm{E}-01 & 6.5 \mathrm{E}-01 & 8.1 \mathrm{E}-01 & 6.6 \mathrm{E}-01 & 6.6 \mathrm{E}-01 & 6.7 \mathrm{E}-01 \\ \text { Teen } & 4.8 \mathrm{E}-01 & 4.8 \mathrm{E}-01 & 5.5 \mathrm{E}-01 & 4.8 \mathrm{E}-01 & 4.8 \mathrm{E}-01 & 5.3 \mathrm{E}-01 \\ \text { Adu1t } & 2.9 \mathrm{E}+00 & 2.9 \mathrm{E}+00 & 3.2 \mathrm{E}+00 & 2.9 \mathrm{E}+00 & 2.9 \mathrm{E}+00 & 3.0 \mathrm{E}+00 \\ \text { TOTAL } & 4.1 \mathrm{E}+00 & 4.1 \mathrm{E}+00 & 4.6 \mathrm{E}+00 & 4.1 \mathrm{E}+00 & 4.1 \mathrm{E}+00 & 4.2 \mathrm{E}+00\end{array}$

Production/Consumption factors:

Produce: <1 Milk: 1.1 Meat: 1.0

Fraction of Population Receiving an Average Individual

Total-Body Dose Commitment from Airborne Pathways

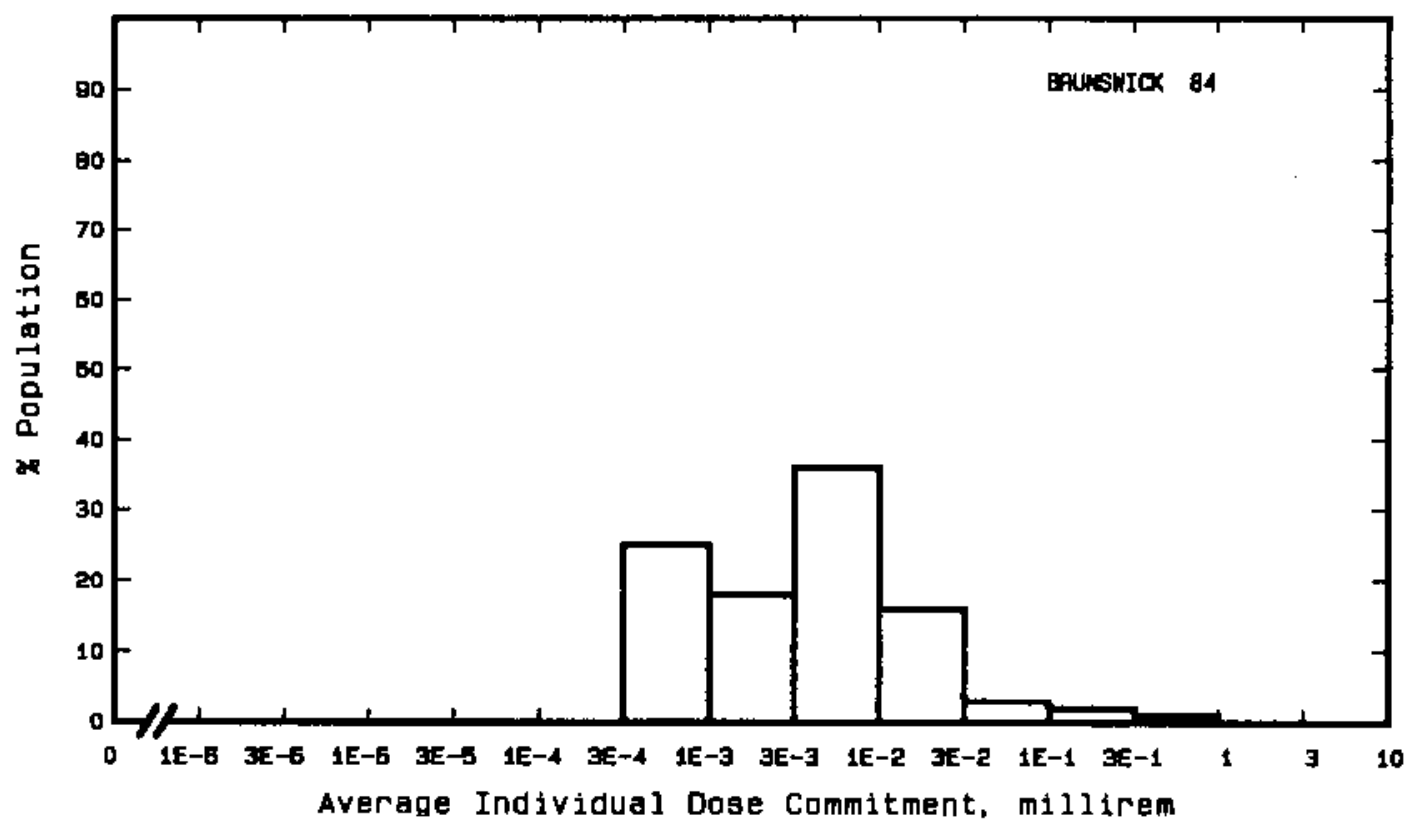


Site: CALLAWAY

FULTON, MISSOURI

Location: $\quad N 38.7618^{\circ} \quad$ W $91.7979^{\circ}$

POPULATION DATA

Tota 7 Population Within 2-to-80-km Region: 3.6E5

Major Metropolitan Centers Within Region:

\begin{tabular}{|c|c|c|c|}
\hline Center & Population & \multicolumn{2}{|c|}{ Location } \\
\hline Columbia & 59,000 & $48 \mathrm{~km}$ & WNW \\
\hline Jefferson City & 33,000 & $40 \mathrm{~km}$ & WSW \\
\hline Mexico & 12,000 & $45 \mathrm{~km}$ & NNW \\
\hline Washington & 8,500 & $69 \mathrm{~km}$ & ESE \\
\hline Fulton & 13,000 & $19 \mathrm{~km}$ & $\mathrm{NW}$ \\
\hline
\end{tabular}

SITE-SPECIFIC DATA - AIRBORNE PATHWAYS

Average Annual State Production

of Crops and Animal Products

In 80-km Radius Circle

Veg: $3.3 E 7$ ki logram

Milk: $1.5 \mathrm{E} 8$ liter

Regional Productivity Factor:

Animal Grazing Factor:

Meat: $1.9 E 8$ kiTogram

Meteorology Period of Record: 4 MAY $74-3$ MAY 75 Recovery: 89\%

SITE-SPECIFIC DATA - WATERBORNE PATHWAYS via MISSOURI RIVER

Average River Flow

at Site: $80,000 \mathrm{ft}^{3} / \mathrm{s}$

Drinking Water:

Exposed Poputation: None

Fish:

Edible Harvest: $1.0 \mathrm{E} 3 \mathrm{~kg} / \mathrm{yr}$

Dilution Factor: I 
POPULATION DOSE-COMMITMENT ESTIMATES AND
AVERAGE INDIVIDUAL DOSE-COMMITMENT HISTOGRAM FOR

CALLAWAY

Dose Commitments (person-rem) from Waterborne Pathways

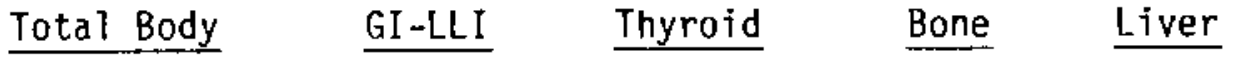

$\begin{array}{llllll}\text { Infant } & 0.0 \mathrm{E}+00 & 0.0 \mathrm{E}+00 & 0.0 \mathrm{E}+00 & 0.0 \mathrm{E}+00 & 0.0 \mathrm{E}+00 \\ \text { Child } & 4.5 \mathrm{E}-09 & 4.6 \mathrm{E}-09 & 4.7 \mathrm{E}-09 & 3.4 \mathrm{E}-10 & 4.7 \mathrm{E}-09 \\ \text { Teen } & 4.1 \mathrm{E}-09 & 4.3 \mathrm{E}-09 & 4.2 \mathrm{E}-09 & 2.0 \mathrm{E}-10 & 4.2 \mathrm{E}-09 \\ \text { Adult } & 3.2 \mathrm{E}-08 & 3.5 \mathrm{E}-08 & 3.3 \mathrm{E}-08 & 1.1 \mathrm{E}-09 & 3.3 \mathrm{E}-08 \\ \text { TOTAL } & 4.1 \mathrm{E}-08 & 4.4 \mathrm{E}-08 & 4.2 \mathrm{E}-08 & 1.7 \mathrm{E}-09 & 4.2 \mathrm{E}-08\end{array}$

Dose Commitments (person-rem) from Airborne Pathways Total Body GI-LLI Thyroid Bone Liver Lung

$\begin{array}{lllllll}\text { Infant } & 2.1 \mathrm{E}-05 & 2.1 \mathrm{E}-05 & 2.1 \mathrm{E}-05 & 1.9 \mathrm{E}-05 & 2.1 \mathrm{E}-05 & 2.3 \mathrm{E}-05 \\ \text { Child } & 2.4 \mathrm{E}-04 & 2.4 \mathrm{E}-04 & 2.5 \mathrm{E}-04 & 2.2 \mathrm{E}-04 & 2.4 \mathrm{E}-04 & 2.7 \mathrm{E}-04 \\ \text { Teen } & 1.8 \mathrm{E}-04 & 1.8 \mathrm{E}-04 & 1.8 \mathrm{E}-04 & 1.6 \mathrm{E}-04 & 1.8 \mathrm{E}-04 & 2.0 \mathrm{E}-04 \\ \text { Adult } & 1.1 \mathrm{E}-03 & 1.1 \mathrm{E}-03 & 1.1 \mathrm{E}-03 & 9.6 \mathrm{E}-04 & 1.1 \mathrm{E}-03 & 1.1 \mathrm{E}-03 \\ \text { TOTAL } & 1.5 \mathrm{E}-03 & 1.5 \mathrm{E}-03 & 1.5 \mathrm{E}-03 & 1.4 \mathrm{E}-03 & 1.5 \mathrm{E}-03 & 1.6 \mathrm{E}-03\end{array}$

Production/Consumption factors:

Produce: <1 Milk: 2.9 Meat: 6.0

Fraction of Population Receiving an Average Individual Total-Body Dose Commitment from Airborne Pathways

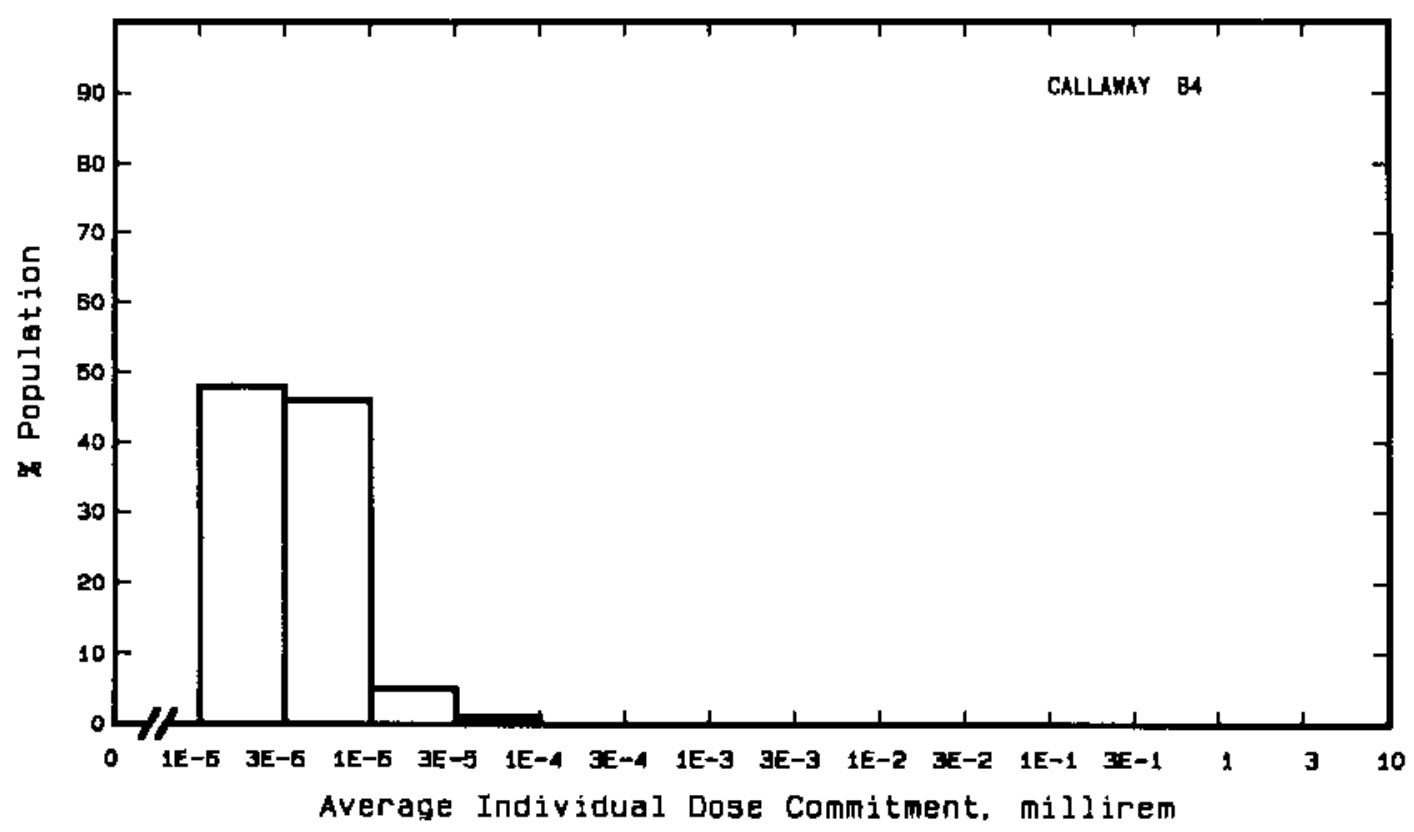


Site: CALVERT CLIFFS

LUSBY, MARYLAND

Location: N $38.4347^{\circ}$ W $76.4419^{\circ}$

POPULATION DATA

Total Population Within 2-to-80-km Region: 2.7E6

Major Metropolitan Centers Within Region:

\begin{tabular}{lrrrr}
\multicolumn{1}{c}{ Center } & Population & \multicolumn{2}{c}{ Location } \\
\cline { 2 - 2 } & & & \\
Washington, DC SMSA $(3 / 4)$ & $3,100,000$ & & $73 \mathrm{~km}$ & NW \\
Bowie & 34,000 & $71 \mathrm{~km}$ & NNW \\
Annapolis & 32,000 & $61 \mathrm{~km}$ & $\mathrm{~N}$ \\
Waldorf & 9,800 & $46 \mathrm{~km}$ & WNW \\
Salisbury & 16,000 & $75 \mathrm{~km}$ & $\mathrm{E}$ \\
Cambridge & 12,000 & $32 \mathrm{~km}$ & ENE
\end{tabular}

SITE-SPECIFIC DATA - AIRBORNE PATHWAYS

Average Annual State Production

of Crops and Anima1 Products

In 80-km Radius Circle

Regiona1 Productivity Factor:

Animal Grazing Factor:

Meteorology Period of Record: I JAN 75 - 3l DEC 75 Recovery: 96\%

Veg: $4.5 E 7$ kilogram

Milk: 5.0 E8 liter

Meat: $6.2 E 7$ kilogram

0.6

0.6

SITE-SPECIFIC DATA - WATERBORNE PATHWAYS via CHESAPEAKE BAY

Average Dilution Flow

from PTant: $2,500 \mathrm{ft}^{3} / \mathrm{s}$

Fish:

Edible Harvest: $1.0 \mathrm{E} 7 \mathrm{~kg} / \mathrm{yr}$
Dilution Factor: 0.062

Invertebrates:

Edible Harvest: $7.4 \mathrm{E} 6 \mathrm{~kg} / \mathrm{yr}$

Dilution Factor: $0.062^{(a)}$

(a) Dilutions given in FES (1973). 
POPULATION DOSE-COMMITMENT ESTIMATES AND

AVERAGE INOIVIDUAL DOSE-COMMITMENT HISTOGRAM FOR

CALVERT CLIFFS 1 AND 2

Oose Commitments (person-rem) from Waterborne Pathways

Total Body GI-LLI Thyroid Bone Liver

Infant

Child

Teen

Adu $1 \mathrm{t}$

$$
0.0 \mathrm{E}+00
$$

$0.0 \mathrm{E}+00$

$0 . D E+00$

$0.0 \mathrm{E}+00$

$0.0 \mathrm{E}+00$

$4.8 \mathrm{E}-02$

7. $0 \mathrm{E}-01$

$1.1 \mathrm{E}-01$

9. $3 \mathrm{E}-02$

1. 1E-DI

5. $3 \mathrm{E}-02$

1. $5 \mathrm{E}+00$

7. $6 \mathrm{E}-02$

5.6E-02

9. $2 \mathrm{E}-02$

4.7E-01

1. $3 \mathrm{E}+01$

5. $1 \mathrm{E}-01$

3. 3E-01

5.5E-01

TOTAL

$5.7 \mathrm{E}-01$

1. $5 \mathrm{E}+01$

$6.9 \mathrm{E}-01$

4.8E-01

$7.5 E-01$

Dose Comtitments (person-rem) from Airborne Pathways

$$
\text { Total Body GI-LLI Thyroid Bone Liver Lung }
$$

$\begin{array}{lllllll}\text { Infant } & 2.0 \mathrm{E}-03 & 1.9 \mathrm{E}-03 & 7.9 \mathrm{E}-\mathrm{D} 2 & 2.1 \mathrm{E}-03 & 2.1 \mathrm{E}-03 & 2.0 \mathrm{E}-03 \\ \text { Child } & 2.2 \mathrm{E}-02 & 2.1 \mathrm{E}-02 & 4.3 \mathrm{E}-01 & 2.2 \mathrm{E}-02 & 2.2 \mathrm{E}-02 & 2.3 \mathrm{E}-02 \\ \text { Teen } & 1.5 \mathrm{E}-02 & 1.5 \mathrm{E}-02 & 1.8 \mathrm{E}-01 & 1.5 \mathrm{E}-02 & 1.6 \mathrm{E}-02 & 1.8 \mathrm{E}-02 \\ \text { Adu1t } & 9.3 \mathrm{E}-02 & 9.2 \mathrm{E}-02 & 6.1 \mathrm{E}-01 & 9.2 \mathrm{E}-02 & 9.3 \mathrm{E}-02 & 1.0 \mathrm{E}-01 \\ \text { TOTAL } & 1.3 \mathrm{E}-01 & 1.3 \mathrm{E}-01 & 1.3 \mathrm{E}+00 & 1.3 \mathrm{E}-01 & 1.3 \mathrm{E}-01 & 1.4 \mathrm{E}-01\end{array}$

Production/Consumption factors:

Produce: $<1 \quad$ Milk: $<1 \quad$ Meat: <1

Fraction of Population Receiving an Average Individual

Total-Body Dose Commitment from Airborne Pethways

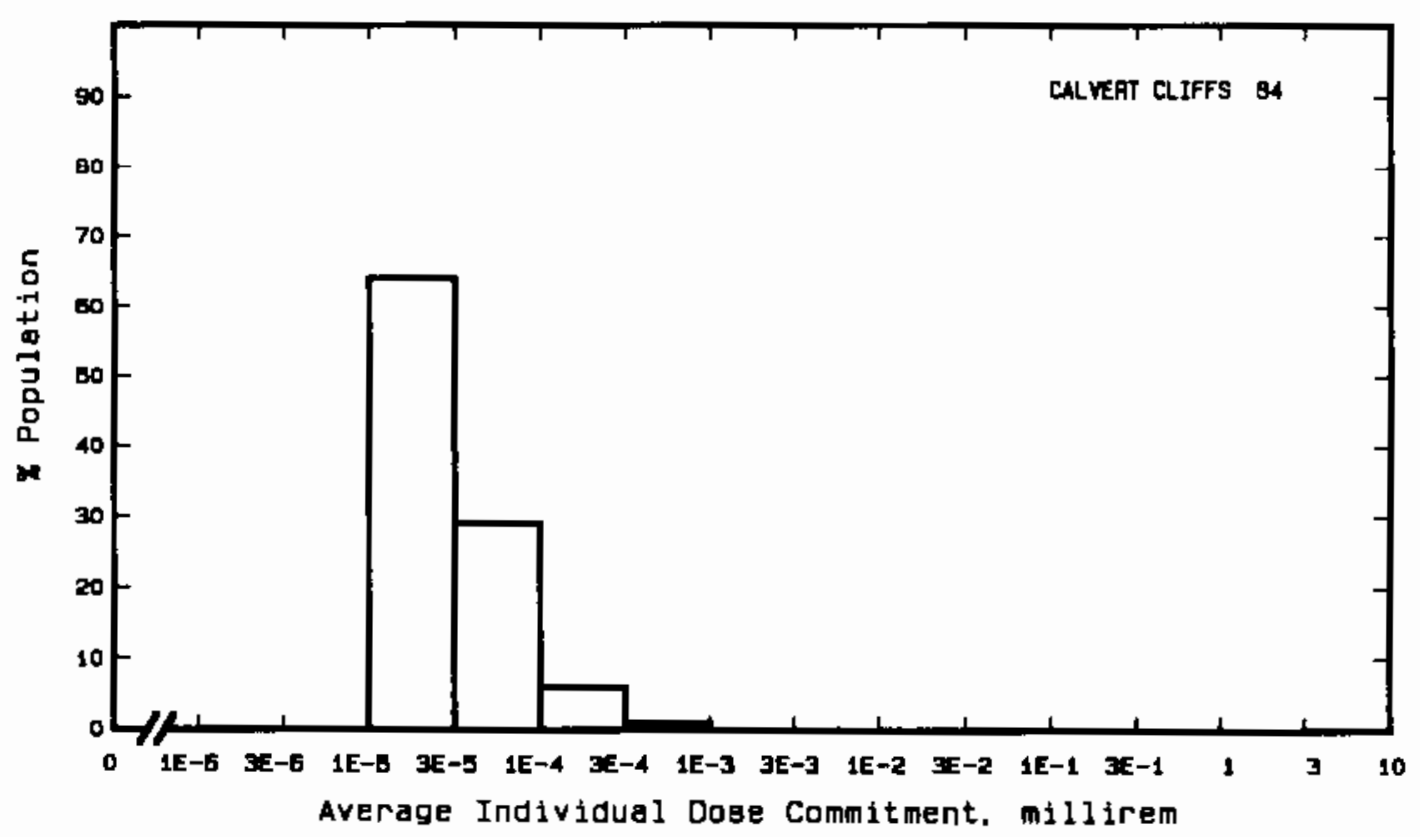


Site: $\operatorname{COOK}$

BENTON HARBOR, MICHIGAN

Location: $\quad \mathrm{N} 41.9761^{\circ} \quad$ W $B 6.5664^{\circ}$

POPULATION DATA

Total Population Within 2-to-80-km Region: 1.156

Major Metropolitan Centers Within Region:

\begin{tabular}{|c|c|c|c|}
\hline Center & Population & \multicolumn{2}{|c|}{ Location } \\
\hline ary & 150,000 & $77 \mathrm{~km}$ & \\
\hline outh Bend SMSA & 280,000 & $42 \mathrm{~km}$ & $\pi$ \\
\hline Elkhart SMSA & 140,000 & $58 \mathrm{~km}$ & $S F$ \\
\hline Mishiwaka & 40,000 & $47 \mathrm{~km}$ & SE \\
\hline Michigan City & 37,000 & $40 \mathrm{~km}$ & SW \\
\hline
\end{tabular}

SITE-SPECIFIC DATA - AIRBORNE PATHWAYS

Average Annual State Production

Veg: $1.1 \mathrm{E} 8$ kilogram

of Crops and Animal Products

In 80-km Radius Circle

Milk: $2.3 \mathrm{E} 8$ liter

Meat: $1.9 \mathrm{E} 8$ kilogram

Regiona? Productivity Factor:

Animal Grazing Factor:

0.6

0.5

Meteorology Period of Record: I MAY 75 - 3.. APR 76 Recovery: 95\%

SITE-SPECIFIC DATA - WATERBORNE PATHWAYS $v " a$ LAKE MICHIGAN

Average Dilution Flow

from Plant: $3,300 \mathrm{ft}^{3} / \mathrm{s}$

Drinking Water:

Exposed Population: 250 , 900

Dilution Factor: 0.025

Fish:

Edible Harvest: $1.5 \mathrm{E} 6 \mathrm{~kg} / \mathrm{yr}$

Dilution Factor: 0.01

(a) Drinking water dilution factor estimated by averaging dilution factors derived from FES (1973) suitabiy weighted for population. 
POPULATION DOSE-COMMITMENT ESTIMATES AND

AVERAGE INDIVIDUAL DOSE-COMMITMENT HISTOGRAM FOR

COOK 1 AND 2

Dose Commitments (person-rem) from Waterborne Pathways

\begin{tabular}{|c|c|c|c|c|c|}
\hline & Total Body & GI-LLI & Thyroid & Bone & Liver \\
\hline $\begin{array}{l}\text { Infant } \\
\text { Child } \\
\text { Teen } \\
\text { Adult }\end{array}$ & $\begin{array}{l}2.3 \mathrm{E}-03 \\
3.3 \mathrm{E}-02 \\
2.4 \mathrm{E}-02 \\
2.4 \mathrm{E}-01\end{array}$ & $\begin{array}{l}2.3 \mathrm{E}-03 \\
2.8 \mathrm{E}-02 \\
1.3 \mathrm{E}-02 \\
1.2 \mathrm{E}-01\end{array}$ & $\begin{array}{l}3.1 \mathrm{E}-03 \\
3.1 \mathrm{E}-02 \\
1.2 \mathrm{E}-02 \\
9.5 \mathrm{E}-02\end{array}$ & $\begin{array}{l}4.9 \mathrm{E}-\mathrm{D} 4 \\
3.9 \mathrm{E}-02 \\
2.2 \mathrm{E}-02 \\
1.3 \mathrm{E}-02\end{array}$ & $\begin{array}{l}2.9 \mathrm{E}-03 \\
7.0 \mathrm{E}-02 \\
4.5 \mathrm{E}-02 \\
2.9 \mathrm{E}-01\end{array}$ \\
\hline OTAL & $2.9 \mathrm{E}-01$ & $1.6 \mathrm{E}-01$ & $1.4 \mathrm{E}-01$ & $1.9 E-01$ & 4. $1 \mathrm{E}-01$ \\
\hline
\end{tabular}

Dose Commitments (person-rem) from Airborne Pathways

Total Body GI-LLI Thyroid Bone Liver Lung

$\begin{array}{lllllll}\text { Infant } & 7.2 \mathrm{E}-04 & 6.9 \mathrm{E}-04 & 6.5 \mathrm{E}-03 & 7.9 \mathrm{E}-04 & 9.8 \mathrm{E}-04 & 7.7 \mathrm{E}-04 \\ \text { Child } & 8.8 \mathrm{E}-03 & 8.3 \mathrm{E}-03 & 4.7 \mathrm{E}-02 & 8.6 \mathrm{E}-03 & 1.1 \mathrm{E}-02 & 9.4 \mathrm{E}-03 \\ \text { Teen } & 6.3 \mathrm{E}-03 & 6.0 \mathrm{E}-03 & 2.1 \mathrm{E}-02 & 5.4 \mathrm{E}-03 & 6.9 \mathrm{E}-03 & 7.0 \mathrm{E}-03 \\ \text { Adult } & 3.7 \mathrm{E}-02 & 3.6 \mathrm{E}-02 & 8.5 \mathrm{E}-02 & 3.1 \mathrm{E}-02 & 3.8 \mathrm{E}-02 & 3.9 \mathrm{E}-02 \\ \text { TOTAL } & 5.3 \mathrm{E}-02 & 5.1 \mathrm{E}-02 & 1.6 \mathrm{E}-01 & 4.5 \mathrm{E}-02 & 5.7 \mathrm{E}-02 & 5.6 \mathrm{E}-02\end{array}$

Production/Consumption factors:

Produce: $<1$ Milk: $<1 \quad$ Meat: 1.2

Fraction of Population Receiving an Average Individual

Total-Budy Dose Commitment from Airborne Pathways

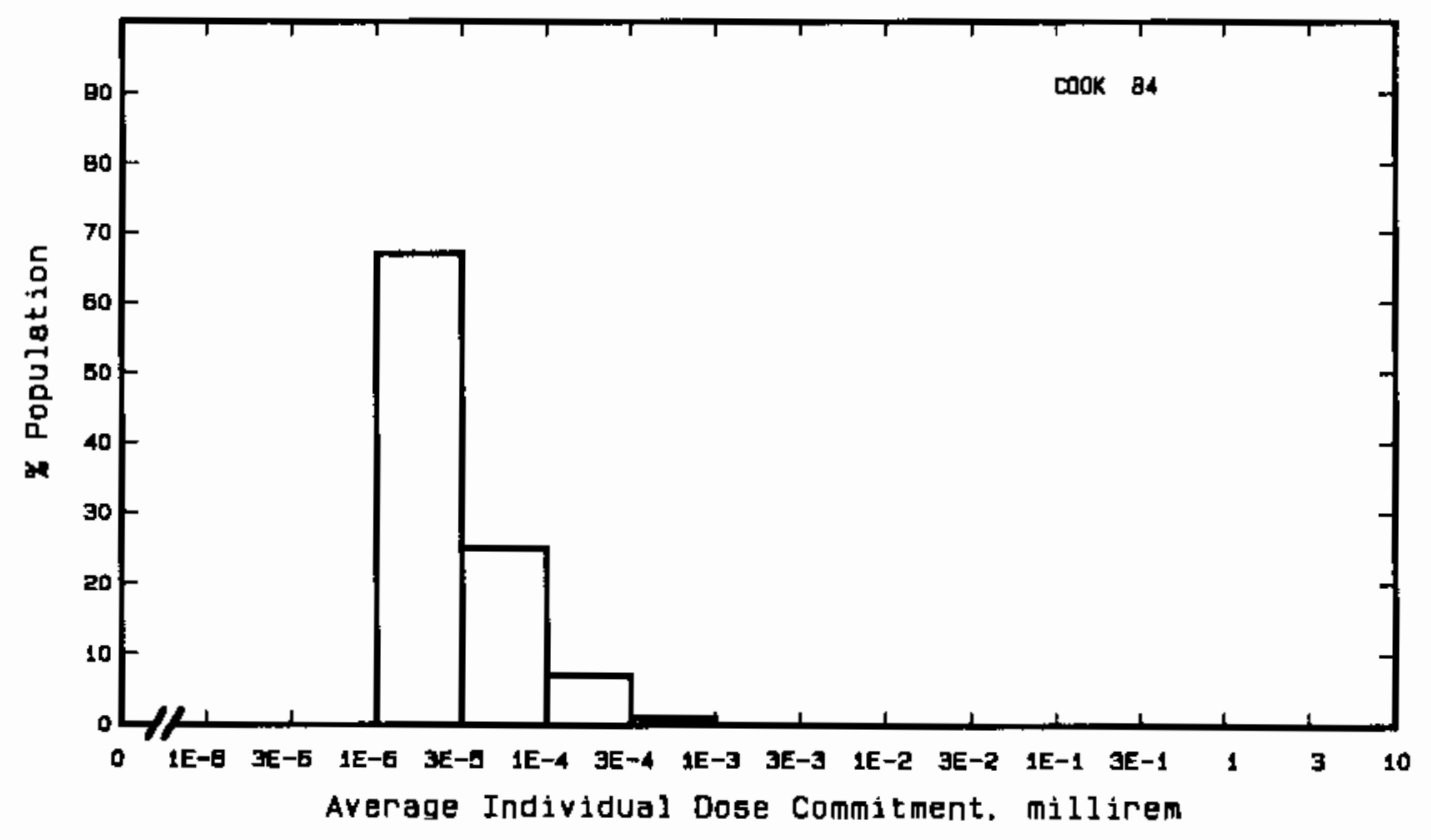


Site: COOPER

NEMAHA COUNTY, NEBRASKA

Location: $\quad$ N $40.3619^{\circ} \quad$ W $95.6411^{\circ}$

POPULATION DATA

Total Population Within 2-to-80-km Region: 1.7E5

Major Metropolitan Centers Within Region:

Center
Nebraska City
Red Oak
Plattsmouth
Shenandoah
Falls City

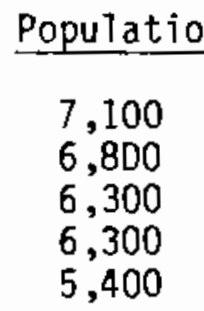

Location

$40 \mathrm{~km} \quad \mathrm{NNW}$

$80 \mathrm{~km} \quad \mathrm{NNE}$

$76 \mathrm{~km} \quad \mathrm{NNW}$

$51 \mathrm{~km} \quad$ NNE

Falls City

5,400

$33 \mathrm{~km} \quad \mathrm{~s}$

\section{SITE-SPECIFIC DATA - AIRBORNE PATHWAYS}

Average Annual State Production

of Crops and Animal Products

In 80-km Radius Circle

Regional Productivity Factor:

Animal Grazing Factor:

Meteorology Period of Record: I MAR 70 - 31 DEC 75 Recovery: 89\%
Veg: $9.7 E 7$ kilogram

MiTk: $7.2 \mathrm{E} 7$ liter

Meat: 2.0E8 kilogram

1

0.6

SITE-SPECIFIC DATA - WATERBORNE PATHWAYS via MISSOURI RIVER

Average River Flow at Site: $31,000 \mathrm{ft}^{3} / \mathrm{s}$

Drinking water:

Exposed Population: None

Fish:

Edible Harvest: $5.0 \mathrm{E} 3^{(\mathrm{a})} \mathrm{kg} / \mathrm{yr}$
Dilution Factor: $0.5^{(\mathrm{a})}$

(a) Assumes $1 / 2$ fish harvest caught below plant. 


\section{POPULATION DOSE-COMMITMENT ESTIMATES AND \\ AVERAGE INDIVIDUAL DOSE-COMMITMENT HISTOGRAM FOR \\ COOPER}

Dose Commitments (person-rem) from Waterborne Pathways

\begin{tabular}{|c|c|c|c|c|c|}
\hline & Total Body & GI-LLI & Thyroid & Bone & Liver \\
\hline $\begin{array}{l}\text { Infant } \\
\text { Child } \\
\text { Teen } \\
\text { Adult }\end{array}$ & $\begin{array}{l}0.0 \mathrm{E}+00 \\
2.3 \mathrm{E}-03 \\
4.5 \mathrm{E}-03 \\
4.9 \mathrm{E}-02\end{array}$ & $\begin{array}{l}0.0 \mathrm{E}+00 \\
1.3 \mathrm{E}-04 \\
2.6 \mathrm{E}-04 \\
2.2 \mathrm{E}-03\end{array}$ & $\begin{array}{l}0.0 \mathrm{E}+00 \\
6.0 \mathrm{E}-06 \\
4.3 \mathrm{E}-06 \\
2.8 \mathrm{E}-05\end{array}$ & $\begin{array}{l}0 . \mathrm{DE}+00 \\
1.1 \mathrm{E}-02 \\
6.6 \mathrm{E}-03 \\
3.8 \mathrm{E}-02\end{array}$ & $\begin{array}{l}0.0 \mathrm{E}+00 \\
1.3 \mathrm{E}-02 \\
1.1 \mathrm{E}-02 \\
6.7 \mathrm{E}-02\end{array}$ \\
\hline TOTAL & $5.6 \mathrm{E}-02$ & $2.6 E-03$ & $3.9 \mathrm{E}-05$ & $5.6 E-02$ & 9. $1 \mathrm{E}-02$ \\
\hline
\end{tabular}

Dose Commitments (person-rem) from Airborne Pathways

Total Body GI-LLI Thyroid Bone Liver Lung

$\begin{array}{lllllll}\text { Infant } & 1.9 \mathrm{E}-04 & 1.9 \mathrm{E}-04 & 1.2 \mathrm{E}-03 & 2.0 \mathrm{E}-04 & 2.1 \mathrm{E}-04 & 1.9 \mathrm{E}-04 \\ \text { Child } & 2.2 \mathrm{E}-03 & 2 . \mathrm{IE}-03 & 1.2 \mathrm{E}-02 & 2.7 \mathrm{E}-03 & 2.4 \mathrm{E}-03 & 2.2 \mathrm{E}-03 \\ \text { Teen } & 1.6 \mathrm{E}-03 & 1.5 \mathrm{E}-03 & 5.2 \mathrm{E}-03 & 1.7 \mathrm{E}-03 & 1.7 \mathrm{E}-03 & 1.7 \mathrm{E}-03 \\ \text { Adult } & 9.6 \mathrm{E}-03 & 9.3 \mathrm{E}-03 & 2.2 \mathrm{E}-02 & 9.8 \mathrm{E}-03 & 9.6 \mathrm{E}-03 & 9.6 \mathrm{E}-03 \\ \text { TOTAL } & 1.4 \mathrm{E}-02 & 1.3 \mathrm{E}-02 & 4.0 \mathrm{E}-02 & 1.4 \mathrm{E}-02 & 1.4 \mathrm{E}-02 & 1.4 \mathrm{E}-02\end{array}$

Production/Consumption factors:

Produce: $2.9 \quad$ Milk: 3.2 Meat: 14

Fraction of Population Receiving an Average Individual

Tota1-Body Dose Commitment from Airborne Pathways

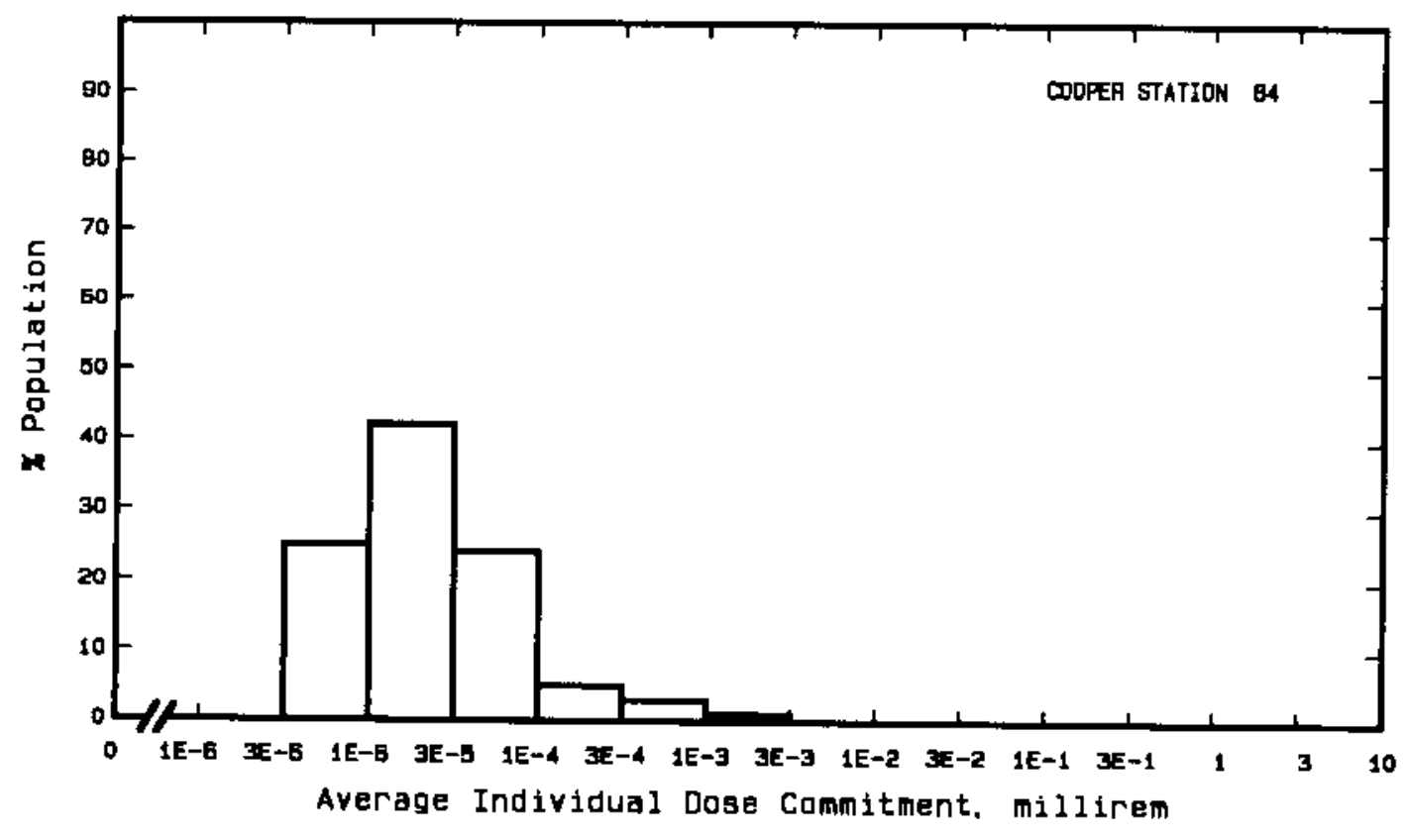


Site: CRYSTAL RIVER

CRYSTAL RIVER, FLORIDA

Location: $\quad$ N $28.9572^{\circ} \quad$ W $82.6989^{\circ}$

POPULATION DATA

Total Population Within 2-to-80-km Region: $4.0 \mathrm{E} 5$

Major Metropolitan Centers Within Region:

\begin{tabular}{lccl} 
Center & Population & \multicolumn{2}{c}{ Location } \\
\cline { 2 - 3 } & 41,000 & $60 \mathrm{~km}$ & ENE \\
Ocala & 14,000 & $80 \mathrm{~km}$ & $\mathrm{E}$ \\
Leesburg & 12,000 & $79 \mathrm{~km}$ & $\mathrm{~S}$
\end{tabular}

SITE-SPECIFIC DATA - AIRBORNE PATHWAYS

Average Annual State Production

of Crops and Animal Products

In 80-km Radius Circle

Veg: $2.8 \mathrm{E} 7 \mathrm{kilogram}$

Milk: 1.1 E8 liter

Meat: $7.2 \mathrm{E} 7$ kilogram

Regiona? Productivity Factor:

Animal Grazing Factor:

0.5

1

Meteorology Period of Record: 1 JAN 75 - 31 DEC 75 Recovery: 93\%

SITE-SPECIFIC DATA - WATERBORNE PATHWAYS via GULF Of MEXICO

Average Dilution Flow

from Plant: $2.2 \mathrm{ft}^{3} / \mathrm{s}$

Fish:

Edible Harvest: $3.2 \mathrm{E} 5 \mathrm{~kg} / \mathrm{yr}$

Dilution Factor: 0.1

Invertebrates:

Edible Harvest: $1.8 \mathrm{E} 5 \mathrm{~kg} / \mathrm{yr}$

Dilution Factor: 0.1 


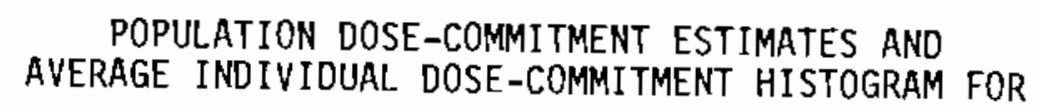

CRYSTAL RIVER

Dose Commitments (person-ren) from Waterborne Pathways

\begin{tabular}{|c|c|c|c|c|c|}
\hline & Total Body & GI-LLI & Thyroid & Bone & Liver \\
\hline $\begin{array}{l}\text { Infant } \\
\text { Child } \\
\text { Teen } \\
\text { Adult }\end{array}$ & $\begin{array}{l}0.0 \mathrm{E}+00 \\
9.0 \mathrm{E}-01 \\
7.1 \mathrm{E}-01 \\
5.2 \mathrm{E}+00\end{array}$ & $\begin{array}{l}0.0 \mathrm{E}+00 \\
8.5 \mathrm{E}+00 \\
1.8 \mathrm{E}+01 \\
1.6 \mathrm{E}+02\end{array}$ & $\begin{array}{l}0.0 \mathrm{E}+00 \\
3.8 \mathrm{E}-01 \\
2.9 \mathrm{E}-01 \\
2.0 \mathrm{E}+00\end{array}$ & $\begin{array}{l}0.0 \mathrm{E}+00 \\
2.2 \mathrm{E}+00 \\
1.3 \mathrm{E}+00 \\
7.5 \mathrm{E}+00\end{array}$ & $\begin{array}{l}0.0 E+00 \\
1.7 E+00 \\
1.4 E+00 \\
8.3 E+00\end{array}$ \\
\hline TOTAL & $6.8 \mathrm{E}+00$ & $1.9 E+02$ & $2.7 E+00$ & $1.1 E+01$ & $1.1 E+01$ \\
\hline
\end{tabular}

Dose Commitments (person-rem) from Airborne Pathways Total Body GI-LLI Thyroid Bone Liver Lung

$\begin{array}{lllllll}\text { Infant } & 1.3 \mathrm{E}-04 & 1.3 \mathrm{E}-04 & 1 . \mathrm{BE}-04 & 1.1 \mathrm{E}-04 & 1.3 \mathrm{E}-04 & 1.4 \mathrm{E}-04 \\ \text { ChiTd } & 1.5 \mathrm{E}-03 & 1.5 \mathrm{E}-03 & 1.8 \mathrm{E}-03 & 1.3 \mathrm{E}-03 & 1.5 \mathrm{E}-03 & 1.6 \mathrm{E}-03 \\ \text { Teen } & 1.1 \mathrm{E}-03 & 1.1 \mathrm{E}-03 & 1.2 \mathrm{E}-03 & 9.3 \mathrm{E}-04 & 1.1 \mathrm{E}-03 & 1.2 \mathrm{E}-03 \\ \text { AduTt } & 6.3 \mathrm{E}-03 & 6.3 \mathrm{E}-03 & 6.7 \mathrm{E}-03 & 5.6 \mathrm{E}-03 & 6.3 \mathrm{E}-03 & 6.8 \mathrm{E}-03 \\ \text { TOTAL } & 9.0 \mathrm{E}-03 & 9.0 \mathrm{E}-03 & 9.8 \mathrm{E}-03 & 7.9 \mathrm{E}-03 & 9.1 \mathrm{E}-03 & 9.7 \mathrm{E}-03\end{array}$

Production/Consumption factors:

Produce: <1 Milk: 1.0 Meat: 1.1

Fraction of Population Receiving an Average Individual Total-Body dose Commitment from Airborne Pathways

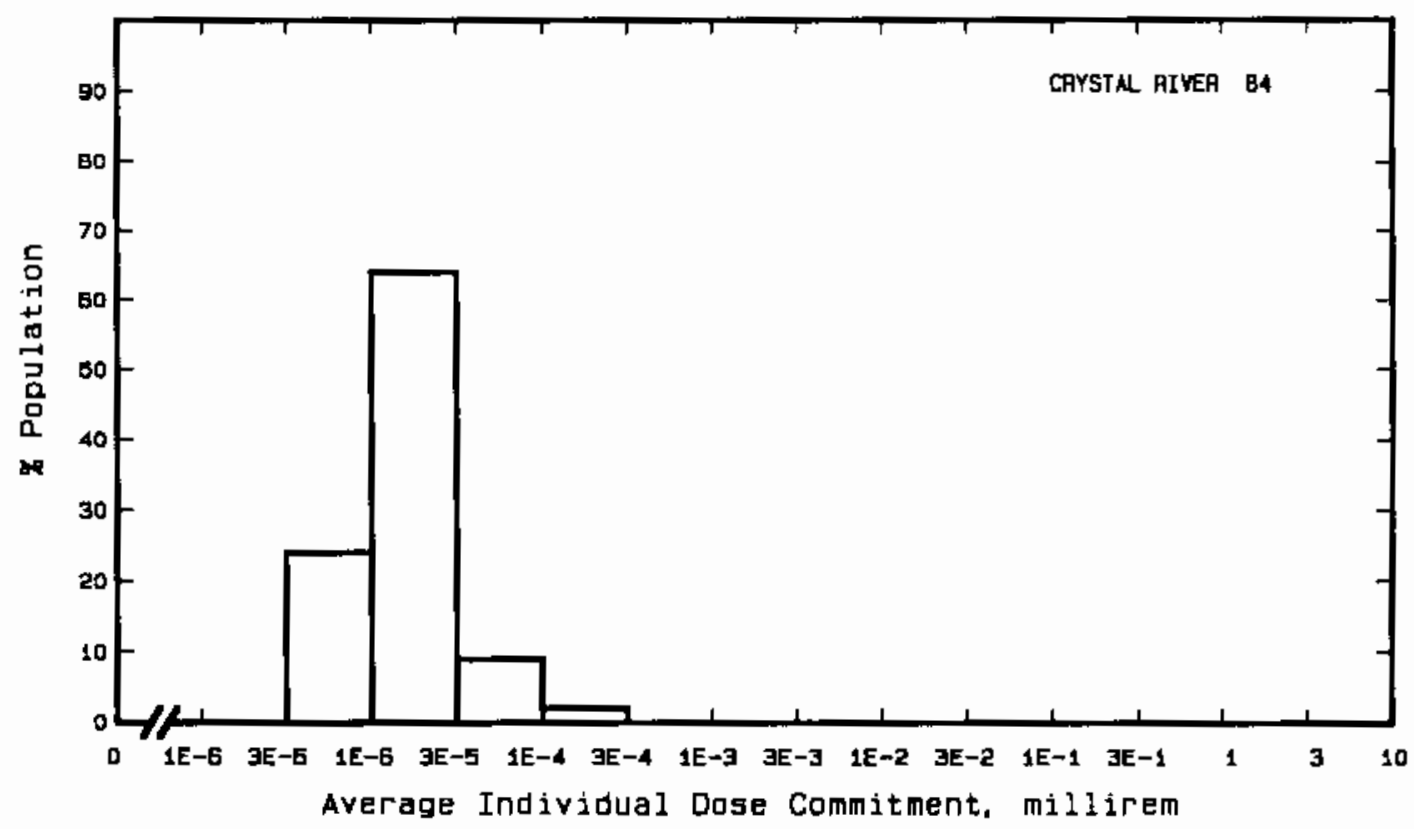


Site: DAVIS-BESSE

PORT CLINTON, OHIO

Location: $\quad \mathrm{N} 41.5972^{\circ} \quad$ W $83.08154^{\circ}$

POPULATION DATA

Total Population Within 2-to-80-km Region: $1.8 \mathrm{E} 6$

Major Metropolitan Centers Within Region:

Center
Toledo SMSA
Dearborn
Taylor
Lorain
Lincoln Park
Findlay
Sandusky

Population

Location

790,000

91,000

77,000

75,000

45,000

36,000

33,000

$38 \mathrm{~km} \quad W N W$

$80 \mathrm{~km} \quad \mathrm{~N}$

$71 \mathrm{~km} \quad \mathrm{~N}$

$77 \mathrm{~km} \quad \mathrm{ESE}$

$73 \mathrm{~km} \mathrm{~N}$

$77 \mathrm{~km} \quad \mathrm{SW}$

$35 \mathrm{~km} \quad \mathrm{ESE}$

\section{SITE-SPECIFIC DATA - AIRBORNE PATHWAYS}

Average Annual State Production

of Crops and Animal Products

In 80-km Radius Circle

Regional Productivity Factor:

Animal Grazing Factor:

Meteorology Period of Record: 4 AUG 74 - 3 AUG 76 Recovery: 99\%

Veg: $6.9 \mathrm{E} 7 \mathrm{ki}$ Togram

Milk: $3.7 \mathrm{~EB}$ liter

Meat: $1.2 \mathrm{~EB}$ kilogram

0.5

0.5

SITE-SPECIFIC DATA - WATERBORNE PATHWAYS via LAKE ERIE

Average Dilution Flow

from Plant: $30 \mathrm{ft}^{3} / \mathrm{s}$

Orinking Water:

Exposed Population: $450 \mathrm{p} 090$
Dilution Factor: $2.0 \mathrm{E}-4 \mathrm{P}$

Fish:

Edible Harvest: $5.7 \mathrm{E}^{(\mathrm{b})} \mathrm{kg} / \mathrm{yr}$

Dilution Factor: $1.8 \mathrm{E}-4{ }^{(b)}$

(a) Drinking water dilution factor estimated by averaging dilution factor derived from FES (1973), suitably weighted for population.

(b) Letter from Terry D. Murray, Toledo Edison Company to Charles A. Willis, NRC, July 20, 1984. 
POPULATION DOSE-COMMITMENT ESTIMATES AND

AVERAGE INDIVIOUAL DOSE-COMMITMENT HISTOGRAM FOR

DAVIS-BESSE

Dose Commitments (person-rem) from Waterborne Pathways

Total Body GI-LLI Thyroid Bone Liver

$\begin{array}{llllll}\text { Infant } & 2.7 \mathrm{E}-04 & 2.6 \mathrm{E}-04 & 3.2 \mathrm{E}-04 & 1.9 \mathrm{E}-04 & 4.9 \mathrm{E}-04 \\ \text { Child } & 1.8 \mathrm{E}-02 & 4.0 \mathrm{E}-03 & 3.4 \mathrm{E}-03 & 8.4 \mathrm{E}-02 & 9.3 \mathrm{E}-02 \\ \text { Teen } & 3.0 \mathrm{E}-02 & 3.1 \mathrm{E}-03 & 1.3 \mathrm{E}-03 & 4.9 \mathrm{E}-02 & 7.6 \mathrm{E}-02 \\ \text { Adult } & 3.2 \mathrm{E}-01 & 2.6 \mathrm{E}-02 & 1.1 \mathrm{E}-02 & 2.8 \mathrm{E}-01 & 4.5 \mathrm{E}-01 \\ \text { TOTAL } & 3.7 \mathrm{E}-01 & 3.4 \mathrm{E}-02 & 1.6 \mathrm{E}-02 & 4.2 \mathrm{E}-01 & 6.2 \mathrm{E}-01\end{array}$

Dose Commitments (person-rem) from Airborne Pathways

Total Body GI-LLI Thyroid Bone Liver Lung

$\begin{array}{lllllll}\text { Infant } & 1.5 \mathrm{E}-04 & 1.5 \mathrm{E}-04 & 1.7 \mathrm{E}-03 & 1.0 \mathrm{E}-04 & 1.6 \mathrm{E}-04 & 1.6 \mathrm{E}-04 \\ \text { ChiTd } & 1.8 \mathrm{E}-03 & 1 . \mathrm{BE}-03 & 1.0 \mathrm{E}-02 & 1.1 \mathrm{E}-03 & 1.8 \mathrm{E}-03 & 1.9 \mathrm{E}-03 \\ \text { Teen } & 1.2 \mathrm{E}-03 & 1.2 \mathrm{E}-03 & 4.4 \mathrm{E}-03 & 8.2 \mathrm{E}-04 & 1.2 \mathrm{E}-03 & 1.4 \mathrm{E}-03 \\ \text { Adult } & 7.0 \mathrm{E}-03 & 7.0 \mathrm{E}-03 & 1.7 \mathrm{E}-02 & 5.0 \mathrm{E}-03 & 7.0 \mathrm{E}-03 & 7.6 \mathrm{E}-03 \\ \text { TOTAL } & 1.0 \mathrm{E}-02 & 1.0 \mathrm{E}-02 & 3.3 \mathrm{E}-02 & 7.0 \mathrm{E}-03 & 1.0 \mathrm{E}-02 & 1.1 \mathrm{E}-02\end{array}$

Production/Consumption factors:

Produce: <1 Milk: <1 Meat: <1

Fraction of Population Receiving an Average Individual

Total-Eody Dose Commitment from Airborne Pathways

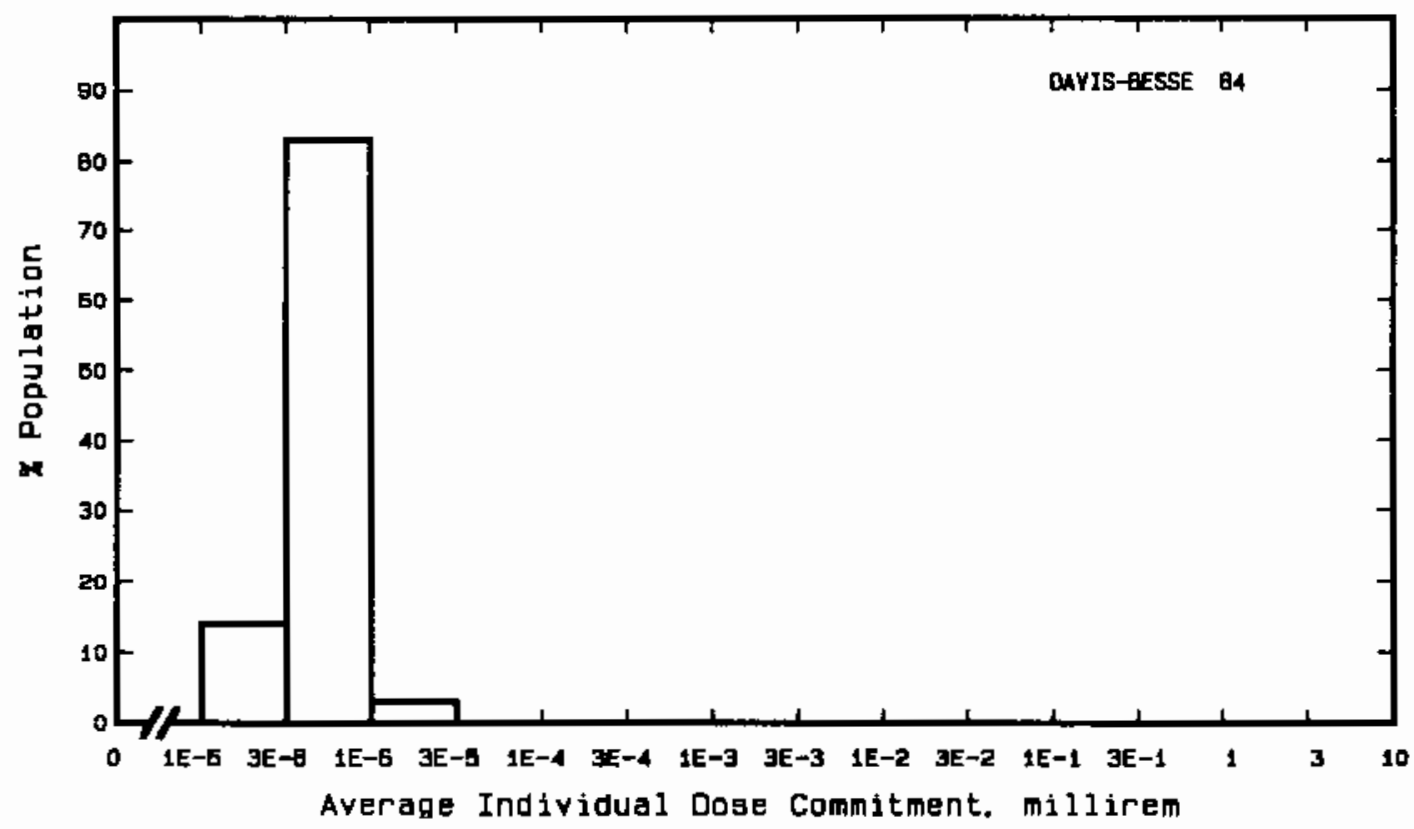


Site: DIABLO CANYON

Location: N $35.2111^{\circ}$
AVILA BEACH, CALIFORNIA

W $120.8522^{\circ}$

POPULATION DATA

Total PopuTation Within 2-to-80-km Region: $2.8 \mathrm{E5}$

Major Metropolitan Centers Within Region:

\begin{tabular}{lcccc} 
Center & Population & \multicolumn{3}{c}{ Location } \\
\cline { 2 - 3 } & & & & \\
San Luis 0bispo & 37,000 & $34 \mathrm{~km}$ & ENE \\
Atascudero & 13,000 & $74 \mathrm{~km}$ & SNE \\
Lompoc & 25,000 & $18 \mathrm{~km}$ & N
\end{tabular}

SITE-SPECIFIC DATA - AIRBORNE PATHWAYS

Average Annual State Production

of Crops and Animat Products

In $80-\mathrm{km}$ Radius Circle

Regional Productivity Factor:

Anima1 Grazing Factor:

Meteorology Period of Record: 1 JAN 84 - 31 DEC 84 Recovery: 98\%
Veg: $4.7 E 7$ kilogram

Milk: 2.3E8 liter

Meat: $5.0 E 7$ kilogram

0.5

1

SITE-SPECIFIC DATA - WATER8ORNE PATHWAYS via Pacific Ocean

Average Dilution Flow

from Plant: $220 \mathrm{ft}^{3} / \mathrm{s}$

Fish:

Edible Harvest: $2.0 \mathrm{E} 6 \mathrm{~kg} / \mathrm{yr}$

Dilution Factor: 0.001

Invertebrates:

Edible Harvest: None 
POPULATION DOSE-COMMITMENT ESTIMATES AND

AVERAGE INDIVIDUAL DOSE-COMMITMENT HISTOGRAM FOR

DIABLO CANYON

Dose Commitments (person-rem) from Waterborne Pathways

\begin{tabular}{|c|c|c|c|c|c|}
\hline & Total Body & GI -LLI & Thyroid & Bone & Liver \\
\hline $\begin{array}{l}\text { Infant } \\
\text { Child } \\
\text { Teen } \\
\text { Adult }\end{array}$ & $\begin{array}{l}0.0 \mathrm{E}+00 \\
2.7 \mathrm{E}-06 \\
1.9 \mathrm{E}-06 \\
1.2 \mathrm{E}-05\end{array}$ & $\begin{array}{l}0.0 \mathrm{E}+00 \\
5.5 \mathrm{E}-06 \\
1.2 \mathrm{E}-05 \\
1.0 \mathrm{E}-04\end{array}$ & $\begin{array}{l}0.0 \mathrm{E}+00 \\
5.9 \mathrm{E}-07 \\
4.4 \mathrm{E}-07 \\
3.0 \mathrm{E}-06\end{array}$ & $\begin{array}{l}0.0 \mathrm{E}+00 \\
9.0 \mathrm{E}-07 \\
5.6 \mathrm{E}-07 \\
3.4 \mathrm{E}-06\end{array}$ & $\begin{array}{l}0.0 \mathrm{E}+00 \\
2.9 \mathrm{E}-06 \\
2.7 \mathrm{E}-06 \\
1.6 \mathrm{E}-05\end{array}$ \\
\hline TOTAL & $1.6 \mathrm{E}-05$ & $1.2 E-04$ & $4.0 \mathrm{E}-06$ & $4.8 \mathrm{E}-06$ & $2.2 E-05$ \\
\hline
\end{tabular}

Dose Commitments (person-rem) from Airborne Pathways

Total Body GI-LLI Thyroid Bone Liver Lung

$\begin{array}{lllllll}\text { Infant } & 1.4 \mathrm{E}-07 & 7.4 \mathrm{E}-08 & 6.8 \mathrm{E}-08 & 5.2 \mathrm{E}-07 & 6.8 \mathrm{E}-08 & 1.3 \mathrm{E}-07 \\ \text { Child } & 5.8 \mathrm{E}-06 & 1.3 \mathrm{E}-06 & 9.1 \mathrm{E}-07 & 2.5 \mathrm{E}-05 & 9.1 \mathrm{E}-07 & 1.8 \mathrm{E}-06 \\ \text { Teen } & 2.8 \mathrm{E}-06 & 9.5 \mathrm{E}-07 & 6.3 \mathrm{E}-07 & 1.2 \mathrm{E}-05 & 6.3 \mathrm{E}-07 & 1.3 \mathrm{E}-06 \\ \text { Adult } & 1.3 \mathrm{E}-05 & 5.2 \mathrm{E}-06 & 3.7 \mathrm{E}-06 & 5.7 \mathrm{E}-05 & 3.7 \mathrm{E}-06 & 6.2 \mathrm{E}-06 \\ \text { TOTAL } & 2.2 \mathrm{E}-05 & 7.5 \mathrm{E}-06 & 5.3 \mathrm{E}-06 & 9.5 \mathrm{E}-05 & 5.3 \mathrm{E}-06 & 9.4 \mathrm{E}-06\end{array}$

Production/Consumption factors:

Produce: <1 Milk: 3.2 Meat: I.I

Fraction of Population Receiving an Average Individual

Total-Body Dose Commitment from Airborne Pathways

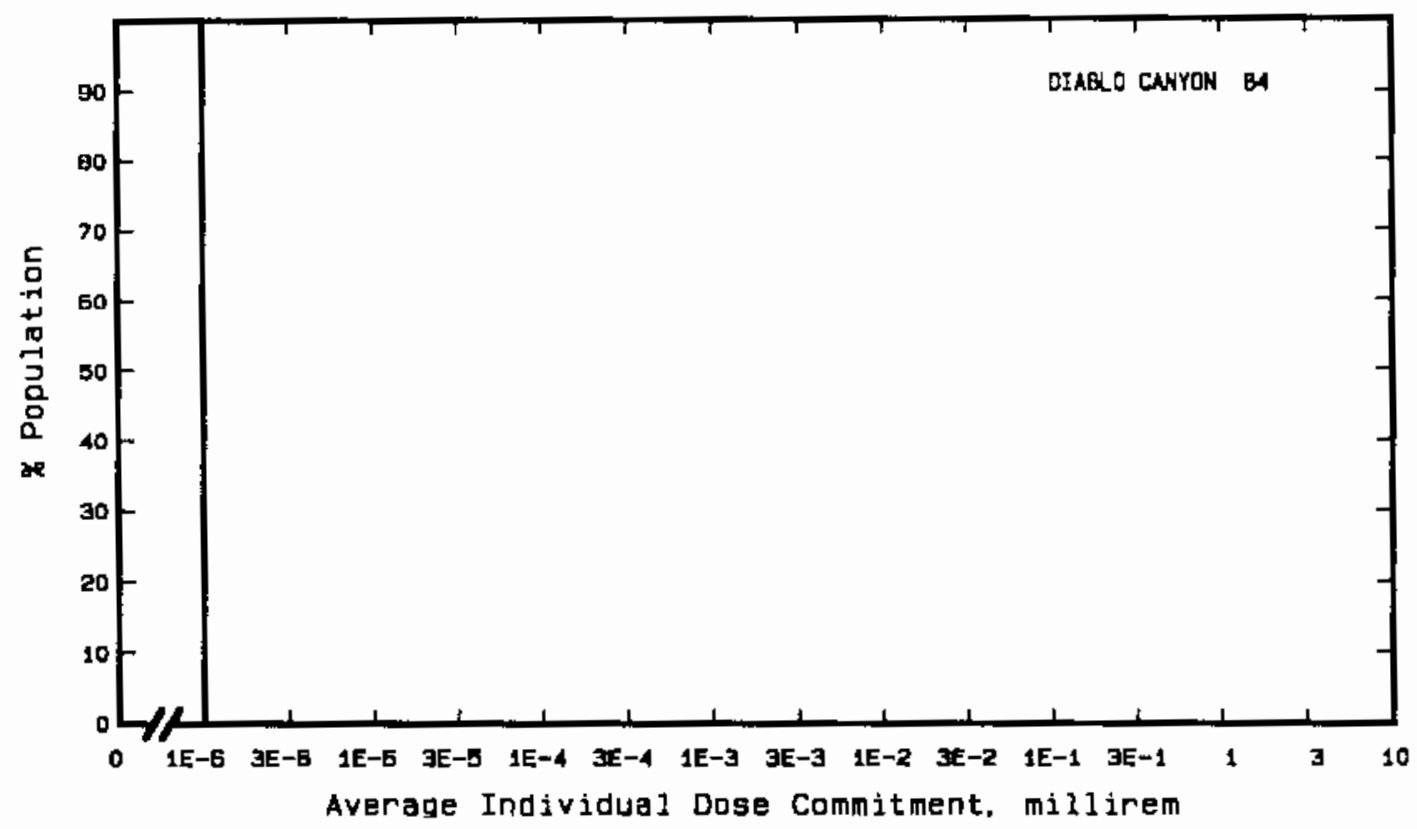


Site: DRESDEN

GRUNDY COUNTY, ILLINOIS

Location: $\quad$ N $41.3897^{\circ} \quad$ W $88.2711^{\circ}$

POPULATION DATA

Total Population Within 2-to-80-km Region: 6.4E6

Major Metropolitan Centers Within Region:

\begin{tabular}{lcccc}
\multicolumn{1}{c}{ Center } & \multicolumn{2}{c}{ Population } & \multicolumn{2}{c}{ Location } \\
\cline { 2 - 6 } Chicago SMSA & $7,100,000(\mathrm{a})$ & & $75 \mathrm{~km}$ & $\mathrm{NE}$ \\
Gary-Hamanond-E.Chicago-SMSA & $640,000(\mathrm{a})$ & & $80 \mathrm{~km}$ & $\mathrm{ENE}$ \\
Kankakee SMSA & 100,000 & $45 \mathrm{~km}$ & SE \\
Aurora & 81,000 & $41 \mathrm{~km}$ & $\mathrm{~N}$ \\
Joliet & 78,000 & $22 \mathrm{~km}$ & $\mathrm{NE}$ \\
Elgin & 56,000 & $74 \mathrm{~km}$ & $\mathrm{~N}$
\end{tabular}

SITE-SPECIFIC DATA - AIRBORNE PATHWAYS

Average Annual State Production

of Crops and Animal Products

In $80-\mathrm{km}$ Radius Circle

Regional Productivity Factor:

Animal Grazing Factor:
Veg: $1.1 \mathrm{E} 8 \mathrm{kilogram}$

MiTk: 1.8E8 liter

Meat: $1.9 E 8$ kilogram

1

0.5

Meteorology Period of Record: I JAN $74-31$ JAN 75 Recovery: 77\%

SITE-SPECIFIC DATA - WATERBORNE PATHWAYS via ILLINOIS RIVER

Average River Flow

at Site: $12,000 \mathrm{ft}^{3} / \mathrm{s}$

Drinking Water:

Exposed Poputation: (b)

Fish:

Edible Harvest: (b) $\mathrm{kg} / \mathrm{yr}$

(a) Population of total SMSA given; population of SMSA fraction within $80 \mathrm{~km}$ of site would be somewhat smatler.

(b) River water used for sewage disposal for Chicago, so population doses from Tiquid pathways assumed to be near zero. 


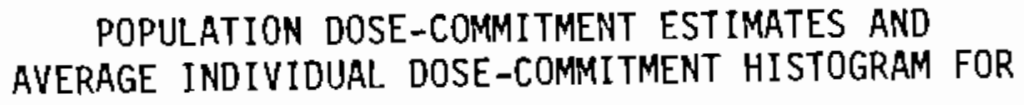

DRESDEN 1,2 AND 3

Dose Commitments (person-rem) from Waterborne Pathways

Total Body GI-LLI Thyroid Bone Liver

Infant

Child

Teen

Adult

(Little or No Waterborne Pathway Doses)

TOTAL

Production/Consumption factors:

Produce: $<1$ Milk: <1 Meat: <1

Fraction of Population Receiving an Average Individual

Total-Body Dose Commitment from Airborne Pathways

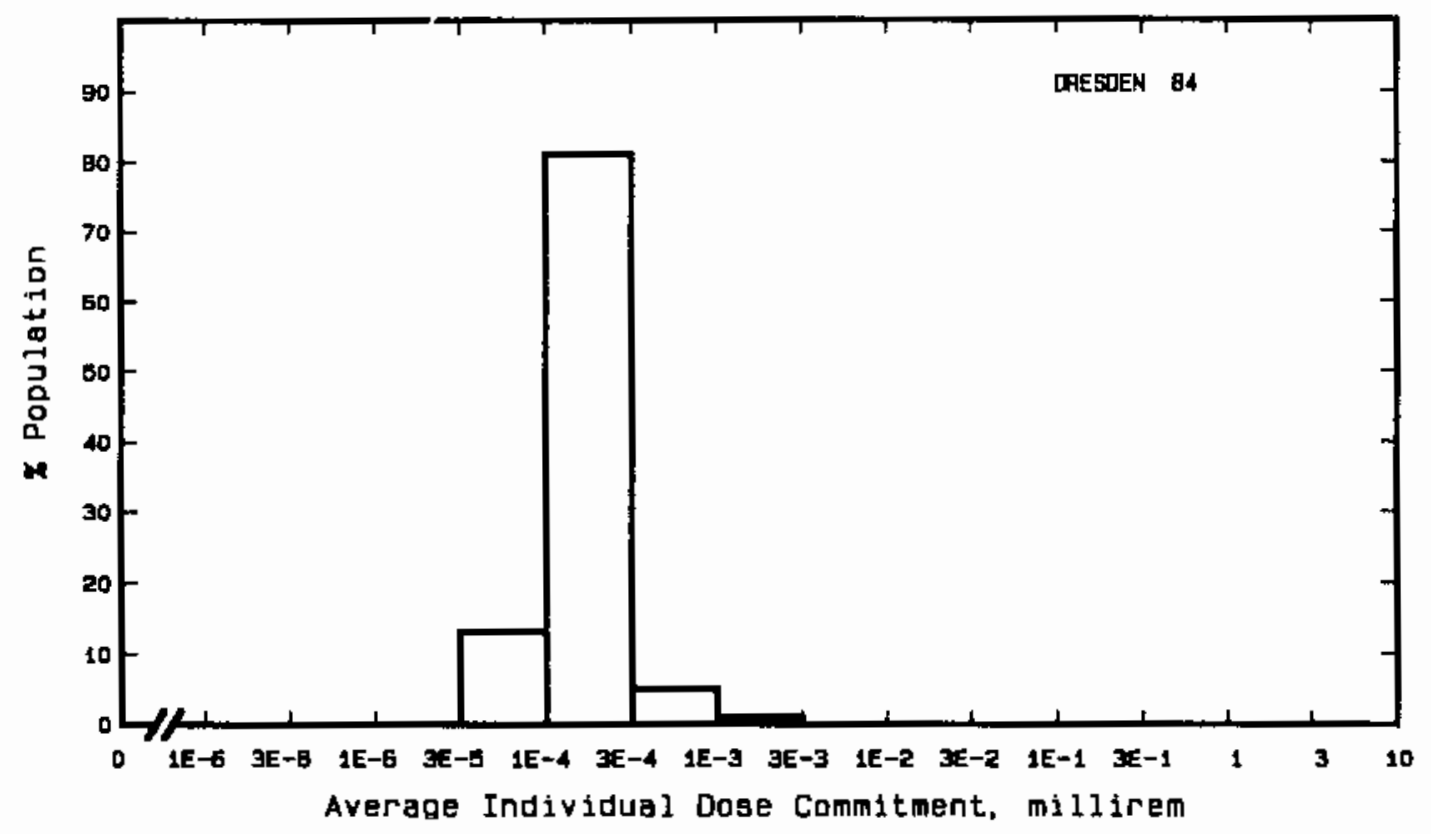


Site: DUANE ARNOLD

CEDAR RAPIDS, IOWA

Location: $\quad \mathrm{N} 42.1006^{\circ} \quad$ W $91.7772^{\circ}$

POPULATION DATA

TotaT Population Within 2-to-80-km Region: 6.0E5

Major Metropolitan Centers Within Region:

\begin{tabular}{lrrrr}
\multicolumn{2}{c}{ Center } & Population & \multicolumn{2}{c}{ Location } \\
\cline { 2 - 5 } Cedar Rapids SMSA & 170,000 & $17 \mathrm{~km}$ & SE \\
Waterloo-Cedar Falls SMSA & 140,000 & $64 \mathrm{~km}$ & NW \\
Iowa City SMSA & 82,000 & $52 \mathrm{~km}$ & SSE \\
Marion & 19,000 & $16 \mathrm{~km}$ & ESE
\end{tabular}

SITE-SPECIFIC DATA - AIRBORNE PATHWAYS

Average Annual State Production

Veg: $9.8 \mathrm{E} 7 \mathrm{ki}$ logram

of Crops and Animal Products

In 80-km Radius Circle

Milk: 2.6E8 liter

Meat: $4.2 E 8$ kilogram

Regional Productivity Factor:

AnimaT Grazing Factor:

1

0.5

Meteorology Period of Record: 1 FEB 71 - 31 DEC 75 Recovery: 57\%

SITE-SPECIFIC DATA - WATERBORNE PATHWAYS via CEDAR RIVER

Drinking Water:

Average River Flow

at Site: $3,100 \mathrm{ft}^{3} / \mathrm{s}$

Exposed Population: $170,000^{(a)}$

DiTution Factor: 1

Fish:

Edible Harvest: (b)

Dilution Factor: 1

(a) All persons in the Cedar Rapids SMSA assuned to drink river water.

(b) No fish catch data given in FES (1973); thus population was assumed to eat fish at generic consumption rates (Table A.1). 
POPULATION DOSE-COMMITMENT ESTIMATES AND

AVERAGE INDIVIDUAL DOSE-COMMITMENT HISTOGRAM FOR

DUANE ARNOLD

Dose Commitments (person-rem) from Waterborne Pathways

Total Body GI-LLI Thyroid Bone Liver

$\begin{array}{llllll}\text { Infant } & 7.1 \mathrm{E}-1 \mathrm{I} & 7.0 \mathrm{E}-11 & 6.5 \mathrm{E}-11 & 1.2 \mathrm{E}-11 & 6.7 \mathrm{E}-11 \\ \text { Child } & 9.0 \mathrm{E}-10 & 1.0 \mathrm{E}-09 & 7.4 \mathrm{E}-10 & 2.4 \mathrm{E}-10 & 7.9 \mathrm{E}-10 \\ \text { Teen } & 3.8 \mathrm{E}-10 & 7.2 \mathrm{E}-10 & 2.9 \mathrm{E}-10 & 1.1 \mathrm{E}-10 & 3.3 \mathrm{E}-10 \\ \text { Adult } & 3.0 \mathrm{E}-10 & 6.3 \mathrm{E}-09 & 2.5 \mathrm{E}-09 & 7.1 \mathrm{E}-10 & 2.7 \mathrm{E}-09 \\ \text { T0TAL } & 4.4 \mathrm{E}-09 & 8.1 \mathrm{E}-09 & 3.6 \mathrm{E}-09 & 1.1 \mathrm{E}-09 & 3.9 \mathrm{E}-09\end{array}$

Dose Commitments (person-rem) from Airborne Pathways

\begin{tabular}{|c|c|c|c|c|c|c|}
\hline & Tota 1 Body & GI-LLI & Thyroid & Bone & Liver & Lung \\
\hline $\begin{array}{l}\text { Infant } \\
\text { Chitd } \\
\text { Teen } \\
\text { Adult }\end{array}$ & $\begin{array}{l}4.6 \mathrm{E}-04 \\
5.3 \mathrm{E}-03 \\
3.8 \mathrm{E}-03 \\
2.3 \mathrm{E}-02\end{array}$ & $\begin{array}{l}4.6 \mathrm{E}-04 \\
5.4 \mathrm{E}-03 \\
3.9 \mathrm{E}-03 \\
2.4 \mathrm{E}-02\end{array}$ & $\begin{array}{l}1.1 \mathrm{E}-03 \\
1.2 \mathrm{E}-02 \\
6.4 \mathrm{E}-03 \\
3.2 \mathrm{E}-02\end{array}$ & $\begin{array}{l}4.4 \mathrm{E}-04 \\
5.1 \mathrm{E}-03 \\
3.6 \mathrm{E}-03 \\
2.2 \mathrm{E}-02\end{array}$ & $\begin{array}{l}4.6 \mathrm{E}-04 \\
5.3 \mathrm{E}-03 \\
3.8 \mathrm{E}-03 \\
2.3 \mathrm{E}-02\end{array}$ & $\begin{array}{l}4.8 \mathrm{E}-04 \\
5.7 \mathrm{E}-03 \\
4.2 \mathrm{E}-03 \\
2.5 \mathrm{E}-02\end{array}$ \\
\hline TAL & $3.2 \mathrm{E}-02$ & $3.3 E-02$ & $5.2 \mathrm{E}-02$ & $3.1 E-02$ & $3.2 E-02$ & $3.5 E-02$ \\
\hline
\end{tabular}

Production/Consumption factors:

Produce: 1 Milk: $3.3 \quad$ Meat: 8.7

Fraction of Population Recejving an Average Individual

Total-Bady Dose Commitment from Airborne Pathways

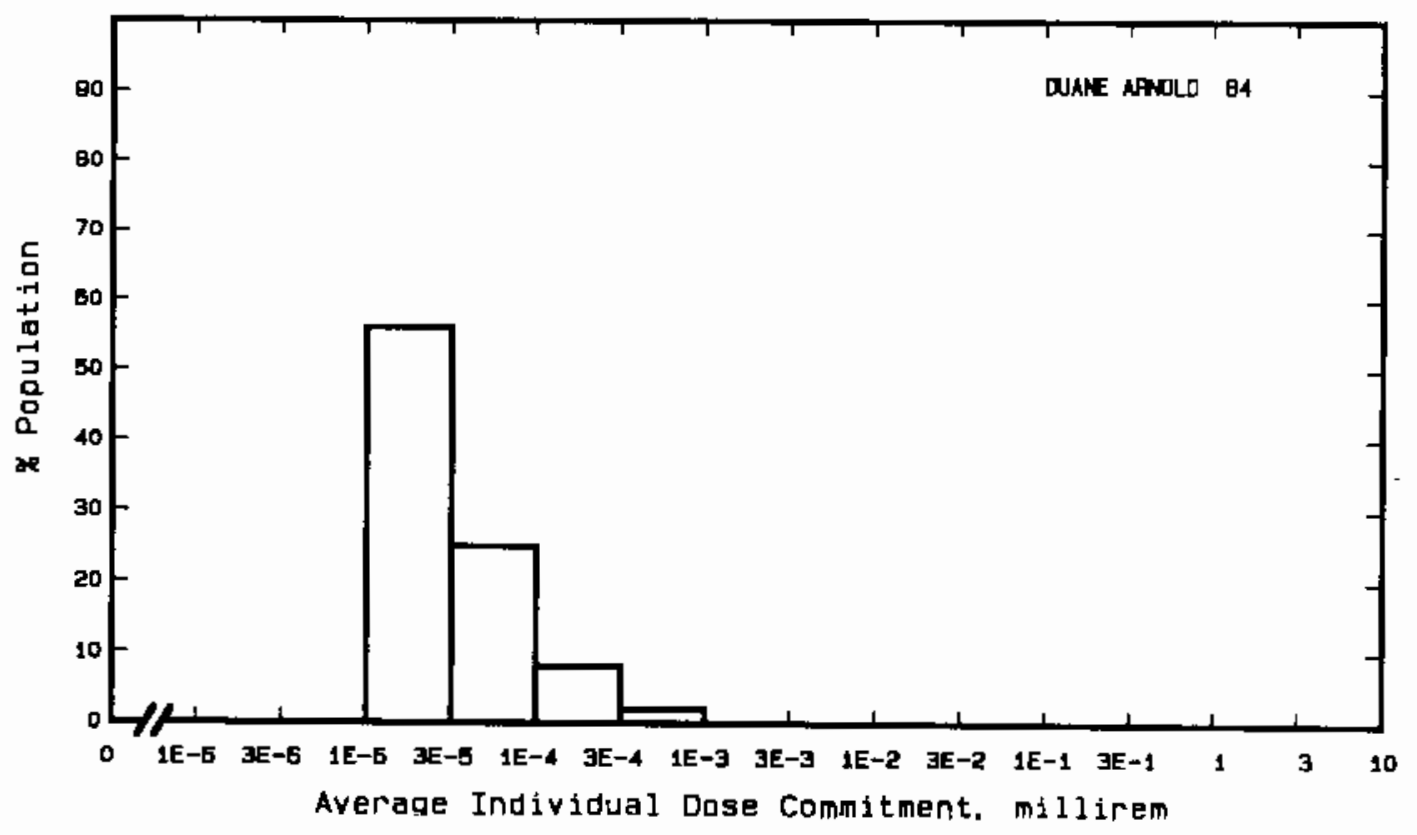


Site: J. M. FARLEY

DOTHAN, ALABAMA

Location: N $31.2228^{\circ}$ W $85.1126^{\circ}$

POPULATION DATA

Total Population Within 2-to-80-km Region: 3.6E5

Major Metropolitan Centers Within Region:

\begin{tabular}{lccc}
\multicolumn{1}{c}{ Center } & Population & \multicolumn{2}{c}{ Location } \\
Dothan & 49,000 & & \\
Enterprise & 18,000 & $27 \mathrm{~km}$ & W \\
Ozark & 13,000 & $71 \mathrm{~km}$ & W \\
Eufaula & 12,000 & $56 \mathrm{~km}$ & WNW \\
Bainbridge & 11,000 & $75 \mathrm{~km}$ & $\mathrm{~N}$ \\
& & $62 \mathrm{~km}$ & SE
\end{tabular}

SITE-SPECIFIC DATA - AIRBORNE PATHWAYS

Average Annual State Production

of Crops and Animal Products

In 80-km Radius Circle

Regional Productivity Factor:

Animal Grazing Factor:

Meteorology Period of Record: 1 APR 71 - 31 MAR 75 Recovery: 100\%
Veg: $1.7 \mathrm{E} 7 \mathrm{kilogram}$

Mi Tk: $5.7 \mathrm{E} 7$ Titer

Meat: $8.6 \mathrm{E} 7 \mathrm{ki}$ logram

0.95

0.8

SITE-SPECIFIC DATA - WATERBORNE PATHWAYS via CHATTAHOOCHEE RIVER

Average River Flow

at Site: $12,000 \mathrm{ft}^{3} / \mathrm{s}$

Drinking Water:

Exposed Population: None

Fish:

Edible Harvest: $2.3 \mathrm{E} 5 \mathrm{~kg} / \mathrm{yr}$

Dilution Factor: 1 
POPULATION DOSE-COMMITMENT ESTIMATES ANO

AVERAGE INOIVIOUAL OOSE-COMMITMENT HISTOGRAM FOR

J. M. FARLEY 1 AND 2

Dose Commitments (person-rem) from Waterborne Pathways

Total Body GI-LLI $\underline{\text { Thyroid }}$ Bone Liver

$\begin{array}{llllll}\text { Infant } & 0.0 \mathrm{E}+00 & 0.0 \mathrm{E}+00 & 0.0 \mathrm{E}+00 & 0.0 \mathrm{E}+00 & 0.0 \mathrm{E}+00 \\ \text { Child } & 2.9 \mathrm{E}-03 & 4.6 \mathrm{E}-03 & 5.3 \mathrm{E}-04 & 1.3 \mathrm{E}-02 & 1.5 \mathrm{E}-02 \\ \text { Teen } & 5.4 \mathrm{E}-03 & 9.4 \mathrm{E}-\mathrm{D3} & 4.1 \mathrm{E}-04 & 7.9 \mathrm{E}-03 & 1.3 \mathrm{E}-02 \\ \text { AduTt } & 5.7 \mathrm{E}-02 & 8.1 \mathrm{E}-02 & 2.9 \mathrm{E}-03 & 4.6 \mathrm{E}-02 & 7.8 \mathrm{E}-02 \\ \text { TOTAL } & 6.6 \mathrm{E}-02 & 9.5 \mathrm{E}-02 & 3.9 \mathrm{E}-03 & 6.7 \mathrm{E}-02 & 1.1 \mathrm{E}-01\end{array}$

Dose Commitments (person-rem) from Airborne Pathways

Total Body GI-LLI Thyroid Bone Liver Lung

$\begin{array}{lllllll}\text { Infant } & 2.2 \mathrm{E}-03 & 2.2 \mathrm{E}-03 & 4.2 \mathrm{E}-03 & 1.6 \mathrm{E}-03 & 2.2 \mathrm{E}-03 & 2.3 \mathrm{E}-03 \\ \text { Child } & 2.7 \mathrm{E}-02 & 2.7 \mathrm{E}-02 & 3.9 \mathrm{E}-02 & 1.7 \mathrm{E}-02 & 2.7 \mathrm{E}-02 & 2.9 \mathrm{E}-02 \\ \text { Teen } & 1.9 \mathrm{E}-02 & 1.9 \mathrm{E}-02 & 2.4 \mathrm{E}-02 & 1.3 \mathrm{E}-02 & 1.9 \mathrm{E}-02 & 2.1 \mathrm{E}-02 \\ \text { Adult } & 1.1 \mathrm{E}-01 & 1.1 \mathrm{E}-01 & 1.3 \mathrm{E}-01 & 7.7 \mathrm{E}-02 & 1.1 \mathrm{E}-01 & 1.2 \mathrm{E}-01 \\ \text { TOTAL } & 1.6 \mathrm{E}-\mathrm{D1} & 1.6 \mathrm{E}-01 & 1.9 \mathrm{E}-01 & 1.1 \mathrm{E}-01 & 1.6 \mathrm{E}-01 & 1.7 \mathrm{E}-01\end{array}$

Production/Consumption factors:

Produce: <1 Milk: 1.2 Meat: 2.8

Fraction of Population Receiving an Average Individual Total-Body Dose Commitment from Airborne Pathways

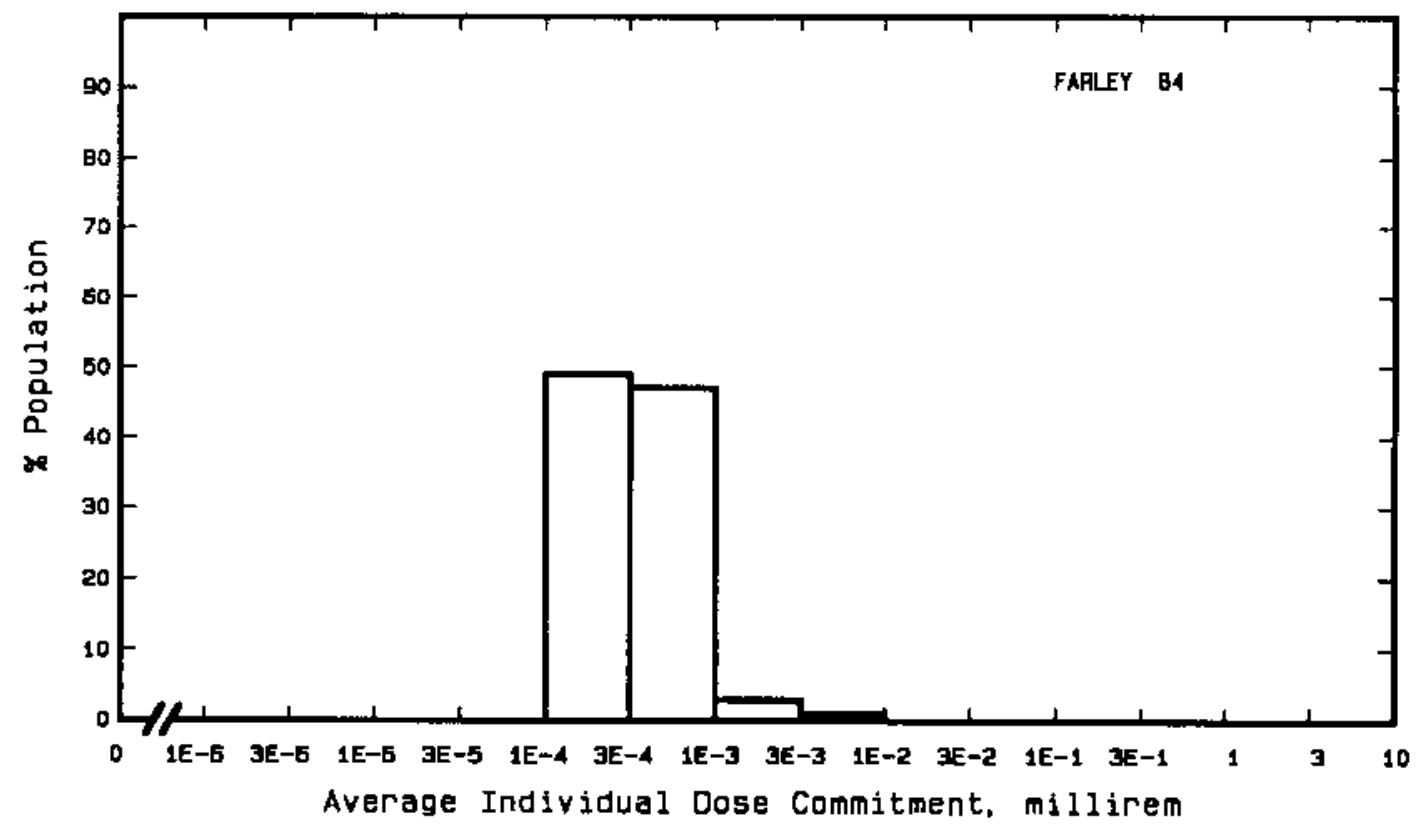


Site: J. A. FITZPATRICK

OSWEGO, NEW YORK

Location: N $43.5239^{\circ} \quad$ W $76.3983^{\circ}$

POPULATION DATA

Total Population Within 2-to-80-km Region: $8.5 E 5$

Major Metropolitan Centers Within Region:

\begin{tabular}{|c|c|c|c|}
\hline Center & Population & \multicolumn{2}{|c|}{ Location } \\
\hline Syracuse SMSA & 640,000 & $56 \mathrm{~km}$ & SSE \\
\hline Rome & 48,000 & $80 \mathrm{~km}$ & ESE \\
\hline Auburn & 33,000 & $66 \mathrm{~km}$ & SSW \\
\hline Watertown & 28,000 & $64 \mathrm{~km}$ & $\mathrm{NE}$ \\
\hline Kingston & 24,000 & $79 \mathrm{~km}$ & $N$ \\
\hline
\end{tabular}

SITE-SPECIFIC DATA - AIRBORNE PATHWAYS

Average Annual State Production

of Crops and Animal Products

In 80-km Radius Circle

Regional Productivity Factor:

Animal Grazing Factor:

Meteorology Period of Record: 1 JAN 74 - 31 DEC 75 Recovery: 97\%
Veg: $7.6 E 7$ kilogram

MiTk: $7.0 \mathrm{E} 8$ liter

Meat: $3.3 \mathrm{E} 7 \mathrm{kilogram}$

0.7

0.5

SITE-SPECIFIC DATA - WATERBORNE PATHWAYS via LAKE ONTARIO

\begin{tabular}{|c|c|}
\hline & $\begin{array}{l}\text { Average Dilution Flow } \\
\text { from Plant: } 830 \mathrm{ft}^{3} / \mathrm{s}\end{array}$ \\
\hline Drinking Water: & $\begin{array}{l}\text { Exposed Population: } 53 \rho_{\mathrm{B}} 90 \mathrm{O}^{(\mathrm{a})} \\
\text { Dilution Factor: } 0.003\end{array}$ \\
\hline Fish: & $\begin{array}{l}\text { Edible Harvest: } 7.3 \mathrm{E} 5 \mathrm{~kg} / \mathrm{yr} \\
\text { Dilution Factor: } 0.005\end{array}$ \\
\hline
\end{tabular}

(a) Population exposed to drinking water derived from Nine Mile Point FES (1974).

(b) DiTution factors derived from FES (1973). 
POPULATION DOSE-COMMITMENT ESTIMATES ANO

AVERAGE INDIVIDUAL DOSE-COMMITMENT HISTOGRAM FOR

$$
\text { J. A. FITZPATRICK }
$$

Dose Commitments (person-rem) from Waterborne Pathways

$$
\text { Total Body GI-LLI Thyroid Bone Liver }
$$

$\begin{array}{llllll}\text { Infant } & 1.4 \mathrm{E}-05 & 1.5 \mathrm{E}-05 & 1.2 \mathrm{E}-05 & 3.3 \mathrm{E}-06 & 1.5 \mathrm{E}-05 \\ \text { Child } & 2.2 \mathrm{E}-04 & 2.6 \mathrm{E}-04 & 1.2 \mathrm{E}-04 & 2.5 \mathrm{E}-04 & 4.1 \mathrm{E}-04 \\ \text { Teen } & 1.5 \mathrm{E}-04 & 2.5 \mathrm{E}-04 & 4.2 \mathrm{E}-05 & 1.4 \mathrm{E}-04 & 2.8 \mathrm{E}-04 \\ \text { AduTt } & 1.3 \mathrm{E}-03 & 2.3 \mathrm{E}-03 & 3.5 \mathrm{E}-04 & 8.0 \mathrm{E}-04 & 1 . \mathrm{BE}-03 \\ \text { TOTAL } & 1.7 \mathrm{E}-03 & 2.8 \mathrm{E}-03 & 5.2 \mathrm{E}-04 & 1.2 \mathrm{E}-03 & 2.5 \mathrm{E}-03\end{array}$

Dose Commitments (person-rem) from Airborne Pathways

\begin{tabular}{lccccccc} 
& Total Body & GI-LLI & Thyroid & Bone & Liver & Lung \\
\cline { 2 - 2 } Infant & $1.4 \mathrm{E}-02$ & $1.4 \mathrm{E}-02$ & & $7.5 \mathrm{E}-02$ & $1.5 \mathrm{E}-02$ & $1.4 \mathrm{E}-02$ & $1.4 \mathrm{E}-02$ \\
Child & $1.6 \mathrm{E}-01$ & $1.6 \mathrm{E}-01$ & $5.6 \mathrm{E}-01$ & $2.1 \mathrm{E}-01$ & $1.6 \mathrm{E}+01$ & $1.6 \mathrm{E}-01$ \\
Teen & $1.1 \mathrm{E}-01$ & $1.1 \mathrm{E}-01$ & $2.7 \mathrm{E}-01$ & $1.3 \mathrm{E}-01$ & $1.1 \mathrm{E}-01$ & $1.2 \mathrm{E}-01$ \\
Aduit & $6.9 \mathrm{E}-01$ & $6.9 \mathrm{E}-01$ & $1.2 \mathrm{E}-00$ & $7.4 \mathrm{E}-01$ & $6.8 \mathrm{E}-01$ & $7.1 \mathrm{E}+01$ \\
TOTAL & $9.7 \mathrm{E}-01$ & $9.8 \mathrm{E}-01$ & $2.1 \mathrm{E}+00$ & $1.1 \mathrm{E}+00$ & $9.7 \mathrm{E}-01$ & $1.0 \mathrm{E}+00$
\end{tabular}

Production/Consumption factors:

Produce: <1 Milk: $4.4 \quad$ Meat: <1

Fraction of Population Receiving an Average Individual

Total-Body Dose Commitment from Airborne Pathways

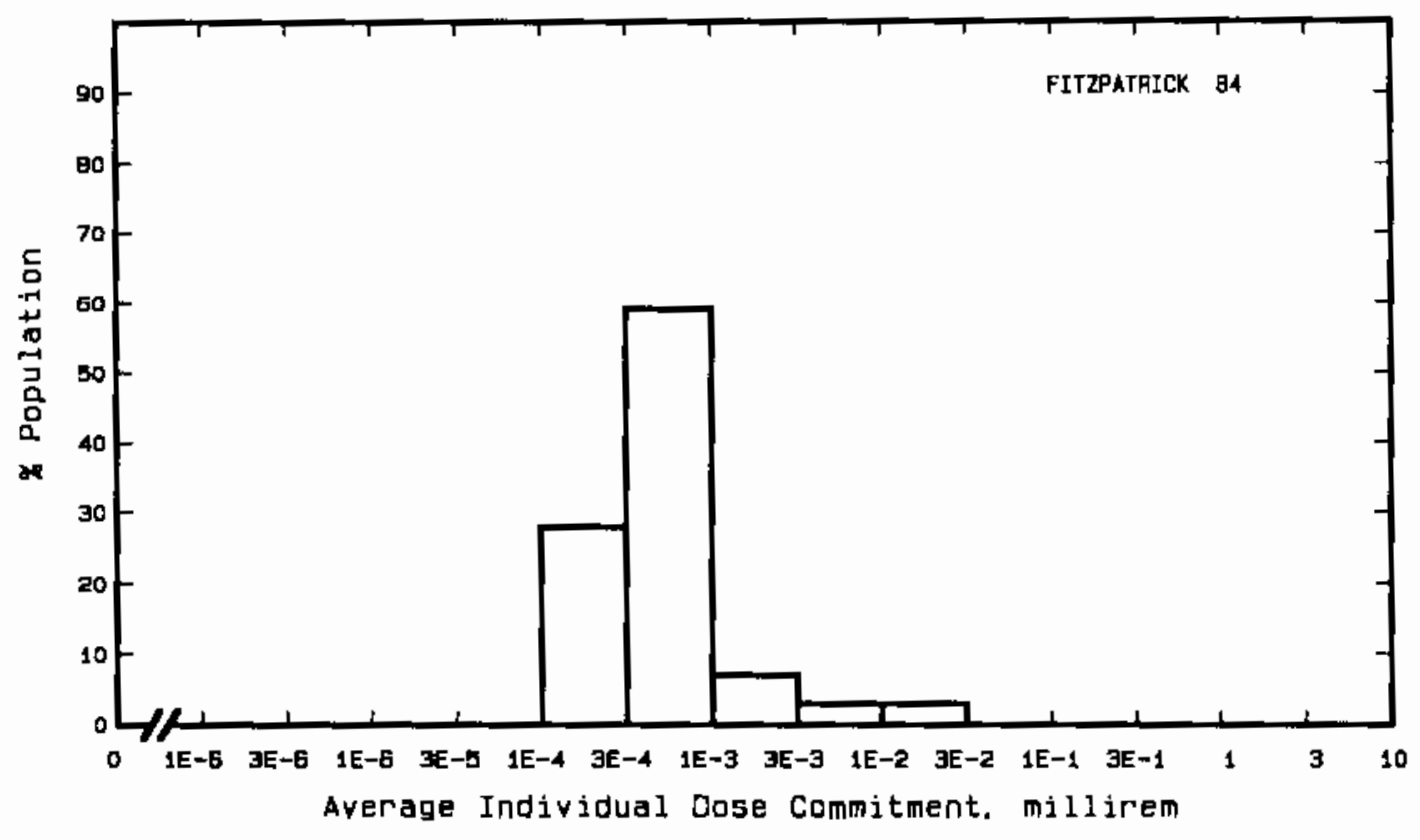


Site: FORT CALHOUN

WASHINGTON COUNTY, NEBRASKA

Location: $\quad \mathrm{N} 41.5208^{\circ} \quad W 96.0767^{\circ}$

POPULATION DATA

Total Population Within 2-to-80-km Region: 7.6E5

Major Metropolitan Centers Within Region:

\begin{tabular}{lrrrr}
\multicolumn{1}{c}{ Center } & Population & & \multicolumn{2}{c}{ Location } \\
\cline { 2 - 3 } & & & & \\
Omaha & 580,000 & & $\mathrm{~km}$ & SSE \\
CounciT BTuffs & 56,000 & $34 \mathrm{~km}$ & SE \\
Freemont & 24,000 & $36 \mathrm{~km}$ & WSW \\
Bellevue & 22,000 & $44 \mathrm{~km}$ & SSE
\end{tabular}

SITE-SPECIFIC DATA - AIRBORNE PATHWAYS

Average Annual State Production

of Crops and Animal Products

In 80-km Radius Circle

Regional Productivity Factor:

Animal Grazing Factor:

Meteorology Period of Record: 1 JAN 74 - 31 DEC 74 Recovery: 98\%
Veg: $9.7 E 7$ kilogram

Mi Tk: 7.227 liter

Meat: $2.0 \mathrm{E} 8$ kilogram

1

0.5

SITE-SPECIFIC DATA - WATERBORNE PATHWAYS via MISSOURI RIVER

Average River Flow at Site: $27,000 \mathrm{ft}^{3} / \mathrm{s}$

Drinking Water:

Exposed Poputation: 580,000 (a)

Dilution Factor: I

Fish:

Edible Harvest: $1.0 \mathrm{E} 4 \mathrm{~kg} / \mathrm{yr}$ Dilution Factor: 1

(a) Drinking water poputation assumed to be Omaha SMSA (FES 1972). 
POPULATION DOSE-COMMITMENT ESTIMATES AND

AVERAGE INDIVIDUAL DOSE-COMMITMENT HISTOGRAM FOR

FORT CALHOUN

Dose Commitments (person-rem) from Waterborne Pathways

\begin{tabular}{|c|c|c|c|c|c|}
\hline & Total Body & GI-LLI & Thyroid & Bone & Liver \\
\hline $\begin{array}{l}\text { Infant } \\
\text { Child } \\
\text { Teen } \\
\text { Adult }\end{array}$ & $\begin{array}{l}1.2 \mathrm{E}-\mathrm{D} 2 \\
1.9 \mathrm{E}-01 \\
1.4 \mathrm{E}-01 \\
1.6 \mathrm{E}+00\end{array}$ & $\begin{array}{l}5.1 \mathrm{E}-03 \\
6.3 \mathrm{E}-02 \\
3.2 \mathrm{E}-02 \\
2.8 \mathrm{E}-01\end{array}$ & $\begin{array}{l}3.6 \mathrm{E}-02 \\
2.7 \mathrm{E}-01 \\
8.6 \mathrm{E}-02 \\
6.3 \mathrm{E}-01\end{array}$ & $\begin{array}{l}5.7 \mathrm{E}-02 \\
6.3 \mathrm{E}-01 \\
1.7 \mathrm{E}-01 \\
1.1 \mathrm{E}-00\end{array}$ & $\begin{array}{l}8.6 \mathrm{E}-02 \\
8.2 \mathrm{E}-01 \\
3.2 \mathrm{E}-01 \\
2.0 \mathrm{E}-00\end{array}$ \\
\hline TTAL & $1.9 \mathrm{E}+00$ & $3.8 \mathrm{E}-01$ & 1. $.0 E+00$ & $1.9 E+00$ & $3.3 E+00$ \\
\hline
\end{tabular}

Dose Commitments (person-rem) from Airborne Pathways

Total Body GI-LLI Thyroid Bone Liver Lung

$\begin{array}{lllllll}\text { Infant } & 2.3 \mathrm{E}-04 & 2.3 \mathrm{E}-04 & 4.7 \mathrm{E}-03 & 2.3 \mathrm{E}-04 & 2.4 \mathrm{E}-04 & 2.5 \mathrm{E}-04 \\ \text { ChiTd } & 2.7 \mathrm{E}-03 & 2.7 \mathrm{E}-03 & 4.7 \mathrm{E}-02 & 2.6 \mathrm{E}-03 & 2.9 \mathrm{E}-03 & 2.9 \mathrm{E}-03 \\ \text { Teen } & 1.9 \mathrm{E}-03 & 1.9 \mathrm{E}-03 & 1.9 \mathrm{E}-02 & 1.8 \mathrm{E}-03 & 2.0 \mathrm{E}-03 & 2.3 \mathrm{E}-03 \\ \text { AduTt } & 1.2 \mathrm{E}-02 & 1.1 \mathrm{E}-03 & 7.3 \mathrm{E}-02 & 1.1 \mathrm{E}-02 & 1.2 \mathrm{E}-02 & 1.3 \mathrm{E}-02 \\ \text { TOTAL } & 1.7 \mathrm{E}-02 & 1.6 \mathrm{E}-02 & 1.4 \mathrm{E}-01 & 1.5 \mathrm{E}-02 & 1.7 \mathrm{E}-02 & 1.8 \mathrm{E}-02\end{array}$

Production/Consumption factors:

Produce: < Milk: <1 Meat: 3.2

Fraction of Population Receiving an Average Individual

Total-Body Dose Commitment from Airborne Pathways

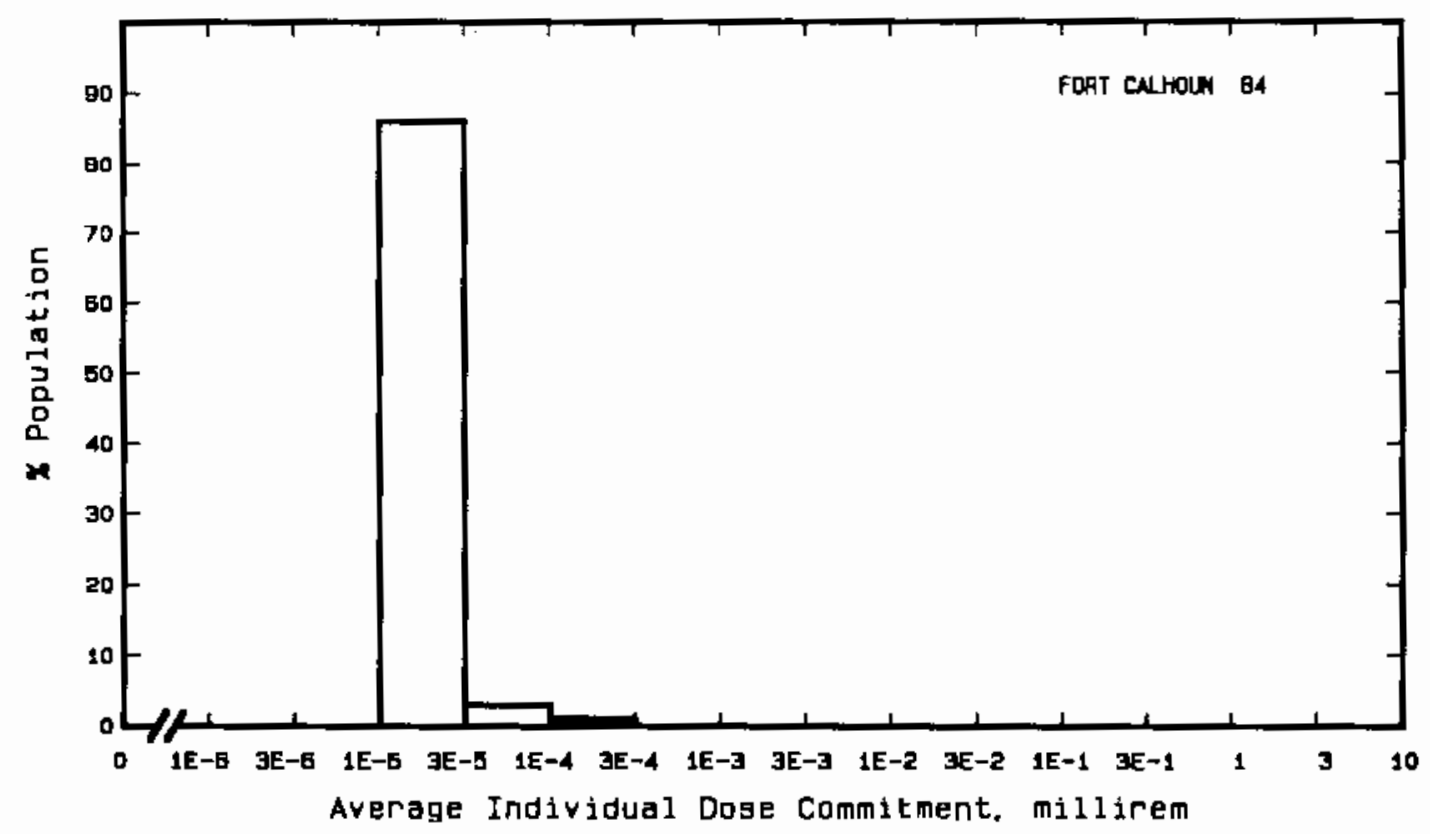


Site: R. E. GINNA

ONTARIO, NEW YORK

Location: $\quad$ N $43.2778^{\circ} \quad$ W $77.3089^{\circ}$

POPULATION DATA

Total Population Within 2-to-80-km Region: 1.2E6

Major Metropolitan Centers W'thin Region:

\begin{tabular}{lrrrr}
\multicolumn{1}{c}{ Center } & Population & \multicolumn{2}{c}{ Location } \\
\cline { 2 - 4 } & & & \\
Rochester SMSA & 970,000 & $27 \mathrm{~km}$ & WSW \\
Auburn & 32,000 & $71 \mathrm{~km}$ & ESE \\
Oswego & 20,000 & $67 \mathrm{~km}$ & ENE \\
Batavia & 17,000 & $78 \mathrm{~km}$ & WSW \\
Geneva & 15,000 & $52 \mathrm{~km}$ & SSE
\end{tabular}

SITE-SPECIFIC DATA - AIRBORNE PATHWAYS

Average Annual State Production

of Crops and Animal Products

In 80-km Radius Circle

Regional Productivity Factor:

Animal Grazing Factor:

Meteorology Period of Record: 1 JAN 66 - 31 DEC 67 Recovery: 89\%
Veg: $7.6 \mathrm{E} 7 \mathrm{ki}$ logram

Milk: $7.0 \mathrm{E} 8$ liter

Meat: $3.3 E 7$ kilogram

0.6

0.5

SITE-SPECIFIC DATA - WATERBORNE PATHWAYS via LAKE ONTARIO

Average Dilution Flow

from Plant: $340 \mathrm{ft}^{3} / \mathrm{s}$

Drinking Water:

Exposed Poputation: 560,000

Dilution Factor: 0.01

Fish:

Edible Harvest: $7.3 \mathrm{E} 5(\mathrm{~kg} / \mathrm{yr}$
DiTution Factor: 0.01

(a) Dilution factors from FES (1973). 
POPULATION DOSE-COMMITMENT ESTIMATES AND

AVERAGE INDIVIDUAL DOSE-COMMITMENT HISTOGRAM FOR

$$
\text { R. E. GINNA }
$$

Dose Commitments (person-rem) from Waterborne Pathways

\begin{tabular}{|c|c|c|c|c|c|}
\hline & Total Body & GI-LLI & Thyroid & Bone & Liver \\
\hline $\begin{array}{l}\text { Infant } \\
\text { Child } \\
\text { Teen } \\
\text { Adult }\end{array}$ & $\begin{array}{l}6.7 \mathrm{E}-03 \\
8.8 \mathrm{E}-02 \\
5.3 \mathrm{E}-02 \\
5.2 \mathrm{E}-01\end{array}$ & $\begin{array}{l}6.5 \mathrm{E}-\mathrm{D} 3 \\
7.5 \mathrm{E}-02 \\
3.0 \mathrm{E}-02 \\
2.6 \mathrm{E}-0 \mathrm{I}\end{array}$ & $\begin{array}{l}6.5 \mathrm{E}-03 \\
7.3 \mathrm{E}-02 \\
2.8 \mathrm{E}-02 \\
2.4 \mathrm{E}-01\end{array}$ & $\begin{array}{l}1.7 \mathrm{E}-03 \\
7.5 \mathrm{E}-02 \\
3.9 \mathrm{E}-02 \\
2.3 \mathrm{E}-01\end{array}$ & $\begin{array}{l}8.5 \mathrm{E}-03 \\
1.6 \mathrm{E}-01 \\
9.1 \mathrm{E}-02 \\
6.2 \mathrm{E}-01\end{array}$ \\
\hline TOTAL & $6.6 E-01$ & $3.7 \mathrm{E}-01$ & $3.4 \mathrm{E}-01$ & $3.4 \mathrm{E}-01$ & $8.8 E-01$ \\
\hline
\end{tabular}

Dose Comitments (person-rem) from Airborne Pathways

Total Body GI-LLI Thyroid Bone Liver Lung

$\begin{array}{lllllll}\text { Infant } & 4.4 \mathrm{E}-04 & 4.4 \mathrm{E}-04 & 1.4 \mathrm{E}-03 & 7.4 \mathrm{E}-05 & 4.4 \mathrm{E}-04 & 4.4 \mathrm{E}-04 \\ \text { Child } & 5.8 \mathrm{E}-03 & 5.8 \mathrm{E}-03 & 1.1 \mathrm{E}-02 & 8.2 \mathrm{E}-04 & 5.8 \mathrm{E}-03 & 5.8 \mathrm{E}-03 \\ \text { Teen } & 3.6 \mathrm{E}-03 & 3.6 \mathrm{E}-03 & 5.8 \mathrm{E}-03 & 5.9 \mathrm{E}-04 & 3.6 \mathrm{E}-03 & 3.7 \mathrm{E}-03 \\ \text { Adult } & 1.9 \mathrm{E}-02 & 1.9 \mathrm{E}-02 & 2.6 \mathrm{E}-02 & 3.6 \mathrm{E}-03 & 1.9 \mathrm{E}-02 & 2.0 \mathrm{E}-02 \\ \text { T0TAL } & 2.9 \mathrm{E}-02 & 2.9 \mathrm{E}-02 & 4.5 \mathrm{E}-02 & 5.1 \mathrm{E}-03 & 2.9 \mathrm{E}-02 & 3.0 \mathrm{E}-02\end{array}$

Production/Consumption factors:

Produce: <I Milk: 2.7 Meat: <1

Fraction of Population Receiving an Average Individual Total-Body oose Commitment from Airborne Pathways

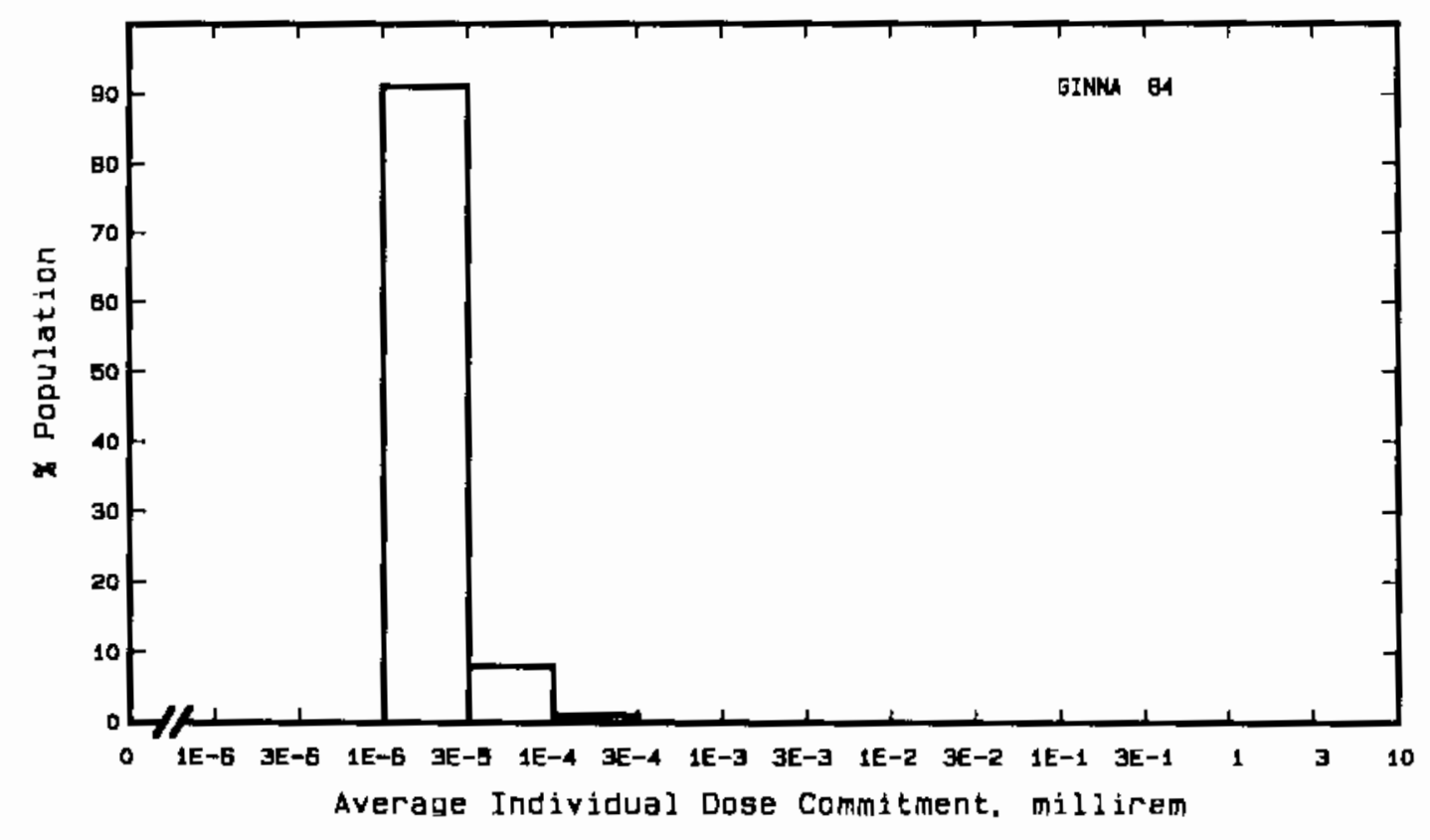


Site: GRAND GULF

PORT GIBSON, MISSISSIPPI

Location: $\quad \mathrm{N} 32.0270^{\circ} \quad W 91.2530^{\circ}$

POPULATION DATA

Total Population Within 2-to-80-km Region: 3.2E6

Major Metropolitan Centers Within Region:

\begin{tabular}{lcccc} 
Center & Population & \multicolumn{2}{c}{ Location } \\
\cline { 2 - 4 } & 26,000 & & & \\
Vicks & 9,700 & $40 \mathrm{~km}$ & NNE \\
Tallutah & 20,000 & $45 \mathrm{~km}$ & NNW \\
Natches & 11,000 & $60 \mathrm{~km}$ & SSW \\
Brookhaven & & $76 \mathrm{~km}$ & SE
\end{tabular}

SITE-SPECIFIC OATA - AIRBORNE PATHWAYS

Average Annual State Production

of Crops and Animal Products

In 80-km Radius Circle

Regional Productivity Factor:

Animal Grazing Factor:

Meteorology Period of Record: I AUG 72 - 31 JUL 73 Recovery: 99\%
Veg: $4.4 \mathrm{E} 6$ ki logram

Milk: $7.1 \mathrm{E} 7$ liter

Meat: $9.9 E 7$ kilogram

0.9

0.8

SITE-SPECIFIC OATA - WATERBORNE PATHWAYS via MISSISSIPPI RIVER

Average River

Flow at Site: $430,000 \mathrm{ft}^{3} / \mathrm{s}$

Orinking Water:

Exposed Population: None

Fish:

Edible Harvest: $7.0 \mathrm{E} 5 \mathrm{~kg} / \mathrm{yr}$

Dilution Factor: 1

Invertebrates:

Edible Harvest: (a)

Dilution Factor:

(a) Not considered. 


\section{POPULATION DOSE-COMMITMENT ESTIMATES AND \\ AVERAGE INDIVIDUAL DOSE-COMMITMENT HISTOGRAM FOR \\ GRAND GULF}

Dose Commitments (person-rem) from Waterborne Pathways

Total Body GI-LLI $\underline{\text { Thyroid }}$ Bone Liver

$\begin{array}{llllll}\text { Infant } & 0.0 \mathrm{E}+00 & 0.0 \mathrm{E}+00 & 0.0 \mathrm{E}+00 & 0.0 \mathrm{E}+00 & 0.0 \mathrm{E}+00 \\ \text { Child } & 8.0 \mathrm{E}-07 & 1.5 \mathrm{E}-06 & 3.9 \mathrm{E}-08 & 1.7 \mathrm{E}-06 & 1.8 \mathrm{E}-06 \\ \text { Teen } & 5.3 \mathrm{E}-07 & 3.3 \mathrm{E}-06 & 3.1 \mathrm{E}-08 & 9.6 \mathrm{E}-07 & 1.5 \mathrm{E}-06 \\ \text { Adu1t } & 3.2 \mathrm{E}-06 & 2.9 \mathrm{E}-05 & 2.2 \mathrm{E}-07 & 5.6 \mathrm{E}-06 & 9.0 \mathrm{E}-06 \\ \text { TOTAL } & 4.5 \mathrm{E}-06 & 3.4 \mathrm{E}-05 & 2.9 \mathrm{E}-07 & 8.3 \mathrm{E}-06 & 1.2 \mathrm{E}-05\end{array}$

Dose Commitments (person-rem) from Airborne Pathways

Total Body GI-LLI Thyroid sone Liver Lung

$\begin{array}{lllllll}\text { Infant } & 2.8 \mathrm{E}-05 & 2.8 \mathrm{E}-05 & 2.8 \mathrm{E}-05 & 2.7 \mathrm{E}-05 & 2.8 \mathrm{E}-05 & 3.0 \mathrm{E}-05 \\ \text { Child } & 3.1 \mathrm{E}-04 & 4.4 \mathrm{E}-04 & 3.1 \mathrm{E}-04 & 3.1 \mathrm{E}-04 & 3.1 \mathrm{E}-04 & 3.3 \mathrm{E}-04 \\ \text { Teen } & 2.2 \mathrm{E}-04 & 3.8 \mathrm{E}-04 & 2.3 \mathrm{E}-04 & 2.3 \mathrm{E}-04 & 2.2 \mathrm{E}-04 & 2.5 \mathrm{E}-04 \\ \text { AduTt } & 1.4 \mathrm{E}-03 & 2.8 \mathrm{E}-03 & 1.4 \mathrm{E}-03 & 1.4 \mathrm{E}-03 & 1.4 \mathrm{E}-03 & 1.4 \mathrm{E}-03 \\ \text { TOTAL } & 1.9 \mathrm{E}-03 & 3.7 \mathrm{E}-03 & 1.9 \mathrm{E}-03 & 1.9 \mathrm{E}-03 & 1.9 \mathrm{E}-03 & 2.1 \mathrm{E}-03\end{array}$

Production/Consumption factors:

Produce: <1 Milk: 1.5 Meat: 3.4

Fraction of Population Receiving an Average Individual Total-Body Dose Commitment from Airborne Pathways

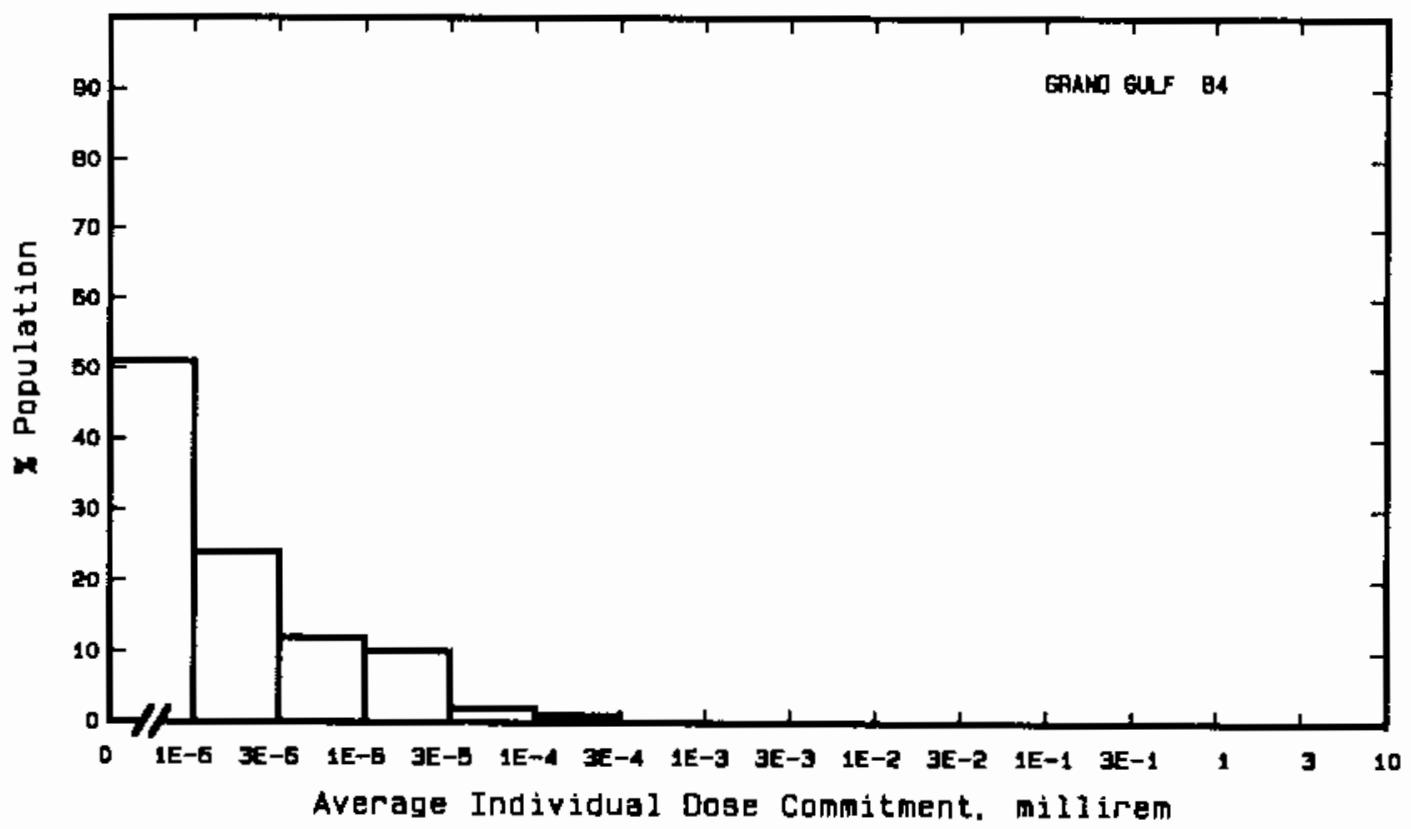


Site: HADDAM NECK

HADDAM NECK, CONNECTICUT

Location: N $41.4819^{\circ}$ W $72.4992^{\circ}$

POPULATIDN DATA

Total Population Within 2-to-80-km Region: 3.4E6

Major Metropolitan Centers Within Region:

Center

Hartford SMSA

Springfield-Chicopee-Holyoke SMSA

New Haven-West Haven SMSA

Bridgeport SMSA

New London-Norwich
Population

730,000

530,000

420,000

400,000

250,000
Location

$35 \mathrm{~km} \quad \mathrm{NNW}$

$70 \mathrm{~km} \mathrm{~N}$

$40 \mathrm{~km} \quad W S W$

$66 \mathrm{~km}$ WSW

$35 \mathrm{~km} \quad$ ESE

SITE-SPECIFIC DATA - AIRBORNE PATHWAYS

Average Annual State Production

of Crops and Animal Products

In 80-km Radius Circle

Regional Productivity Factor:

Animal Grazing Factor:
Veg: $3.2 \mathrm{E7} \mathrm{kilogram}$

Milk: 4.4E8 1iter

Meat: 2.DE7 kiTogram

0.7

0.6

Meteorology Period of Record: I JAN $75-31$ DEC 75 Recovery: 95\%

SITE-SPECIFIC DATA - WATERBORNE PATHWAYS via DISCHARGE CANAL TO CONN. RIVER

Average Discharge Canal

Flow at Site: $710 \mathrm{ft}^{3} / \mathrm{s}$

Drinking Water:

Exposed Poputation: None

Fish:

Edible Harvest: $9.1 \mathrm{E3}^{(\mathrm{a})} \mathrm{kg} / \mathrm{yr}$

DiTution Factor: 1

(a) Caught in discharge canal according to FES (1973). 
POPULATION OOSE-COMMITMENT ESTIMATES AND

AVERAGE INDIVIDUAL DOSE-COMMITMENT HISTOGRAM FOR

HADOAM NECK

Dose Commitments (person-rem) from Waterborne Pathways

Total Body GI-LLI Thyroid Bone Liver

$\begin{array}{llllll}\text { Infant } & 0.0 \mathrm{E}+00 & 0.0 \mathrm{E}+00 & 0.0 \mathrm{E}+00 & 0.0 \mathrm{E}+00 & 0.0 \mathrm{E}+00 \\ \text { Child } & 1.3 \mathrm{E}-02 & 1.2 \mathrm{E}-03 & 9.1 \mathrm{E}-04 & 6.2 \mathrm{E}-02 & 6.8 \mathrm{E}-02 \\ \text { Teen } & 2.4 \mathrm{E}-02 & 1.8 \mathrm{E}-03 & 7.6 \mathrm{E}-04 & 3.8 \mathrm{E}-02 & 5.8 \mathrm{E}-02 \\ \text { Adult } & 2.6 \mathrm{E}-01 & 1.4 \mathrm{E}-02 & 5.7 \mathrm{E}-03 & 2.2 \mathrm{E}-01 & 3.4 \mathrm{E}-01 \\ \text { TOTAL } & 2.9 \mathrm{E}-01 & 1.7 \mathrm{E}-02 & 7.4 \mathrm{E}-03 & 3.2 \mathrm{E}-01 & 4.7 \mathrm{E}-01\end{array}$

Dose Commitments (person-rem) from Airborne Pathways

Total Body GI-LLI Thyroid Bone Liver Lung

$\begin{array}{lllllll}\text { Infant } & 1.7 \mathrm{E}-02 & \mathrm{I} .6 \mathrm{E}-02 & 1.1 \mathrm{E}-01 & \mathrm{I} .4 \mathrm{E}-02 & 1.7 \mathrm{E}-02 & 1.8 \mathrm{E}-02 \\ \text { Child } & 1.9 \mathrm{E}-01 & 1.9 \mathrm{E}-01 & 7.3 \mathrm{E}-01 & 1.5 \mathrm{E}-01 & 1.9 \mathrm{E}-01 & 2.1 \mathrm{E}-01 \\ \text { Teen } & 1.4 \mathrm{E}-01 & 1.4 \mathrm{E}-0 \mathrm{I} & 3.7 \mathrm{E}-01 & 1.1 \mathrm{E}-01 & 1.4 \mathrm{E}-01 & 1.6 \mathrm{E}-01 \\ \text { AduTt } & 8.1 \mathrm{E}-01 & 8.1 \mathrm{E}-01 & 1.7 \mathrm{E}-00 & 6.5 \mathrm{E}-01 & 8.1 \mathrm{E}-01 & 8.9 \mathrm{E}-01 \\ \text { TOTAL } & 1.2 \mathrm{E}+00 & 1.2 \mathrm{E}+00 & 2.9 \mathrm{E}+00 & 9.3 \mathrm{E}-01 & 1.2 \mathrm{E}+00 & 1.3 \mathrm{E}+00\end{array}$

Production/Consumption factors:

Produce: <1 Milk: $<1 \quad$ Meat: $<1$

Fraction of Population Receiving an Average Individual

Total-Body Dose Commitment from Airborne Pathways

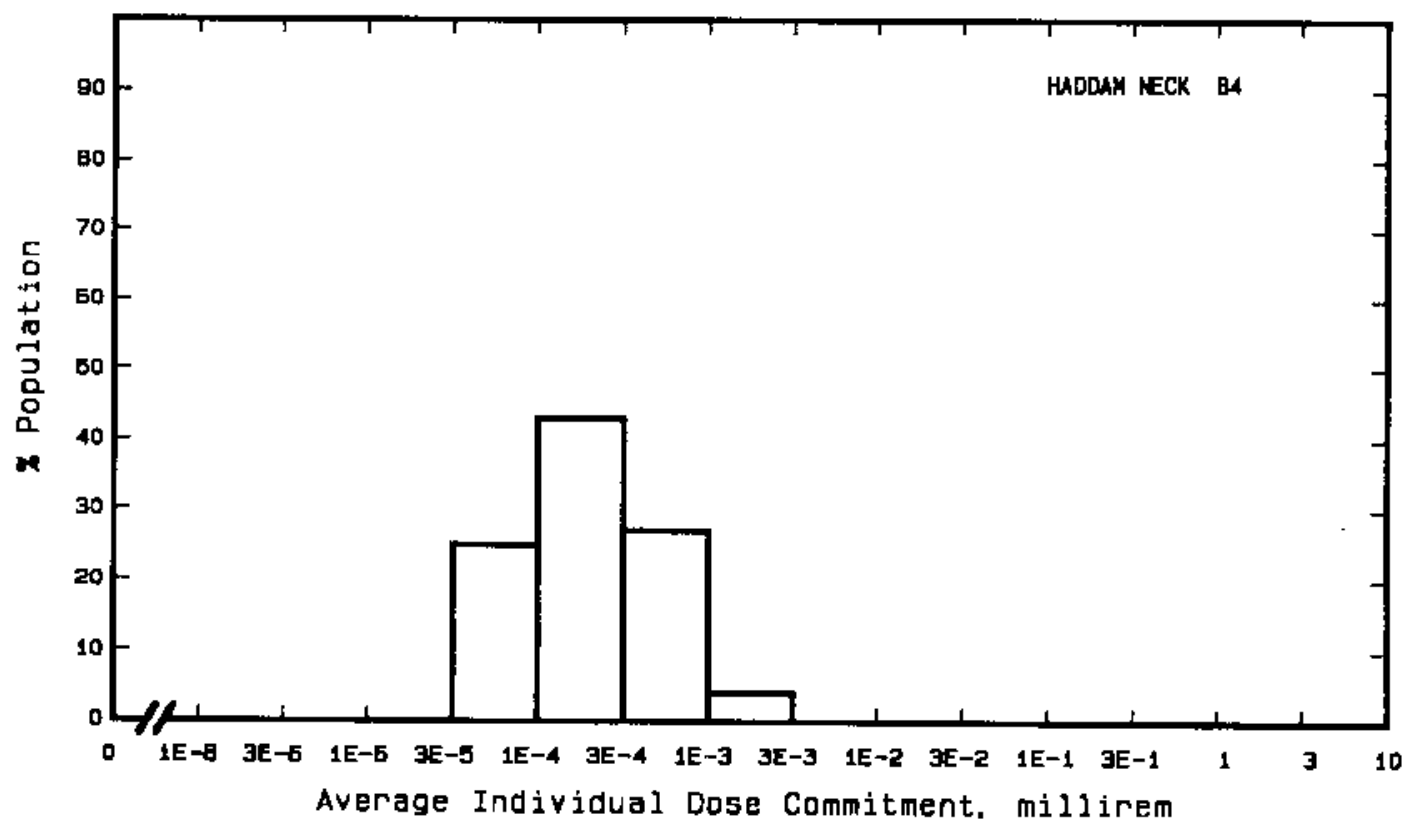


Site: E. I. HATCH

BAXLEY, GEORGIA

Location: $\quad N 31.9342^{\circ} \quad$ W $82.3444^{\circ}$

POPULATION DATA

Total Population Within 2-to-80-km Region: 3.2E5

Major Metropolitan Centers Within Region:

Center

Waycross

Statesboro

Hinesville

Douglas

Vidalia
Population

20,000

16,000

12,000

12,000

10,000
Location

$80 \mathrm{~km} \quad \mathrm{~S}$

$78 \mathrm{~km} \quad \mathrm{NE}$

$71 \mathrm{~km} \quad \mathrm{E}$

$67 \mathrm{~km} \quad \mathrm{SW}$

SITE-SPECIFIC DATA - AIRBORNE PATHWAYS

Average Annual State Production

of Crops and Animal Products

In 80-km Radius Circte

Regional Productivity Factor:

Animal Grazing Factor:

Meteorology Period of Record: 1 JUN 70 - 31 AUG 74 Recovery: 87\%
Veg: $8.8 \mathrm{E} 6$ kilogram

MiTk: 7.007 liter

Meat: $8.1 E 7$ kilogram

1

0.8

SITE-SPECIFIC DATA - WATERBORNE PATHWAYS via ALTAMAHA RIVER

Average River Flow at Site: $13,000 \mathrm{ft}^{3} / \mathrm{s}$

Drinking Water:

Exposed Population: None

Fish:

Edible Harrest: $6.3 \mathrm{E} 5^{(\mathrm{a})} \mathrm{kg} / \mathrm{yr}$ Dilution Factor: 1

(a) Commercial catch plus 3 pounds of game fish per year taken from river by average person according to FES (1972). 
POPULATION DOSE-COMMITMENT ESTIMATES AND

AVERAGE INDIVIDUAL DOSE-COMMITMENT HISTOGRAM FOR

E. I. HATCH 1 AND 2

Dose Commitments (person-rem) from Waterborne Pathways

\begin{tabular}{|c|c|c|c|c|}
\hline Tota 1 Body & GI-LLI & Thyroid & Bone & Liver \\
\hline $\begin{array}{l}0.0 E+00 \\
3.5 E-01 \\
6.5 E-01 \\
6.9 E+00\end{array}$ & $\begin{array}{l}0.0 \mathrm{E}+00 \\
2.1 \mathrm{E}-02 \\
4.5 \mathrm{E}-02 \\
3.9 \mathrm{E}-01\end{array}$ & $\begin{array}{l}0.0 \mathrm{E}+00 \\
8.3 \mathrm{E}-03 \\
6.0 \mathrm{E}-03 \\
3.9 \mathrm{E}-02\end{array}$ & $\begin{array}{l}0.0 \mathrm{E}+00 \\
1.5 \mathrm{E}+00 \\
9.3 \mathrm{E}-01 \\
5.4 \mathrm{E}+00\end{array}$ & $\begin{array}{l}0.0 E+00 \\
1.9 E+00 \\
1.6 E+00 \\
9.5 E+00\end{array}$ \\
\hline 7. $9 E+00$ & $4.5 \mathrm{E}-01$ & $5.3 E-02$ & $7.9 \mathrm{E}+00$ & 1. $3 E+01$ \\
\hline
\end{tabular}

Dose Commitments (person-rem) from Airborne Pathways

Total Body GI-LLI Thyroid Bone Liver Lung

$\begin{array}{lllllll}\text { Infant } & 2.2 \mathrm{E}-03 & 1.8 \mathrm{E}-03 & 2.8 \mathrm{E}-01 & 2.8 \mathrm{E}-03 & 3.1 \mathrm{E}-03 & 2.0 \mathrm{E}-03 \\ \text { Child } & 2.4 \mathrm{E}-02 & 2.1 \mathrm{E}-02 & 1.5 \mathrm{E}-00 & 2.6 \mathrm{E}-02 & 2.8 \mathrm{E}-02 & 2.2 \mathrm{E}-02 \\ \text { Teen } & 1.7 \mathrm{E}-02 & 1.5 \mathrm{E}-02 & 5.9 \mathrm{E}-01 & 1.6 \mathrm{E}-02 & 1.8 \mathrm{E}-02 & 1.7 \mathrm{E}-02 \\ \text { Adult } & 9.6 \mathrm{E}-02 & 9.1 \mathrm{E}-02 & 2.0 \mathrm{E}+00 & 9.1 \mathrm{E}-02 & 1.0 \mathrm{E}-01 & 9.5 \mathrm{E}-02 \\ \text { TOTAL } & 1.4 \mathrm{E}-01 & 1.3 \mathrm{E}-01 & 4.4 \mathrm{E}+00 & 1.4 \mathrm{E}-01 & 1.5 \mathrm{E}-01 & 1.4 \mathrm{E}-01\end{array}$

Production/Consumption factors:

Produce: <1 Milk: 1.7 Meat: 3.1

Fraction of Population Receiving an Average Individual Totel-Body Dose Commitment from Airborne Pathways

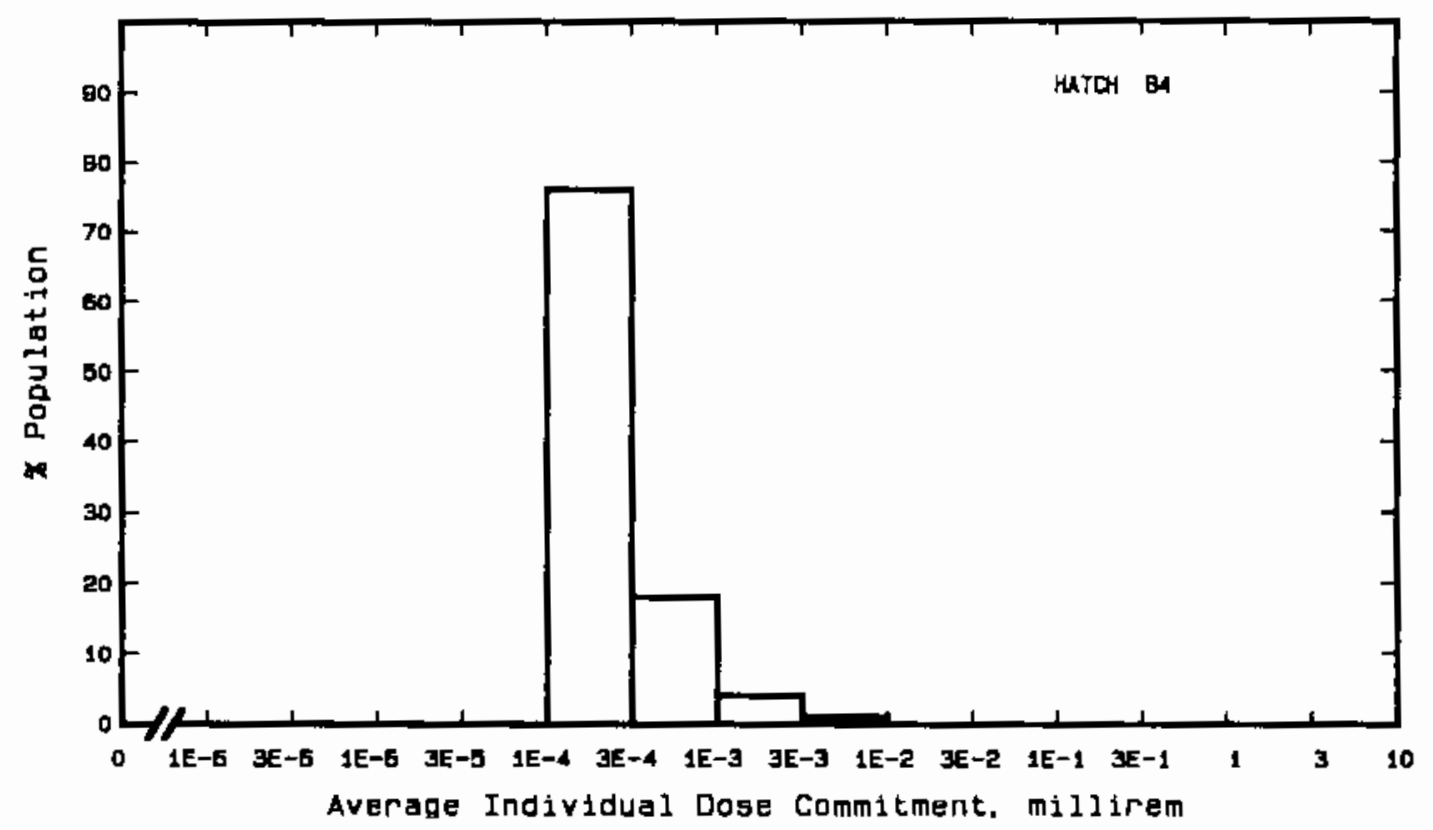


Site: INDIAN POINT

BUCHANAN, NEW YORK

Location: N $41.2714^{\circ}$ W $73.9525^{\circ}$

POPULATION DATA

Total Population Within 2-to-80-km Region: 1.5E7

Major Metropolitan Centers Within Region:

Center

New York SMSA

Newark SMSA

Nassau County SMSA

Jersey City SMSA

Paterson-C? ifton-Passaic SMSA
Population

$9,200,000$

$2,000,000$

$1,300,000$

560,000

450,000
Location

$57 \mathrm{~km} \quad \mathrm{~S}$

$62 \mathrm{~km}$ SSW

$70 \mathrm{~km} \quad$ SSE

$61 \mathrm{~km} \mathrm{~S}$

$44 \mathrm{~km} \quad \mathrm{SSW}$

SITE-SPECIFIC DATA - AIRBORNE PATHWAYS

Average Annua T State Production

of Crops and Anima 1 Products

In 80-km Radius Circle

Regional Productivity Factor:

Animal Grazing Factor:
Veg: $7.6 \mathrm{E} 7$ kilogram

Milk: 7.0E8 Titer

Meat: $3.3 \mathrm{E} 7$ kilogram

0.8

0.5

Meteorology Period of Record: I JAN 75 - 31 DEC 75 Recovery: 96\%

SITE-SPECIFIC DATA - WATERBORNE PATHWAYS via HUDSON RIVER

Average River Flow

at Site: $20,000 \mathrm{ft}^{3} / \mathrm{s}$

Drinking Water:

Exposed Population: None

Fish:

Edible Harvest: (a)

Ditution Factor: $0.001^{(b)}$

(a) No fish catch data given in FES (1982), so generic consumption rates used (Table A-I).

(b) One percent of population obtain $10 \%$ of their fish from river according to FES (1972). 
POPULATION DOSE-COMMITMENT ESTIMATES ANO

AVERAGE INOIVIOUAL OOSE-COMMITMENT HISTOGRAM FOR

INDIAN POINT 1,2 AND 3

Dose Commitments (person-rem) from Waterborne Pathways

Total Body GI-LLI Thyroid Bone Liver

$\begin{array}{llllll}\text { Infant } & 0.0 \mathrm{E}+00 & 0.0 \mathrm{E}+00 & 0.0 \mathrm{E}+00 & 0.0 \mathrm{E}+00 & 0.0 \mathrm{E}+00 \\ \text { Child } & 2.7 \mathrm{E}-02 & 4.9 \mathrm{E}-03 & 2.3 \mathrm{E}-03 & 1.3 \mathrm{E}-0 \mathrm{1} & 1.4 \mathrm{E}-01 \\ \text { Teen } & 4.9 \mathrm{E}-02 & 1.0 \mathrm{E}-02 & 1.7 \mathrm{E}-03 & 7.7 \mathrm{E}-02 & 1.2 \mathrm{E}-01 \\ \text { Adu1t } & 5.2 \mathrm{E}-01 & 8.7 \mathrm{E}-02 & 1.1 \mathrm{E}-02 & 4.5 \mathrm{E}-01 & 7.2 \mathrm{E}-01 \\ \text { TOTAL } & 6.0 \mathrm{E}-01 & 1.0 \mathrm{E}-01 & 1.5 \mathrm{E}-02 & 6.5 \mathrm{E}-01 & 9.9 \mathrm{E}-01\end{array}$

Dose Commitments (person-rem) from Airborne Pathways

Total Body GI-LLI Thyroid Bone Liver Lung

$\begin{array}{lllllll}\text { Infant } & 3.7 \mathrm{E}-02 & 3.7 \mathrm{E}-02 & 6.0 \mathrm{E}-02 & 3.7 \mathrm{E}-02 & 3.8 \mathrm{E}-02 & 4.0 \mathrm{E}-02 \\ \text { Child } & 4.1 \mathrm{E}-01 & 4.1 \mathrm{E}-01 & 5.8 \mathrm{E}+01 & 4.2 \mathrm{E}-01 & 4.2 \mathrm{E}-01 & 4.6 \mathrm{E}-01 \\ \text { Teen } & 3.0 \mathrm{E}-01 & 3.0 \mathrm{E}-01 & 3.9 \mathrm{E}-01 & 3.0 \mathrm{E}-01 & 3.0 \mathrm{E}-01 & 3.6 \mathrm{E}-01 \\ \text { AduTt } & 1.8 \mathrm{E}+00 & 1.8 \mathrm{E}+00 & 2.2 \mathrm{E}+00 & 1.8 \mathrm{E}+00 & 1.8 \mathrm{E}+00 & 2.0 \mathrm{E}+00 \\ \text { TOTAL } & 2.6 \mathrm{E}+00 & 2.6 \mathrm{E}+00 & 3.2 \mathrm{E}+00 & 2.6 \mathrm{E}+00 & 2.6 \mathrm{E}+00 & 2.9 \mathrm{E}+00\end{array}$

Production/Consumption factors:

Produce: $<1 \quad$ Milk: $<1 \quad$ Meat: $<1$

Fraction of Population Receiving an Average Individual

Total-Body Dose Commitment from Airborne Pathways

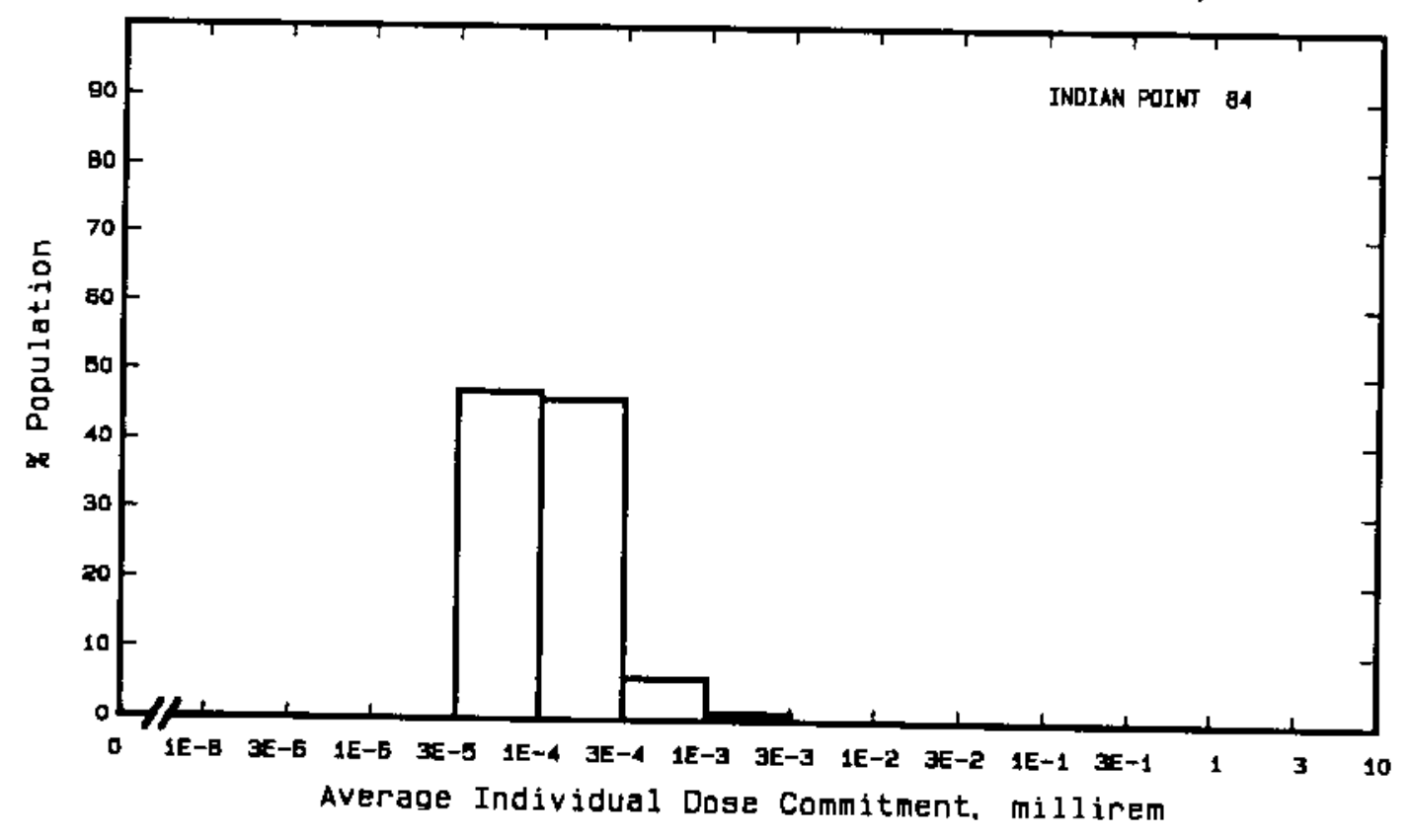


Site: KEWAUNEE

CARLTON, WISCONSIN

Location: $\quad$ N $44.3431^{\circ} \quad$ W $87.536^{\circ}$

POPULATION DATA

Total Population Within 2-to-80-km Region: 6.2E5

Major Metropolitan Centers Within Region:

\begin{tabular}{|c|c|c|c|}
\hline Center & Population & \multicolumn{2}{|c|}{ Location } \\
\hline Greenbay SMSA & 180,000 & $44 \mathrm{~km}$ & NW \\
\hline Appleton SMSA & 290,000 & $72 \mathrm{~km}$ & \\
\hline Sheboygan & 48,000 & $65 \mathrm{~km}$ & SSh \\
\hline Manitowoc & 33,000 & $29 \mathrm{~km}$ & SSh \\
\hline Neenah & 22,000 & $72 \mathrm{~km}$ & $W$ \\
\hline
\end{tabular}

SITE-SPECIFIC DATA - AIRBORNE PATHWAYS

Average Annual State Production

of Crops and Animal Products

In 80-km Radius Circle

Regional Productivity Factor:

Animal Grazing Factor:
Veg: $7.2 \mathrm{E} 7 \mathrm{kilogram}$

Milk: 1.2E9 liter

Meat: $1.0 \mathrm{E} 8$ kilogram

0.5

0.5

Meteorology Period of Record: 2 JAN 69 - 31 DEC 69 Recovery: 76\%

SITE-SPECIFIC DATA - WATERBORNE PATHWAYS via LAKE MICHIGAN

Average Dilution Flow from

Plant: $170 \mathrm{ft}^{3} / \mathrm{s}$

Drinking Water: $\quad$ Exposed Population: 260,090

Dilution Factor: $8.2 \mathrm{E}-3$

Fish:

Edible Harvest: $1.1^{(\mathrm{b})}(\mathrm{kg} / \mathrm{yr}$
Dilution Factor: $0.01^{(\mathrm{c})}$

(a) Drinking water dilution factor estimated by averaging dilution factors derived from FES (1972) suitably weighted for populations.

(b) Average individual consumption rate as given in FES used in lieu of catch data.

(c) Dilution factor reduced $1 / 10$ from that used in FES in consideration of lake mixing. 
POPULATION DOSE-COMMITMENT ESTIMATES AND

AVERAGE INOIVIOUAL OOSE-COMMITMENT HISTOGRAM FOR

\section{KEWAUNEE}

Oose Commitments (person-rem) from Waterborne Pathways

\begin{tabular}{|c|c|c|c|c|c|}
\hline & Total Body & GI-LLI & Thyroid & Bone & Liver \\
\hline $\begin{array}{l}\text { Infant } \\
\text { Child } \\
\text { Teen } \\
\text { Adult }\end{array}$ & $\begin{array}{l}5.2 \mathrm{E}-03 \\
7.0 \mathrm{E}-02 \\
4.5 \mathrm{E}-02 \\
4.3 \mathrm{E}-01\end{array}$ & $\begin{array}{l}5.4 \mathrm{E}-03 \\
6.9 \mathrm{E}-02 \\
3.8 \mathrm{E}-02 \\
3.3 \mathrm{E}-01\end{array}$ & $\begin{array}{l}4.8 \mathrm{E}-03 \\
5.4 \mathrm{E}-02 \\
2.1 \mathrm{E}-02 \\
1.8 \mathrm{E}-01\end{array}$ & $\begin{array}{l}9.9 \mathrm{E}-04 \\
6.5 \mathrm{E}-02 \\
3.5 \mathrm{E}-02 \\
2.1 \mathrm{E}-01\end{array}$ & $\begin{array}{l}5.7 \mathrm{E}-03 \\
1.2 \mathrm{E}-01 \\
7.7 \mathrm{E}-02 \\
5.1 \mathrm{E}-01\end{array}$ \\
\hline TOTAL & $5.5 E-01$ & $4.5 E-01$ & $2.6 \mathrm{E}-01$ & $3.1 E-0 I$ & $7.2 \mathrm{E}-01$ \\
\hline
\end{tabular}

Dose Commitments (person-rem) from Airborne Pathways

Total Body GI-LLI Thyroid Bone Liver Lung

$\begin{array}{lllllll}\text { Infant } & 1.3 \mathrm{E}-05 & 1.0 \mathrm{E}-05 & 4.7 \mathrm{E}-04 & 2.6 \mathrm{E}-05 & 1.1 \mathrm{E}-05 & 1.4 \mathrm{E}-05 \\ \text { Chi1d } & 2.6 \mathrm{E}-04 & 1.4 \mathrm{E}-04 & 3.5 \mathrm{E}-03 & 8.3 \mathrm{E}-04 & 1.4 \mathrm{E}-04 & 1.9 \mathrm{E}-04 \\ \text { Teen } & 1.5 \mathrm{E}-04 & 9.6 \mathrm{E}-05 & 1.6 \mathrm{E}-03 & 4.8 \mathrm{E}-04 & 9.1 \mathrm{E}-05 & 1.4 \mathrm{E}-04 \\ \text { Adu1t } & 7.8 \mathrm{E}-04 & 5.5 \mathrm{E}-04 & 6.5 \mathrm{E}-03 & 2.5 \mathrm{E}-03 & 5.2 \mathrm{E}-04 & 6.9 \mathrm{E}-04 \\ \text { TOTAL } & 1.2 \mathrm{E}-03 & 7.9 \mathrm{E}-04 & 1.2 \mathrm{E}-02 & 3.8 \mathrm{E}-03 & 7.6 \mathrm{E}-04 & 1.0 \mathrm{E}-03\end{array}$

Production/Consumption factors:

Produce: <1 Milk: 7.2 Meat: 1.0

Fraction of Population Receiving an Average Individual

Total-Body Dose Commitment from Airborne Pathways

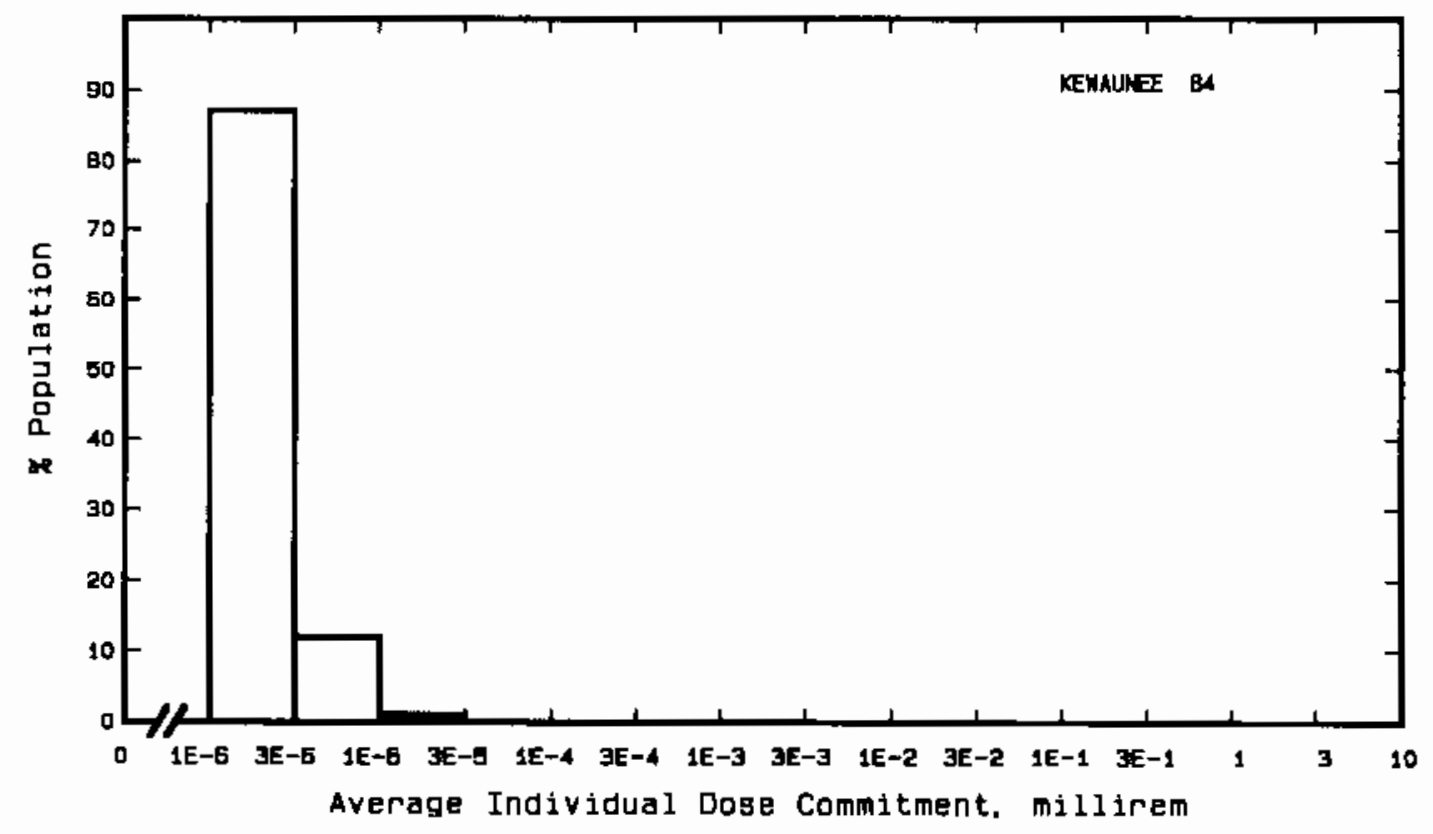


Site: LACROSSE

GENOA, WISCONSIN

Location: $\quad N 43.5583^{\circ} \quad$ W $91.2306^{\circ}$

POPULATION DATA

Total Population Within 2-to-80-km Region: 3.5E5

Major Metropolitan Centers Within Region:

Center Population Location

La Crosse SMSA $\quad 92,000 \quad 27 \mathrm{~km} \quad \mathrm{~N}$

Winona $\quad 25,000 \quad 64 \mathrm{~km}$ WNW

SITE-SPECIFIC DATA - AIRBORNE PATHWAYS

Average Annual State Production

of Crops and Animal Products

In $80-\mathrm{km}$ Radius Circle

Regional Productivity Factor:

Animal Grazing Factor:

Meteorology Period of Record: I JAN 75 - 31 DEC 75 Recovery: 97\%

SITE-SPECIFIC DATA - WATERBORNE PATHWAYS via MISSISSIPPI RIVER

Average River

Flow at Site: $28,000 \mathrm{ft}^{3} / \mathrm{s}$

Drinking Water:

Exposed Poptiation: None

Fish:

Edible Harvest: (a)

DiTution Factor: $0.5^{(a)}$

(a) No fish catch data given in FES (1976), so 1/2 population assumed to consume river fish at generic consumption rates (Table A-1). 
POPULATION DOSE-COMMITMENT ESTIMATES AND

AVERAGE INDIVIDUAL DOSE-COMMITMENT HISTOGRAM FOR

\section{LACROSSE}

Dose Commitments (person-rem) from Waterborne Pathways

Total Body GI-LLI Thyroid Bone Liver

$\begin{array}{llllll}\text { Infant } & 0.0 \mathrm{E}+00 & 0.0 \mathrm{E}+00 & 0.0 \mathrm{E}+00 & 0.0 \mathrm{E}+00 & 0.0 \mathrm{E}+00 \\ \text { Child } & 2.5 \mathrm{E}-02 & 3.2 \mathrm{E}-02 & 2.7 \mathrm{E}-03 & 1.0 \mathrm{E}-01 & 1.1 \mathrm{E}-01 \\ \text { Teen } & 3.6 \mathrm{E}-02 & 6.8 \mathrm{E}-02 & 1.9 \mathrm{E}-03 & 6.2 \mathrm{E}-02 & 9.3 \mathrm{E}-02 \\ \text { Adult } & 3.6 \mathrm{E}-01 & 6.0 \mathrm{E}-01 & 1.3 \mathrm{E}-02 & 3.6 \mathrm{E}-01 & 5.5 \mathrm{E}-02 \\ \text { TOTAL } & 4.2 \mathrm{E}-01 & 7.0 \mathrm{E}-01 & 1.7 \mathrm{E}-02 & 5.2 \mathrm{E}-01 & 7.5 \mathrm{E}-01\end{array}$

Dose Commitments (person-rem) from Airborne Pathways

Total Body GI-LLI Thyroid Bone Liver Lung

$\begin{array}{lllllll}\text { Infant } & 9.1 \mathrm{E}-03 & 9.1 \mathrm{E}-03 & 9.8 \mathrm{E}-03 & 9.1 \mathrm{E}-03 & 9.1 \mathrm{E}-03 & 9.4 \mathrm{E}-03 \\ \text { Child } & 1.0 \mathrm{E}-01 & 1.0 \mathrm{E}-01 & 1.1 \mathrm{E}-01 & 1.0 \mathrm{E}-01 & 1.0 \mathrm{E}-01 & 1.1 \mathrm{E}-01 \\ \text { Teen } & 7.5 \mathrm{E}-02 & 7.5 \mathrm{E}-02 & 7.8 \mathrm{E}-02 & 7.4 \mathrm{E}-02 & 7.5 \mathrm{E}-02 & 7.9 \mathrm{E}-02 \\ \text { Adult } & 4.5 \mathrm{E}-01 & 4.5 \mathrm{E}-01 & 4.6 \mathrm{E}-01 & 4.5 \mathrm{E}-01 & 4.5 \mathrm{E}-01 & 4.7 \mathrm{E}-01 \\ \text { TOTAL } & 6.4 \mathrm{E}-01 & 6.4 \mathrm{E}-01 & 6.6 \mathrm{E}-01 & 6.3 \mathrm{E}-01 & 6.4 \mathrm{E}-01 & 6.6 \mathrm{E}-01\end{array}$

Production/Consumption factors:

Produce: $1.1 \quad$ Milk: $26 \quad$ Meat: 3.7

Fraction of Population Receiving an Average Individual

Total-Body Dose Commitment from Airborne Pathways

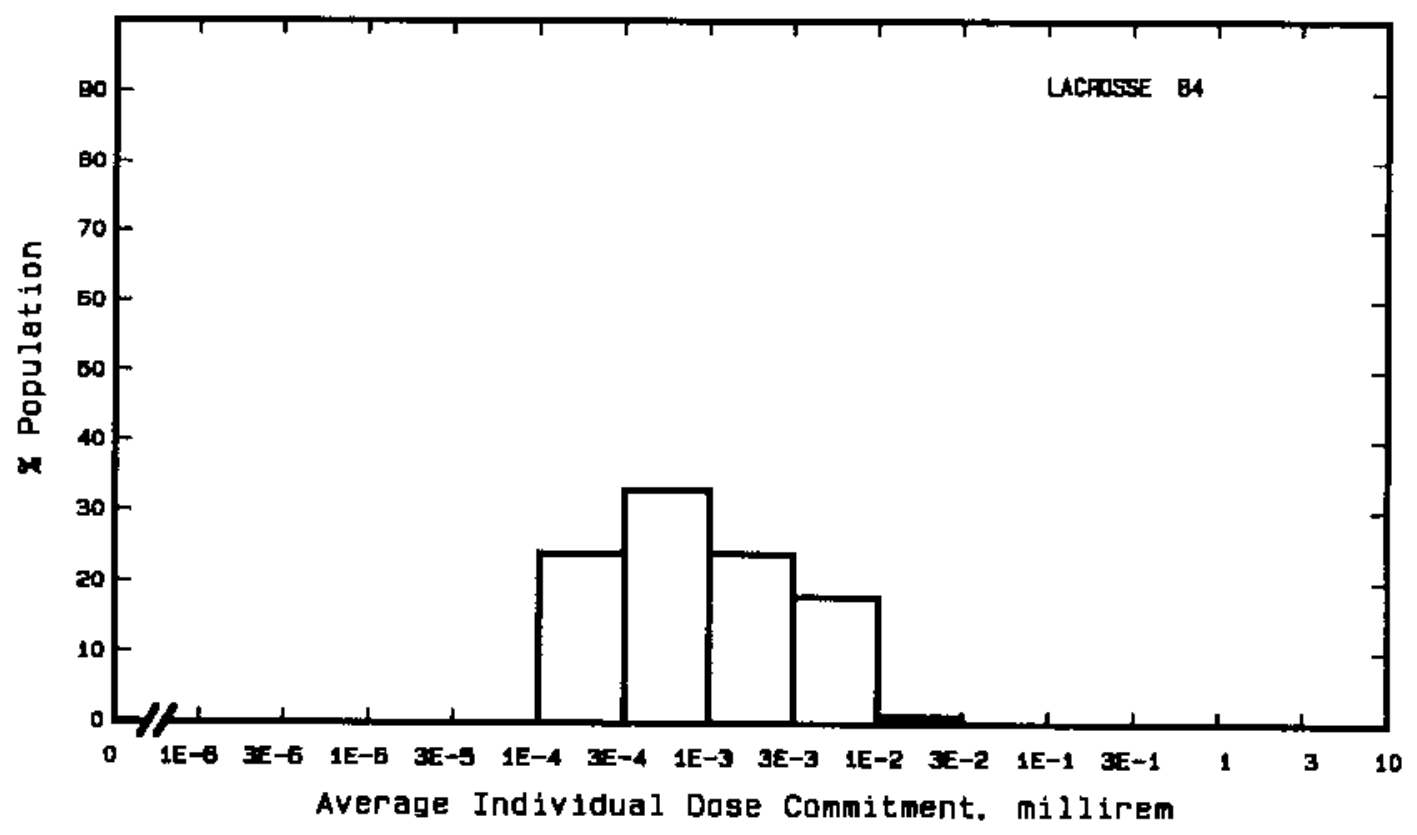


Site: LASALLE

SENECA, ILLINOIS

Location: N $41.2439^{\circ} \quad$ W $88.6708^{\circ}$

POPULATION DATA

Total Population Within 2-to-80-km Region: 1.0E6

Major Metropotitan Centers Within Region:

\begin{tabular}{lcccc} 
Center & Population & \multicolumn{2}{c}{ Location } \\
\cline { 2 - 5 } Joliet & 78,000 & & $59 \mathrm{~km}$ & NNE \\
Aurora & 80,000 & $65 \mathrm{~km}$ & NNE \\
Kankakee & 103,000 & $69 \mathrm{~km}$ & ESE \\
Dekalb & 33,000 & $77 \mathrm{~km}$ & N \\
Napervitle & 42,000 & $73 \mathrm{~km}$ & NE
\end{tabular}

SITE-SPECIFIC DATA - AIR8ORNE PATHWAYS

Average Annua State Production

of Crops and Animal Products

In 80-km Radius Circle

Veg: $1.1 E 8$ kiTogram

MiTk: $1.8 \mathrm{E} 8$ Titer

Meat: $1.9 E 8$ kilogram

Regional Productivity Factor:

Animal Grazing Factor:

0.9

0.5

Meteorology Period of Record: 1 Jan $82-31$ Dec 82 Recovery: $99 \%$

SITE-SPECIFIC DATA - WATERBORNE PATHWAYS via ILLINOIS RIVER

Average River Flow

at site: $1_{. .}^{-1}, 000 \mathrm{ft}^{3} / \mathrm{s}$

Drinking Water:

Exposed Poputation: None

Dilution Factor:

Fish:

Edible Harvest: None ${ }^{(a)}$

DiTution Factor:

(a) Because of river quality, no fish consumption is considered according to FES (1973). 


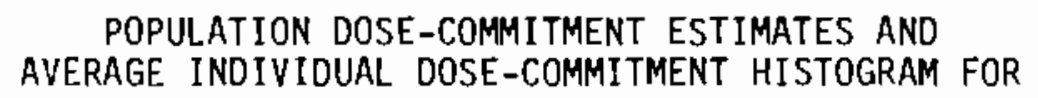

LASALLE 1 and 2

Dose Commitments (person-rem) from Waterborne Pathways

Total Body GI-LII Thyroid Bone $\underline{\underline{\text { Liver }}}$

Infant

Child

Teen

Adult

(Little or No Waterborne Pathway Doses)

TOTAL

Dose Commitments (person-rem) from Airborne Pathways

Total Body GI-LII Thyroid Bone Liver Lung

Infant

3. $9 \mathrm{E}-04$

3. $9 \mathrm{E}-04$

2.6E-03

4. $5 \mathrm{E}-03$

4.6E-03

2. $2 \mathrm{E}-02$

3.7E-D4

3. $9 \mathrm{E}-04$

4. $1 \mathrm{E}-04$

Teen

3. $2 \mathrm{E}-03$

$3.4 \mathrm{E}-03$

1. $0 \mathrm{E}-02$

4. IE-03

4. $5 \mathrm{E}-03$

3. $0 \mathrm{E}-03$

3. $2 \mathrm{E}-03$

4. $9 E-03$

Adult

2.0E-02

4. 4E-02

1. $8 \mathrm{E}-02$

1.9E-02

3.7E-03

TOTAL

2.7E-02

2.9E-02 7.9E-02

2.5E-02

2.7E-02

2.1E-02

3. $0 \mathrm{E}-02$

Production/Consumption factors:
Produce:
0.5
MiTk: 1.2
Meat: 2.0
Fraction of Population Receiving an Average Individual Total-Body Dose Commitment from Airborne Pathways

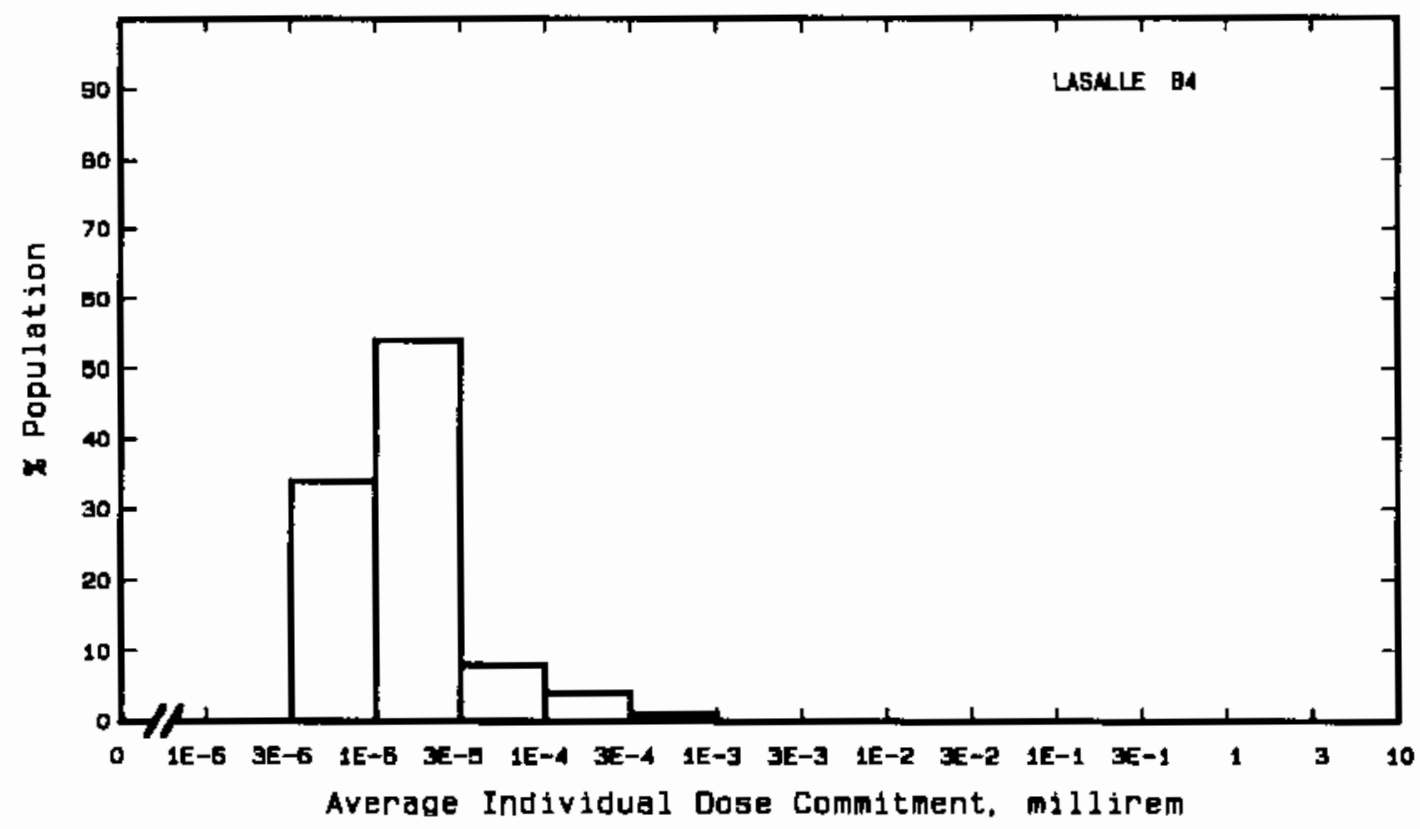


Site: LIMERICK

POTTSTOWN, PENNSYLVANIA

Location: N $\quad$ N0.2242 $\quad$ W $75.5875^{\circ}$

POPULATION DATA

Total Population Within 2-to-80-km Region: $6.8 \mathrm{E} 6$

Major Metropolitan Centers Within Region:

Center

Philadelphia PMSA

Allentown-Bethlehem MSA

Reading MSA

Lancaster MSA

Wilmington PMSA

Camion

Trenton PMSA
Population!

$4,800,000$

65,000

310,000

360,000

540,000

110,000

310,000

\section{Location}

$40 \mathrm{~km} \quad$ ESE

$42 \mathrm{~km}$ ENE

$34 \mathrm{~km}$ WNW

$68 \mathrm{~km}$ WSW

$56 \mathrm{~km} \mathrm{~S}$

$52 \mathrm{~km} \quad \mathrm{SE}$

$71 \mathrm{~km} \quad \mathrm{E}$

SITE-SPECIFIC DATA - AIRBORNE PATHWAYS

Average Annual State Production

of Crops and Animal Products

In 80-km Radius Circle

Regional Productivity Factor:

Animal Grazing Factor:

Meteorology Period of Record: 1 Jan $74-31$ Dec 74 Recovery: 93\%
Veg: $5.3 \mathrm{E} 7$ kiTogram

MiTk: 5.3E8 Titer

Meat: $5.4 \mathrm{E} 7$ ki logram

0.9

0.6

SITE-SPECIFIC DATA - WATERBORNE PATHWAYS via SCHUYLKILL

Average River Flow at site: $3,000 \mathrm{ft}^{3} \mathrm{~s}$

Drinking Water:

Exposed Population: $3 E 6$

Dilution Factor: 1

Fish:

Edible Harvest: None 


\section{POPULATION DOSE-COMMITMENT ESTIMATES AND AVERAGE INDIVIDUAL DOSE-COMMITMENT HISTOGRAM FOR \\ LIMERICK}

Dose Commitments (person-rem) from Waterborne Pathways

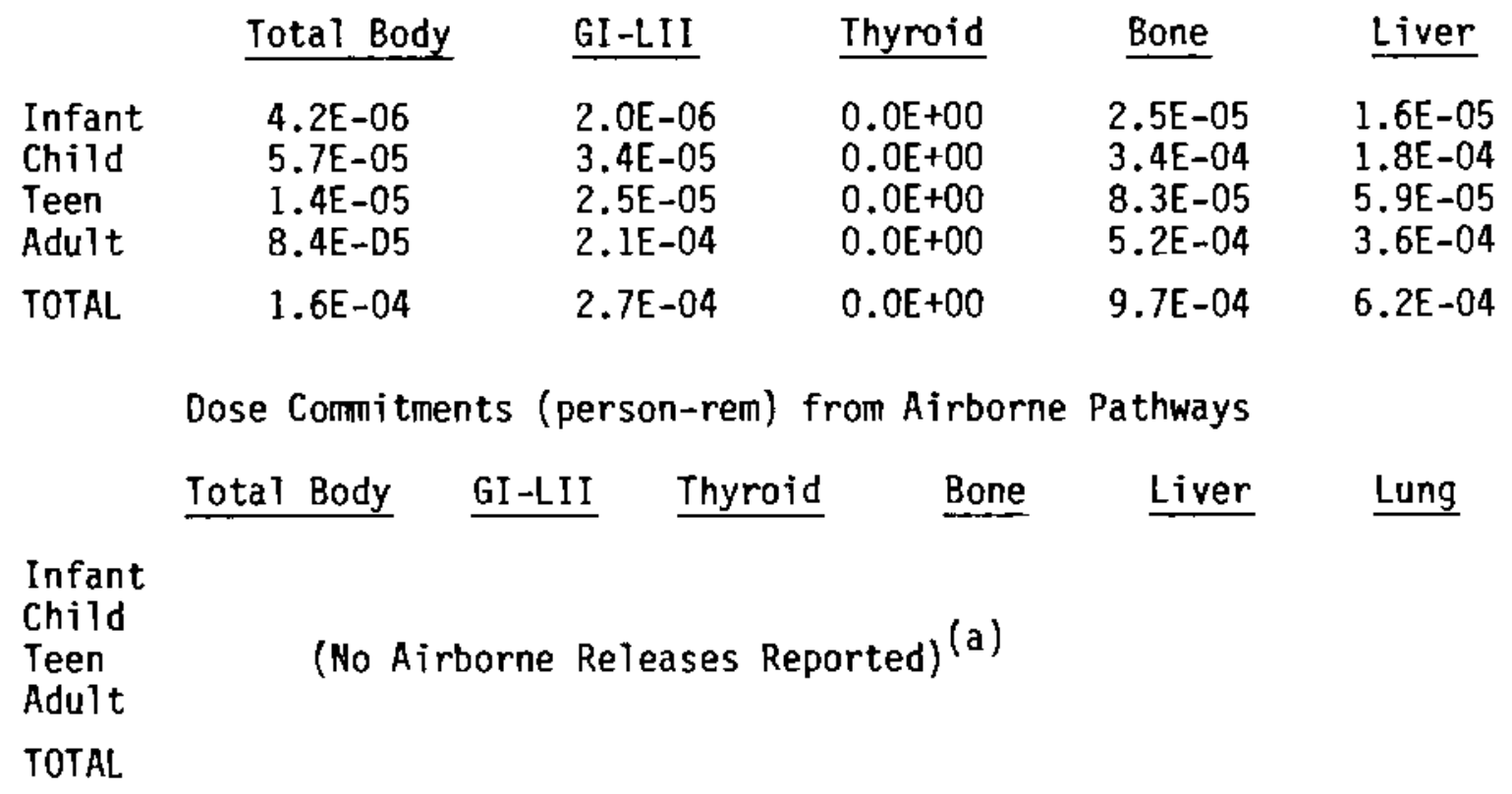

Production/Consumption factors:

Produce: Milk: Meat:

(a) Tichler, et al. 1987. 
Site: MAINE YANKEE

LINCOLN COUNTY, MAINE

Location: $\quad$ N $43.9506^{\circ} \quad$ W $69.6961^{\circ}$

POPULATION DATA

Total Population Within 2-to-80-km Region: 6.0E5

Major Metropolitan Centers Within Region:

Center Population Location

Portland SMSA $\quad 180,000 \quad 56 \mathrm{~km}$ WSW

Lewiston-Auburn SMSA $72,000 \quad 45 \mathrm{~km} \quad$ WNW

Augusta $\quad 22,000 \quad 41 \mathrm{~km} \quad \mathrm{~N}$

Biddeford $\quad 20,000 \quad 80 \mathrm{~km} \quad \mathrm{SW}$

Waterville $1 \mathrm{~B}, 000 \quad 67 \mathrm{~km} \quad \mathrm{~N}$

SITE-SPECIFIC DATA - AIRBORNE PATHWAYS

Average Annual State Production

of Crops and Animal Products

In 80-km Radius Circle

Regional Productivity Factor:

Animal Grazing Factor:

Meteorology Period of Record: 1 APR 75 - 31 MAR 76 Recovery: 98\%
Veg: $2.4 E 8$ kilogram

Milk: $6.6 \mathrm{E} 7$ liter

Meat: $4.3 E 6$ kilogram

0.6

0.5

SITE-SPECIFIC DATA - WATERBORNE PATHWAYS via ATLANTIC OCEAN

Average Dilution Flow

from Plant: $740 \mathrm{ft}^{3} / \mathrm{s}$

Fish:

Edible Harvest: (a)

Dilution Factor: 0.001

Invertebrates:

Edible Harvest: (a)

Oilution Factor: 0.002

(a) No seafood harvest data given in FES (1972), thus generic population consumption rates used (Table A-I). 
POPULATION DOSE-COMMITMENT ESTIMATES AND

AVERAGE INDIVIDUAL DOSE-COMMITMENT HISTOGRAM FOR

\section{MAINE YANKEE}

Dose Cormitments (person-rem) from Waterborne Pathways

Total Body GI-LLI Thyroid Bone Liver

$\begin{array}{llllll}\text { Infant } & 0.0 \mathrm{E}+00 & 0.0 \mathrm{E}+00 & 0.0 \mathrm{E}+00 & 0.0 \mathrm{E}+00 & 0.0 \mathrm{E}+00 \\ \text { Child } & 7 . \mathrm{BE}-05 & 1.8 \mathrm{E}-04 & 6.0 \mathrm{E}-05 & 5.6 \mathrm{E}-05 & 7.6 \mathrm{E}-05 \\ \text { Teen } & 6.5 \mathrm{E}-05 & 3.6 \mathrm{E}-04 & 4.5 \mathrm{E}-05 & 3.5 \mathrm{E}-05 & 6.5 \mathrm{E}-05 \\ \text { Adult } & 4.8 \mathrm{E}-04 & 3.2 \mathrm{E}-03 & 3.1 \mathrm{E}-04 & 2.1 \mathrm{E}-04 & 4.1 \mathrm{E}-04 \\ \text { TOTAL } & 6.2 \mathrm{E}-04 & 3.7 \mathrm{E}-03 & 4.2 \mathrm{E}-04 & 3.0 \mathrm{E}-04 & 5.5 \mathrm{E}-04\end{array}$

Dose Commitments (person-rem) from Airborne Pathways

Total Body GI-LLI Thyroid Bone Liver Lung

$\begin{array}{lllllll}\text { Infant } & 6.3 \mathrm{E}-05 & 6.4 \mathrm{E}-05 & 4.7 \mathrm{E}-04 & 4.8 \mathrm{E}-05 & 6.5 \mathrm{E}-05 & 7.3 \mathrm{E}-05 \\ \text { Child } & 1.0 \mathrm{E}-03 & 1.1 \mathrm{E}-03 & 7.1 \mathrm{E}-03 & 5.6 \mathrm{E}-04 & 1.1 \mathrm{E}-03 & 1.2 \mathrm{E}-03 \\ \text { Teen } & 6.7 \mathrm{E}-04 & 7.3 \mathrm{E}-04 & 3.2 \mathrm{E}-03 & 3.9 \mathrm{E}-04 & 6.9 \mathrm{E}-04 & 8.3 \mathrm{E}-04 \\ \text { Adult } & 3.8 \mathrm{E}-03 & 4.1 \mathrm{E}-03 & 1.4 \mathrm{E}-02 & 2.3 \mathrm{E}-03 & 3.8 \mathrm{E}-03 & 4.4 \mathrm{E}-03 \\ \text { ToTAL } & 5.6 \mathrm{E}-03 & 5.9 \mathrm{E}-03 & 2.5 \mathrm{E}-02 & 3.3 \mathrm{E}-03 & 5.7 \mathrm{E}-03 & 6.5 \mathrm{E}-03\end{array}$

Production/Consumption factors:

Produce: 1.2 Milk: <1 Meat: <1

Fraction of Population Receiving an Average Individual

Total-Body Dase Commitment from Atrborne Pathways

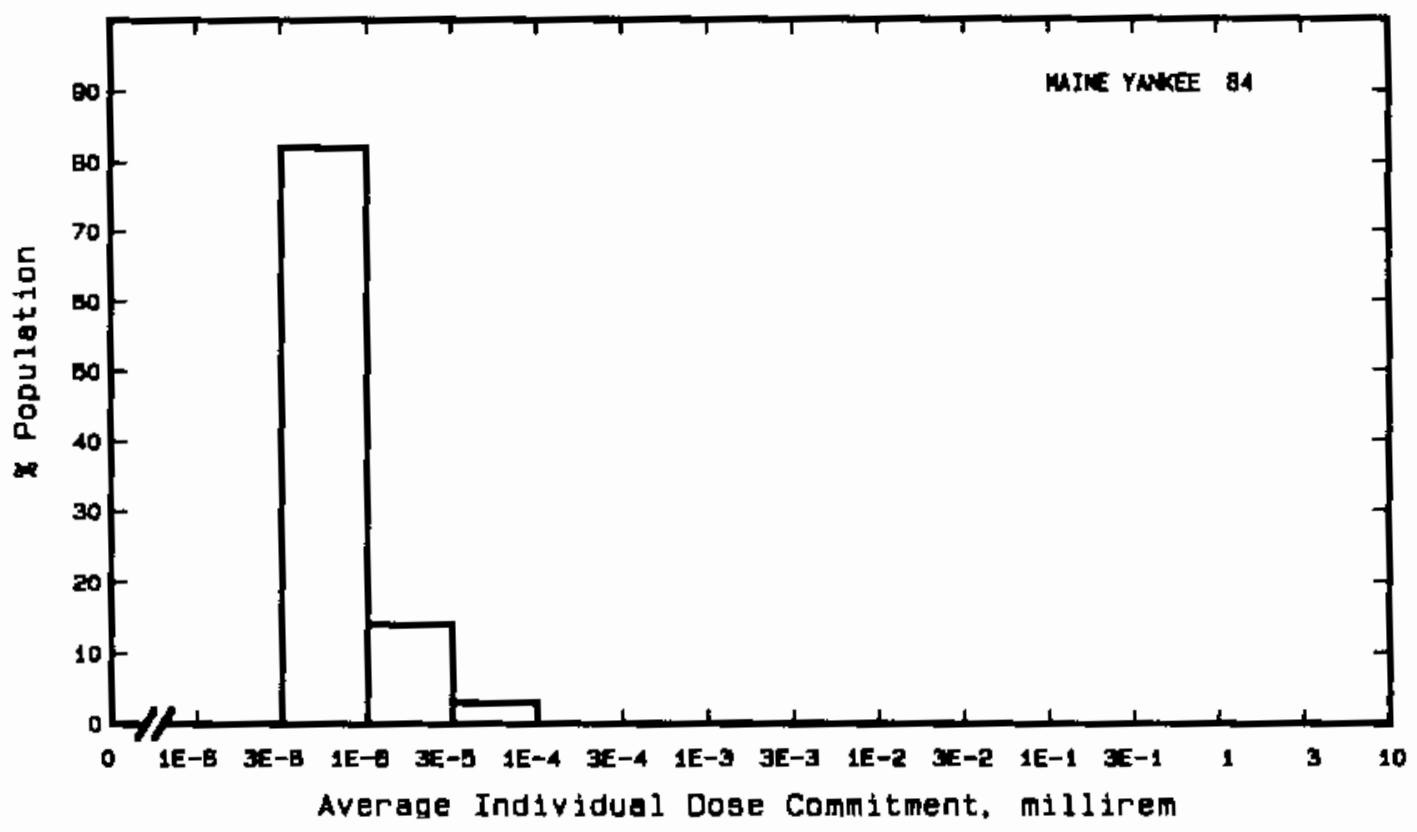


Site: MCGUIRE

Location: N $35.4322^{\circ}$ W $80.9483^{\circ}$

POPULATION OATA

Total Population Within 2-to-80-km Region: 1.7 E6

Major Metropolitan Centers Within Region:

Center Population Location

Charlotte-Gastonia SMSA $\quad 660,000 \quad 25 \mathrm{~km} \quad \mathrm{~S}$

Rockhill $\quad 36,000 \quad 57 \mathrm{~km} \quad \mathrm{~S}$

Kannapolis $\quad 36,000 \quad 30 \mathrm{~km} \quad E$

Salisbury $\quad 24,000 \quad 51 \mathrm{~km}$ ENE

Hickory $22,000 \quad 49 \mathrm{~km}$ NW

SITE-SPECIFIC DATA - AIRBORNE PATHWAYS

Average Annual State Production

of Crops and Animal Products

In 80-km Radius Circle

Regional Productivity Factor:

Animal Grazing Factor:

Meteorology Period of Record: 17 OCT $70-16$ OCT 71 Recovery: 90\%

SITE-SPECIFIC OATA - WATERBORNE PATHWAYS via LAKE NORMAN ON CATAWBA RIVER

Average River

Flow at Site: $2,600 \mathrm{ft}^{3} / \mathrm{s}$

Drinking Water:

Exposed Population: 720,000

Fish:

Edible Harvest: $1.8^{(\mathrm{a})} \mathrm{kg} / \mathrm{yr}$

DiTution Factor: 1

(a) Average individual consumption rate as given in FES (1976). 
POPULATION DOSE-COMMITMENT ESTIMATES AND

AVERAGE INDIVIDUAL OOSE-COMMITMENT HISTOGRAM FOR

MCGUIRE 1 AND 2

Dose Commitments (person-rem) from Waterborne Pathways

Total Body GI-LLI Thyroid Bone Liver

$\begin{array}{llllll}\text { Infant } & 1.7 \mathrm{E}-0 \mathrm{I} & 1.7 \mathrm{E}-01 & 1.2 \mathrm{E}+00 & 3.6 \mathrm{E}-02 & 2.0 \mathrm{E}-01 \\ \text { Child } & 2.4 \mathrm{E}+00 & 2.7 \mathrm{E}+00 & 9.2 \mathrm{E}+00 & 2.6 \mathrm{E}+00 & 4.6 \mathrm{E}+00 \\ \text { Teen } & 1.6 \mathrm{E}+00 & 2.4 \mathrm{E}+00 & 3.1 \mathrm{E}+00 & 1.4 \mathrm{E}+00 & 2.9 \mathrm{E}+00 \\ \text { Adult } & 1.5 \mathrm{E}+01 & 2.1 \mathrm{E}+01 & 2.3 \mathrm{E}+01 & 8.1 \mathrm{E}+00 & 1.9 \mathrm{E}+01 \\ \text { T0TAL } & 2.0 \mathrm{E}+01 & 2.6 \mathrm{E}+01 & 3.6 \mathrm{E}+01 & 1.2 \mathrm{E}+01 & 2.7 \mathrm{E}+01\end{array}$

Dose Commitments (person-rem) from Airborne Pathways

Total Body GI-LLI Thyroid Bone Liver Lung

$\begin{array}{lllllll}\text { Infant } & 2.5 \mathrm{E}-03 & 2.5 \mathrm{E}-03 & 1.5 \mathrm{E}-02 & 2.4 \mathrm{E}-03 & 2.5 \mathrm{E}-03 & 2.6 \mathrm{E}-03 \\ \text { Child } & 2.8 \mathrm{E}-02 & 2.8 \mathrm{E}-02 & 1.0 \mathrm{E}-01 & 2.6 \mathrm{E}-02 & 2 . \mathrm{BE}-02 & 3.1 \mathrm{E}-02 \\ \text { Teen } & 2.1 \mathrm{E}-02 & 2.0 \mathrm{E}-02 & 5.2 \mathrm{E}-02 & 1.9 \mathrm{E}-02 & 2.1 \mathrm{E}-02 & 2.4 \mathrm{E}-02 \\ \text { Adult } & 1.2 \mathrm{E}-01 & 1.2 \mathrm{E}-01 & 2.3 \mathrm{E}-01 & 1.1 \mathrm{E}-01 & 1.2 \mathrm{E}-01 & 1.3 \mathrm{E}-01 \\ \text { TOTAL } & 1.7 \mathrm{E}-01 & 1.7 \mathrm{E}-01 & 4.1 \mathrm{E}-01 & 1.6 \mathrm{E}-01 & 1.8 \mathrm{E}-01 & 1.9 \mathrm{E}-01\end{array}$

Production/Consumption factors:

Produce: $<1$ Milk: $<1 \quad$ Meat: $<1$

Fraction of Population Receiving an Average Individurl

Total-Body Dose Commitment from Airborne Pathways

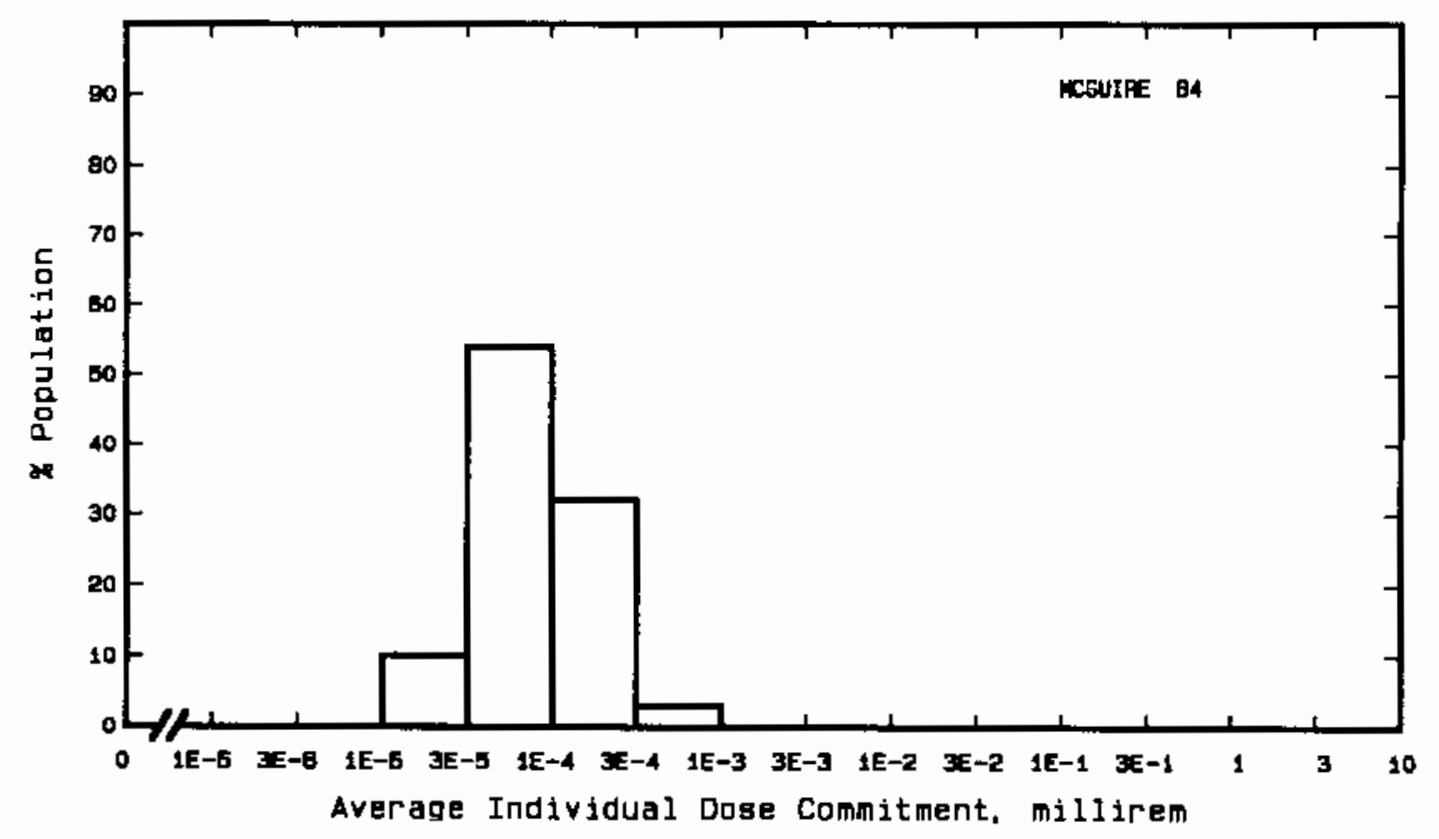


Site: MILLSTONE

WATERFJRD, CONNECTICUT

Location: $\quad \mathrm{N} 41.3086^{\circ} \quad$ W $72.1631^{\circ}$

POPULATION DATA

Total PopuTation Within 2-to-80-km Region: 2.5E6

Major Metropolitan Centers Within Region:

Center

Hartford SMSA

New Haven-West Haven SMSA

New London-Norwich SMSA

New Britain SMSA

Wa terbury SMSA
Population

730,000

420,000

250,000

140,000

230,000

\section{Location}

$67 \mathrm{~km} \quad \mathrm{NW}$

$64 \mathrm{~km} \quad \mathrm{~W}$

$8 \mathrm{~km} \quad \mathrm{NNE}$

$65 \mathrm{~km} \quad \mathrm{NW}$

$78 \mathrm{~km} \quad W N W$

SITE-SPECIFIC DATA - AIRBORNE PATHWAYS

Average Annual State Production

of Crops and Animal Products

In $80-\mathrm{km}$ Radius Circle

Regional Productivity Factor:

Animal Grazing Factor:

Meteorology Period of Record: 1 JAN 74 - 31 DEC 74 Recovery: 95\%
Veg: $3.2 E 7$ kilogram

MiTk: 4.4E8 Titer

Meat: $2.0 \mathrm{E} 7$ kilogram

0.6

0.6

SITE-SPECIFIC DATA - WATERBORNE PATHWAYS via NIANTIC BAY

Average Dilution Flow

from Plant: $4,100 \mathrm{ft}^{3} / \mathrm{s}$

Fish:

Edible Harvest: $9.1 \mathrm{E} 4 \mathrm{~kg} / \mathrm{yr}$

Dilution Factor: 0.001

Invertebrates:

Edible Harvest: $9.1 \mathrm{E} 4 \mathrm{~kg} / \mathrm{yr}$

Dilution Factor: 0.002 
POPULATION DOSE-COMMITMENT ESTIMATES AND

AVERAGE INDIVIDUAL DOSE-COMMITMENT HISTOGRAM FOR

MILLSTONE 1 ANO 2

Dose Commitments (person-rem) from Waterborne Pathways

\begin{tabular}{|c|c|c|c|c|c|}
\hline & Total Body & GI-LLI & Thyroid & Bone & Liver \\
\hline $\begin{array}{l}\text { Infant } \\
\text { Child } \\
\text { Teen } \\
\text { AduTt }\end{array}$ & $\begin{array}{l}0.0 \mathrm{E}+00 \\
1.6 \mathrm{E}-03 \\
2.5 \mathrm{E}-03 \\
2.6 \mathrm{E}-02\end{array}$ & $\begin{array}{l}0.0 \mathrm{E}+00 \\
1.5 \mathrm{E}-03 \\
3.2 \mathrm{E}-03 \\
2.8 \mathrm{E}-02\end{array}$ & $\begin{array}{l}0.0 \mathrm{E}+00 \\
2.0 \mathrm{E}-03 \\
1.5 \mathrm{E}-03 \\
9.7 \mathrm{E}-03\end{array}$ & $\begin{array}{l}0.0 E+00 \\
5.9 E-03 \\
3.5 E-03 \\
2.0 E-02\end{array}$ & $\begin{array}{l}0.0 \mathrm{E}+00 \\
6.8 \mathrm{E}-03 \\
5.8 \mathrm{E}-03 \\
3.4 \mathrm{E}-02\end{array}$ \\
\hline TOTAL & $3.0 \mathrm{E}-02$ & 3. $3 \mathrm{E}-02$ & 1. $3 \mathrm{E}-02$ & $3.0 \mathrm{E}-02$ & $4.7 \mathrm{E}-02$ \\
\hline
\end{tabular}

Dose Commitments (person-rem) from Airborne Pathways

Total Body GI-LLI Thyroid Bone Liver Lung

$\begin{array}{lllllll}\text { Infant } & 1.9 \mathrm{E}-02 & 1.9 \mathrm{E}-02 & 2.0 \mathrm{E}-01 & 1.8 \mathrm{E}-02 & 2.2 \mathrm{E}-02 & 2.0 \mathrm{E}-02 \\ \text { Child } & 2.1 \mathrm{E}-01 & 2.1 \mathrm{E}-01 & 1.1 \mathrm{E}+00 & 1.9 \mathrm{E}-01 & 2.2 \mathrm{E}-01 & 2.3 \mathrm{E}-01 \\ \text { Teen } & 1.5 \mathrm{E}-01 & 1.5 \mathrm{E}-01 & 5.2 \mathrm{E}-01 & 1.4 \mathrm{E}-01 & 1.6 \mathrm{E}-01 & 1.8 \mathrm{E}-01 \\ \text { Adult } & 9.2 \mathrm{E}-01 & 9.2 \mathrm{E}-01 & 2.1 \mathrm{E}+00 & 8.4 \mathrm{E}-01 & 9.2 \mathrm{E}-01 & 9.9 \mathrm{E}-01 \\ \text { TOTAL } & 1.3 \mathrm{E}-00 & 1.3 \mathrm{E}-00 & 3.9 \mathrm{E}+00 & 1.2 \mathrm{E}+00 & 1.3 \mathrm{E}+00 & 1.4 \mathrm{E}+00\end{array}$

Production/Consumption factors:

Produce: <1 Milk: <1 Meat: <1

Fraction of Population Receiving an Average Individual Total-Body Cose Commitment from Airborne Pathways

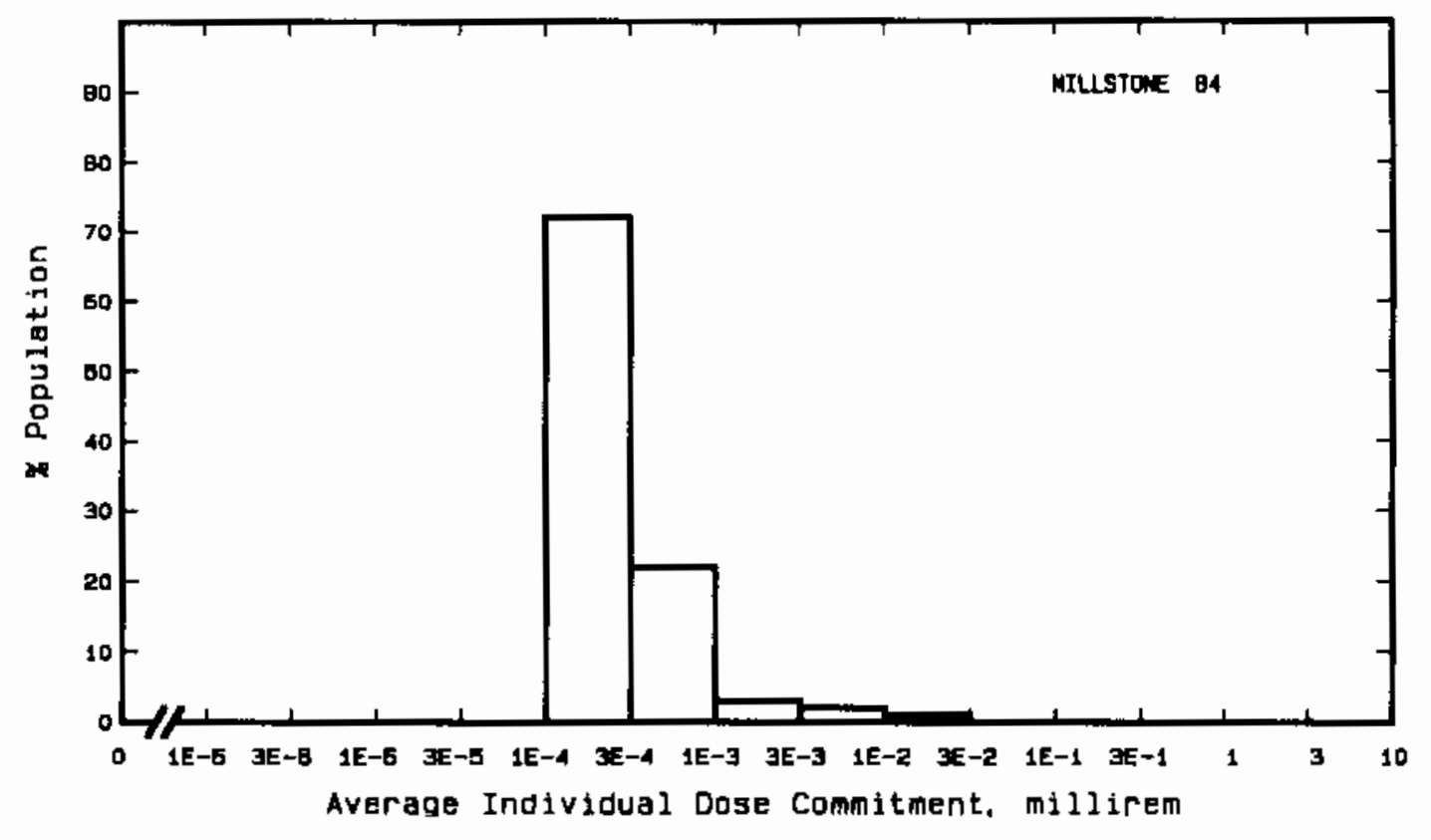


Site: MONTICELLO

MONTICELLO, MINNESOTA

Location: N $45.3333^{\circ}$ W $93.8433^{\circ}$

POPULATION DATA

Total Population Within 2-to-80-km Region: 2.2E6

Major Metropolitan Centers Within Region:

Center Population Location

Minneapotis-St. Paut SMSA $2,100,000 \quad 60 \mathrm{~km} \quad$ SE

St. Cloud SMSA $\quad 160,000 \quad 36 \mathrm{~km}$ NW

Bloomington $\quad 83,000 \quad 72 \mathrm{~km}$ SE

Edina $\quad 47,000 \quad 63 \mathrm{~km} \quad \mathrm{SE}$

Richfield $\quad 39,000 \quad 67 \mathrm{~km} \quad \mathrm{SE}$

SITE-SPECIFIC DATA - AIRBORNE PATHWAYS

Average Annual State Production

Of Crops and Animal Products

In $80-\mathrm{km}$ Radius Circle

Veg: 1.2E8 kiTogram

Milk: 4.0E8 Titer

Regional Productivity Factor:

Animat Grazing Factor:

Meat: l.1E8 ki Togram

Meteorology Period of Record: 1 JAN 74 - 31 DEC 74 Recovery: 92\%

SITE-SPECIFIC DATA - WATERBORNE PATHWAYS via MISSISSIPPI RIVER

Average River Flow

at Site: $4,600 \mathrm{ft}^{3} / \mathrm{s}$

Drinking Water: Exposed Population: (a)

Fish: Edible Harvest: (a)

(a) No radionuclides released in liquid effluents reported (Tichler, et a1. 1987). 
POPULATION DOSE-COMMITMENT ESTIMATES ANO

AVERAGE INDIVIOUAL DOSE-COMMITMENT HISTOGRAM FOR

\section{MONT ICELLO}

Oose Commitments (person-rem) from Waterborne Pathways

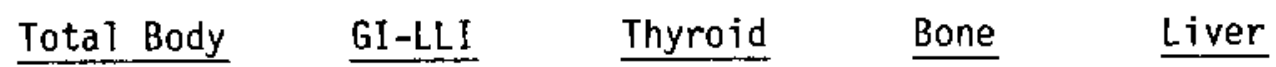

Infant

Child

Teen

Aduit

(No Waterborne Pathway Doses)

TOTAL

Oose Commitments (person-rem) from Airborne Pathways

Total Body GI-LLI Thyroid Bone Liver Lung

$\begin{array}{lllllll}\text { Infant } & 8.3 \mathrm{E}-04 & 1.0 \mathrm{E}-03 & 8.4 \mathrm{E}-03 & 1.2 \mathrm{E}-03 & 1.8 \mathrm{E}-03 & 6.3 \mathrm{E}-04 \\ \text { ChiTd } & 1.1 \mathrm{E}-02 & 6.9 \mathrm{E}-03 & 5.8 \mathrm{E}-02 & 1.7 \mathrm{E}-02 & 1.6 \mathrm{E}-02 & 8.1 \mathrm{E}-03 \\ \text { Teen } & 6.2 \mathrm{E}-03 & 5.5 \mathrm{E}-03 & 2.4 \mathrm{E}-02 & 7.2 \mathrm{E}-03 & 8.6 \mathrm{E}-03 & 6.0 \mathrm{E}-03 \\ \text { AduTt } & 3.2 \mathrm{E}-02 & 3.1 \mathrm{E}-02 & 8.9 \mathrm{E}-02 & 3.2 \mathrm{E}-02 & 3.7 \mathrm{E}-02 & 3.0 \mathrm{E}-02 \\ \text { TOTAL } & 4.9 \mathrm{E}-02 & 4.4 \mathrm{E}-02 & 1.8 \mathrm{E}-01 & 5.7 \mathrm{E}-02 & 6.3 \mathrm{E}-02 & 4.5 \mathrm{E}-02\end{array}$

Production/Consumption factors:

Produce: <1 Milk: 1.4 Meat: <1

Fraction of Population Receiving an Average Individual

Total-Bady Dose Commitment from Airborne Pathways

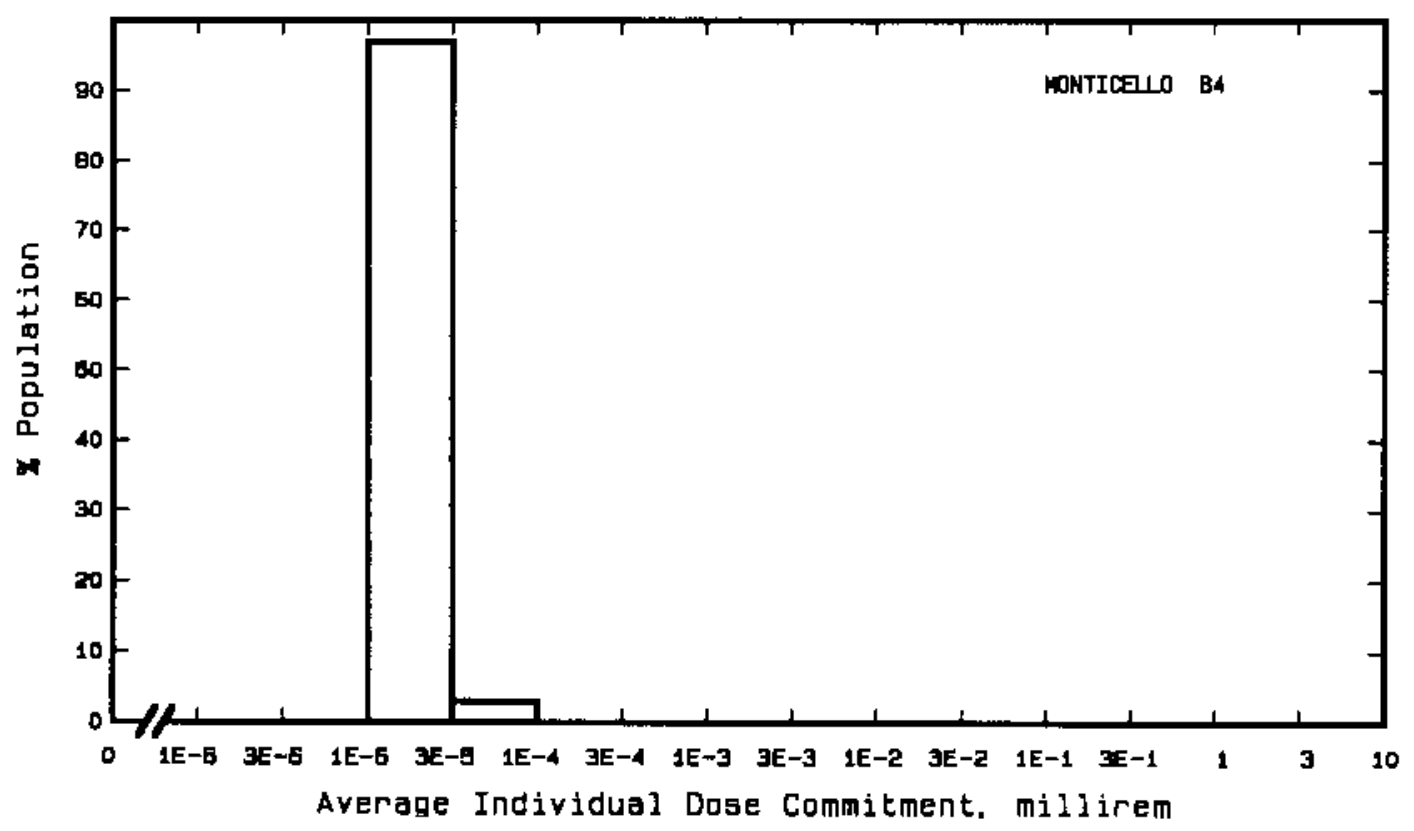


Site: NINE MILE POINT

OSWEGO, NEW YORK

Location: N $43.5222^{\circ}$ W $76.4100^{\circ}$

POPULATION DATA

Total Population Within 2-to-80-km Region: 8.5E5

Major Metropolitan Centers Within Region:

Center

Syracuse SMSA

Auburn

Watertown

Kingston

Oswego
Poputation

670,000

33,000

28,000

24,000

20,000
Location

$56 \mathrm{~km} \quad$ SSE

$67 \mathrm{~km} \quad \mathrm{SSW}$

$64 \mathrm{~km} \quad \mathrm{NE}$

$79 \mathrm{~km} \quad \mathrm{~N}$

$11 \mathrm{~km} \quad \mathrm{SW}$

SITE-SPECIFIC DATA - AIRBORNE PATHWAYS

Average Annual State Production

of Crops and Animal Products

In 80-km Radius Circle

Regiona? Productivity Factor:

Animal Grazing Factor:

Meteorology Period of Record: I JAN 74 - 3L DEC 75 Recovery: 97\%
Veg: $7.6 \mathrm{E} 7 \mathrm{kilogram}$

Milk: $7.0 \mathrm{E} 8$ liter

Meat: $3.3 E 7$ kilogram

0.7

0.5

SITE-SPECIFIC DATA - WATERBORNE PATHWAYS via LAKE ONTARIO

$\begin{array}{ll} & \begin{array}{l}\text { Average DiTution Flow } \\ \text { from Plant: } 480 \mathrm{ft}^{3} / \mathrm{s}\end{array} \\ \text { Drinking Water: } & \text { Exposed Poputation: (a) }\end{array}$

Fish:

Edible Harvest: (a)

(a) No Tiquid releases reported (Tichler, et al. 1987). 
POPULATION DOSE-COMMITMENT ESTIMATES AND

AVERAGE INDIVIDUAL DOSE-COMMITMENT HISTOGRAM FOR

\section{NINE MILE POINT}

Dose Commitments (person-rem) from Waterborne Pathways

Total Body GI-LLI Thyroid Bone Liver

Infant

Chiid

Teen

Adu?t

(No Waterborne Pathway Doses)

TOTAL

Dose Commitments (person-rem) from Airborne Pathways

$$
\text { Total Body GI-LLI Thyroid Bone Liver Lung }
$$

$\begin{array}{lllllll}\text { Infant } & 4.3 \mathrm{E}-04 & 4.1 \mathrm{E}-04 & 7.0 \mathrm{E}-03 & 4.5 \mathrm{E}-04 & 5.3 \mathrm{E}-04 & 4.4 \mathrm{E}-04 \\ \text { Chiid } & 5.2 \mathrm{E}-03 & 5.0 \mathrm{E}-03 & 4.9 \mathrm{E}-02 & 5.5 \mathrm{E}-03 & 5.9 \mathrm{E}-03 & 5.3 \mathrm{E}-03 \\ \text { Teen } & 3.5 \mathrm{E}-03 & 3.5 \mathrm{E}-03 & 2.0 \mathrm{E}-02 & 3.2 \mathrm{E}-03 & 3.7 \mathrm{E}-03 & 3.7 \mathrm{E}-03 \\ \text { Adult } & 2.0 \mathrm{E}-02 & 2.0 \mathrm{E}-02 & 7.4 \mathrm{E}-02 & 1.8 \mathrm{E}-02 & 2.1 \mathrm{E}-02 & 2.1 \mathrm{E}-02 \\ \text { TOTAL } & 2.9 \mathrm{E}-02 & 2.9 \mathrm{E}-02 & 1.5 \mathrm{E}-01 & 2.7 \mathrm{E}-02 & 3.1 \mathrm{E}-02 & 3.0 \mathrm{E}-02\end{array}$

Production/Consumption factors:

Produce: <1 Milk: $4.4 \quad$ Meat: <1

Fraction of Population Receiving an Average Individuad
Total-Body Dose Commitment from Airborne Pathways

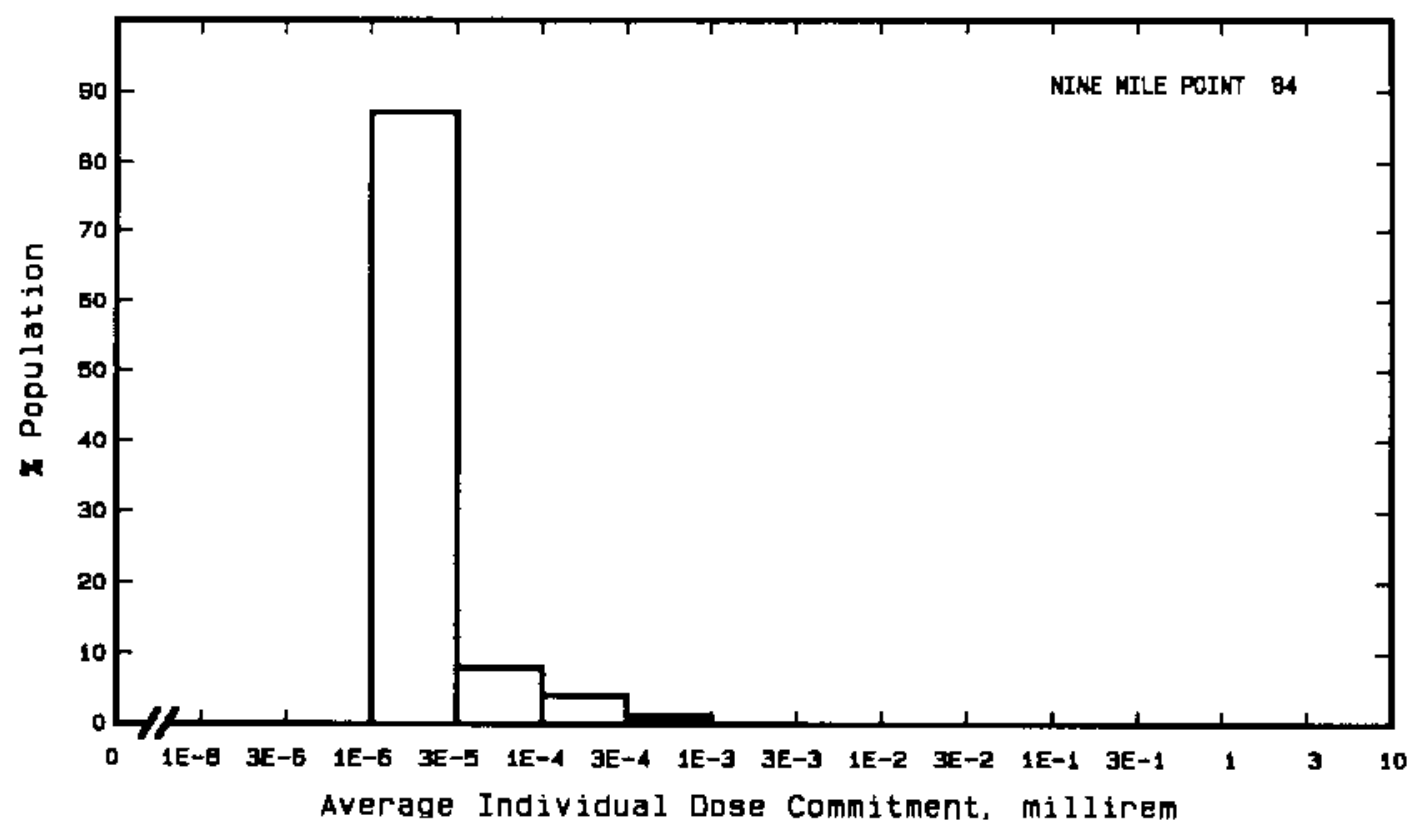


Site: NORTH ANNA

LOUISA COUNTY, VIRGINIA

Location: $\quad$ N $38.0608^{\circ}$ W $77.7906^{\circ}$

POPULATION DATA

Total Population Within 2-to-80-km Region: 1.1E6

Major Metropolitan Centers Within Region:

Center

Richmond SMSA

Charlottesville

Fredricksburg

Culpeper

Ashland
Population

650,000

42,000

16,000

6,800

4,800
Location

$66 \mathrm{~km} \quad$ SSE

$63 \mathrm{~km} \quad \mathrm{~W}$

$40 \mathrm{~km} \quad \mathrm{NE}$

$54 \mathrm{~km} \quad \mathrm{NNW}$

$41 \mathrm{~km} \quad \mathrm{SE}$

\section{SITE-SPECIFIC DATA - AIR8ORNE PATHWAYS}

Average Annual State Production

Of Crops and Anima T Products

In 80-km Radius Circle

Regional Productivity Factor:

Animal Grazing Factor:

Meteorology Period of Record: 1 APR 74 - 31 APR 75 Recovery: 99\%
Veg: $3.5 \mathrm{E} 7$ kilogram

MiTk: 1.5E8 Titer

Meat: $7.4 E 7$ ki logram

0.9

0.7

SITE-SPECIFIC DATA - WATERBORNE PATHWAYS via LAKE ANNA(a)

Average Dilution FTow from

Plant: $2,700 \mathrm{ft}^{3} / \mathrm{s}$

Drinking Water:

Exposed Population: None

Fish:

Edible Harvest: $7.3^{(\mathrm{b})}(\mathrm{kg} / \mathrm{yr}$

(a) Reconcentration of radionuclides in lake accounted for (FES 1973).

(b) Average individual consumption rates as given in FES (1973) were used in lieu of catch data. 
POPULATION DOSE-COMMITMENT ESTIMATES AND

AVERAGE INDIVIDUAL DOSE-COMMITMENT HISTOGRAM FOR

NORTH ANNA 1 AND 2

Dose Commitments (person-rem) from Waterborne Pathways

\begin{tabular}{|c|c|c|c|c|c|}
\hline & Total Body & GI-LLI & Thyroid & Bone & Liver \\
\hline $\begin{array}{l}\text { Infant } \\
\text { Child } \\
\text { Teen } \\
\text { Adult }\end{array}$ & $\begin{array}{l}0.0 \mathrm{E}+00 \\
1.8 \mathrm{E}+00 \\
3.6 \mathrm{E}+00 \\
3.9 \mathrm{E}+01\end{array}$ & $\begin{array}{l}0.0 \mathrm{E}+00 \\
6.6 \mathrm{E}-02 \\
1.3 \mathrm{E}-0 \mathrm{I} \\
1.0 \mathrm{E}+00\end{array}$ & $\begin{array}{l}0.0 \mathrm{E}+00 \\
1.8 \mathrm{E}-03 \\
1.4 \mathrm{E}-03 \\
9.6 \mathrm{E}-03\end{array}$ & $\begin{array}{l}0.0 \mathrm{E}+00 \\
9.5 \mathrm{E}+00 \\
5.7 \mathrm{E}+00 \\
3.3 \mathrm{E}+0 \mathrm{I}\end{array}$ & $\begin{array}{l}0.0 \mathrm{E}+00 \\
1.1 \mathrm{E}+01 \\
9.1 \mathrm{E}+00 \\
5.4 \mathrm{E}+01\end{array}$ \\
\hline TOTAL & 4. $4 \mathrm{E}+01$ & I. $2 \mathrm{E}+00$ & 1. $3 \mathrm{E}-02$ & $4.8 \mathrm{E}+01$ & $7.4 \mathrm{E}+01$ \\
\hline
\end{tabular}

Dose Commitments (person-rem) from Airborne Pathways

Total Body GI-LLI Thyroid Bone Liver Lung

$\begin{array}{lllllll}\text { Infant } & 2.6 \mathrm{E}-03 & 2.5 \mathrm{E}-03 & 7.4 \mathrm{E}-02 & 2.9 \mathrm{E}-03 & 3.0 \mathrm{E}-03 & 2.8 \mathrm{E}-03 \\ \text { Child } & 2.9 \mathrm{E}-02 & 2.8 \mathrm{E}-02 & 4.2 \mathrm{E}-01 & 3.1 \mathrm{E}-02 & 3.1 \mathrm{E}-02 & 3.2 \mathrm{E}-02 \\ \text { Teen } & 2.1 \mathrm{E}-02 & 2.1 \mathrm{E}-02 & 1.7 \mathrm{E}-01 & 2.1 \mathrm{E}-02 & 2.2 \mathrm{E}-02 & 2.5 \mathrm{E}-02 \\ \text { Adult } & 1.3 \mathrm{E}-01 & 1.2 \mathrm{E}-01 & 5.9 \mathrm{E}-01 & 1.3 \mathrm{E}-01 & 1.3 \mathrm{E}-01 & 1.4 \mathrm{E}-01 \\ \text { TOTAL } & 1.8 \mathrm{E}-01 & 1.8 \mathrm{E}-01 & 1.3 \mathrm{E}-00 & 1.8 \mathrm{E}-01 & 1.8 \mathrm{E}-01 & 2.0 \mathrm{E}-01\end{array}$

Production/Consumption factors:

Produce: <1 Milk: 1.0 Meat: <1

Fraction of Population Receiving an Average Individual

Total-Bady Dose Commitment from Airborne Pathways

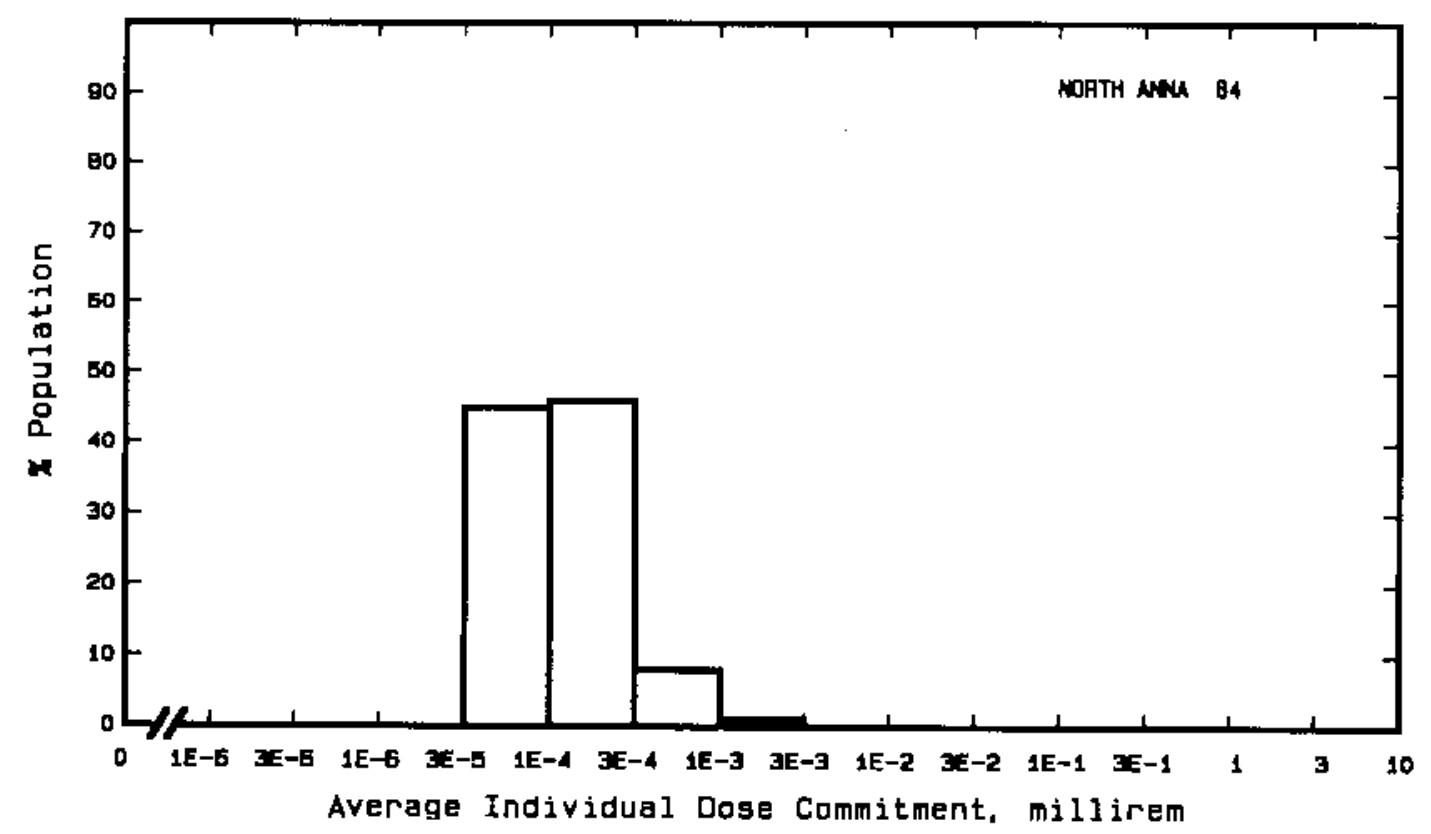


Site: OCONEE

OCONEE ZOUNTY, SOUTH CAROLINA

Location: N $34.7917^{\circ} \quad$ W $82.8985^{\circ}$

POPULATION DATA

Total Population Within 2-to-80-km Region: 9.3E5

Major Metropolitan Centers Within Region:

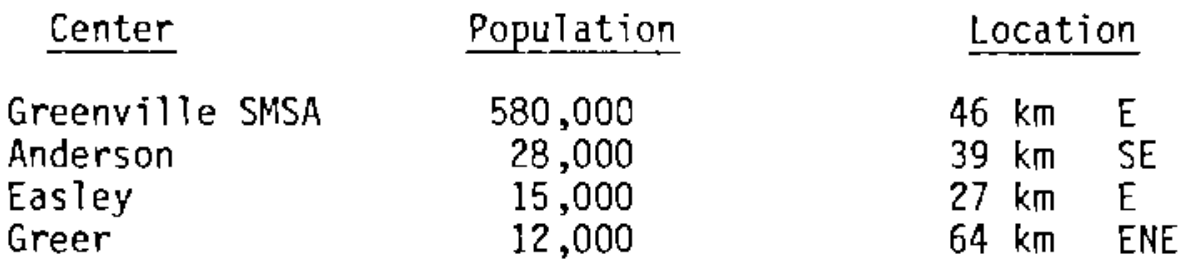

SITE-SPECIFIC DATA - AIRBORNE PATHWAYS

Average Annual State Production

of Crops and Animal Products

In $80-\mathrm{km}$ Radius Circle

Regional Productivity Factor:

Animal Grazing Factor:
Veg: 7.4E6 kilogram

Mi ik: $5.7 \mathrm{E} 7$ liter

Meat: $5.0 E 7$ kilogram

1

0.7

Meteorology Period of Record: I JAN 75 - 31 DEC 75 Recovery: 86\%

SITE-SPECIFIC DATA - WATERBORNE PATHWAYS via HARTWELL RES. ON KEOWEE RIVER

Average River Flow

at Site: $1,100 \mathrm{ft}^{3} / \mathrm{s}$

Drinking Water:

Exposed Population: 55,000

Dilution Factor: 1

Fish:

Edible Harvest: (a)

Dilution Factor: $0.01^{(b)}$

(a) No fish catch data given in FES, so generic consumption rates used (Table A-1).

(b) Ten percent of population obtain 10\% of their fish diet from Hartwell Reservoir according to FES (1972). 
POPULATION DOSE-COMMITMENT ESTIMATES AND

AVERAGE INOIVIDUAL OOSE-COMMITMENT HISTOGRAM FOR

OCONEE 1,2 ANO 3

Dose Commitments (person-rem) from Waterborne Pathways

\begin{tabular}{|c|c|c|c|c|c|c|}
\hline & Total Body & $G I-L$ & 11 & oid & Bone & Liver \\
\hline $\begin{array}{l}\text { Infant } \\
\text { Child } \\
\text { Teen } \\
\text { Aduit }\end{array}$ & $\begin{array}{l}5.7 \mathrm{E}-02 \\
6.9 \mathrm{E}-01 \\
3.3 \mathrm{E}-01 \\
3.0 \mathrm{E}+00\end{array}$ & $\begin{array}{l}5.71 \\
6.91 \\
3.41 \\
2.91\end{array}$ & & $\begin{array}{l}-01 \\
+00 \\
-01 \\
+00\end{array}$ & $\begin{array}{l}9.1 \mathrm{E}-03 \\
3.1 \mathrm{E}-01 \\
1.5 \mathrm{E}-01 \\
9.0 \mathrm{E}-01\end{array}$ & $\begin{array}{l}6.5 E-02 \\
9.5 E-01 \\
4.7 E-01 \\
3.4 E+00\end{array}$ \\
\hline TOTAL & 4. $I E+00$ & $4.0 \mathrm{E}$ & & $E+00$ & $1.4 \mathrm{E}+00$ & $4.9 E+00$ \\
\hline & Dose Commit & s lper & em) $f$ & irho & Pathways & \\
\hline & Total Body & GI-LII & Thyroid & Bone & Liver & Lung \\
\hline $\begin{array}{l}\text { Infant } \\
\text { Child } \\
\text { Teen } \\
\text { Adult }\end{array}$ & $\begin{array}{l}7.6 \mathrm{E}-03 \\
9.0 \mathrm{E}-02 \\
6.6 \mathrm{E}-02 \\
4.0 \mathrm{E}-01\end{array}$ & $\begin{array}{l}7.6 \mathrm{E}-03 \\
9.0 \mathrm{E}-02 \\
6.6 \mathrm{E}-02 \\
4.0 \mathrm{E}-01\end{array}$ & $\begin{array}{l}1.1 \mathrm{E}-02 \\
1.1 \mathrm{E}-01 \\
7.4 \mathrm{E}-02 \\
4.3 \mathrm{E}-01\end{array}$ & $\begin{array}{l}6.3 \mathrm{E}-03 \\
7.0 \mathrm{E}-02 \\
5.1 \mathrm{E}-02 \\
3.1 \mathrm{E}-01\end{array}$ & $\begin{array}{l}7.6 \mathrm{E}-03 \\
9.0 \mathrm{E}-02 \\
6.6 \mathrm{E}-02 \\
4.0 \mathrm{E}-01\end{array}$ & $\begin{array}{l}\text { 8.2E-03 } \\
9.9 \mathrm{E}-02 \\
7.7 \mathrm{E}-02 \\
4.3 \mathrm{E}-01\end{array}$ \\
\hline OTAL & $5.6 \mathrm{E}-01$ & $5.6 \mathrm{E}-01$ & $6.2 \mathrm{E}-0$ & $4.4 \mathrm{E}-01$ & $5.6 \mathrm{E}-01$ & $6.2 \mathrm{E}-01$ \\
\hline
\end{tabular}

Production/Consumption factors:

Produce: <1 Milk: <1 Meat: <1

Fraction of Population Receiving an Average Individual

Totel-Body Dose Commitment from Airborne Pathways

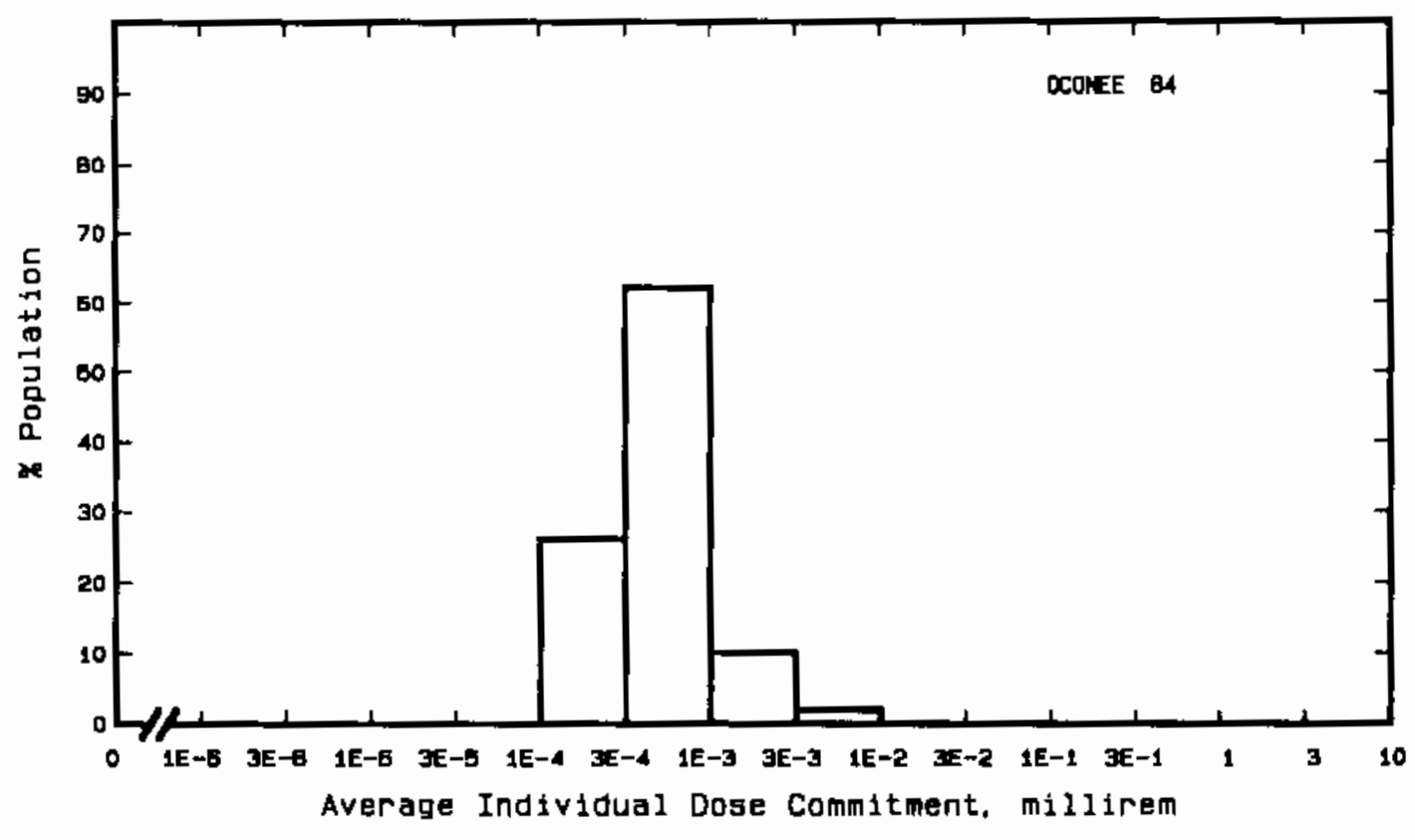


Site: OYSTER CREEK

OYSTEP CREEK, NEW JERSEY

Location: N $39.8142^{\circ}$ W $74.2064^{\circ}$

POPULATION DATA

Total Population Within 2-to-80-km Region: $3.5 \mathrm{E} 6$

Major Metropolitan Centers Within Region:

Center Population L Location

New Brunswick-Sayreville SMSA $600,000 \quad 77 \mathrm{~km} \quad \mathrm{~N}$

Long Branch-Asbury Park SMSA $\quad 500,000 \quad 57 \mathrm{~km}$ NNE

Trenton SMSA $\quad 310,000 \quad 66 \mathrm{~km} \quad \mathrm{NW}$

Atlantic City SMSA $\quad 190,000 \quad 55 \mathrm{~km} \quad$ SSW

Camden

85,000

$79 \mathrm{~km} \quad \mathrm{~W}$

SITE-SPECIFIC DATA - AIRBORNE PATHWAYS

Average Annual State Production

Veg: $7.4 \mathrm{E} 7$ kilogram

of Crops and Animal Products

In 80-km Radius Circle

MiTk: 2.7E8 liter

Meat: $2.4 \mathrm{E} 7$ kilogram

Regional Productivity Factor: $\quad 0.5$

Animal Grazing Factor: $\quad 0.6$

Meteorology Period of Record: 15 FEB 66 - 31 DEC 68 Recovery: 63\%

SITE-SPECIFIC DATA - WATERBORNE PATHWAYS via BARNEGAT BAY

Average DiTution Flow

from Plant: $600 \mathrm{ft}^{3} / \mathrm{s}$

Fish:

Edible Harvest: $2.1{ }^{(a)}(b \mathrm{~kg} / \mathrm{yr}$

Invertebrates:

Edible Harvest: $0.96^{(\mathrm{a})} \mathrm{kg} / \mathrm{yr}$

(a) Average individual consumption rate as given in the FES (1974) used in Tieu of catch data.

(b) $10 \%$ of seafood eaten assumed caught in bay waters diluted to $10 \%$ of that of discharge canal according to FES. 


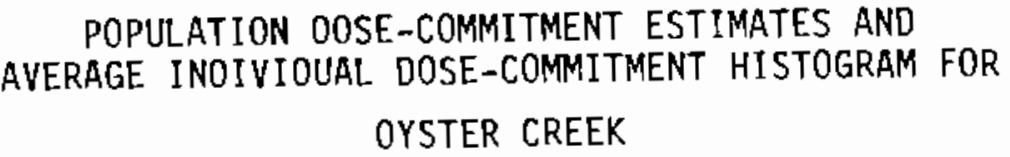

Oose Commitments (person-rem) from Waterborne Pathways

Total Body GI-LII Thyroid Bone Liver

$\begin{array}{llllll}\text { Infant } & 0.0 \mathrm{E}+00 & 0.0 \mathrm{E}+00 & 0.0 \mathrm{E}+00 & 0.0 \mathrm{E}+00 & 0.0 \mathrm{E}+00 \\ \text { Child } & 4.4 \mathrm{E}-04 & 7.9 \mathrm{E}-04 & 2.2 \mathrm{E}-05 & 1.0 \mathrm{E}-04 & 2.6 \mathrm{E}-04 \\ \text { Teen } & 3.3 \mathrm{E}-04 & 1.6 \mathrm{E}-03 & 2.0 \mathrm{E}-05 & 6.0 \mathrm{E}-05 & 2.2 \mathrm{E}-04 \\ \text { Adult } & 2.1 \mathrm{E}-03 & 1.4 \mathrm{E}-02 & 1.6 \mathrm{E}-04 & 3.5 \mathrm{E}-04 & 1.4 \mathrm{E}-03 \\ \text { TOTAL } & 2.9 \mathrm{E}-03 & 1.7 \mathrm{E}-02 & 2.0 \mathrm{E}-04 & 5.1 \mathrm{E}-04 & 1.9 \mathrm{E}-03\end{array}$

Dose Commitments (person-rem) from Airborne Pathways

\begin{tabular}{lccccccc} 
& Total Body & GI-LII & Thyroid & & Bone & Liver & Lung \\
\cline { 2 - 3 } Infant & $2.7 \mathrm{E}-02$ & $2.7 \mathrm{E}-02$ & $2.2 \mathrm{E}-01$ & $2.7 \mathrm{E}-02$ & $2.7 \mathrm{E}-02$ & $2.8 \mathrm{E}-02$ \\
Child & $3.0 \mathrm{E}-01$ & $3.0 \mathrm{E}-01$ & $1.8 \mathrm{E}+00$ & $3.0 \mathrm{E}-01$ & $3.0 \mathrm{E}-01$ & $3.1 \mathrm{E}-01$ \\
Teen & $2.2 \mathrm{E}-01$ & $2.2 \mathrm{E}-01$ & $9.2 \mathrm{E}-01$ & $2.2 \mathrm{E}-01$ & $2.2 \mathrm{E}-01$ & $2.4 \mathrm{E}-01$ \\
Adult & $1.3 \mathrm{E}+00$ & $1.3 \mathrm{E}+00$ & $4.2 \mathrm{E}+00$ & $1.3 \mathrm{E}+00$ & $1.3 \mathrm{E}+00$ & $1.4 \mathrm{E}+00$ \\
TOTAL & $1.8 \mathrm{E}+00$ & $1.9 \mathrm{E}+00$ & $7.1 \mathrm{E}+00$ & $1.9 \mathrm{E}+00$ & $1.9 \mathrm{E}+00$ & $2.0 \mathrm{E}+00$
\end{tabular}

Production/Consumption factors:

$$
\begin{array}{rlrl}
\text { Produce }: & <1 & \text { Milk: }<1 & \text { Meat: }<1 \\
& \text { Fraction of Population Feceiving an Average Individual } \\
& \text { Total-Body Dose Commitment from Airborne Pathways }
\end{array}
$$

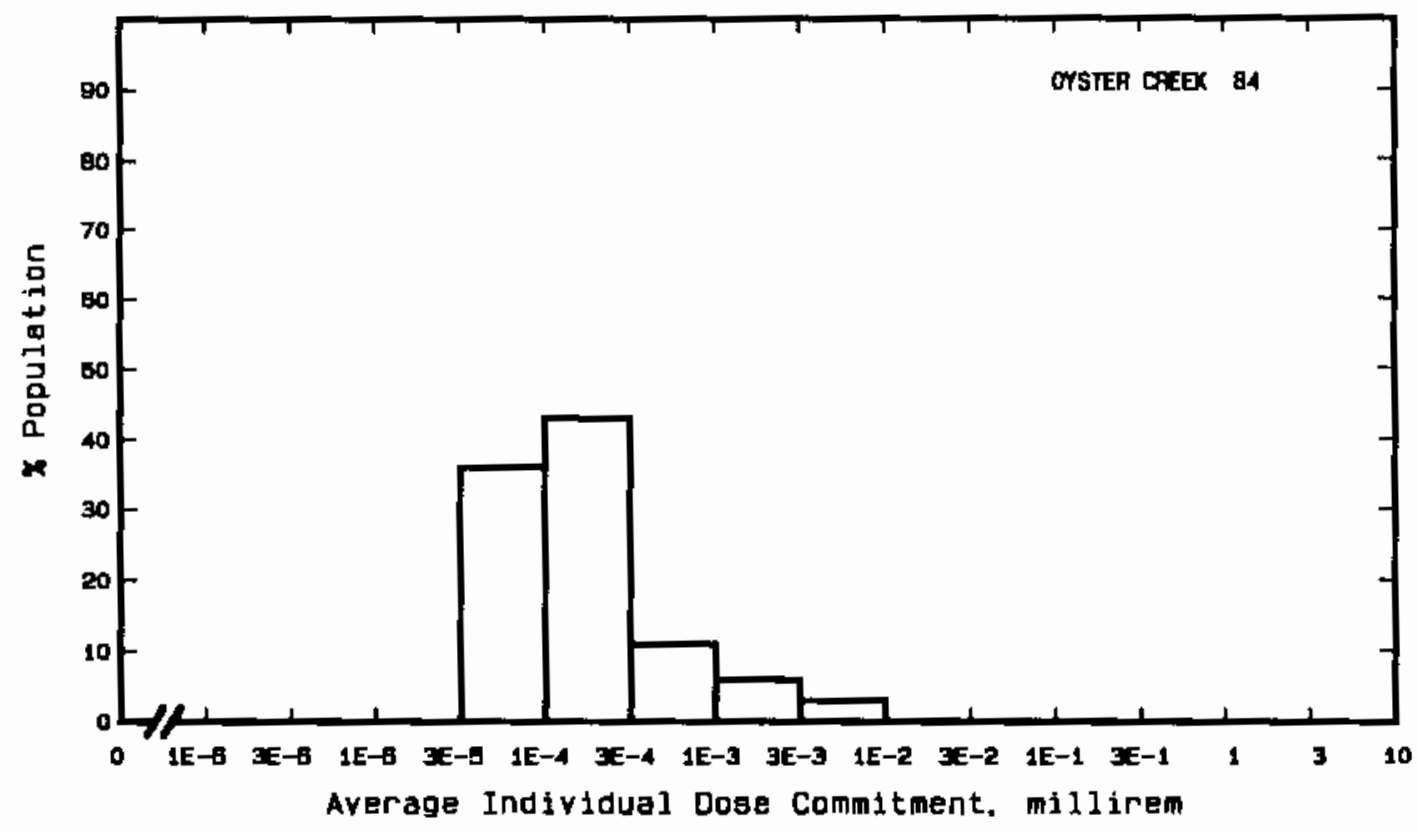


Site: PALISADES

Location: $\quad$ N $42.3222^{\circ}$ W $86.3153^{\circ}$

POPULATION DATA

Total Population Within 2-to-80-km Region: 1.0E6

Major Metropolitan Centers Within Region:

Center Population Location

Kalamazoo-Portage SMSA 280,000 $\quad 61 \mathrm{~km} \quad \mathrm{E}$

South Bend SMSA $280,000 \quad 72 \mathrm{~km} \quad \mathrm{~S}$

ETkhart SMSA $\quad 140,000 \quad 76 \mathrm{~km} \quad$ SSE

Holland $26,000 \quad 53 \mathrm{~km}$ NNE

Benton Harbor $\quad 15,000 \quad 25 \mathrm{~km} \quad \mathrm{SSW}$

SITE-SPECIFIC DATA - AIRBORNE PATHWAYS

Average Annual State Production

of Crops and Animal Products

In 80-km Radius Circle

Regional Productivity Factor:

Anima T Grazing Factor:

Meteorology Period of Record: I SEP 73 - 31 AUG 74 Recovery: 67\%
Veg: $6.8 \mathrm{E} 7 \mathrm{kiTogram}$

Milk: 2.9E8 liter

Meat: $4.5 E 7$ kilogram

0.6

0.5

SITE-SPECIFIC DATA - WATERBORNE PATHWAYS via LAKE MICHIGAN

Average Dilution Flow from

Plant: $110 \mathrm{ft}^{3} / \mathrm{s}$

Drinking Water:

Exposed Population: 50,909

Fish:

Edible Harvest: (b)

Dilution Factor: 0.001

(a) Drinking water dilution factor estimated by averaging diTution factor derived from FES (1972) suitably weighted for population.

(b) Average individual consumption rate of $2 \mathrm{C} \mathrm{g} / \mathrm{d}$ as given in FES used in lieu of catch data. 
POPULATION DOSE-COMMITMENT ESTIMATES AND

AVERAGE INDIVIDUAL DOSE-COMMITMENT HISTOGRAM FOR

\section{PALISADES}

Dose Commitments (person-rem) from Waterborne Pathways

\begin{tabular}{|c|c|c|c|c|c|}
\hline & TotaI Body & GI-LII & Thyroid & Bone & Liver \\
\hline $\begin{array}{l}\text { Infant } \\
\text { Child } \\
\text { Teen } \\
\text { Adult }\end{array}$ & $\begin{array}{l}1.0 \mathrm{E}-04 \\
6.1 \mathrm{E}-03 \\
9.9 \mathrm{E}-03 \\
1.1 \mathrm{E}-01\end{array}$ & $\begin{array}{l}9.8 \mathrm{E}-05 \\
1.5 \mathrm{E}-03 \\
1.1 \mathrm{E}-03 \\
9.4 \mathrm{E}-03\end{array}$ & $\begin{array}{l}9.6 \mathrm{E}-05 \\
1.1 \mathrm{E}-03 \\
4.5 \mathrm{E}-04 \\
3.9 \mathrm{E}-03\end{array}$ & $\begin{array}{l}2.6 \mathrm{E}-05 \\
2.9 \mathrm{E}-02 \\
1.7 \mathrm{E}-02 \\
9.8 \mathrm{E}-02\end{array}$ & $\begin{array}{l}1.3 \mathrm{E}-04 \\
3.1 \mathrm{E}-02 \\
2.6 \mathrm{E}-02 \\
1.5 \mathrm{E}-01\end{array}$ \\
\hline TOTAL & $1.2 \mathrm{E}-01$ & $1.2 E-02$ & $5.5 E-03$ & $1.4 \mathrm{E}-01$ & 2. $1 E-01$ \\
\hline
\end{tabular}

Dose Commitments (person-rem) from Airborne Pathways Total Body GI-LII Thyroid Bone Liver Lung

$\begin{array}{lllllll}\text { Infant } & 2.1 \mathrm{E}-05 & 2.0 \mathrm{E}-05 & 3.8 \mathrm{E}-04 & 7.2 \mathrm{E}-06 & 2.3 \mathrm{E}-05 & 2.1 \mathrm{E}-05 \\ \text { Child } & 2.7 \mathrm{E}-04 & 2.7 \mathrm{E}-04 & 2.5 \mathrm{E}-03 & 7.2 \mathrm{E}-05 & 2.9 \mathrm{E}-04 & 2.8 \mathrm{E}-04 \\ \text { Teen } & 1.7 \mathrm{E}-04 & 1.7 \mathrm{E}-04 & 1.0 \mathrm{E}-03 & 4.3 \mathrm{E}-05 & 1.8 \mathrm{E}-04 & 1.8 \mathrm{E}-04 \\ \text { Aduit } & 9.2 \mathrm{E}-04 & 9.1 \mathrm{E}-04 & 3.9 \mathrm{E}-03 & 2.4 \mathrm{E}-04 & 9.3 \mathrm{E}-04 & 9.5 \mathrm{E}-04 \\ \text { TOTAL } & 1.4 \mathrm{E}-03 & 1.4 \mathrm{E}-03 & 7.8 \mathrm{E}-03 & 3.6 \mathrm{E}-04 & 1.4 \mathrm{E}-03 & 1.4 \mathrm{E}-03\end{array}$

Production/Consumption factors:

Produce: <1 Milk: 1.3 Meat: <1

Fraction of Population Receiving an Average Individual Total-Body Dose Commitment from Airborne Pathways

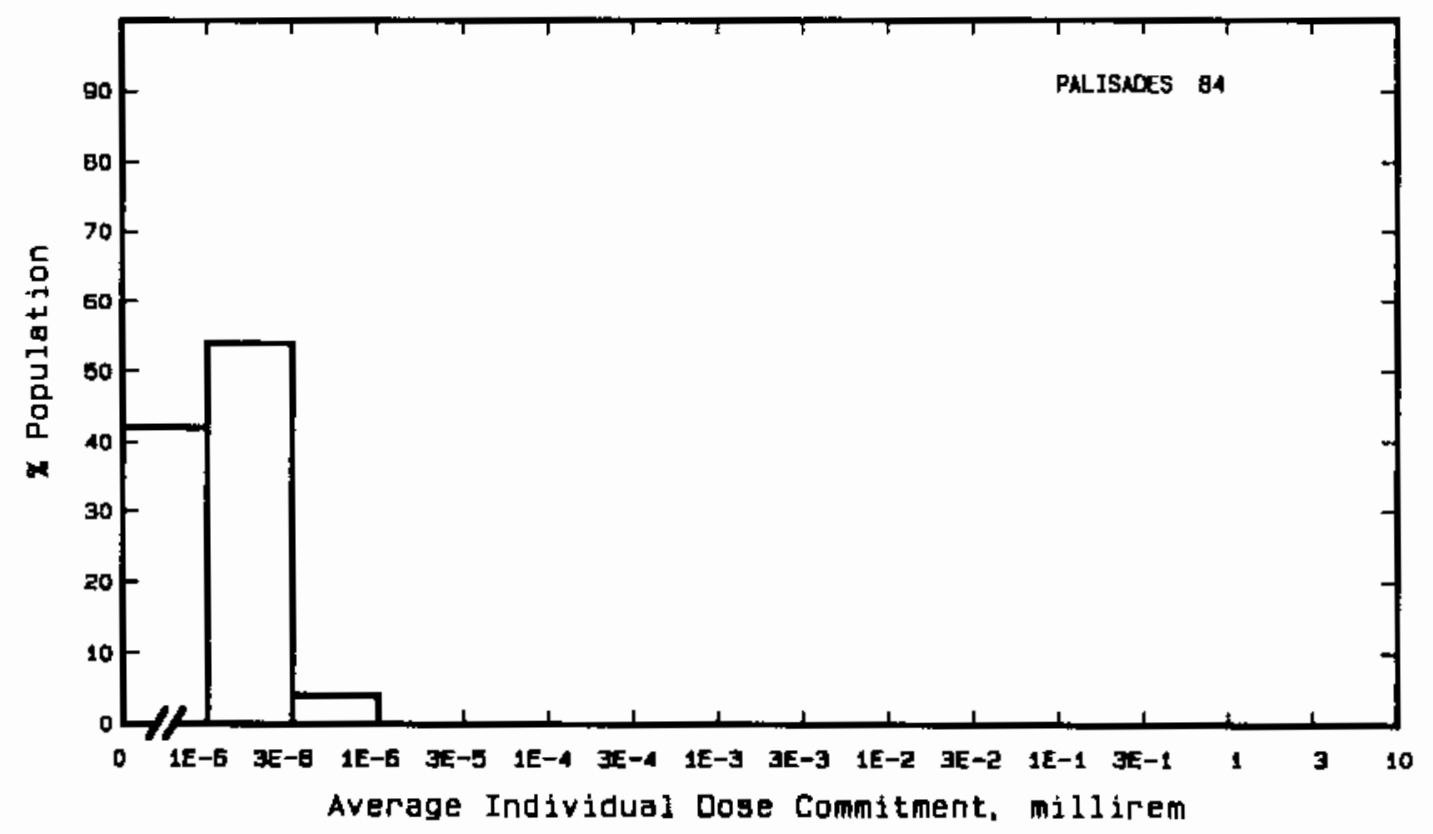


Site: PEACH BOTTOM

YORK COUNTY, PENNSYLVANIA

Location: N $39.7589^{\circ}$ W $76.2692^{\circ}$

POPULATION DATA

Total Population Within 2-to-80-km Region: 4.3E6

Major Metropolitan Centers Within Region:

\begin{tabular}{|c|c|c|c|}
\hline Center & Population & \multicolumn{2}{|c|}{ Location } \\
\hline Baltimore SMSA & $2,200,000$ & $6 \mathrm{D} \mathrm{km}$ & \\
\hline Harrisburg SMSA & 450,000 & $77 \mathrm{~km}$ & \\
\hline Wilmington SMSA & 400,000 & $62 \mathrm{~km}$ & \\
\hline York SMSA & 380,000 & $45 \mathrm{~km}$ & $\mathrm{NW}$ \\
\hline Lancaster SMSA & 360,000 & $31 \mathrm{~km}$ & $\mathrm{~N}$ \\
\hline
\end{tabular}

SITE-SPECIFIC DATA - AIRBORNE PATHWAYS

Average Annual State Production

of Crops and Animal Products

In $80-\mathrm{km}$ Radius Circle

Regional Productivity Factor:

Animal Grazing Factor:

Meteorology Period of Record: 1 AUG $67-31$ JUL 71 Recovery: $72 \%$
Veg: $5.3 \mathrm{E} 7 \mathrm{kiTogram}$

Milk: 5.3E8 Titer

Meat: $5.4 \mathrm{E} 7$ kilogram

0.95

0.6

SITE-SPECIFIC DATA - WATERBORNE PATHWAYS via SUSQUEHANNA RIVER

Average River Flow

at Site: $36,000 \mathrm{ft}^{3} / \mathrm{s}$

Drinking Water:

Exposed Population: 2.2E6

Dilution Fáctor: 1

Fish:

Edible Harvest: (a)

Dilution Factor: 0.001

(a) No fish catch data given in FES (1974), thus generic consumption rates used (Table A-1).

(b) One percent of people obtain $10 \%$ of their fish diet from river downstream from plant according to FES. 


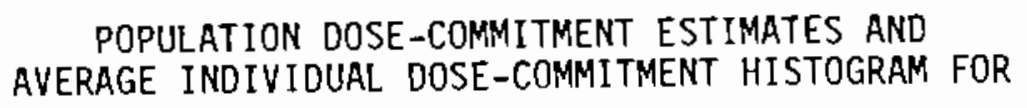

PEACH BOTTOM 2 AND 3

Dose Commitments (person-rem) from Waterborne Pathways

\begin{tabular}{|c|c|c|c|c|c|}
\hline & Total Body & GI-LII & Thyroid & Bone & $\underline{\text { Liver }}$ \\
\hline $\begin{array}{l}\text { Infant } \\
\text { Child } \\
\text { Teen } \\
\text { Adult }\end{array}$ & $\begin{array}{l}9.6 \mathrm{E}-03 \\
1.4 \mathrm{E}-01 \\
8.4 \mathrm{E}-02 \\
8.7 \mathrm{E}-01\end{array}$ & $\begin{array}{l}5.2 E-03 \\
5.0 E-02 \\
3.2 E-02 \\
2.9 E-01\end{array}$ & $\begin{array}{l}3.3 \mathrm{E}-0 \mathrm{I} \\
2.3 \mathrm{E}+00 \\
7.1 \mathrm{E}-01 \\
5.0 \mathrm{E}+00\end{array}$ & $\begin{array}{l}5.6 \mathrm{E}-02 \\
6.1 \mathrm{E}-0 \mathrm{I} \\
1.7 \mathrm{E}-01 \\
1 . \mathrm{IE}+00\end{array}$ & $\begin{array}{l}4.7 \mathrm{E}-02 \\
4.4 \mathrm{E}-01 \\
1.7 \mathrm{E}-01 \\
1.1 \mathrm{E}+00\end{array}$ \\
\hline TOTAL & $1.1 \mathrm{E}+00$ & $3.7 E-01$ & $8.4 E+00$ & $1.9 E+00$ & $1.7 \mathrm{E}+00$ \\
\hline
\end{tabular}

Dose Commitments (person-rem) from Airborne Pathways Total Body GI-LII Thyroid one Liver Lung

$\begin{array}{lllllll}\text { Infant } & 1.2 \mathrm{E}-01 & 1.2 \mathrm{E}-01 & 3.2 \mathrm{E}-01 & 1.2 \mathrm{E}-0 \mathrm{I} & 1.2 \mathrm{E}-01 & 1.3 \mathrm{E}-0 \mathrm{I} \\ \text { Child } & 1.4 \mathrm{E}+00 & 1.4 \mathrm{E}+00 & 2.7 \mathrm{E}+00 & 1.4 \mathrm{E}+00 & 1.4 \mathrm{E}+00 & 1.5 \mathrm{E}+00 \\ \text { Teen } & 9.9 \mathrm{E}-01 & 9.9 \mathrm{E}-01 & 1.6 \mathrm{E}+00 & 9.9 \mathrm{E}-01 & 9.9 \mathrm{E}-01 & 1.2 \mathrm{E}+00 \\ \text { Adu1t } & 6.0 \mathrm{E}+00 & 6.0 \mathrm{E}+00 & 8.2 \mathrm{E}+00 & 6.0 \mathrm{E}+00 & 6.0 \mathrm{E}+00 & 6.6 \mathrm{E}+00 \\ \text { TOTAL } & 8.5 \mathrm{E}+00 & 8.5 \mathrm{E}+00 & 1.3 \mathrm{E}+01 & 8.5 \mathrm{E}+00 & 8.5 \mathrm{E}+00 & 9.4 \mathrm{E}+00\end{array}$

Production/Consumption factors:

Produce: $<1 \quad$ Milk: $<1 \quad$ Meat: $<1$

Fraction of Population Receiving an Average Individual

Tota1-Body Dose Commitment from Airborne Pathways

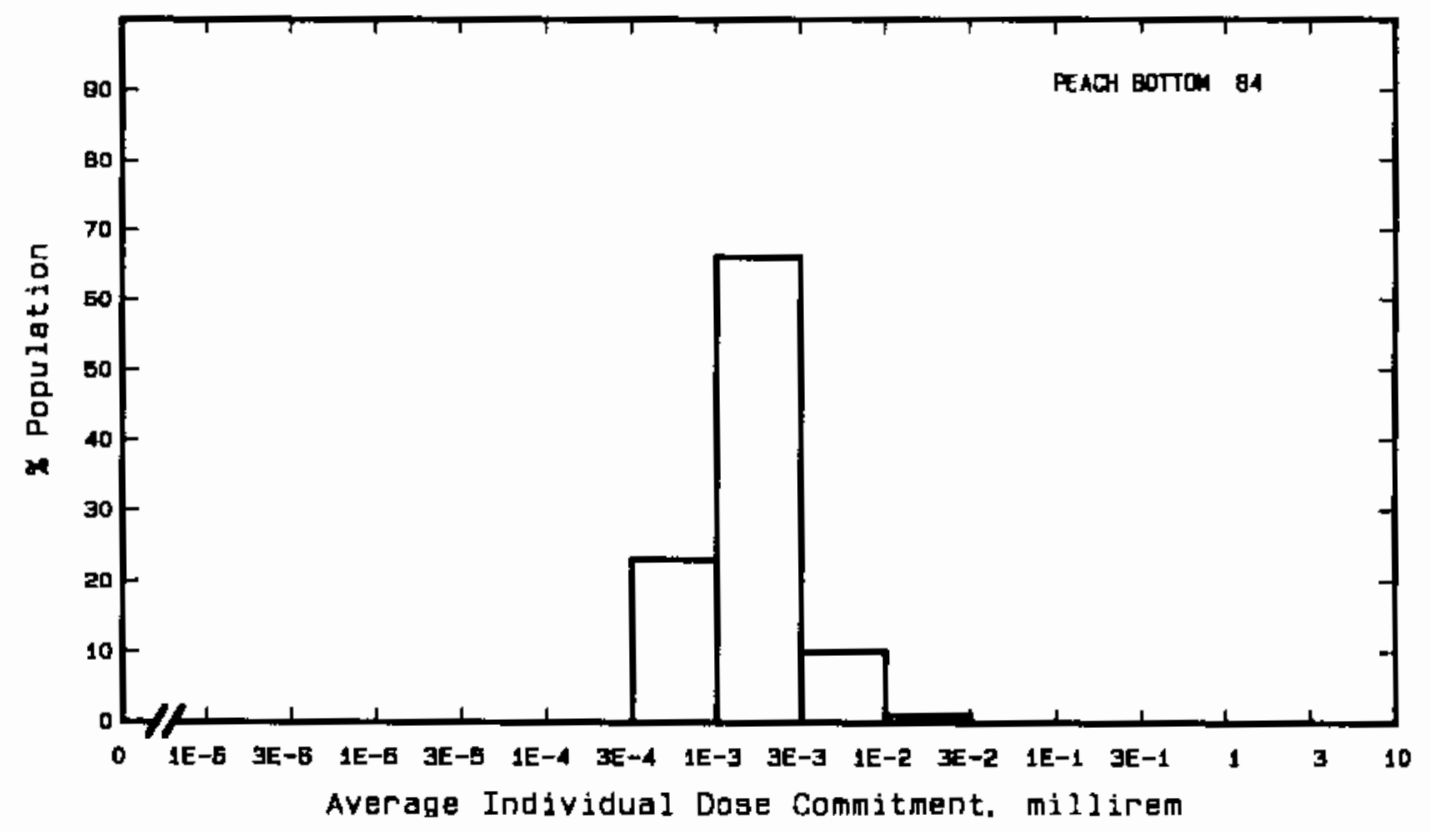


Site: PILGRIM

PLYMOITTH, MASSACHUSETTS

Location: N $41.9444^{\circ} \quad$ W $70.5794^{\circ}$

POPULATION DATA

Total Population Within 2-to-80-km Region: $4.3 \mathrm{E} 6$

Major Metropolitan Centers Within Region:

\begin{tabular}{lrrrr}
\multicolumn{1}{c}{ Center } & Population & \multicolumn{2}{c}{ Location } \\
\cline { 2 - 4 } & & & \\
Boston SMSA & $2,800.000$ & $61 \mathrm{~km}$ & NW \\
Providence-Warwick-Pawtucket SMSA & 820.000 & $70 \mathrm{~km}$ & W \\
New Bedford SMSA & 170.000 & $45 \mathrm{~km}$ & SSW \\
Brockton SMSA & 170.000 & $40 \mathrm{~km}$ & WNW \\
Fa11 River SMSA & 150.000 & $55 \mathrm{~km}$ & SW
\end{tabular}

SITE-SPECIFIC DATA - AIRBORNE PATHWAYS

Average Annuat State Production

Veg: $2.0 \mathrm{E} 7$ kilogram

of Crops and AnimaT Products

Mi1k: 2.6E8 liter

In 80-km Radius Circle

Meat: $1.6 \mathrm{E} 7$ kilogram

Regional Productivity Factor:

0.3

Animal Grazing Factor:

0.6

Meteorology Period of Record: I MAY 74 - 30 APR 75 Recovery: 93\%

SITE-SPECIFIC DATA - WATERBORNE PATHWAYS via CAPE COD BAY

Average Dilution Flow

from Plant: $55 \mathrm{ft}^{3} / \mathrm{s}$

Fish:

Edible Harvest: $2.6 \mathrm{E} 4 \mathrm{~kg} / \mathrm{yr}$

Dilution Fector: 0.001

Invertebrates:

Edible Harvest: $3.1 E 4 \mathrm{~kg} / \mathrm{yr}$

Dilution Factor: 0.002 


\section{POPULATION DOSE-COMMITMENT ESTIMATES AND \\ AVERAGE INDIVIDUAL OOSE-COMMITMENT HISTOGRAM FOR}

PILGRIM

Oose Commitments (person-rem) from Waterborne Pathways

\begin{tabular}{|c|c|c|c|c|c|}
\hline & Total Body & GI-LII & Thyroid & Bone & Liver \\
\hline $\begin{array}{l}\text { Infant } \\
\text { Child } \\
\text { Teen } \\
\text { Adult }\end{array}$ & $\begin{array}{l}0.0 \mathrm{E}+00 \\
6.7 \mathrm{E}-03 \\
4.5 \mathrm{E}-03 \\
2.9 \mathrm{E}-02\end{array}$ & $\begin{array}{l}0.0 \mathrm{E}+00 \\
7.1 \mathrm{E}-03 \\
1.4 \mathrm{E}-02 \\
1.2 \mathrm{E}-01\end{array}$ & $\begin{array}{l}0.0 \mathrm{E}+00 \\
3.4 \mathrm{E}-07 \\
2.9 \mathrm{E}-07 \\
2.3 \mathrm{E}-06\end{array}$ & $\begin{array}{l}0.0 \mathrm{E}+00 \\
1.8 \mathrm{E}-02 \\
9.8 \mathrm{E}-03 \\
5.8 \mathrm{E}-02\end{array}$ & $\begin{array}{l}0.0 \mathrm{E}+00 \\
1.3 \mathrm{E}-02 \\
9.8 \mathrm{E}-03 \\
5.8 \mathrm{E}-02\end{array}$ \\
\hline TOTAL & $4,0 E-02$ & $1.4 \mathrm{E}-01$ & $2.9 \mathrm{E}-06$ & $8.5 E-02$ & $8.0 E-02$ \\
\hline
\end{tabular}

Dose Commitments (person-rem) from Airborne Pathways Total Body GI-LII Thyroid Bone Liver Lung

$\begin{array}{lllllll}\text { Infant } & 1.5 \mathrm{E}-04 & 1.5 \mathrm{E}-04 & 2.1 \mathrm{E}-04 & 1.7 \mathrm{E}-04 & 1.8 \mathrm{E}-04 & 2.2 \mathrm{E}-04 \\ \text { Child } & 1.7 \mathrm{E}-03 & 1.7 \mathrm{E}-03 & 2.2 \mathrm{E}-03 & 1.8 \mathrm{E}-03 & 1.9 \mathrm{E}-03 & 2.9 \mathrm{E}-03 \\ \text { Teen } & 1.3 \mathrm{E}-03 & 1.3 \mathrm{E}-03 & 1.5 \mathrm{E}-03 & 1.2 \mathrm{E}-03 & 1.3 \mathrm{E}-03 & 2.3 \mathrm{E}-03 \\ \text { Adult } & 7.7 \mathrm{E}-03 & 7.8 \mathrm{E}-03 & 8.6 \mathrm{E}-03 & 7.3 \mathrm{E}-03 & 7.7 \mathrm{E}-03 & 1.2 \mathrm{E}-02 \\ \text { TOTAL } & 1.1 \mathrm{E}-02 & 1.1 \mathrm{E}-02 & 1.3 \mathrm{E}-02 & 1.1 \mathrm{E}-02 & 1.1 \mathrm{E}-02 & 1.7 \mathrm{E}-02\end{array}$

Production/Consumption factors:

Produce: $<1 \quad$ Milk: <1 Meat: <1

Fraction of Population Receiving an Average Individual

Totol-Body Dose Commitment from Airborne Pathways

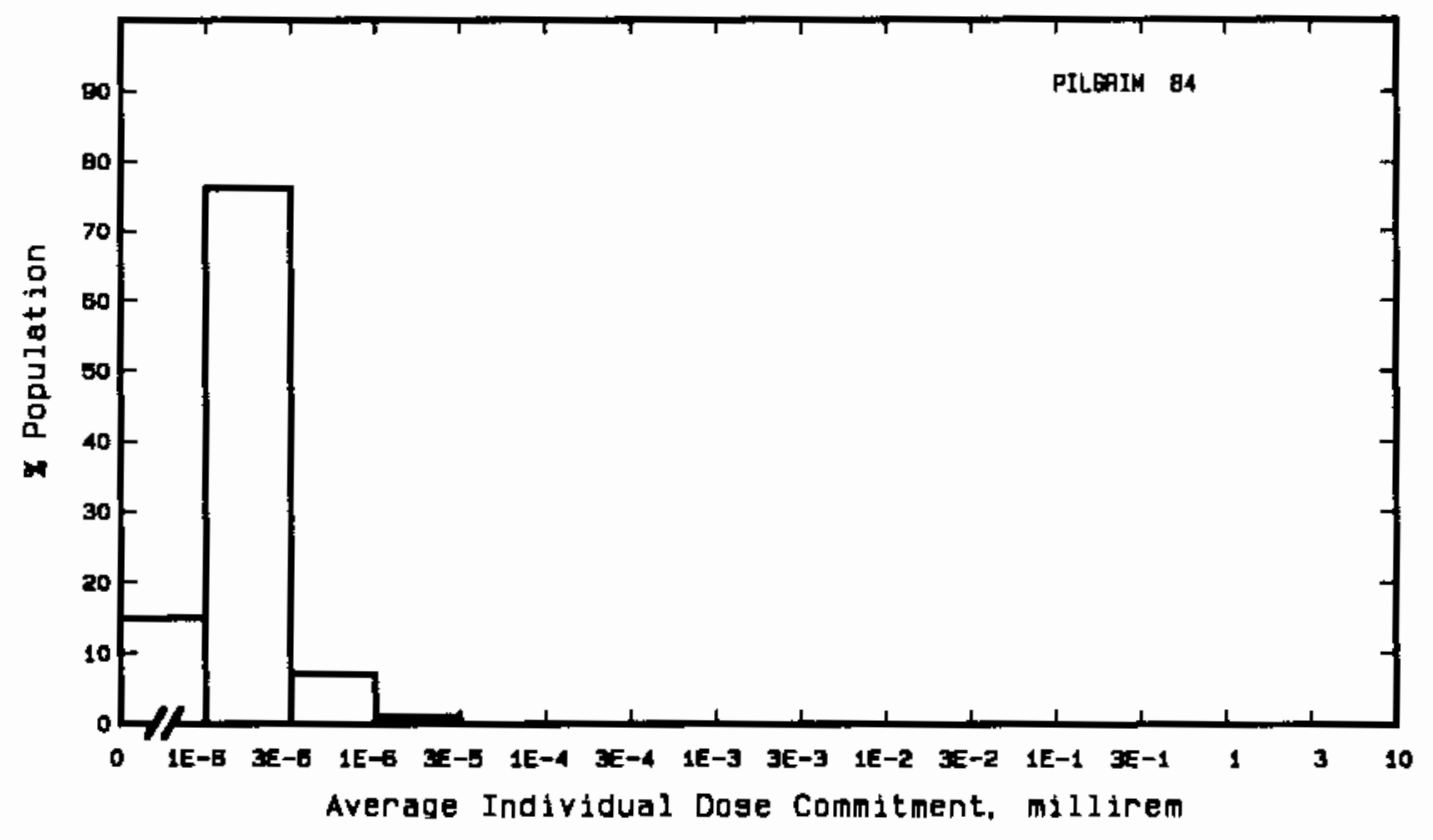


Site: POINT BEACH

MANITOWOC COUNTY, WISCONSIN
Location:
N $44.2808^{\circ}$
W $87.5361^{\circ}$

POPULATION DATA

Total Population Within 2-to-80-km Region: 6.2E5

Major Metropolitan Centers Within Region:

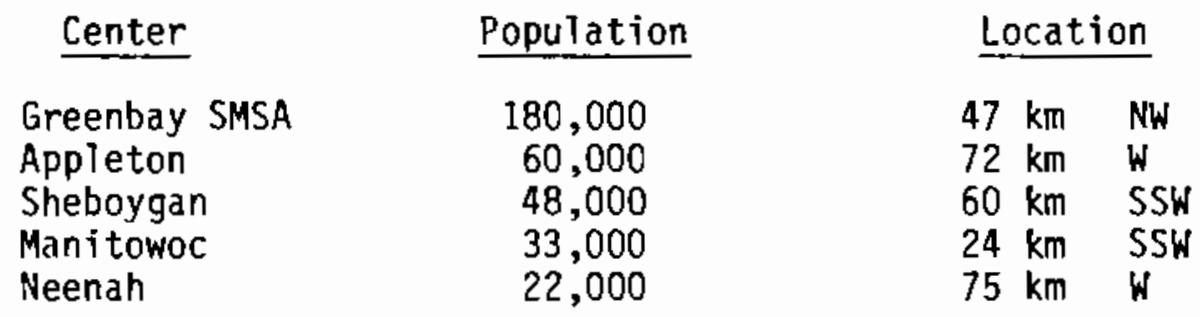

SITE-SPECIFIC DATA - AIRBORNE PATHWAYS

Average Annual State Production

of Crops and Animal Products

In $80-\mathrm{km}$ Radius Circle

Regional Productivity Factor:

Animal Grazing Factor:
Veg: $7.2 E 7$ kilogram

Milk: 1.2E9 liter

Meat: 1.0E8 kilogram

0.5

0.5

Meteorology Period of Record: 19 APR 67 - 18 APR 69 Recovery: 83\%

SITE-SPECIFIC DATA - WATERBORNE PATHWAYS via LAKE MICHIGAN

Average Dilution Flow from Plant: $650 \mathrm{ft}^{3} / \mathrm{s}$

Drinking Water:

Exposed Population: 260 eg90

Dilution Factor: $2.6 \mathrm{E}-3$ (

Fish:

Edible Harvest: $6.7 \mathrm{E} 4(\mathrm{~b}) \mathrm{kg} / \mathrm{yr}$
Oilution Factor: $0.013(\mathrm{c})$

(a) Drinking water dilution factor estimated by averaging dilution factor derived from FES (1972), suitably weighted for population.

(b) Includes both sport and commercial fish catch from FES.

(c) Dilution factor estimated by averaging sport and commercial dilution factor derived from FES. 
POPULATION DOSE-COMMITMENT ESTIMATES AND
AVERAGE INDIVIDUAL DOSE-COMMITMENT HISTOGRAM FOR

POINT BEACH 1 AND 2

Dose Commitments (person-rem) from Waterborne Pathways

Total Body GI-LII Thyroid Bone Liver

$\begin{array}{llllll}\text { Infant } & 2.2 \mathrm{E}-03 & 2.3 \mathrm{E}-03 & 2.1 \mathrm{E}-03 & 2.9 \mathrm{E}-04 & 2.3 \mathrm{E}-03 \\ \text { Child } & 2.7 \mathrm{E}-02 & 4.0 \mathrm{E}-02 & 2.3 \mathrm{E}-02 & 1.3 \mathrm{E}-02 & 3.6 \mathrm{E}-02 \\ \text { Teen } & 1.3 \mathrm{E}-02 & 3.8 \mathrm{E}-02 & 8.5 \mathrm{E}-03 & 6.5 \mathrm{E}-03 & 1.9 \mathrm{E}-02 \\ \text { Aduit } & 1.2 \mathrm{E}-01 & 3.3 \mathrm{E}-01 & 7.2 \mathrm{E}-02 & 3.8 \mathrm{E}-02 & 1.3 \mathrm{E}-01 \\ \text { TOTAL } & 1.6 \mathrm{E}-01 & 4.1 \mathrm{E}-01 & 1.0 \mathrm{E}-01 & 5.7 \mathrm{E}-02 & 1.9 \mathrm{E}-01\end{array}$

Dose Commitments (person-rem) from Airborne Pathways

\begin{tabular}{lccccccc} 
& Total Body & GI-LII & Thyroid & Bone & liver & Lung \\
\cline { 2 - 3 } Infant & $9.5 \mathrm{E}-04$ & $9.5 \mathrm{E}-04$ & $1.1 \mathrm{E}-03$ & $4.2 \mathrm{E}-05$ & $9.6 \mathrm{E}-04$ & $9.5 \mathrm{E}-04$ \\
Child & $1.5 \mathrm{E}-02$ & $1.5 \mathrm{E}-02$ & $1.6 \mathrm{E}-02$ & $4.6 \mathrm{E}-04$ & $1.5 \mathrm{E}-02$ & $1.5 \mathrm{E}-02$ \\
Teen & $9.1 \mathrm{E}-03$ & $9.1 \mathrm{E}-03$ & $9.6 \mathrm{E}-03$ & $3.2 \mathrm{E}-04$ & $9.1 \mathrm{E}-03$ & $9.1 \mathrm{E}-03$ \\
Adult & $5.0 \mathrm{E}-02$ & $5.0 \mathrm{E}-02$ & $5.2 \mathrm{E}-02$ & $1.9 \mathrm{E}-03$ & $5.0 \mathrm{E}-02$ & $5.0 \mathrm{E}-02$ \\
TOTAL & $7.5 \mathrm{E}-02$ & $7.5 \mathrm{E}-02$ & $7.8 \mathrm{E}-02$ & $2.7 \mathrm{E}-03$ & $7.5 \mathrm{E}-02$ & $7.5 \mathrm{E}-02$
\end{tabular}

Production/Consumption factors:

Produce: <1 Milk: 7.2 Meat: 1.0

Fraction of Population Receiving an Average Indivioual

Total-Body Dose Commitment from Airborne Pathways

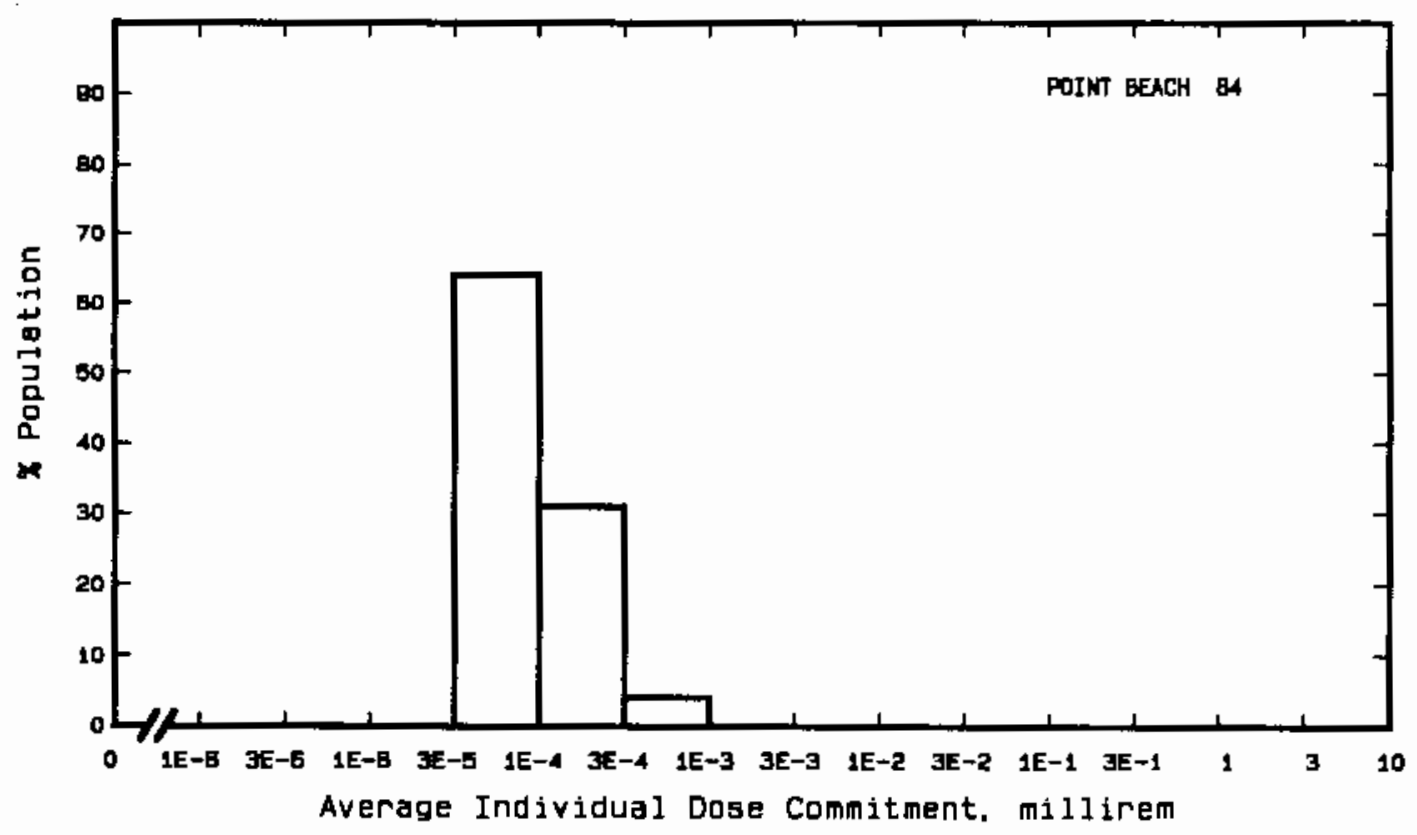


Site: PRAIRIE ISLAND

Location: N $44.6219^{\circ} \quad$ W $92.6331^{\circ}$

POPULATION DATA

Total Population Within 2-to-80-km Region: 2.2E6

Major Metropolitan Centers Within Region:

Center Population Location

Minneapolis-St. Paul SMSA $2,100,000 \quad 63 \mathrm{~km} \quad \mathrm{NW}$

Rochester SMSA $\quad 94,000 \quad 68 \mathrm{~km}$ SSE

Owatonna $\quad 19,000 \quad 77 \mathrm{~km} \quad \mathrm{SW}$

FaribauTt $\quad 16,000 \quad 63 \mathrm{~km} \quad \mathrm{SW}$

Redwing $14,000 \quad 10 \mathrm{~km} \quad \mathrm{SE}$

SITE-SPECIFIC OATA - AIRBORNE PATHWAYS

Average Annual State Production

of Crops and Animal Products

In $80-\mathrm{km}$ Radius Circle

Regional Productivity Factor:

Animal Grazing Factor:

Meteorology Period of Record: 22 MAR $74-21$ MAR 75 Recovery: 65\%
Veg: $1.2 \mathrm{E} 8 \mathrm{kilogram}$

MiTk: 4.0E8 Titer

Meat: $1.0 \mathrm{E} 8$ kilogram

1

0.5

SITE-SPECIFIC OATA - WATERBORNE PATHWAYS via MISSISSIPPI RIVER

Average River Flow

at Site: $15,000 \mathrm{ft}^{3} / \mathrm{s}$

Orinking Water:

Exposed Population: None

Fish:

Edible Harvest: $6.8 \mathrm{E} 5 \mathrm{~kg} / \mathrm{yr}$

DiTution Factor: 1 
POPULATION DOSE-COMMITMENT ESTIMATES AND

AVERAGE INOIVIDUAL DOSE-COMMITMENT HISTOGRAM FOR

PRAIRIE ISLAND 1 AND 2

Dose Commitments (person-rem) from Waterborne Pathways

\begin{tabular}{|c|c|c|c|c|c|}
\hline & Total Body & GI-LII & Thyroid & Bone & Liver \\
\hline $\begin{array}{l}\text { Infant } \\
\text { Child } \\
\text { Teen } \\
\text { Adult }\end{array}$ & $\begin{array}{l}0.0 \mathrm{E}+00 \\
7.8 \mathrm{E}-04 \\
1.1 \mathrm{E}-03 \\
1.1 \mathrm{E}-02\end{array}$ & $\begin{array}{l}0.0 \mathrm{E}+00 \\
5.2 \mathrm{E}-04 \\
6.6 \mathrm{E}-04 \\
5.5 \mathrm{E}-03\end{array}$ & $\begin{array}{l}0.0 \mathrm{E}+00 \\
5.7 \mathrm{E}-04 \\
4.7 \mathrm{E}-04 \\
3.6 \mathrm{E}-03\end{array}$ & $\begin{array}{l}0.0 \mathrm{E}+00 \\
1.9 \mathrm{E}-03 \\
1.1 \mathrm{E}-03 \\
6.5 \mathrm{E}-03\end{array}$ & $\begin{array}{l}0.0 \mathrm{E}+00 \\
2.6 \mathrm{E}-03 \\
2.3 \mathrm{E}-03 \\
1.4 \mathrm{E}-02\end{array}$ \\
\hline TOTAL & 1. $3 \mathrm{E}-02$ & $6.7 \mathrm{E}-03$ & 4.6E-03 & $9.5 E-03$ & $1.9 \mathrm{E}-02$ \\
\hline
\end{tabular}

Dose Cormmitments (person-rem) from Airborne Pathways

Total Body GI-LII Thyroid Bone Liver Lung

$\begin{array}{lllllll}\text { Infant } & 5.7 \mathrm{E}-04 & 5.7 \mathrm{E}-04 & 2.5 \mathrm{E}-03 & 4.8 \mathrm{E}-05 & 5.9 \mathrm{E}-04 & 5.7 \mathrm{E}-04 \\ \text { ChiTd } & 8.8 \mathrm{E}-03 & 8.8 \mathrm{E}-03 & 2.2 \mathrm{E}-02 & 5.0 \mathrm{E}-04 & 9.0 \mathrm{E}-03 & 8.8 \mathrm{E}-03 \\ \text { Teen } & 5.6 \mathrm{E}-03 & 5.6 \mathrm{E}-03 & 1.0 \mathrm{E}-02 & 3.0 \mathrm{E}-04 & 5.7 \mathrm{E}-03 & 5.6 \mathrm{E}-03 \\ \text { Adu1t } & 3.1 \mathrm{E}-02 & 3.0 \mathrm{E}-02 & 4.7 \mathrm{E}-02 & 1.6 \mathrm{E}-03 & 3.1 \mathrm{E}-02 & 3.1 \mathrm{E}-02 \\ \text { T0TAL } & 4.6 \mathrm{E}-02 & 4.5 \mathrm{E}-02 & 8.1 \mathrm{E}-02 & 2.5 \mathrm{E}-03 & 4.6 \mathrm{E}-02 & 4.6 \mathrm{E}-02\end{array}$

Production/Consumption factors:

Produce: <1 Milk: $1.4 \quad$ Meat: <1

Fraction of Population Receiving an Average Individual

Total-Body Dose Commitment from Airborne Pathways

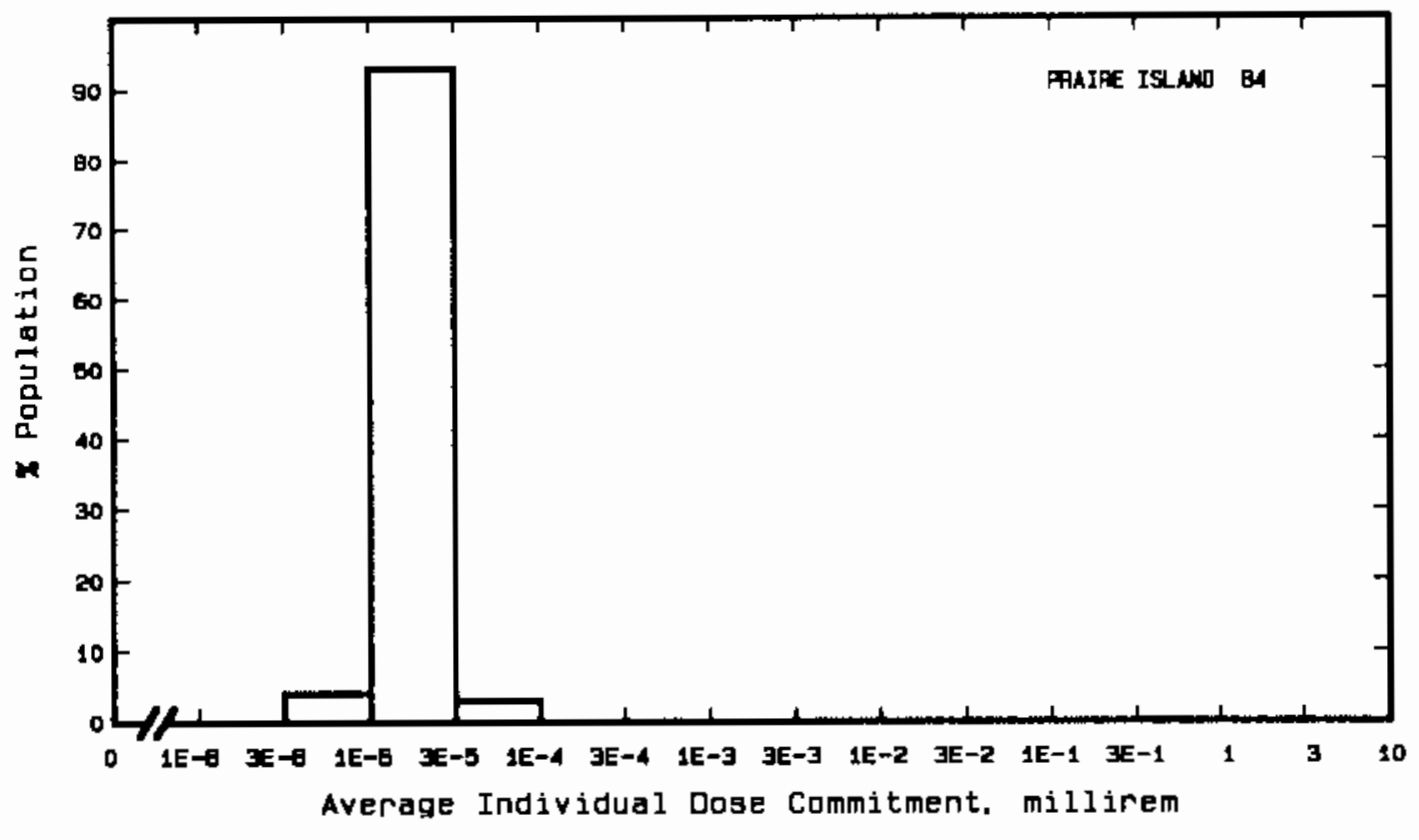


Site: QUAD CITIES

ROCK ISLAND, ILLINOIS

Location: $\quad$ N $41.7261^{\circ} \quad$ W $90.3100^{\circ}$

POPULATION DATA

Total Population Within 2-to-80-km Region: 7.2E5

Major Metropolitan Centers Within Region:

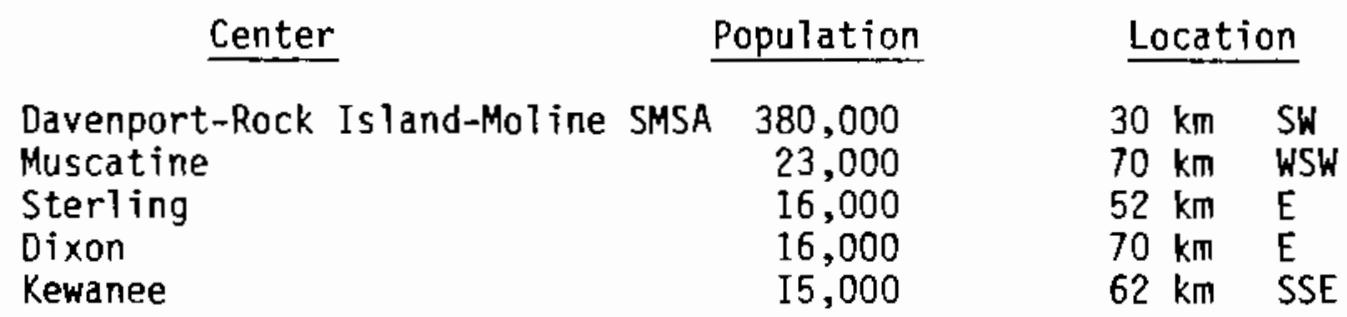

SITE-SPECIFIC DATA - AIRBORNE PATHWAYS

Average Annual State Production

Of Crops and Animal Products

In 80-km Radius Circle

Regional Productivity Factor:

Animal Grazing Factor:
Veg: 1.1E8 kilogram

MiTk: $1.8 \mathrm{E} 8$ Titer

Meat: $1.9 E 8$ kilogram

1

0.5

Meteorology Period of Record: 1 JAN $74-31$ DEC 75 Recovery: 88\%

SITE-SPECIFIC DATA - WATERBORNE PATHWAYS via MISSISSIPPI RIVER

Average River Flow

at Site: $47,000 \mathrm{ft}^{3} / \mathrm{s}$

Orinking Water:

Exposed Population: $380,000^{(a)}$

Dilution Factor: 1

Fish:

Edible Harvest: $2.1 \mathrm{E} \boldsymbol{(}^{(\mathrm{b})} \mathrm{kg} / \mathrm{yr}$
Dilution Factor: $0.5^{(\mathrm{b})}$

(a) All people in Davenport SMSA assumed to drink river water.

(b) Assumes $1 / 2$ fish harvest caught below plart. 
POPULATION DOSE-COMMITMENT ESTIMATES AND

AVERAGE INDIVIDUAL DOSE-COMMITMENT HISTOGRAM FOR

QUAD CITIES I AND 2

Dose Commitments (person-rem) from Waterborne Pathways

Total Body GI-LII Thyroid Bone Liver

$\begin{array}{llllll}\text { Infant } & 8.7 \mathrm{E}-05 & 6.8 \mathrm{E}-05 & 8.4 \mathrm{E}-04 & 1.8 \mathrm{E}-04 & 1.7 \mathrm{E}-04 \\ \text { Child } & 2.5 \mathrm{E}-03 & 1.9 \mathrm{E}-03 & 6.2 \mathrm{E}-03 & 1.0 \mathrm{E}-02 & 9.8 \mathrm{E}-02 \\ \text { Teen } & 3.0 \mathrm{E}-03 & 2.6 \mathrm{E}-03 & 2.0 \mathrm{E}-03 & 5.6 \mathrm{E}-03 & 7.4 \mathrm{E}-02 \\ \text { Adult } & 3.2 \mathrm{E}-02 & 2.3 \mathrm{E}-02 & 1.4 \mathrm{E}-02 & 3.3 \mathrm{E}-02 & 4.5 \mathrm{E}-02 \\ \text { TOTAL } & 3.7 \mathrm{E}-02 & 2.7 \mathrm{E}-02 & 2.4 \mathrm{E}-02 & 4.9 \mathrm{E}-02 & 6.2 \mathrm{E}-02\end{array}$

Dose Commitments (person-rem) from Airborne Pathways

\begin{tabular}{|c|c|c|c|c|c|c|}
\hline & Total & GI-I & Thyroid & Bone & Liver & Lung \\
\hline $\begin{array}{l}\text { fant } \\
\text { i Id } \\
\text { en }\end{array}$ & $\begin{array}{l}7.5 \mathrm{E}-03 \\
8.6 \mathrm{E}-02 \\
6.2 \mathrm{E}-02 \\
3.7 \mathrm{E}-01\end{array}$ & $\begin{array}{l}7.5 \mathrm{E}-03 \\
8.5 \mathrm{E}-02 \\
6.2 \mathrm{E}-02 \\
3.8 \mathrm{E}-01\end{array}$ & $\begin{array}{l}2.4 \mathrm{E}- \\
2.5 \mathrm{E}- \\
1.3 \mathrm{E}- \\
6.1 \mathrm{E}+\end{array}$ & $\begin{array}{l}7.7 \\
9.4 \\
6.5 \\
3.8\end{array}$ & $\begin{array}{l}7.7 \mathrm{E}-03 \\
8.6 \mathrm{E}-02 \\
6.2 \mathrm{E}-02 \\
3.7 \mathrm{E}-01\end{array}$ & $\begin{array}{l}7.8 \\
8.8 \\
6.8 \\
3.5\end{array}$ \\
\hline TAI & $5.3 \mathrm{E}-01$ & $5.3 \mathrm{E}-01$ & $1.0 E+00$ & $5.5 \mathrm{E}-01$ & $5.3 \mathrm{E}-01$ & $5.5 \mathrm{E}$ \\
\hline
\end{tabular}

Production/Consumption factors:
Produce: $<1$
Milk: 1.9
Meat: 3.2

Fraction of Population Receiving an Average Individual

Total-Body Dose Commitment from Airborne Pathways

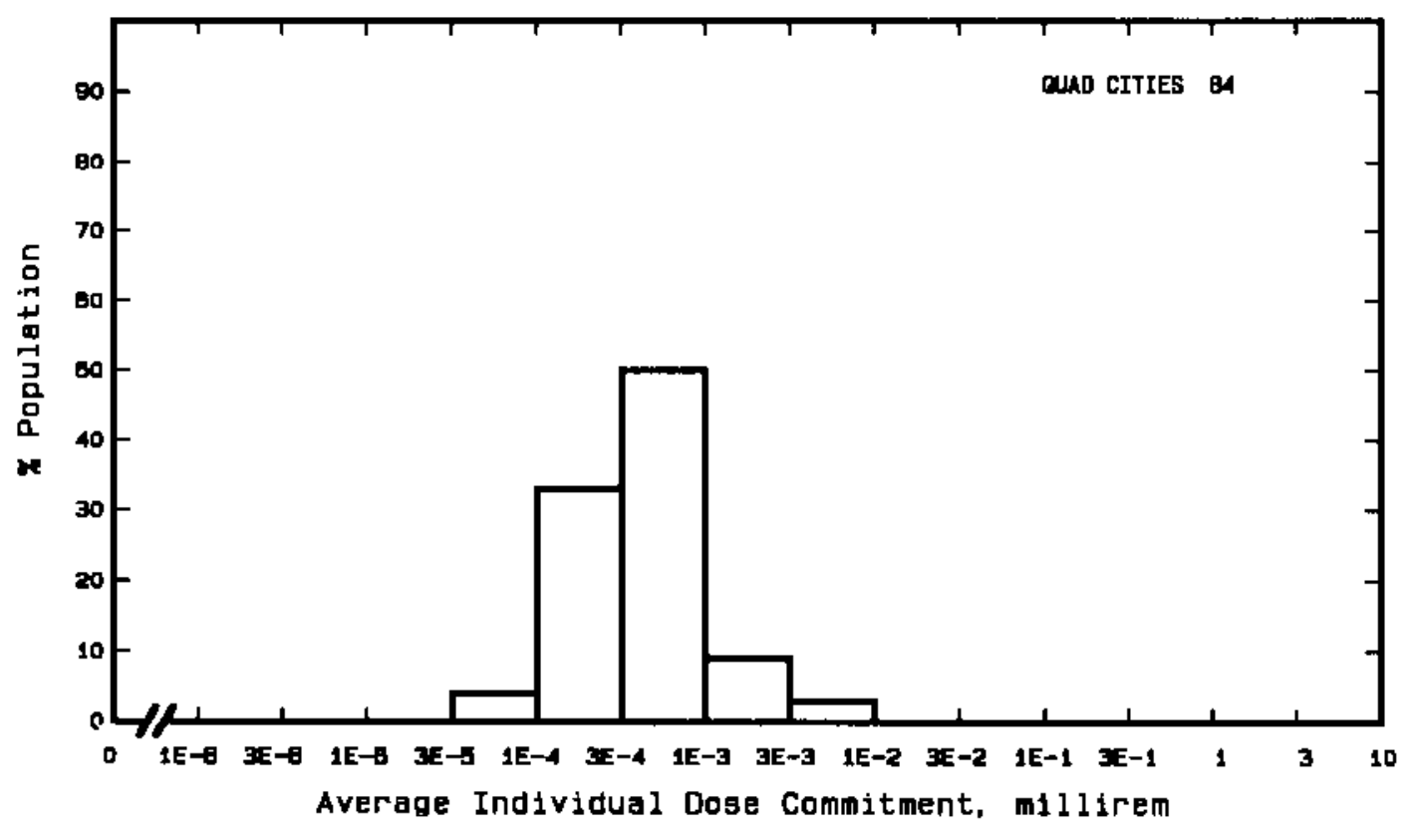


Site: RANCHO SECO

SACRAMENTO COUNTY, CALIFORNIA

Location: $\quad \mathrm{N} 38.3444^{\circ} \quad$ W $121.1200^{\circ}$

POPULATION DATA

Total Population Within 2-to-80-km Region: 1.9E6

Major Metropolitan Centers Within Region:

\begin{tabular}{lrrrr}
\multicolumn{1}{c}{ Center } & Population & \multicolumn{2}{c}{ Location } \\
\cline { 2 - 6 } Sacramento SMSA & $1,100,000$ & $42 \mathrm{~km}$ & $\mathrm{NW}$ \\
Stockton SMSA & 370,000 & $45 \mathrm{~km}$ & SSW \\
Modesto SMSA & 220,000 & $79 \mathrm{~km}$ & S \\
Antioch & 46,000 & $71 \mathrm{~km}$ & WSW \\
Davis & 39,000 & $58 \mathrm{~km}$ & WNW
\end{tabular}

SITE-SPECIFIC DATA - AIRBORNE PATHWAYS

Average Annual State Production

of Crops and Animal Products

In 80-km Radius Circle

Regionat Productivity Factor:

Animal Grazing Factor:

Meteorology Period of Record: I FEB 75 - 31 JAN 76 Recovery: 98\%
Veg: $4.8 E 7$ kilogram

Milk: 2.3E8 liter

Meat: $5.0 \mathrm{E} 7 \mathrm{ki}$ logram

1

0.9

SITE SPECIFIC DATA - WATERBORNE PATHWAYS via COSUMNES \& MOKELUMNE RIVERS

Average DiTution Flow

from Plant: $6.0 \mathrm{ft}^{3} / \mathrm{s}$

Drinking Water: Exposed Population: None

Fish: $\quad$ Edible Harvest: (a)

Dilution Factor: 0.005

(a) One percent of population is assumed to obtain fish from river. Average individual consumption rate of $7.3 \mathrm{~kg} / \mathrm{yr}$ given in FES (1973) used in lieu of catch data. 
POPULATION DOSE-COMMITMENT ESTIMATES AND

AVERAGE INDIVIDUAL DOSE-COMMITMENT HISTOGRAM FOR

RANCHO SECO

Dose Commitments (person-rem) from Waterborne Pathways

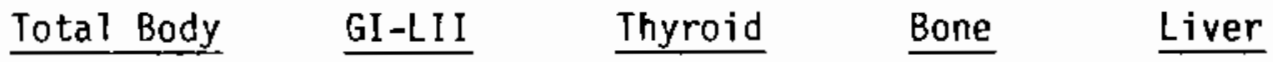

$\begin{array}{llllll}\text { Infant } & 0.0 \mathrm{E}+00 & 0.0 \mathrm{E}+00 & 0.0 \mathrm{E}+00 & 0.0 \mathrm{E}+00 & 0.0 \mathrm{E}+00 \\ \text { Chi1d } & 4.0 \mathrm{E}-01 & 1.5 \mathrm{E}-02 & 4.4 \mathrm{E}-02 & 2.0 \mathrm{E}+00 & 2.3 \mathrm{E}+00 \\ \text { Teen } & 7.7 \mathrm{E}-01 & 2.8 \mathrm{E}-02 & 3.2 \mathrm{E}-02 & 1.2 \mathrm{E}+00 & 1.9 \mathrm{E}+00 \\ \text { Adu1t } & 8.3 \mathrm{E}+00 & 2.3 \mathrm{E}-01 & 2.1 \mathrm{E}-01 & 7.0 \mathrm{E}+00 & 1.2 \mathrm{E}+01 \\ \text { T0TAL } & 9.5 \mathrm{E}+00 & 2.7 \mathrm{E}-01 & 2.8 \mathrm{E}-01 & 1.0 \mathrm{E}+01 & 1.6 \mathrm{E}+01\end{array}$

Dose Commitments (person-rem) from Airborne Pathways

Total Body GI-LII Thyroid Bone Liver

$\begin{array}{lllllll}\text { Infant } & 3.3 \mathrm{E}-03 & 3.3 \mathrm{E}-03 & 3.5 \mathrm{E}-02 & 2.0 \mathrm{E}-03 & 3.4 \mathrm{E}-03 & 3.4 \mathrm{E}-03 \\ \text { ChiTd } & 4.0 \mathrm{E}-02 & 4.0 \mathrm{E}-02 & 2.1 \mathrm{E}-01 & 2.2 \mathrm{E}-02 & 4.0 \mathrm{E}-02 & 4.2 \mathrm{E}-02 \\ \text { Teen } & 2.7 \mathrm{E}-02 & 2.7 \mathrm{E}-02 & 9.2 \mathrm{E}-02 & 1.6 \mathrm{E}-02 & 2.8 \mathrm{E}-02 & 3.0 \mathrm{E}-02 \\ \text { Adult } & 1.6 \mathrm{E}-0 \mathrm{I} & 1.6 \mathrm{E}-01 & 3.6 \mathrm{E}-01 & 9.6 \mathrm{E}-02 & 1.6 \mathrm{E}-01 & 1.7 \mathrm{E}-01 \\ \text { TOTAL } & 2.3 \mathrm{E}-01 & 2.3 \mathrm{E}-01 & 7.0 \mathrm{E}-01 & 1.4 \mathrm{E}-01 & 2.3 \mathrm{E}-01 & 2.4 \mathrm{E}-01\end{array}$

Production/Consumption factors:

Produce: <1 Milk: $1.0 \quad$ Meat: <1

Fraction of Population Receiving an Average Individual

Total-Body Bose Commitment from Airborne Pathways

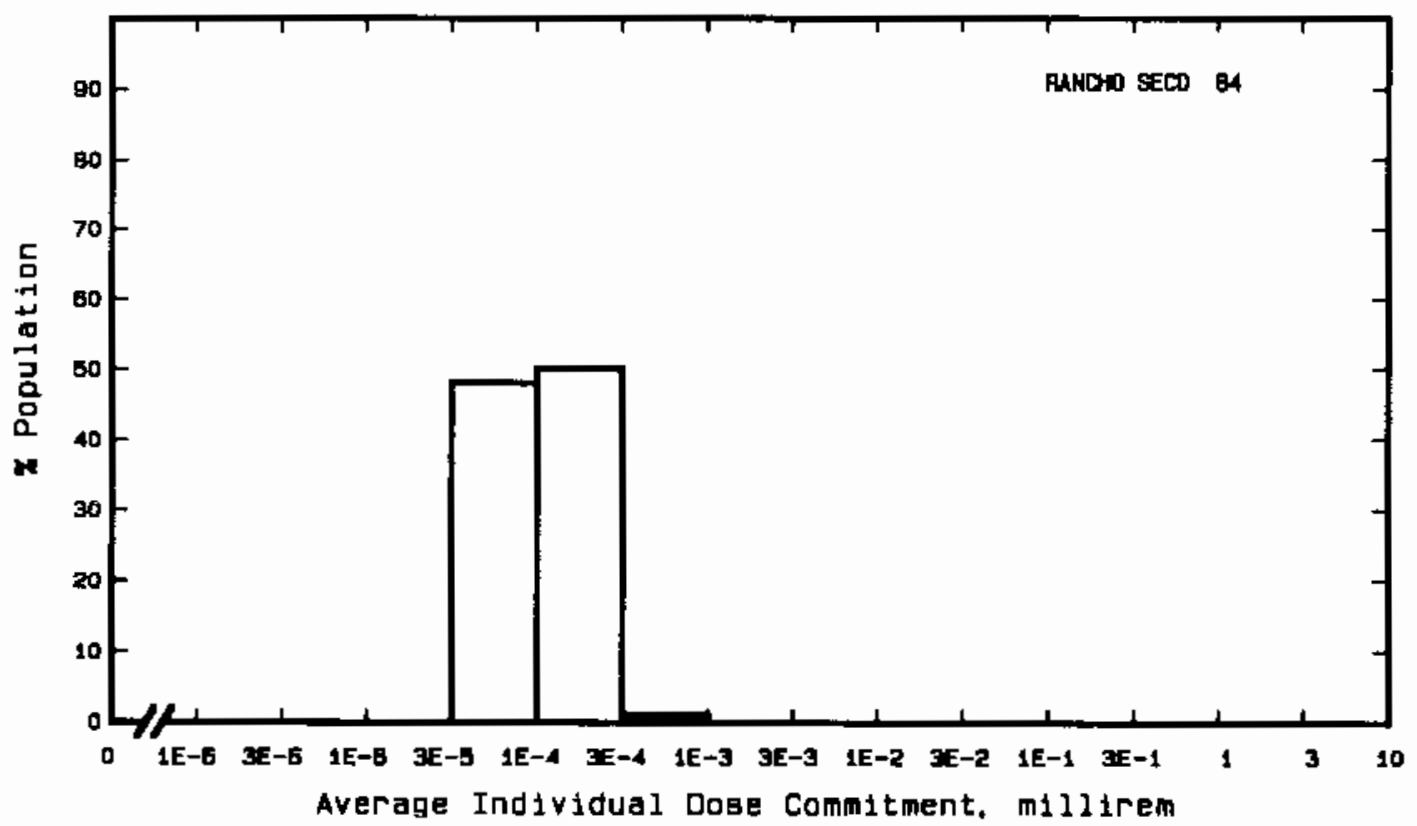


Site: H. B. ROBINSON

HARTSVILLE, SOUTH CAROLINA

Location: $\quad$ N $34.4858^{\circ}$ W $80.1586^{\circ}$

POPULATION DATA

Total Population Within 2-to-80-km Region: 6.9 E5

Major Metropolitan Centers Within Region:

Center

Florence

Sumter

Monroe

Lancaster
Population

31,000

26,000

14,000

10,000
Location

$42 \mathrm{~km} \quad \mathrm{ESE}$

$56 \mathrm{~km} \quad \mathrm{SSW}$

$74 \mathrm{~km} \quad \mathrm{NNW}$

$66 \mathrm{~km} \quad$ WNW

SITE-SPECIFIC DATA - AIRBORNE PATHWAYS

Average Annual State Production

of Crops and Animal Products

In $80-\mathrm{km}$ Radius Circle

Regional Productivity Factor:

Animal Grazing Factor:

Meteorology Period of Record: I JAN 75 - 31 DEC 75 Recovery: 94\%
Veg: 7.4E6 kilogram

Milk: 5.7 E7 liter

Meat: $5.0 \mathrm{E} 7$ kilogram

1

0.8

SITE-SPECIFIC DATA - WATERBORNE PATHWAYS via LAKE ROBINSON

Drinking Water:

Fish:
Average Dilution Flow

from Plant: $480 \mathrm{ft}^{3} / \mathrm{s}$

Exposed Pofulation: None

Edible Harvest: $1.8^{(\mathrm{a})} \mathrm{kg} / \mathrm{yr}$

(a) Average individual consumption rate as given in the FES (1975) used in Tieu of catch data.

(b) Ten percent of population consumes fish taken from water diluted by a factor of 0.2 according to FES. 
POPULATION DOSE-COMMITMENT ESTIMATES AND

AVERAGE INDIVIDUAL DOSE-COMMITMENT HISTOGRAM FOR

\author{
H. B. ROBINSON
}

Dose Commitments (person-rem) from Waterborne Pathways

\begin{tabular}{|c|c|c|c|c|c|}
\hline & Tota 1 Body & $\underline{G I-L I I}$ & Thyroid & Bone & Liver \\
\hline $\begin{array}{l}\text { Infant } \\
\text { Child } \\
\text { Teen } \\
\text { Adult }\end{array}$ & $\begin{array}{l}0.0 \mathrm{E}+00 \\
1.1 \mathrm{E}-02 \\
2.1 \mathrm{E}-02 \\
2.3 \mathrm{E}-01\end{array}$ & $\begin{array}{l}0.0 \mathrm{E}+00 \\
1.2 \mathrm{E}-03 \\
2.4 \mathrm{E}-03 \\
2.1 \mathrm{E}-03\end{array}$ & $\begin{array}{l}0.0 \mathrm{E}+00 \\
1.5 \mathrm{E}-03 \\
1.1 \mathrm{E}-03 \\
7.3 \mathrm{E}-03\end{array}$ & $\begin{array}{l}0.0 \mathrm{E}+00 \\
5.0 \mathrm{E}-02 \\
3.0 \mathrm{E}-02 \\
1.8 \mathrm{E}-01\end{array}$ & $\begin{array}{l}0.0 \mathrm{E}+00 \\
6.0 \mathrm{E}-02 \\
5.1 \mathrm{E}-02 \\
3.0 \mathrm{E}-01\end{array}$ \\
\hline OTAL & $2.6 \mathrm{E}-01$ & 2.4E-02 & $9.9 \mathrm{E}-03$ & $2.6 \mathrm{E}-01$ & $4.2 E-01$ \\
\hline
\end{tabular}

Dose Commitments (person-rem) from Airborne Pathways

Total Body GI-LII Thyroid Bone Liver $\underline{\text { Lung }}$

$\begin{array}{lllllll}\text { Infant } & 1.7 \mathrm{E}-05 & 1.7 \mathrm{E}-05 & 1.1 \mathrm{E}-04 & 1.1 \mathrm{E}-05 & 1.7 \mathrm{E}-05 & 1.8 \mathrm{E}-05 \\ \text { Child } & 2.1 \mathrm{E}-04 & 2.1 \mathrm{E}-04 & 7.0 \mathrm{E}-04 & 1.2 \mathrm{E}-04 & 2.1 \mathrm{E}-04 & 2.3 \mathrm{E}-04 \\ \text { Teen } & 1.5 \mathrm{E}-04 & 1.5 \mathrm{E}-04 & 3.5 \mathrm{E}-04 & 8.7 \mathrm{E}-05 & 1.5 \mathrm{E}-04 & 1.7 \mathrm{E}-04 \\ \text { Adult } & 9.0 \mathrm{E}-04 & 9.0 \mathrm{E}-04 & 1.6 \mathrm{E}-03 & 5.3 \mathrm{E}-04 & 9.0 \mathrm{E}-04 & 9.7 \mathrm{E}-04 \\ \text { TOTAL } & 1.3 \mathrm{E}-03 & 1.3 \mathrm{E}-03 & 2.8 \mathrm{E}-03 & 7.4 \mathrm{E}-04 & 1.3 \mathrm{E}-03 & 1.4 \mathrm{E}-03\end{array}$

Production/Consumption factors:

Produce: <1 Milk: <1 Meat: $<1$

Fraction of Population Receiving an Average Individual Total-Body Dose Commitment from Airborne Pathways

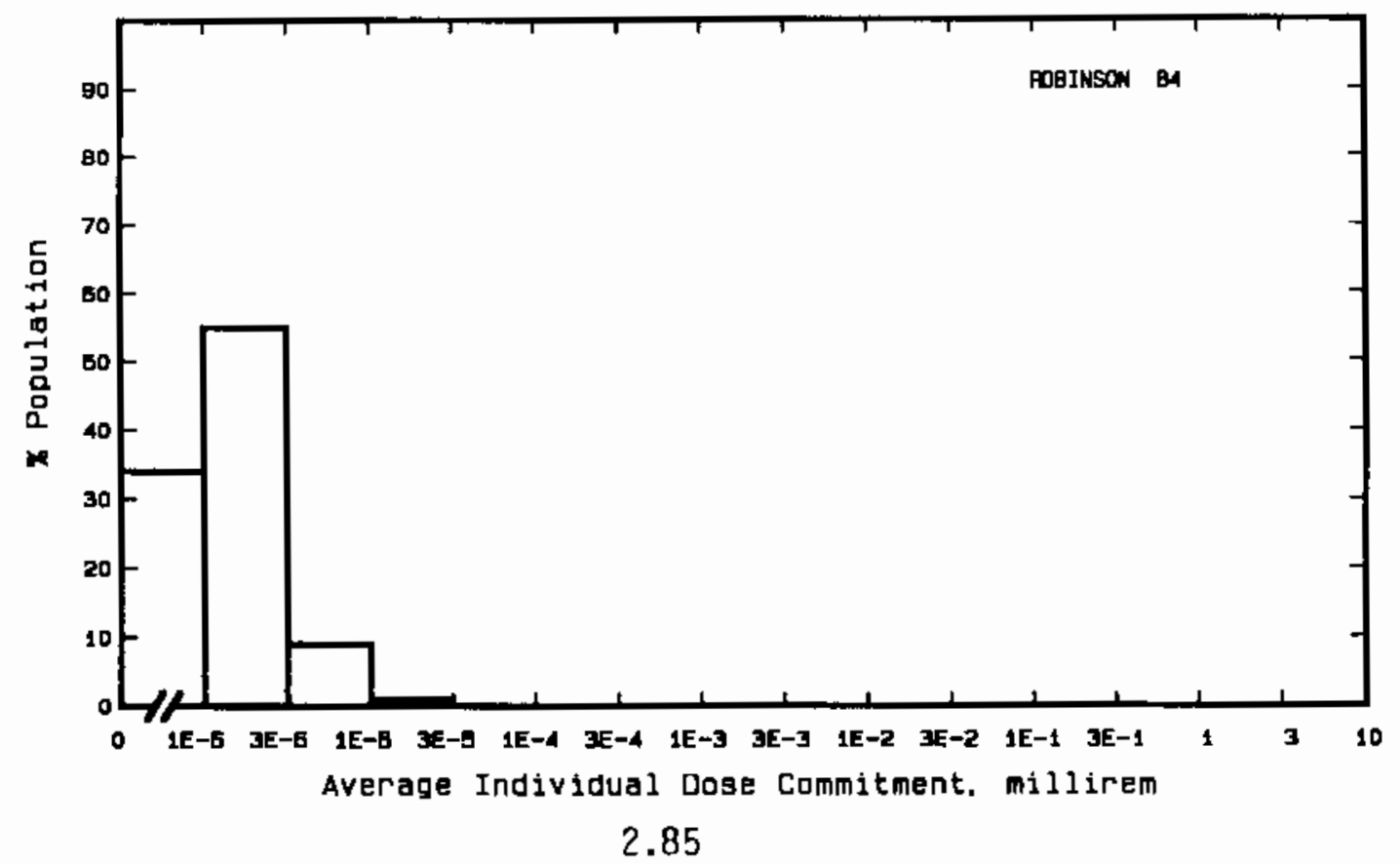


Site: ST. LUCIE

FORT PIERCE, FLORIDA

Location: $\quad \mathrm{N} 27.3486^{\circ}$ W $80.2464^{\circ}$

POPULATION DATA

Total Population Within 2-to-80-km Region: $6.3 \mathrm{E} 5$

Major Metropolitan Centers Within Region:

\begin{tabular}{|c|c|c|c|}
\hline Center & Population & \multicolumn{2}{|c|}{ Location } \\
\hline West PaIm Beach & 69,000 & $73 \mathrm{~km}$ & SSE \\
\hline Ft. Pierce & 37,000 & $14 \mathrm{~km}$ & NW \\
\hline Riviera Beach & 28,000 & $65 \mathrm{~km}$ & SSE \\
\hline Vero Beach & 18,000 & $36 \mathrm{~km}$ & NNW \\
\hline Palm Beach & 11,000 & $72 \mathrm{~km}$ & SSE \\
\hline
\end{tabular}

SITE-SPECIFIC DATA - AIRBORNE PATHWAYS

Average Annual State Production

of Crops and Animal Products

In 80-km Radius Circle

Veg: $2.8 \mathrm{E} 7 \mathrm{kilogram}$

Milk: 1.1 E8 liter

Meat: $7.2 E 7$ kilogram

Regional Productivity Factor:

Animal Grazing Factor:

0.5

1

Meteorology Period of Record: 1 JAN 76 - 31 DEC 76 Recovery: 92\%

SITE-SPECIFIC DATA - WATERBORNE PATHWAYS via ATLANTIC OCEAN

Average DiTution Flow

from Plant: $3,800 \mathrm{ft}^{3} / \mathrm{s}$

Fish:

Edible Harvest: $2.6 E 5 \mathrm{~kg} / \mathrm{yr}$
Dilution Factor: $0.005 \mathrm{~g} / \mathrm{s}$

Invertebrates:

Edible Harvest: $2.7 \mathrm{E} 4 \mathrm{~kg} / \mathrm{yr}$
Dilution Factor: 0.005

(a) Dilution factors as given in FES (1973). 
POPULATION DOSE-COMMITMENT ESTIMATES AND

AVERAGE INDIVIDUAL. DOSE-COMMITMENT HISTOGRAM FOR

SAINT LUCIE 1 AND 2

Dose Commitments (person-rem) from Waterborne Pathways

\begin{tabular}{|c|c|c|c|c|c|}
\hline & Total Body & $\underline{G I-L I I}$ & Thyroid & Bone & Liver \\
\hline $\begin{array}{l}\text { Infant } \\
\text { Child } \\
\text { Teen } \\
\text { Adult }\end{array}$ & $\begin{array}{l}0.0 \mathrm{E}+00 \\
2.6 \mathrm{E}-04 \\
2.6 \mathrm{E}-04 \\
2.2 \mathrm{E}-03\end{array}$ & $\begin{array}{l}0.0 E+00 \\
4.8 E-04 \\
9.9 E-04 \\
8.5 E-03\end{array}$ & $\begin{array}{l}0.0 \mathrm{E}+00 \\
8.8 \mathrm{E}-04 \\
6.3 \mathrm{E}-04 \\
4.1 \mathrm{E}-03\end{array}$ & $\begin{array}{l}0.0 \mathrm{E}+00 \\
7.4 \mathrm{E}-04 \\
4.3 \mathrm{E}-04 \\
2.5 \mathrm{E}-03\end{array}$ & $\begin{array}{l}0.0 \mathrm{E}+00 \\
7.3 \mathrm{E}-04 \\
6.1 \mathrm{E}-04 \\
3.6 \mathrm{E}-03\end{array}$ \\
\hline TOTAL & $2.8 \mathrm{E}-03$ & $1.0 \mathrm{E}-02$ & $5.6 \mathrm{E}-03$ & $3.7 \mathrm{E}-03$ & 4. $9 E-03$ \\
\hline
\end{tabular}

Dose Commitments (person-rem) from Airborne Pathways

\begin{tabular}{lccccccc} 
& Total Body & GI-LII & Thyroid & & Bone & Liver & Lung \\
\cline { 2 - 3 } Infant & $1.9 \mathrm{E}-02$ & $1.9 \mathrm{E}-02$ & $2.5 \mathrm{E}-01$ & $1.7 \mathrm{E}-02$ & $1.9 \mathrm{E}-02$ & $2.0 \mathrm{E}-02$ \\
Child & $2.2 \mathrm{E}-01$ & $2.2 \mathrm{E}-01$ & $1.5 \mathrm{E}-00$ & $1.8 \mathrm{E}-01$ & $2.3 \mathrm{E}-01$ & $2.4 \mathrm{E}-01$ \\
Teen & $1.6 \mathrm{E}-01$ & $1.6 \mathrm{E}-01$ & $7.1 \mathrm{E}-01$ & $1.3 \mathrm{E}-01$ & $1.6 \mathrm{E}-01$ & $1.8 \mathrm{E}-01$ \\
Adult & $9.7 \mathrm{E}-01$ & $9.7 \mathrm{E}-01$ & $2.9 \mathrm{E}-00$ & $8.0 \mathrm{E}-01$ & $9.7 \mathrm{E}-01$ & $1.0 \mathrm{E}-01$ \\
TOTAL & $1.4 \mathrm{E}-00$ & $1.4 \mathrm{E}-00$ & $5.4 \mathrm{E}+00$ & $1.1 \mathrm{E}-01$ & $1.4 \mathrm{E}-00$ & $1.5 \mathrm{E}-00$
\end{tabular}

Production/Consumption factors:

Produce: <1 Milk: <1 Meat: <1

Fraction of Population Receiving an Average Individual

Total-Body Dose Commitment from Airborne Pathways

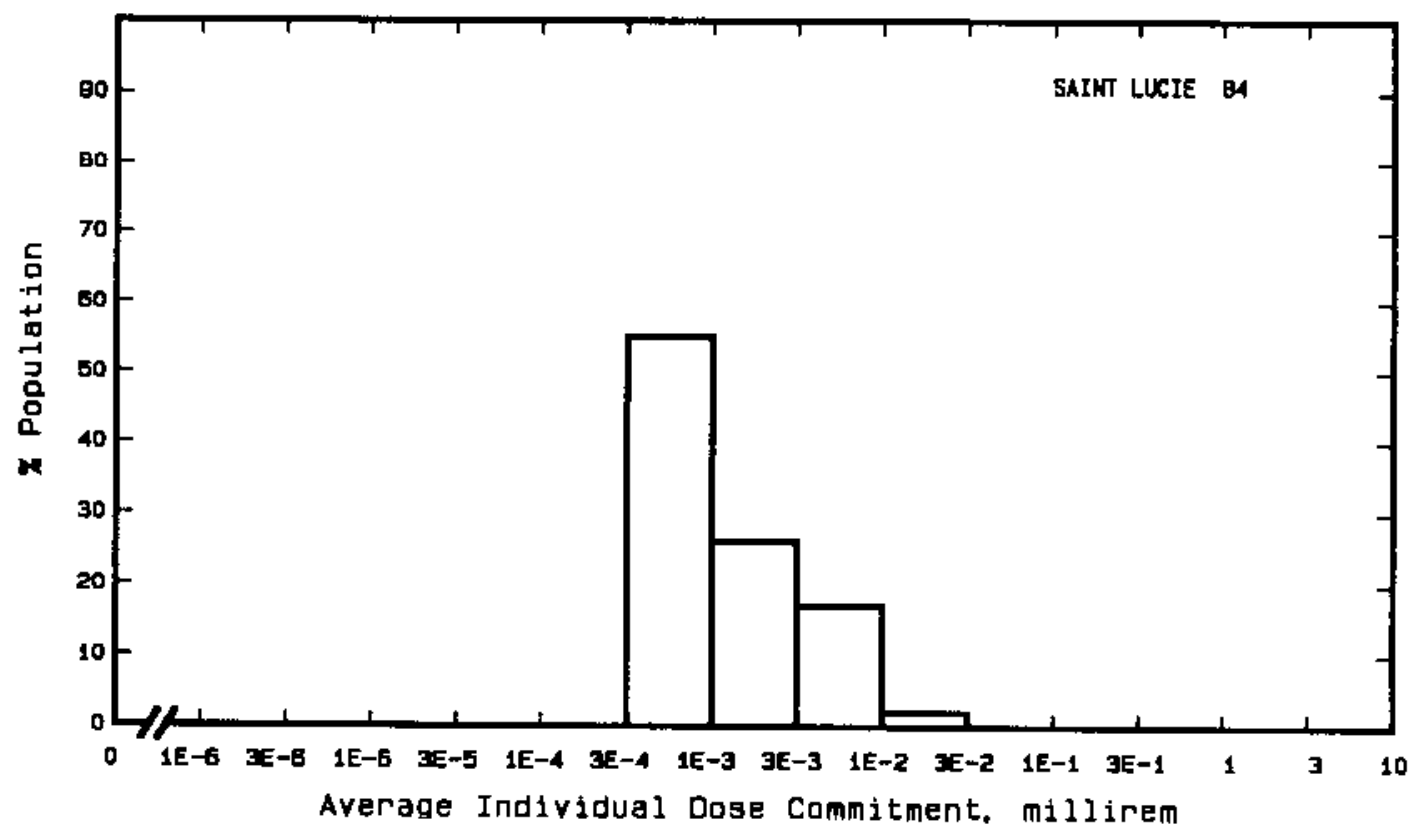


Site: SALEM

SALEN, NEW JERSEY

Location: N $39.4628^{\circ}$ W $75.5358^{\circ}$

POPULATION DATA

Total Population Within 2-to-80-km Region: 4.7E6

Major Metropolitan Centers Within Region:

Center

Philadelphia SMSA

Wilmington SMSA

VineTand-Millville-Bridgeton SMSA

Chester

Norristown

Population Location

4,700,000 $\quad 63 \mathrm{~km}$ NNE

$400,000 \quad 32 \mathrm{~km} \quad \mathrm{NNW}$

$130,000 \quad 48 \mathrm{~km} \quad E$

$46,000 \quad 45 \mathrm{~km} \quad \mathrm{NNE}$

SITE-SPECIFIC DATA - AIRBORNE PATHWAYS

Average Annual State Production

of Crops and Animal Products

In 80-km Radius Circle

Regional Productivity Factor:

Animal Grazing Factor:

Meteorology Period of Record: 1 JUN 70 - 31 MAY 71 Recovery: 95\%
Veg: $7.4 \mathrm{E} 7 \mathrm{ki}$ logram

Milk: 2.7E8 liter

Meat: $2.4 \mathrm{E} 7$ kilogram

0.9

0.6

SITE-SPECIFIC DATA - WATERBORNE PATHWAYS via DELAWARE RIVER ESTUARY

Average River Flow at Site: $16,000 \mathrm{ft}^{3} / \mathrm{s}$

Fish:

Edible Harvest: $3.6 \mathrm{E} 5 \mathrm{~kg} / \mathrm{yr}$ Dilution Factor: 1

Invertebrates: (a)

Edible Harvest: $1.6 \mathrm{E} 5 \mathrm{~kg} / \mathrm{yr}$ Dilution Factor: 1

(a) Environment primarily salt water so invertebrates considered in lieu of drinking water. 
POPULATION DOSE-COMMITMENT ESTIMATES AND

AVERAGE INDIVIDUAL DOSE-COMMITMENT HISTOGRAM FOR

SALEM 1 AND 2

Dose Commitments (person-rem) from Waterborne Pathways

\begin{tabular}{|c|c|c|c|c|c|}
\hline & Total Body & GI-LII & Thyroid & Bone & Liver \\
\hline $\begin{array}{l}\text { Infant } \\
\text { Child } \\
\text { Teen } \\
\text { Adult }\end{array}$ & $\begin{array}{l}0.0 \mathrm{E}+00 \\
4.8 \mathrm{E}-02 \\
3.4 \mathrm{E}-02 \\
2.1 \mathrm{E}-01\end{array}$ & $\begin{array}{l}0.0 \mathrm{E}+00 \\
1.1 \mathrm{E}-01 \\
2.3 \mathrm{E}-01 \\
2.0 \mathrm{E}+00\end{array}$ & $\begin{array}{l}0.0 \mathrm{E}+00 \\
1.4 \mathrm{E}-02 \\
9.8 \mathrm{E}-03 \\
6.5 \mathrm{E}-02\end{array}$ & $\begin{array}{l}0.0 \mathrm{E}+00 \\
9.0 \mathrm{E}-\mathrm{D} 3 \\
5.5 \mathrm{E}-03 \\
3.3 \mathrm{E}-02\end{array}$ & $\begin{array}{l}0.0 \mathrm{E}+00 \\
3.2 \mathrm{E}-02 \\
2.8 \mathrm{E}-02 \\
1.7 \mathrm{E}-01\end{array}$ \\
\hline TOTAL & $2.9 \mathrm{E}-01$ & $2.4 \mathrm{E}+00$ & $8.8 \mathrm{E}-02$ & 4.7E-02 & 2. $3 \mathrm{~F}-01$ \\
\hline
\end{tabular}

Dose Commitments (person-rem) from Airborne Pathways Total Body GI-LII Thyroid Bone Liver Lung

$\begin{array}{lllllll}\text { Infant } & 2.9 \mathrm{E}-03 & 2.8 \mathrm{E}-03 & 5.1 \mathrm{E}-03 & 4.0 \mathrm{E}-04 & 2.9 \mathrm{E}-03 & 2.9 \mathrm{E}-03\end{array}$

$\begin{array}{lllllll}\text { Child } & 4.1 \mathrm{E}-02 & 4.1 \mathrm{E}-02 & 5.4 \mathrm{E}-02 & 4.2 \mathrm{E}-03 & 4.2 \mathrm{E}-02 & 4.2 \mathrm{E}-02\end{array}$

TOTAL 2.3E-01 2.3E-01 $2.7 \mathrm{E}-01 \quad 2.4 \mathrm{E}-02 \quad 2.3 \mathrm{E}-01 \quad 2.3 \mathrm{E}-01$

Production/Consumption factors:

Produce: <1 Milk: <1 Meat: <1

Fraction of Population Receiving an Average Individual

Total-Body Dose Commitment from Airborne Pathways

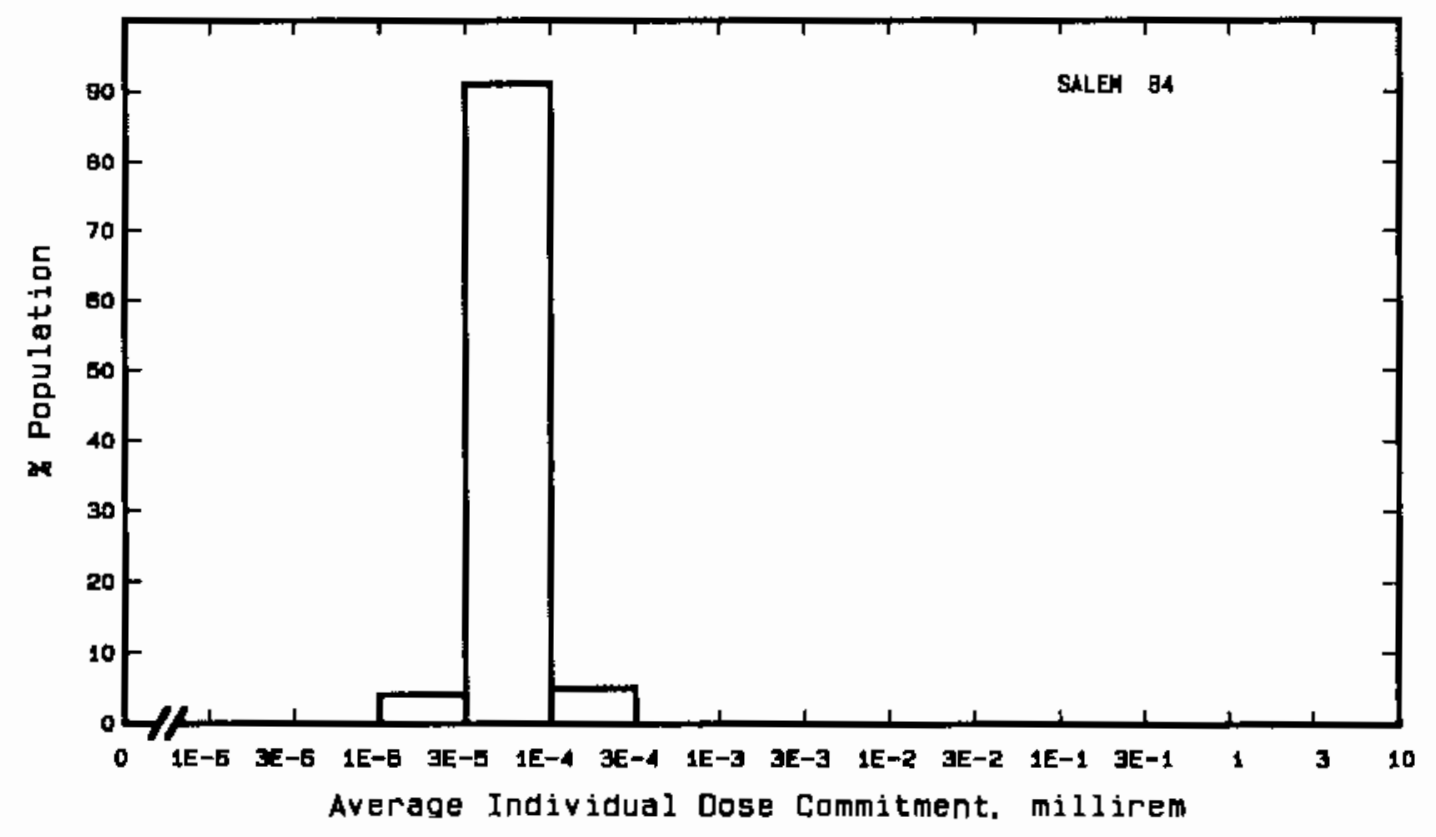


Site: SAN ONOFRE

Location: $\quad$ N $33.3703^{\circ}$ W $117.5569^{\circ}$

POPULATION DATA

Total Population Within 2-to-80-km Region: $5.0 E 6$

Major Metropolitan Centers Within Region:

Center Population Location

San Diego SMSA $2,000,000$

Anaheim-Santa Ana-Garden Grove SMSA 2,000,000

$68 \mathrm{~km} \quad$ SSE

Long Beach

Huntington 8each

Riverside

Pomona

380,000

180,000

180,000

100,000

$62 \mathrm{~km} \quad \mathrm{NW}$

$75 \mathrm{~km} \quad \mathrm{NW}$

$61 \mathrm{~km} \quad \mathrm{NW}$

$68 \mathrm{~km} \quad \mathrm{~N}$

$79 \mathrm{~km} \quad \mathrm{NNW}$

\section{SITE-SPECIFIC DATA - AIRBORNE PATHWAYS}

Average Annual State Production

of Crops and Animal Products

In 80-km Radius Circle

Regional Productivity Factor:

Animal Grazing Factor:

Meteorology Period of Record: 25 JAN 73 - 24 JAN 76 Recovery: $88 \%$
Veg: $4.8 E 7$ kilogram

Milk: 2.3E8 liter

Meat: $5.0 \mathrm{E} 7$ kilogram

0.6

1

SITE-SPECIFIC DATA - WATERBORNE PATHWAYS via PACIFIC OCEAN

$\begin{array}{ll}\text { Fish: } & \text { Edible Harvest: } 2.9 \mathrm{E}^{(\mathrm{a})} \\ & \text { Dilution Factor: } 1 \\ & \text { Edible Harvest: } 2.9 \mathrm{E}^{(\mathrm{a})} \mathrm{kg} / \mathrm{yr} \\ & \text { Dilution Factor: } 1\end{array}$

Average Dilution Flow

from Plant: $470 \mathrm{ft}^{3} / \mathrm{s}$

(a) Seafood caught in undiluted effluent according to FES (1973). 
POPULATION DOSE-COMMITMENT ESTIMATES AND

AVERAGE INDIVIDUAL DOSE-COMMITMENT HISTOGRAM FOR

SAN ONOFRE 1,2 , AND 3

Dose Commitments (person-rem) from Waterborne Pathways

Tota? Body GI-LII Thyroid Bone Liver

$\begin{array}{llllll}\text { Infant } & 0.0 \mathrm{E}+00 & 0.0 \mathrm{E}+00 & 0.0 \mathrm{E}+00 & 0.0 \mathrm{E}+00 & 0.0 \mathrm{E}+00 \\ \text { Child } & 1.8 \mathrm{E}-01 & 1.3 \mathrm{E}+00 & 2.1 \mathrm{E}-01 & 3.5 \mathrm{E}-01 & 4.3 \mathrm{E}-01 \\ \text { Teen } & 1.6 \mathrm{E}-01 & 2.6 \mathrm{E}+00 & 1.5 \mathrm{E}-01 & 2.1 \mathrm{E}-01 & 3.6 \mathrm{E}-01 \\ \text { Adult } & 1.3 \mathrm{E}+00 & 2.3 \mathrm{E}+01 & 1.0 \mathrm{E}+00 & 1.2 \mathrm{E}+00 & 2.2 \mathrm{E}-00 \\ \text { TOTAL } & 1.7 \mathrm{E}+00 & 2.7 \mathrm{E}+01 & 1.4 \mathrm{E}+00 & 1.8 \mathrm{E}+00 & 2.9 \mathrm{E}-00\end{array}$

Dose Commitments (person-rem) from Airborne Pathways

Total Body GI-LII Thyroid Bone Liver Lung

$\begin{array}{lllllll}\text { Infant } & 3.0 \mathrm{E}-02 & 2.9 \mathrm{E}-02 & 5.3 \mathrm{E}-01 & 2.8 \mathrm{E}-02 & 3.1 \mathrm{E}-02 & 3.1 \mathrm{E}-02 \\ \text { Child } & 3.4 \mathrm{E}-01 & 3.4 \mathrm{E}-01 & 3.1 \mathrm{E}+00 & 3.0 \mathrm{E}-01 & 3.4 \mathrm{E}-01 & 3.7 \mathrm{E}-01 \\ \text { Teen } & 2.5 \mathrm{E}-01 & 2.5 \mathrm{E}-01 & 1.3 \mathrm{E}+00 & 2.2 \mathrm{E}-01 & 2.5 \mathrm{E}-01 & 2.9 \mathrm{E}-01 \\ \text { Aduit } & 1.5 \mathrm{E}+00 & 1.5 \mathrm{E}+00 & 5.1 \mathrm{E}+00 & 1.3 \mathrm{E}+00 & 1.5 \mathrm{E}+00 & 1.6 \mathrm{E}+00 \\ \text { TOTAL } & 2.1 \mathrm{E}+00 & 2.1 \mathrm{E}+00 & 1.0 \mathrm{E}+01 & 1.9 \mathrm{E}+00 & 2.1 \mathrm{E}+00 & 2.3 \mathrm{E}+00\end{array}$

Production/Consumption factors:

Produce: <l Milk: <1 Meat: <1

Fraction of Population Receiving an Average Individual

Total-Body Dose Commitment from Airborne Pathways

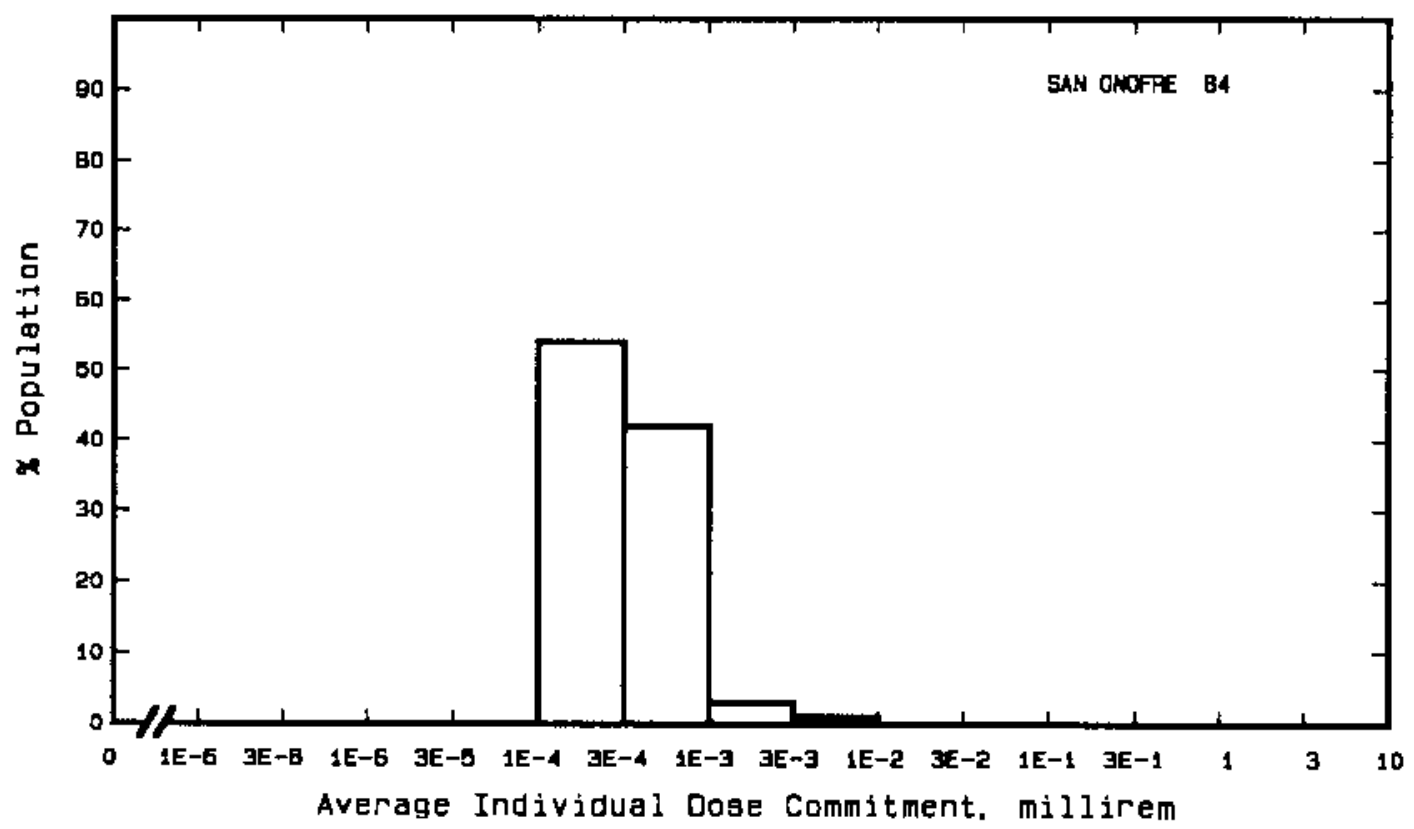


Site: SEQUOYAH

Location: $\quad \mathrm{N} 35.2233^{\circ} \quad$ W $85.0878^{\circ}$

POPULATION DATA

Total Population Within 2-to-80-km Region: 8.4E5

Major Metropolitan Centers Within Region:

Center
Chattanooga
Cleveland
East Ridge
0alton
Athens

\begin{tabular}{ccccc} 
& Population & & \multicolumn{2}{c}{ Location } \\
\cline { 2 - 4 } & & & \\
SMSA & 440,000 & & $28 \mathrm{~km}$ & SW \\
& 26,000 & & $21 \mathrm{~km}$ & SE \\
& 21,000 & $27 \mathrm{~km}$ & SSW \\
& 21,000 & $50 \mathrm{~km}$ & $\mathrm{~S}$ \\
& 12,000 & $53 \mathrm{~km}$ & ENE
\end{tabular}

SITE-SPECIFIC DATA - AIRBORNE PATHWAYS

Average Annual State Production

of Crops and Animal Products

In 80-km Radius Circle

Regional Productivity Factor:

Animal Grazing Factor:

Meteorology Period of Record: I JAN 72 - 3I DEC 75
Veg: $1.1 E 7$ kilogram

Milk: $1.6 \mathrm{E} 8$ liter

Meat: $1.2 \mathrm{E} 8 \mathrm{ki}$ logram

0.25

0.7

Recovery: $93 \%$

SITE-SPECIFIC DATA - WATERBORNE PATHWAYS via TENNESSEE RIVER

Average River Flow

at Site: $35,000 \mathrm{ft}^{3} / \mathrm{s}$

Drinking Water:

Exposed Population: 430,000

Dilution Factor: 1

Fish:

Edible Harvest: $1.7 \mathrm{ES}^{(\mathrm{a})} \mathrm{kg} / \mathrm{yr}$

Dilution Factor: i

(a) Catch data given in FES (1974). 
POPULATION DOSE-COMMITMENT ESTIMATES AND

AVERAGE INDIVIDUAL DOSE-COMMITMENT HISTOGRAM FOR

SEQUOYAH I AND 2

Dose Commitments (person-rem) from Waterborne Pathways

Total Body GI-LII Thyroid Bone Liver

$\begin{array}{llllll}\text { Infant } & 2.1 \mathrm{E}-02 & 2.0 \mathrm{E}-02 & 6.1 \mathrm{E}-02 & 4.5 \mathrm{E}-03 & 2.6 \mathrm{E}-02 \\ \text { Child } & 2.5 \mathrm{E}-01 & 2.4 \mathrm{E}-01 & 5.1 \mathrm{E}-01 & 9.5 \mathrm{E}-02 & 3.3 \mathrm{E}-01 \\ \text { Teen } & 1.1 \mathrm{E}-01 & 1.1 \mathrm{E}-01 & 1.7 \mathrm{E}-01 & 4.0 \mathrm{E}-02 & 1.5 \mathrm{E}-01 \\ \text { Adult } & 1.0 \mathrm{E}+00 & 9.5 \mathrm{E}-01 & 1.3 \mathrm{E}+00 & 2.4 \mathrm{E}-01 & 1.1 \mathrm{E}-00 \\ \text { TOTAL } & 1.4 \mathrm{E}+00 & 1.3 \mathrm{E}+00 & 2.1 \mathrm{E}+00 & 3.8 \mathrm{E}-01 & 1.6 \mathrm{E}+00\end{array}$

Dose Comitments (person-rem) from Airborne Pathways

Total Body GI-LII Thyroid $\underline{\text { 8one Liver Lung }}$

$\begin{array}{lllllll}\text { Infant } & 5.2 \mathrm{E}-03 & 5.2 \mathrm{E}-03 & 6.4 \mathrm{E}-03 & 4.1 \mathrm{E}-03 & 5.2 \mathrm{E}-03 & 5.6 \mathrm{E}-03 \\ \text { Child } & 6.5 \mathrm{E}-02 & 6.5 \mathrm{E}-02 & 7.4 \mathrm{E}-02 & 4.6 \mathrm{E}-02 & 6.5 \mathrm{E}-02 & 7.0 \mathrm{E}-02 \\ \text { Teen } & 4.8 \mathrm{E}-02 & 4.8 \mathrm{E}-02 & 5.3 \mathrm{E}-02 & 3.3 \mathrm{E}-02 & 4.8 \mathrm{E}-02 & 5.5 \mathrm{E}-02 \\ \text { Adult } & 2.9 \mathrm{E}-01 & 2.9 \mathrm{E}-01 & 3.1 \mathrm{E}-01 & 2.0 \mathrm{E}-01 & 2.9 \mathrm{E}-01 & 3.1 \mathrm{E}-01 \\ \text { TOTAL } & 4.1 \mathrm{E}-01 & 4.1 \mathrm{E}-01 & 4.4 \mathrm{E}-01 & 2.8 \mathrm{E}-01 & 4.1 \mathrm{E}-01 & 4.4 \mathrm{E}-01\end{array}$

Production/Consumption factors:

Produce: <1 Milk: <1 Meat: <1

Fraction of Population Receiving an Average Individual Total-Body Dose Commitment from Airborne Pathways

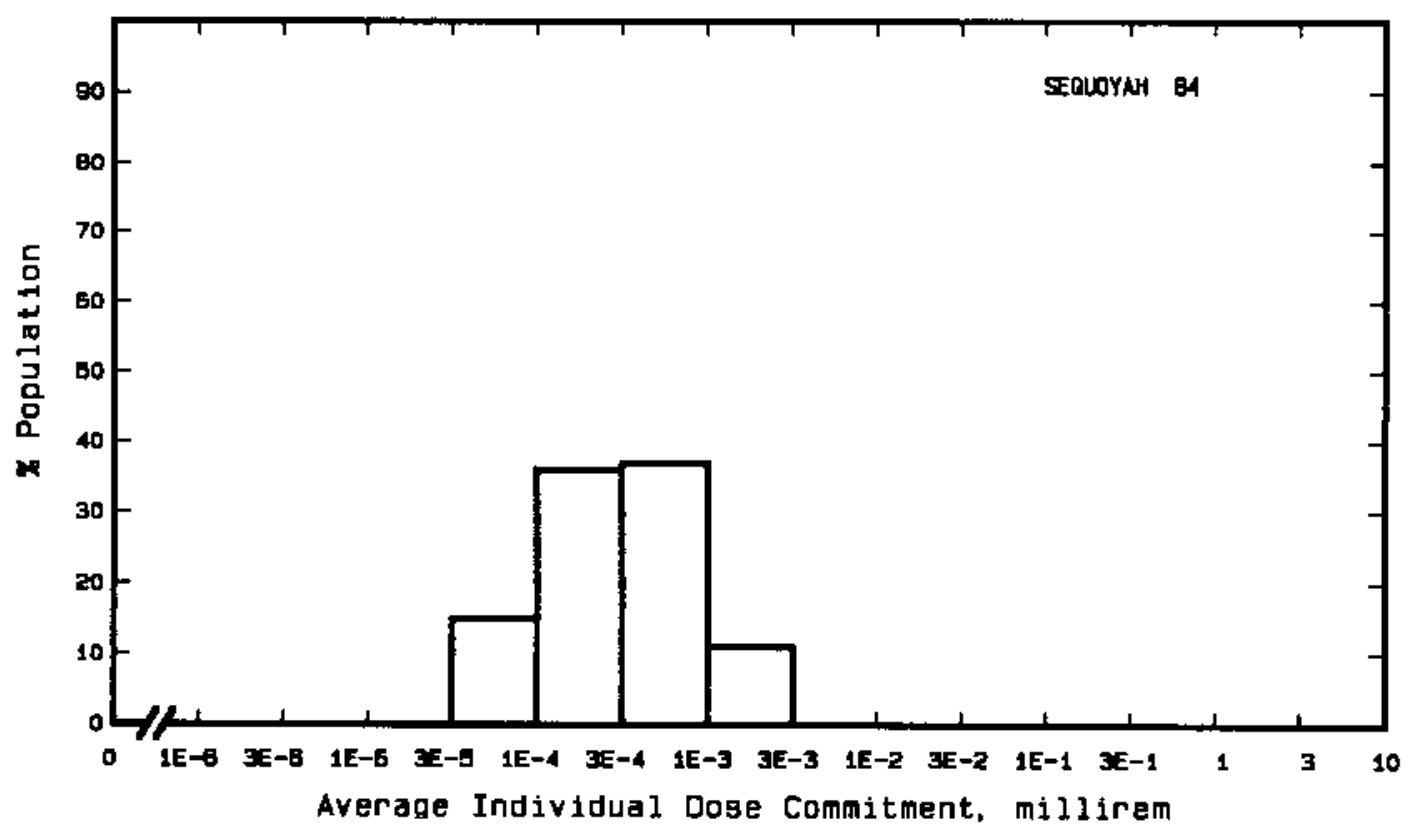


Site: SUMMER

JENKINSVILLE, SOUTH CAROLINA

Location: $\quad$ N $34.2958^{\circ} \quad$ W $81.3203^{\mathrm{C}}$

POPULATION DATA

Total Population Within 2-to-80-km Region: $8.5 \mathrm{E5}$

Major Metropolitan Centers Within Region:

\begin{tabular}{lcccc} 
Center & Population & \multicolumn{2}{c}{ Location } \\
\cline { 2 - 3 } SMSA & 430,000 & & $42 \mathrm{~km}$ & SE \\
Columb & 36,000 & $75 \mathrm{~km}$ & NNE \\
Rock Hill & 23,000 & $78 \mathrm{~km}$ & W \\
Greenwood & 11,000 & $54 \mathrm{~km}$ & NNW \\
Union & 11,000 & $68 \mathrm{~km}$ & WNW
\end{tabular}

SITE-SPECIFIC DATA - AIRBORNE PATHWAYS

Average Annua T State Production

of Crops and Animal Products

In 80-km Radius Circle

Regional Productivity Factor:

Animat Grazing Factor:

Meteorology Period of Record: 1 Jan 75 - 31 Dec 75
Veg: $\quad 7.4 \mathrm{E} 6 \mathrm{ki}$ logram

MiTk: $5.7 \mathrm{E} 7$ liter

Meat: 5.0E7 kilogram

0.9

0.7

SITE-SPECIFIC DATA - WATERBORNE PATHWAYS via PARR RES. and BROAD RIVER

Average River Flow

at Site: $6,000 \mathrm{ft}^{3} / \mathrm{s}$

Drinking Water:

Exposed Population: 130,000

Dilution Factor: 1

Fish:

EdibTe Harvest: (a)

DiTution Factor: 1

(a) Average individual consumption rate of $2.2 \mathrm{~kg} / \mathrm{yr}$ as given in the FES (1973) used in lieu of catch data. 


\section{POPULATION DOSE-COMMITMENT ESTIMATES AND}

AVERAGE INDIVIDUAL DOSE-COMMITMENT HISTOGRAM FOR

\section{SUMMER}

Dose Commitments (person-rem) from Waterborne Pathways

\begin{tabular}{|c|c|c|c|c|c|}
\hline & Total Body & GI-LII & Thyroid & Bone & Liver \\
\hline $\begin{array}{l}\text { Infant } \\
\text { Child } \\
\text { Teen } \\
\text { Adult }\end{array}$ & $\begin{array}{l}6.7 \mathrm{E}-03 \\
1.1 \mathrm{E}-01 \\
5.2 \mathrm{E}-02 \\
3.7 \mathrm{E}-01\end{array}$ & $\begin{array}{l}6.9 \mathrm{E}-03 \\
6.7 \mathrm{E}-01 \\
1.3 \mathrm{E}+00 \\
1.1 \mathrm{E}+01\end{array}$ & $\begin{array}{l}6.7 \mathrm{E}-03 \\
6.7 \mathrm{E}-02 \\
2.5 \mathrm{E}-02 \\
2.0 \mathrm{E}-01\end{array}$ & $\begin{array}{l}2.3 \mathrm{E}-04 \\
9.8 \mathrm{E}-03 \\
5.3 \mathrm{E}-03 \\
3.3 \mathrm{E}-02\end{array}$ & $\begin{array}{l}5.4 \mathrm{E}-03 \\
9.4 \mathrm{E}-02 \\
5.6 \mathrm{E}-02 \\
3.9 \mathrm{E}-01\end{array}$ \\
\hline TOTAL & $5.4 \mathrm{E}-01$ & $1.3 \mathrm{E}+01$ & $3.0 \mathrm{E}-01$ & $4.8 E-02$ & $5.5 \mathrm{E}-01$ \\
\hline
\end{tabular}

Dose Commitments (person-rem) from Airborne Pathways Total Body GI-LII Thyroid Bone Liver $\underline{\text { Lung }}$

$\begin{array}{lllllll}\text { Infant } & 9.5 \mathrm{E}-06 & 9.5 \mathrm{E}-06 & 1.0 \mathrm{E}-05 & 8.8 \mathrm{E}-06 & 9.5 \mathrm{E}-06 & 9.8 \mathrm{E}-06 \\ \text { Child } & 1.1 \mathrm{E}-04 & 1.1 \mathrm{E}-04 & 1.1 \mathrm{E}-04 & 9.8 \mathrm{E}-05 & 1.1 \mathrm{E}-04 & 1.1 \mathrm{E}-04 \\ \text { Teen } & 7.9 \mathrm{E}-05 & 7.9 \mathrm{E}-05 & 8.0 \mathrm{E}-05 & 7.2 \mathrm{E}-05 & 7.9 \mathrm{E}-05 & 8.5 \mathrm{E}-05 \\ \text { Adult } & 4.8 \mathrm{E}-04 & 4.8 \mathrm{E}-04 & 4.8 \mathrm{E}-04 & 4.3 \mathrm{E}-04 & 4.8 \mathrm{E}-04 & 5.0 \mathrm{E}-04 \\ \text { TOTAL } & 6.7 \mathrm{E}-04 & 6.7 \mathrm{E}-04 & 6.8 \mathrm{E}-04 & 6.1 \mathrm{E}-04 & 6.7 \mathrm{E}-04 & 7.1 \mathrm{E}-04\end{array}$

Production/Consumption factors:

Produce: $<1 \quad$ Mi $1 \mathrm{k}:<1 \quad$ Meat: $<1$

Fraction of Population Receiving an Average Individual Total-Body Dose Commitment from Airborne Pathways

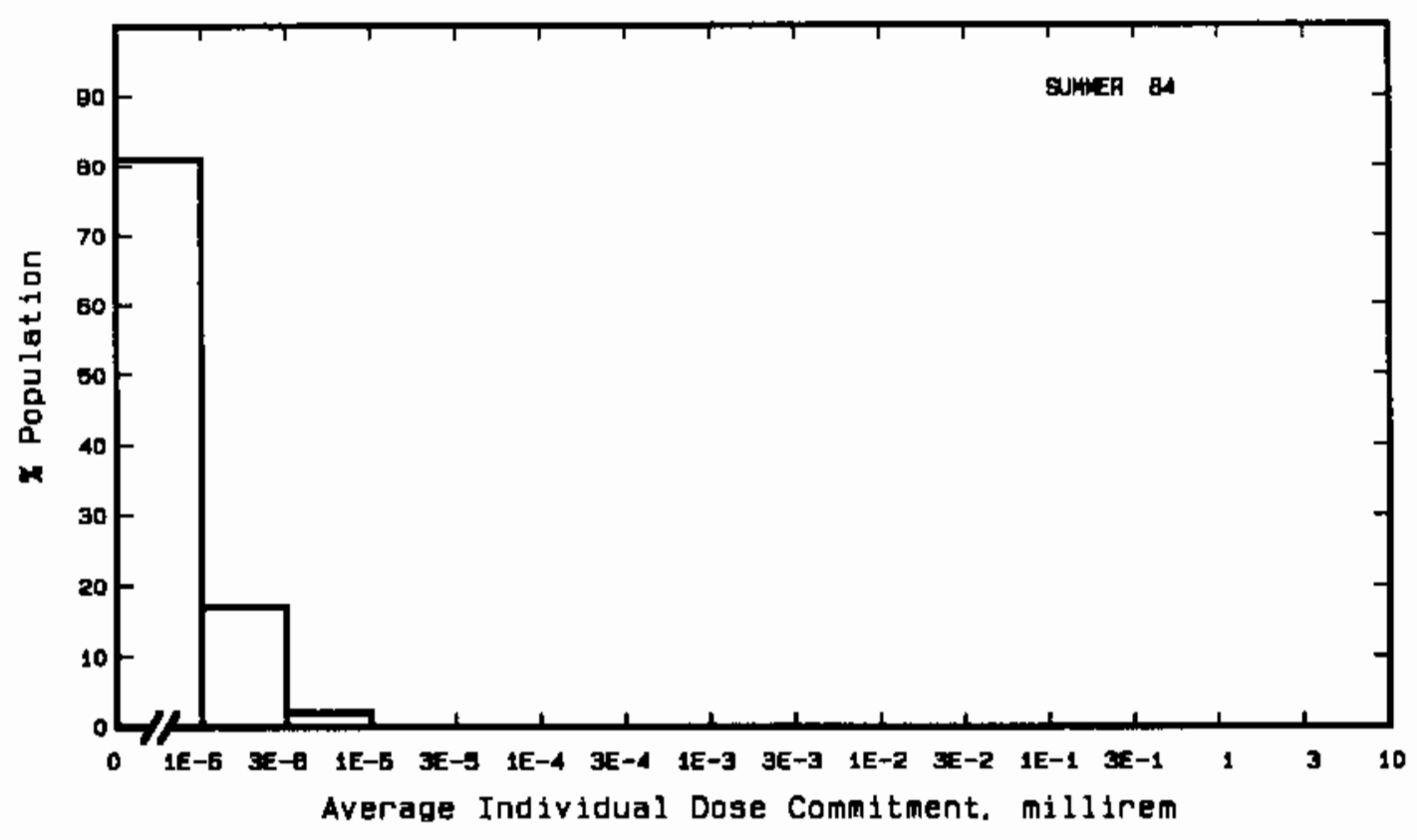


Site: SURRY

SURRY COUNTY, VIRGINIA

Location: N $37.1656^{\circ} \quad$ W $76.6983^{\circ}$

POPULATION DATA

Total Population Within 2-to-80-km Region: 1.8E6

Major Metropolitan Centers Within Region:

Center Population Location

Norfolk-Virginia Beach-Portsmouth SMSA $\quad 820,000 \quad 50 \mathrm{~km}$ SE

Richmond SMSA $\quad 650,000 \quad 77 \mathrm{~km}$ WWW

Newport News-Hampton SMSA $\quad 370,000 \quad 33 \mathrm{~km}$ ESE

Petersburg-Colonial Heights-Hopewell SMSA 130,000 $63 \mathrm{~km}$ W

Williamsburg

$10,000 \quad 12 \mathrm{~km} \quad \mathrm{~N}$

SITE-SPECIFIC DATA - AIRBORNE PATHWAYS

Average Annual State Production

of Crops and Animal Products

In $80-\mathrm{km}$ Radius Circle

Regional Productivity Factor:

Animal Grazing Factor:

Meteorology Period of Record: 3 MAR $74-2$ MAR 75 Recovery: 91\%
Veg: $3.5 E 7$ kilogram

Milk: $1.5 \mathrm{~EB}$ liter

Meat: $7.4 \mathrm{E} 7 \mathrm{kilogram}$

0.8

0.7

SITE-SPECIFIC DATA - WATERBORNE PATHWAYS via JAMES RIVER ESTUARY

Average River Flow at Site: $25,000 \mathrm{ft}^{3} / \mathrm{s}^{(\mathrm{a})}$

Fish:

Edible Harvest: $6.0 \mathrm{E} 5 \mathrm{~kg} / \mathrm{yr}$ Dilution Factor: 1

Invertebrates: ${ }^{(b)}$

EdibTe Harvest: $1.1 \mathrm{E} 6 \mathrm{~kg} / \mathrm{yr}$ Dilution Factor: 1

(a) Flow includes fresh water river flow and saline "mixing flow" of estuary as given in FES (1972).

(b) Environment primarily salt water so invertebrates considered in Tieu of drinking water. 
POPULATION DOSE-COMMITMENT ESTIMATES AND

AVERAGE INDIVIDUAL DOSE-COMMITMENT HISTOGRAM FOR

SURRY 1 AND 2

Dose Commitments (person-rem) from Waterborne Pathways

Total Body GI-LII Thyroid Bone Liver

$\begin{array}{llllll}\text { Infant } & 0.0 \mathrm{E}+00 & 0.0 \mathrm{E}+00 & 0.0 \mathrm{E}+00 & 0.0 \mathrm{E}+00 & 0.0 \mathrm{E}+00 \\ \text { ChiTd } & 1.7 \mathrm{E}-01 & 2.8 \mathrm{E}-01 & 4.0 \mathrm{E}-01 & 3.0 \mathrm{E}-01 & 3.2 \mathrm{E}-01 \\ \text { Teen } & 1.6 \mathrm{E}-01 & 5.7 \mathrm{E}-01 & 2.8 \mathrm{E}-01 & 1.7 \mathrm{E}-01 & 2.7 \mathrm{E}-01 \\ \text { Adult } & 1.3 \mathrm{E}+00 & 5.1 \mathrm{E}+00 & 1.8 \mathrm{E}+00 & 9.9 \mathrm{E}-01 & 1.6 \mathrm{E}+00 \\ \text { TOTAL } & 1.6 \mathrm{E}+00 & 5.9 \mathrm{E}+00 & 2.5 \mathrm{E}+00 & 1.5 \mathrm{E}+00 & 2.2 \mathrm{E}+00\end{array}$

Dose Commitments (person-rem) from Airborne Pathways

Total Body GI-LII Thyroid Bone Liver $\underline{\text { Lung }}$

$\begin{array}{lllllll}\text { Infant } & 4.5 \mathrm{E}-03 & 4.4 \mathrm{E}-03 & 4.1 \mathrm{E}-02 & 4.6 \mathrm{E}-03 & 4.9 \mathrm{E}-03 & 4.9 \mathrm{E}-03 \\ \text { Child } & 5.1 \mathrm{E}-02 & 5.1 \mathrm{E}-02 & 2.7 \mathrm{E}-01 & 5.0 \mathrm{E}-02 & 5.4 \mathrm{E}-02 & 5.6 \mathrm{E}-02 \\ \text { Teen } & 3.7 \mathrm{E}-02 & 3.7 \mathrm{E}-02 & 1.3 \mathrm{E}-01 & 3.5 \mathrm{E}-02 & 3.8 \mathrm{E}-02 & 4.4 \mathrm{E}-02 \\ \text { Adult } & 2.2 \mathrm{E}-01 & 2.2 \mathrm{E}-01 & 5.7 \mathrm{E}-01 & 2.1 \mathrm{E}-01 & 2.3 \mathrm{E}-01 & 2.4 \mathrm{E}-0 \mathrm{I} \\ \text { TOTAL } & 3.2 \mathrm{E}-01 & 3.1 \mathrm{E}-01 & 1.0 \mathrm{E}+00 & 3.0 \mathrm{E}-01 & 3.2 \mathrm{E}-01 & 3.5 \mathrm{E}-01\end{array}$

Production/Consumption factors:

Produce: <1 Milk: <1 Meat: <1

Fraction of Population Receiving an Average Individual

Total-Body Dose Commitment from Airborne Pathways

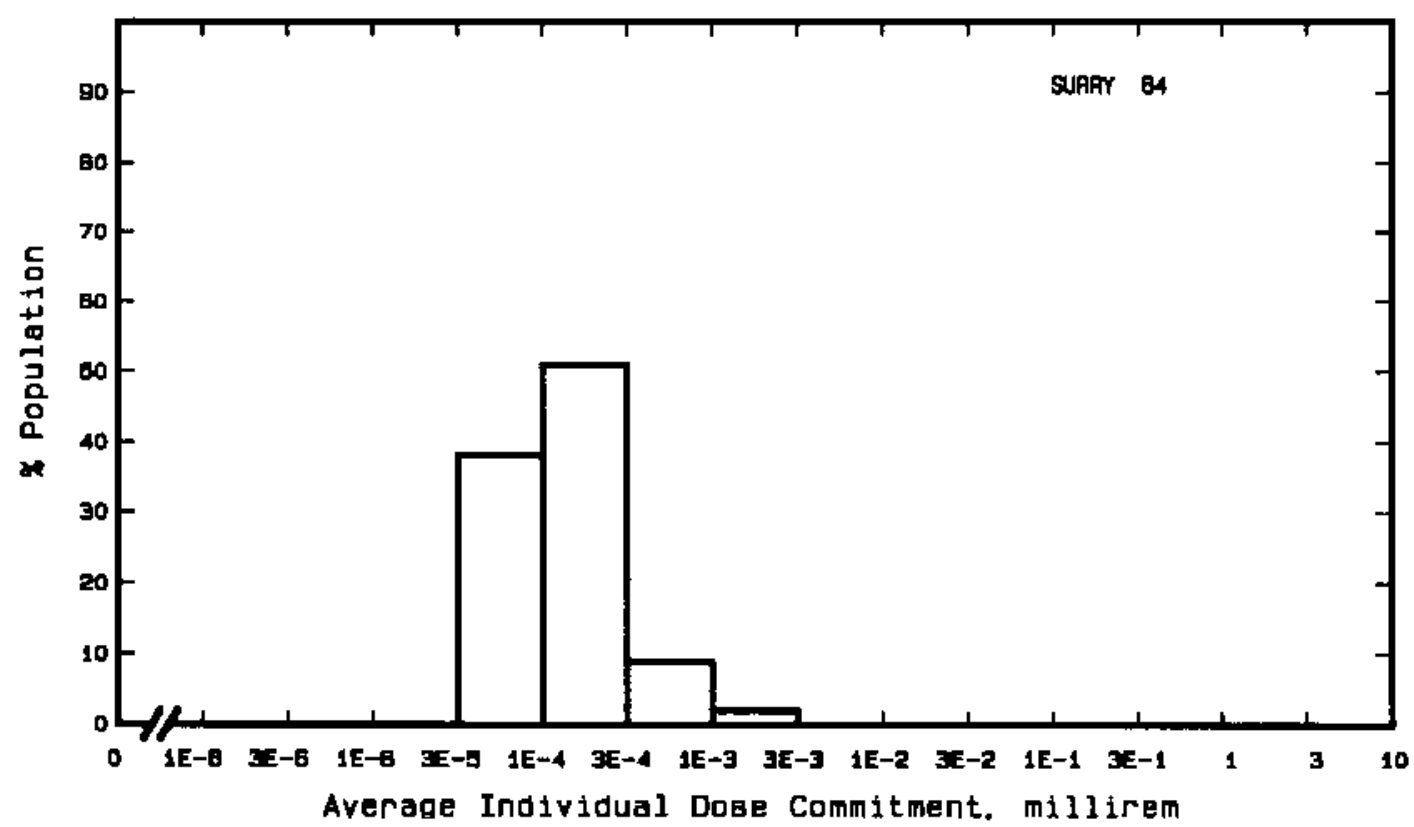


Site: SUSQUEHANNA

BERWICK, PENNSYLVANIA

Location:

N $41.1000^{\circ}$

W $76.1500^{\circ}$

POPULATION DATA

Total Population Within 2-to-80-km Region: $1.5 E 6$

Major Metropolitan Centers Within Region:

\begin{tabular}{lcccc} 
Center & Population & \multicolumn{3}{c}{ Location } \\
\cline { 2 - 6 } Williamsport - SMSA & 118,000 & $73 \mathrm{~km}$ & WNW \\
Allentown & 100,000 & $79 \mathrm{~km}$ & SE \\
Scranton & 88,000 & $53 \mathrm{~km}$ & NE \\
Wilkes-Barre & 52,000 & $28 \mathrm{~km}$ & $\mathrm{NE}$ \\
Hazleton & 27,000 & $21 \mathrm{~km}$ & SE
\end{tabular}

SITE-SPECIFIC DATA - AIRBORNE PATHWAYS

Average Annual State Production

of Crops and Animal Products

In 80-km Radius Circle

Regional Productivity Factor:

Animal Grazing Factor:

Meteorology Period of Record: 1 Jan $75-31$ Dec 75 Recovery: 98\%

SITE-SPECIFIC DATA - WATERBORNE PATHWAYS via SUSQUEHANNA RIVER

Average River Flow

at Site: $13,000 \mathrm{ft}^{3} / \mathrm{s}$

Drinking Water:

Exposed Poputation: 100,000

Dilution Factor: 1

Fish:

Edible Harvest: (a)

Dilution Factor: 0.1

(a) No fish catch data given in FES (1973), so generic consumption rates used (Table A.1).

(b) Ten percent of population consumes fish from river. 
POPULATION DOSE-COMMITMENT ESTIMATES AND

AVERAGE INDIVIDUAL DOSE-COMMITMENT HISTOGRAM FOR

\section{SUSQUEHANNA}

Dose Commitments (person-rem) from Waterborne Pathways

Total Body GI-LII Thyroid Bone Liver

$\begin{array}{llllll}\text { Infant } & 9.7 \mathrm{E}-05 & 9.8 \mathrm{E}-05 & 8.1 \mathrm{E}-05 & 5.4 \mathrm{E}-06 & 8.9 \mathrm{E}-05 \\ \text { Child } & 1.3 \mathrm{E}-03 & 1.7 \mathrm{E}-03 & 8.9 \mathrm{E}-04 & 7.4 \mathrm{E}-05 & 1.2 \mathrm{E}-03 \\ \text { Teen } & 5.4 \mathrm{E}-04 & 1.6 \mathrm{E}-03 & 3.4 \mathrm{E}-04 & 3.0 \mathrm{E}-05 & 6.2 \mathrm{E}-04 \\ \text { Adult } & 4.0 \mathrm{E}-03 & 1.5 \mathrm{E}-02 & 2.8 \mathrm{E}-03 & 1.9 \mathrm{E}-04 & 4.6 \mathrm{E}-03 \\ \text { TOTAL } & 5.9 \mathrm{E}-03 & 1.8 \mathrm{E}-02 & 4.2 \mathrm{E}-03 & 3.0 \mathrm{E}-04 & 6.6 \mathrm{E}-03\end{array}$

Oose Conmitments (person-rem) from Airborne Pathways

Total Body GI-LII Thyroid Bone Liver Lung

Infant $5.9 \mathrm{E}-04 \quad 5.7 \mathrm{E}-04 \quad 1.7 \mathrm{E}-02 \quad 2.9 \mathrm{E}-04 \quad 6.2 \mathrm{E}-04 \quad 5.8 \mathrm{E}-04$

Child 7.4E-03 7.3E-03 $1.0 \mathrm{E}-01 \quad 3.0 \mathrm{E}-03 \quad 7.5 \mathrm{E}-03 \quad 7.4 \mathrm{E}-03$

Teen $\quad 5.0 \mathrm{E}-03 \quad 4.9 \mathrm{E}-03 \quad 4.2 \mathrm{E}-02 \quad 2.1 \mathrm{E}-03 \quad 5.0 \mathrm{E}-03 \quad 5.1 \mathrm{E}-03$

Adult 2.8E-02 2.8E-02 $1.5 \mathrm{E}-01 \quad 1.2 \mathrm{E}-02 \quad 2.8 \mathrm{E}-02 \quad 2.8 \mathrm{E}-02$

TOTAL 4.1E-02 4.1E-02 3.1E-01 1.8E-02 4.1E-02 $4.2 \mathrm{E}-02$

Production/Consumption factors:

Produce: <1 Milk: $2.4 \quad$ Meat: <1

Fraction of Population Receiving an Average Individual

Total-Body Oose Commitment from Airborne Pathways

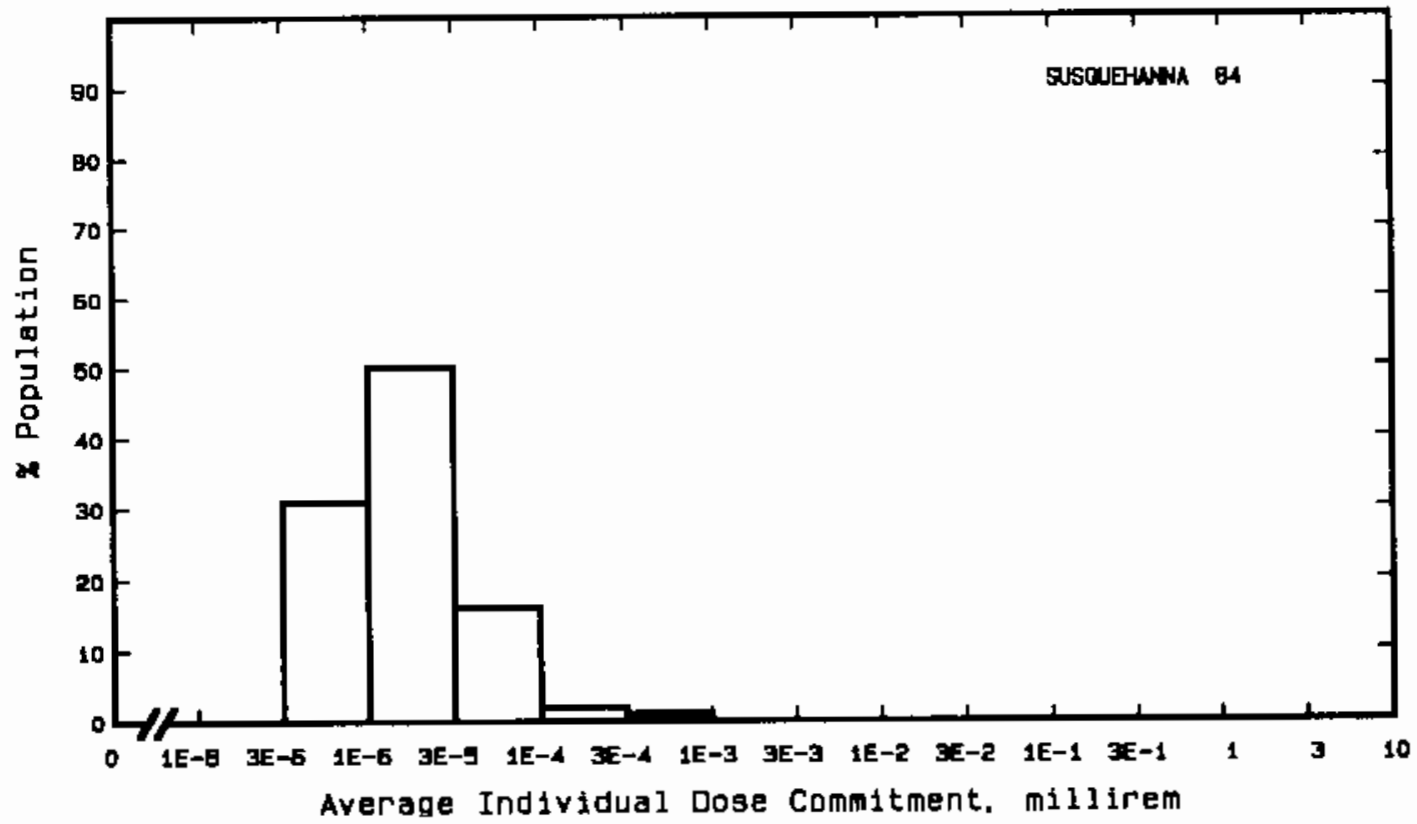


Site: THREE MILE ISLAND

THREE Mile ISLAND, PENnSylvania

Location: $\quad$ N $40.1531^{\circ}$ W $76.7250^{\circ}$

POPULATION DATA

Total Population Within 2-to-80-km Region: 2.1E6

Major Metropolitan Centers Within Region:

\begin{tabular}{lcccc}
\multicolumn{1}{c}{ Center } & Population & \multicolumn{2}{c}{ Location } \\
\cline { 5 - 6 } Harrisburg SMSA & 450,000 & & $18 \mathrm{~km}$ & NW \\
York SMSA & 380,000 & $21 \mathrm{~km}$ & S \\
Lancaster SMSA & 360,000 & $38 \mathrm{~km}$ & ESE \\
Reading SMSA & 310,000 & $71 \mathrm{~km}$ & ENE \\
Lebanon & 26,000 & $33 \mathrm{~km}$ & NE
\end{tabular}

SITE-SPECIFIC DATA - AIRBORNE PATHWAYS

Average Annual State Production

of Crops and Animal Products

In 80-km Radius Circle

Regional Productivity Factor:

Animal Grazing Factor:
Veg: $5.3 E 7$ kilogram

Mi ik: 5.3E8 liter

Meat: $5.4 \mathrm{E} 7 \mathrm{ki}$ logram

1

0.5

Meteorology Period of Record: 1 OCT 72 - 30 SEP 73 Recovery: 80\%

SITE-SPECIFIC DATA - WATERBORNE PATHWAYS via SUSQUEHANNA RIVER

Average River Flow

at Site: $34,000 \mathrm{ft}^{3} / \mathrm{s}$

Drinking Water:

Exposed Popslation: 230,000

Dilution Factor: 1

Fish:

Edible Harvest: (a) $\mathrm{kg}(\mathrm{kr})$
Dilution Factor: $0.025(\mathrm{~s})$

(a) No fish catch data given in FES (1972), so generic consumption rates used (Table A-I).

(b) Ten percent of population consumes $25 \%$ of their fish from river according to FES. 


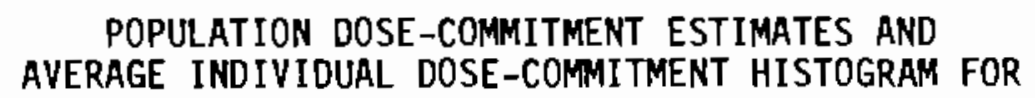

THREE MILE ISLAND 1 AND 2

Dose Commitments (person-rem) from Waterborne Pathways

Total Body GI-LII Thyroid Bone Liver

Infant

Child

Teen

Adult

$$
\text { 2.1E-04 }
$$

2. $1 E-05$

9.8E-06

9. IE-04

2.9E-04

$4.8 \mathrm{E}-03$

3.7E-04

4. $3 \mathrm{E}-03$

$3.2 \mathrm{E}-04$

1.1E-04

2.3E-02

1.2E-02

4. $4 \mathrm{E}-02$

2.6E-03

4.2E-05

1.1E-02

$8.8 \mathrm{E}-03$

TOTAL

$5.3 \mathrm{E}-02$

$3.3 \mathrm{E}-03$

5. $2 \mathrm{E}-04$

7. OE-02

$5.2 \mathrm{E}-02$

$7.4 \mathrm{E}-02$

Dose Commitments (person-rem) from Airborne Pathways

Total Body GI-LII Thyroid Bone Liver Lung

Infant

$3.2 \mathrm{E}-04$

3.2E-04

$3.2 E-04$

3. 3E-05

$3.2 \mathrm{E}-04$

3.8E-04

Child

$4.5 \mathrm{E}-03$

$4.5 \mathrm{E}-03$

4. $5 \mathrm{E}-03$

3.7E-04

4. $5 \mathrm{E}-03$

$5.2 \mathrm{E}-03$

Teen

3. $0 \mathrm{E}-03$

3. $0 \mathrm{E}-03$

3. $0 \mathrm{E}-03$

2.7E-04

3. $0 \mathrm{E}-03$

1.6E-03

1.7E-02

4. $0 \mathrm{E}-03$

TOTAL

2.5E-02

2.5E-02

2.5E-02

2. $3 \mathrm{E}-03$

2. $5 E-02$

2. OE-02

3. $0 \mathrm{E}-02$

Production/Consumption factors:

Produce: <1

Milk: 2.0

Meat: <1

Fraction of Population Receiving an Average Individual

Total-Body Dose Commitment from Airborne Pathways

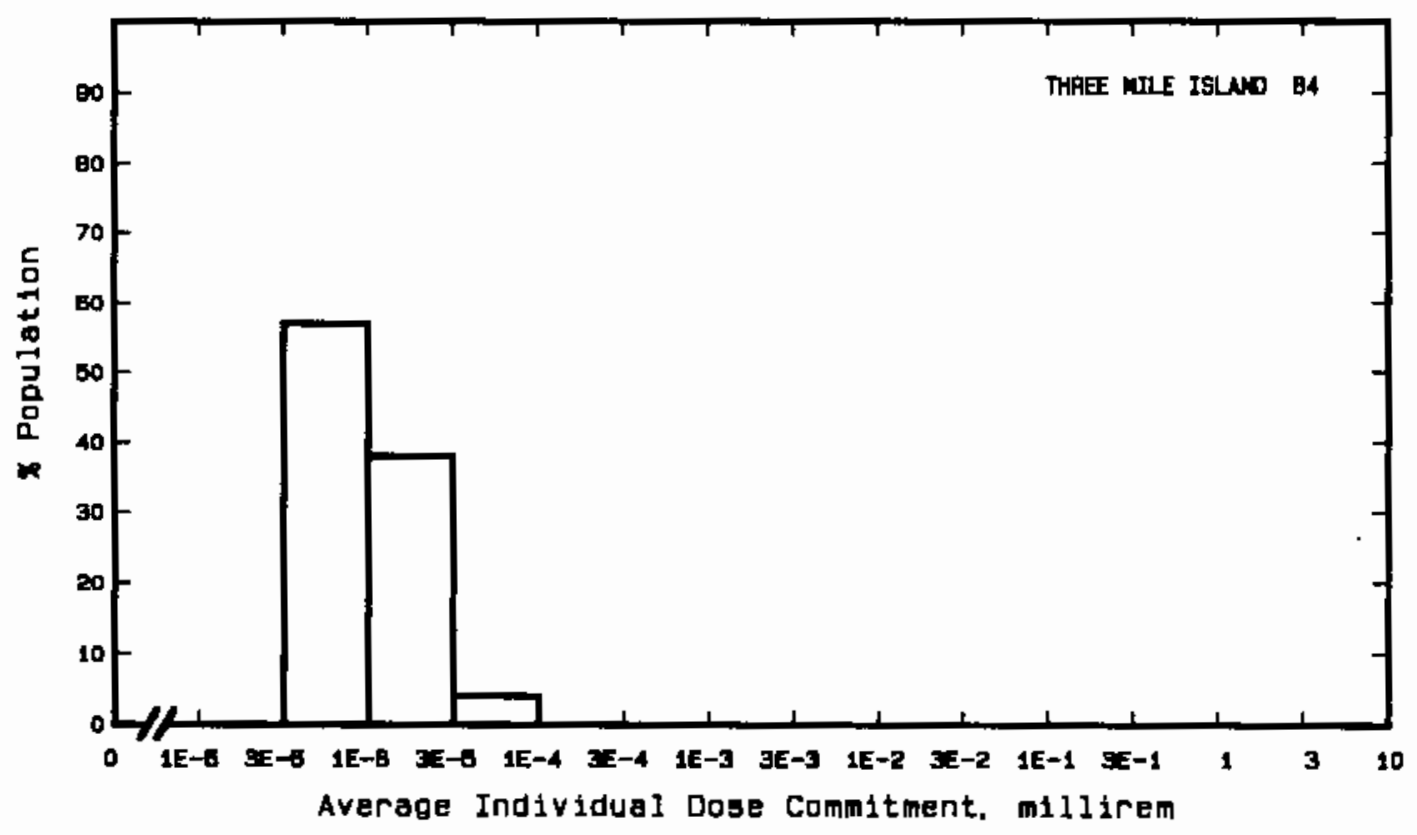

Note: Doses include releases from the TMI $2 /$ Epicore. 
Site: TROJAN

PRESCOTT, OREGON

Location: $\quad N 46.0408^{\circ} \quad$ W $122.8844^{\circ}$

POPULATION DATA

Total Population Within 2-to-80-km Region: I.5E6

Major Metropolitan Centers Within Region:

\begin{tabular}{lrrrr}
\multicolumn{1}{c}{ Center } & Population & \multicolumn{2}{c}{ Location } \\
Portland SMSA & $1,300,000$ & & $60 \mathrm{~km}$ & SSE \\
Longview & 33,000 & $12 \mathrm{~km}$ & NNW \\
Astoria & 14,000 & $72 \mathrm{~km}$ & WNW \\
Forest Grove & 13,000 & $58 \mathrm{~km}$ & SSW \\
Centralia & 12,000 & $75 \mathrm{~km}$ & $\mathrm{~N}$
\end{tabular}

SITE-SPECIFIC DATA - AIRBORNE PATHWAYS

Average Annual State Production

Veg: $6.4 E 7$ kiTogram

Of Crops and Animal Products

Milk: 3.7 E7 Titer

In 80-km Radius Circle

Meat: $2.6 E 7 \mathrm{kiTogram}$

Regional Productivity Factor:

Animal Grazing Factor:

0.9

0.75

Meteorology Period of Record: 1 SEP 71 - 31 AUG 74 Recovery: 90\%

SITE-SPECIFIC DATA - WATERBORNE PATHWAYS via COLUMBIA RIVER

Average River Flow

at Site: $2.3 \mathrm{E} 5 \mathrm{ft}^{3} / \mathrm{s}$

Drinking Water:

Exposed Population: $550^{(a)}$

Dilution Factor: 1

Fish:

Edible Harvest: $1.0 \mathrm{E} 6 \mathrm{~kg} / \mathrm{yr}$

Dilution Factor: I

(a) Population of Rainier divided by 4 , since residents only there for $25 \%$ of the year. 


\section{POPULATION DOSE-COMMITMENT ESTIMATES AND \\ AVERAGE INDIVIDUAL DOSE-COMMITMENT HISTOGRAM FOR}

TROJAN

Dose Commitments (person-rem) from Waterborne Pathways

\begin{tabular}{|c|c|c|c|c|c|}
\hline & Tota T Body & GI-LII & Thyroid & Bone & Liver \\
\hline $\begin{array}{l}\text { Infant } \\
\text { Child } \\
\text { Teen } \\
\text { Adult }\end{array}$ & $\begin{array}{l}4.5 \mathrm{E}-07 \\
9.1 \mathrm{E}-04 \\
1.6 \mathrm{E}-03 \\
1.7 \mathrm{E}-02\end{array}$ & $\begin{array}{l}4.8 \mathrm{E}-07 \\
3.1 \mathrm{E}-03 \\
6.5 \mathrm{E}-03 \\
5.6 \mathrm{E}-02\end{array}$ & $\begin{array}{l}6.3 \mathrm{E}-07 \\
5.6 \mathrm{E}-05 \\
4.0 \mathrm{E}-05 \\
2.8 \mathrm{E}-04\end{array}$ & $\begin{array}{l}4.9 \mathrm{E}-07 \\
4.8 \mathrm{E}-03 \\
2.9 \mathrm{E}-03 \\
1.7 \mathrm{E}-02\end{array}$ & $\begin{array}{l}4.8 \mathrm{E}-07 \\
4.9 \mathrm{E}-03 \\
4.1 \mathrm{E}-03 \\
2.4 \mathrm{E}-02\end{array}$ \\
\hline TOTAL & $2.0 \mathrm{E}-02$ & $6.6 \mathrm{E}-02$ & $3.8 \mathrm{E}-04$ & $2.5 E-02$ & $3.3 \mathrm{E}-02$ \\
\hline
\end{tabular}

Dose Commitments (person-rem) from Airborne Pathways

\section{Total Body GI-LII Thyroid Bone Liver}

$\begin{array}{lllllll}\text { Infant } & 6.2 \mathrm{E}-04 & 6.2 \mathrm{E}-04 & 1.5 \mathrm{E}-03 & 5.1 \mathrm{E}-04 & 6.2 \mathrm{E}-04 & 6.7 \mathrm{E}-04 \\ \text { Child } & 9.4 \mathrm{E}-03 & 8.8 \mathrm{E}-03 & 1.8 \mathrm{E}-02 & 8.9 \mathrm{E}-03 & 8.8 \mathrm{E}-03 & 9.5 \mathrm{E}-03 \\ \text { Teen } & 6.6 \mathrm{E}-03 & 6.4 \mathrm{E}-03 & 1.1 \mathrm{E}-02 & 5.7 \mathrm{E}-03 & 6.4 \mathrm{E}-03 & 7.2 \mathrm{E}-03 \\ \text { Adult } & 3.9 \mathrm{E}-02 & 3.8 \mathrm{E}-02 & 5.6 \mathrm{E}-02 & 3.2 \mathrm{E}-02 & 3.8 \mathrm{E}-02 & 4.1 \mathrm{E}-02 \\ \text { TOTAL } & 5.6 \mathrm{E}-02 & 5.4 \mathrm{E}-02 & 8.6 \mathrm{E}-02 & 4.7 \mathrm{E}-02 & 5.4 \mathrm{E}-02 & 5.8 \mathrm{E}-02\end{array}$

Production/Consumption factors:

Produce: <1 Milk: <1 Meat: <1

Fraction of Population Receiving an Average Individual

Total-Bady Dose Commitment from Airborne Patnways

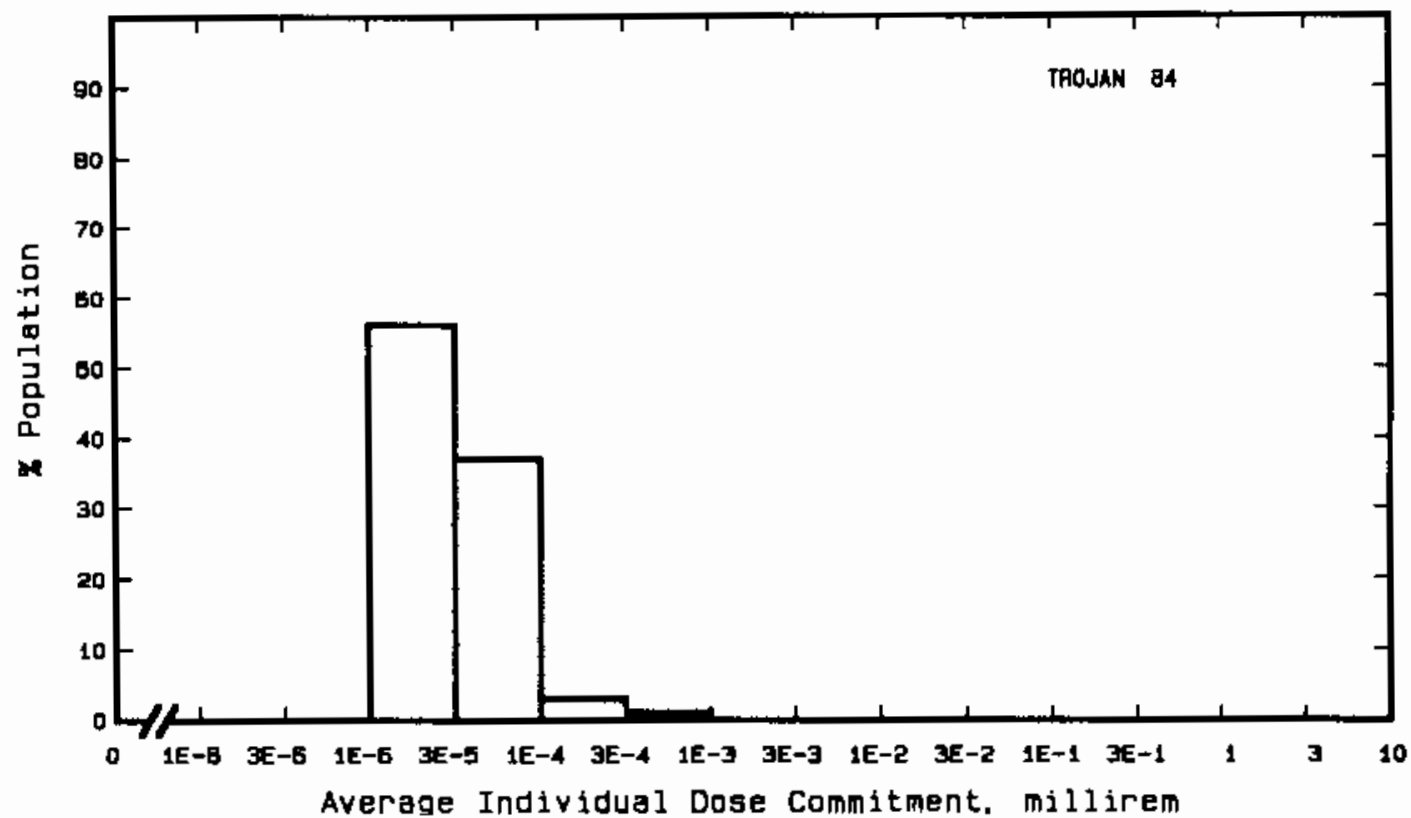


Site: TURKEY POINT

DADE COUNTY, FLORIDA

Location: N $25.4350^{\circ}$ W $80.33 \div 4^{\circ}$

POPULATION DATA

Total Population Within 2-to-80-km Region: 2.6E6

Major Metropolitan Centers Within Region:

Center Population Location

Miami SMSA

$1,700,000 \quad 41 \mathrm{~km} \quad$ NNE

Fort Lauderdale-Hollywood SMSA $1,100,000 \quad 79 \mathrm{~km}$ NNE Homes tead

Upper Keys Division

22,000

16,000

$16 \mathrm{~km}$

$42 \mathrm{~km} \mathrm{~S}$

SITE-SPECIFIC DATA - AIRBORNE PATHWAYS

Average Annual State Production

$0 f$ Crops and Animal Products

In 80-km Radius Circte

Regional Productivity Factor:

Animal Grazing Factor:

Meteorology Period of Record: 1 JAN 73 - 31 DEC 73 Recovery: 98\%
Veg: $2.8 E 7$ kilogram

Mi ik: 1.1E8 liter

Meat: $7.2 E 7$ ki Iogram

0.4

1

SITE-SPECIFIC DATA - WATERBORNE PATHWAYS via BISCAYNE BAY

Fish:

Average Dilution Flow

from Plant: $3,300 \mathrm{ft}^{3} / \mathrm{s}$

Edible Harvest: (a)

Dilution Factor: 0.001

Invertebrates:

Edible Harvest: (a)

Dilution Factor: D.002

(a) No catch data given in DES (1972), so generic consumption rates used (Table A-1). 
POPULATION DOSE-COMMITMENT ESTIMATES AND

AVERAGE INDIVIDUAL DOSE-COMMITMENT HISTOGRAM FOR

TURKEY POINT 3 AND 4

Dose Commitments (person-rem) from Waterborne Pathways

Total Body GI-LII Thyroid Bone Liver

$\begin{array}{llllll}\text { Infant } & 0.0 \mathrm{E}+00 & 0.0 \mathrm{E}+00 & 0.0 \mathrm{E}+00 & 0.0 \mathrm{E}+00 & 0.0 \mathrm{E}+00 \\ \text { Child } & 3.3 \mathrm{E}-04 & 7.9 \mathrm{E}-04 & 1.5 \mathrm{E}-04 & 2.1 \mathrm{E}-04 & 4.0 \mathrm{E}-04 \\ \text { Teen } & 2.8 \mathrm{E}-04 & 1.6 \mathrm{E}-03 & 1.2 \mathrm{E}-04 & 1.3 \mathrm{E}-04 & 3.5 \mathrm{E}-04 \\ \text { Adult } & 2.1 \mathrm{E}-03 & 1.4 \mathrm{E}-02 & 8.5 \mathrm{E}-04 & 7.6 \mathrm{E}-04 & 2.2 \mathrm{E}-03 \\ \text { TOTAL } & 2.7 \mathrm{E}-03 & 1.6 \mathrm{E}-02 & 1.1 \mathrm{E}-03 & 1.1 \mathrm{E}-03 & 2.9 \mathrm{E}-03\end{array}$

Dose Commitments (person-rem) from Airborne Pathways

Total Body GI-LII Thyroid Bone Liver Lung

$\begin{array}{lllllll}\text { Infant } & 2.5 \mathrm{E}-03 & 2.5 \mathrm{E}-03 & 1.7 \mathrm{E}-02 & 2.5 \mathrm{E}-03 & 2.5 \mathrm{E}-03 & 2.7 \mathrm{E}-03 \\ \text { Child } & 2.8 \mathrm{E}-02 & 2.8 \mathrm{E}-02 & 1.1 \mathrm{E}-01 & 2.8 \mathrm{E}-02 & 2.8 \mathrm{E}-02 & 3.1 \mathrm{E}-02 \\ \text { Teen } & 2.0 \mathrm{E}-02 & 2.0 \mathrm{E}-02 & 5.2 \mathrm{E}-02 & 2.0 \mathrm{E}-02 & 2.0 \mathrm{E}-02 & 2.5 \mathrm{E}-02 \\ \text { Adult } & 1.2 \mathrm{E}-01 & 1.2 \mathrm{E}-01 & 2.3 \mathrm{E}-01 & 1.2 \mathrm{E}-01 & 1.2 \mathrm{E}-01 & 1.4 \mathrm{E}-01 \\ \text { TOTAL } & 1.7 \mathrm{E}-01 & 1.7 \mathrm{E}-01 & 4.0 \mathrm{E}+01 & 1.7 \mathrm{E}-01 & 1.7 \mathrm{E}-01 & 1.9 \mathrm{E}-01\end{array}$

Production/Consumption factors:

Produce: <1 Milk: <1 Meat: <1

Fraction of Population Receiving an Average Individual Total-body Doge Commitment from Airborne Pathways

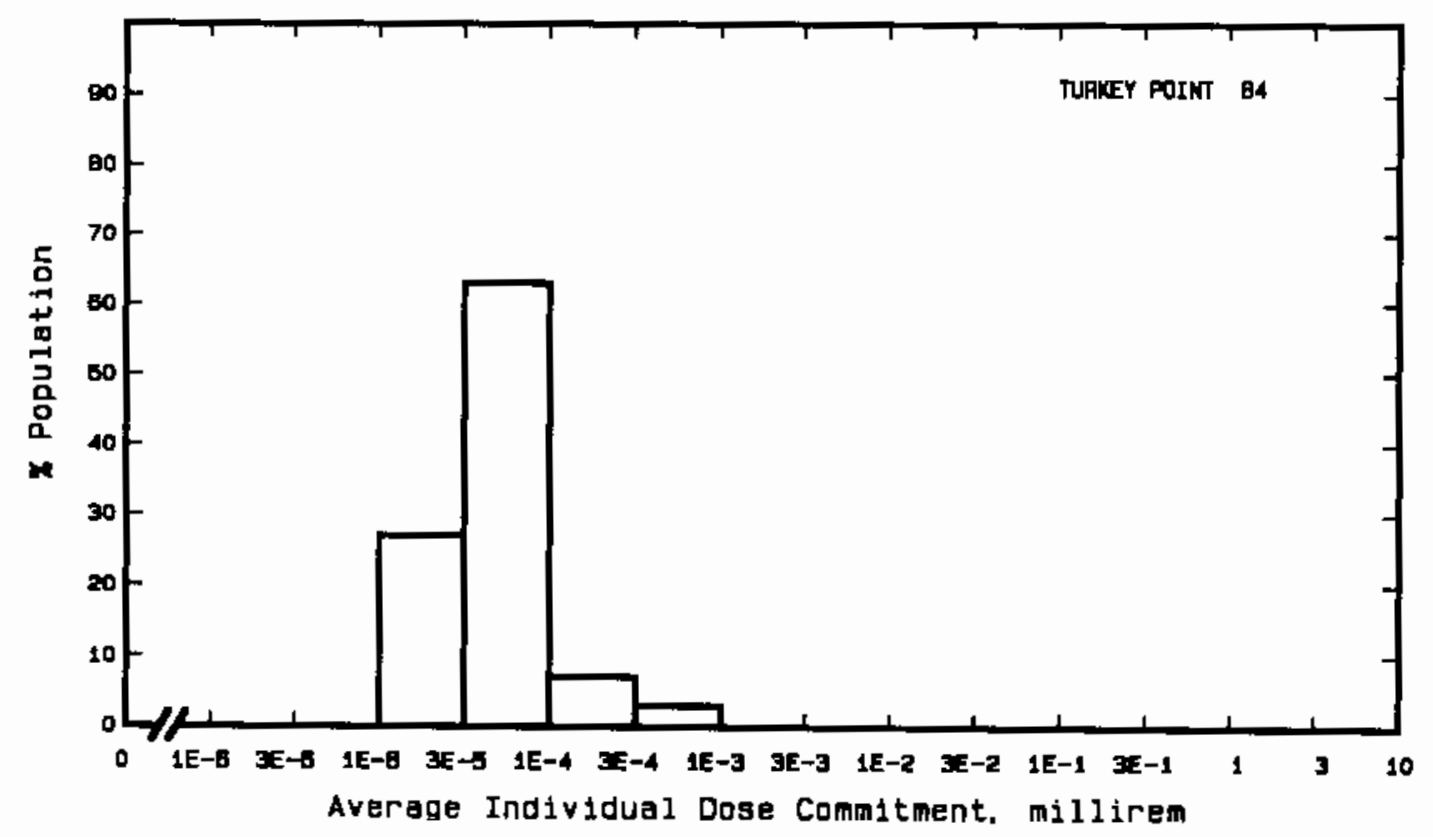


Site: VERMONT YANKEE

VERNON, VERMONT

Location: N $42.7803^{\circ} \quad$ W $72.5158^{\circ}$

POPULATION DATA

Total Population Within 2-to-80-km Region: 1.4E6

Major Metropolitan Centers Within Region:

Center Population Location

Springfield-Chicopee-Holyoke SMSA $550,000 \quad 70 \mathrm{~km} \quad \mathrm{~S}$

Worcester SMSA (1/2) $150,0010 \quad 80 \mathrm{~km}$ SE

Pittsfield

$95,010 \quad 71 \mathrm{~km} \quad \mathrm{SW}$

Fitchburg

Leominster

42,0010

37,000

Keene

22,000

$63 \mathrm{~km}$ ESE

$68 \mathrm{~km} \quad \mathrm{ESE}$

$26 \mathrm{~km} \quad \mathrm{NW}$

SITE-SPECIFIC DATA - AIRBORNE PATHWAYS

Average Annual State Production

of Crops and Anima 7 Products

In $80-\mathrm{km}$ Radius Circle

Regional Productivity Factor:

Animal Grazing Factor:

Meteorology Period of Record: 1 APR 75 - 31 MAR 76 Recovery: 97\%
Veg: 4.4E6 kilogram

MiTk: 7.3E8 liter

Meat: $2.7 \mathrm{E} 7$ kilogram

1

0.4

SITE SPECIFIC OATA - WATERBORNE PATHWAYS via CONNECTICUT RIVER at VERNON POND Average River Flow

at Site: $10,000 \mathrm{ft}^{3} / \mathrm{s}$

Orinking Water:

Exposed Poputation: None

Fish:

Edible Harvest: (a)
Dilution Factor:

(a) No radionuclides released in liquid effluents reported (Tichler, et a1. 1987). 


\section{POPULATION DOSE-COMMITMENT ESTIMATES AND \\ AVERAGE INDIVIDUAL DOSE-COMMITMENT HISTOGRAM FOR

\author{
VERMONT YANKEE
}

Dose Commitments (person-rem) from Waterborne Pathways

Total Body GI-LII Thyroid Bone Liver

Infant

Child

Teen

Adult

(No Waterborne Pathway Doses)

TOTAL

Dose Commitments (person-rem) from Airborne Pathways

\begin{tabular}{|c|c|c|c|c|c|c|}
\hline & Total Body & GI-LII & Thyroid & Bone & Liver & Lung \\
\hline $\begin{array}{l}\text { Infant } \\
\text { Child } \\
\text { Teen } \\
\text { Adult }\end{array}$ & $\begin{array}{l}1.4 \mathrm{E}-03 \\
1.6 \mathrm{E}-02 \\
1.1 \mathrm{E}-02 \\
6.7 \mathrm{E}-02\end{array}$ & $\begin{array}{l}1.4 \mathrm{E}-03 \\
1.6 \mathrm{E}-02 \\
1.1 \mathrm{E}-02 \\
6.7 \mathrm{E}-02\end{array}$ & $\begin{array}{l}3.8 \mathrm{E}-03 \\
3.1 \mathrm{E}-02 \\
1.9 \mathrm{E}-02 \\
9.8 \mathrm{E}-02\end{array}$ & $\begin{array}{l}1.2 \mathrm{E}-03 \\
1.3 \mathrm{E}-02 \\
9.7 \mathrm{E}-03 \\
5.8 \mathrm{E}-02\end{array}$ & $\begin{array}{l}1.5 \mathrm{E}-03 \\
1.6 \mathrm{E}-02 \\
1.1 \mathrm{E}-02 \\
6.7 \mathrm{E}-02\end{array}$ & $\begin{array}{l}1.6 \mathrm{E}-03 \\
1.9 \mathrm{E}-02 \\
1.4 \mathrm{E}-02 \\
7.8 \mathrm{E}-02\end{array}$ \\
\hline TTAL & $9.6 E-02$ & $9.6 \mathrm{E}-02$ & $1.5 E-01$ & 8.2E-02 & $9.6 \mathrm{E}-02$ & 1.1E-01 \\
\hline
\end{tabular}

Production/Consumption factors:

Produce: <1 Milk: $4.0 \quad$ Meat: <1

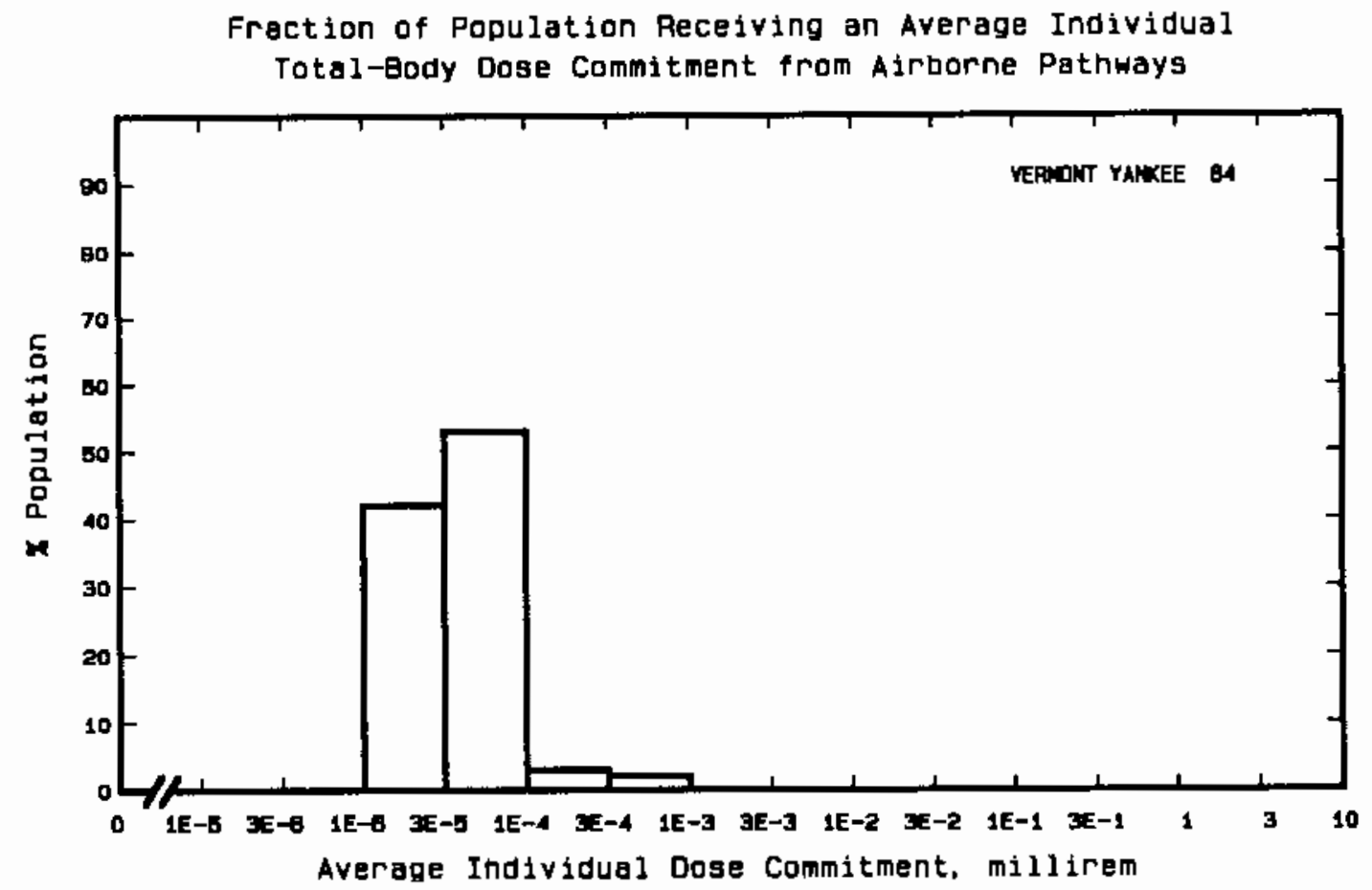


Site: WNP-2

Location:
N $46.2833^{\circ}$
RICHLAND, WASHINGTON

W $119.2916^{\circ}$

POPULATION OATA

Total PopuTation Within 2-to-80-km Region: 2.6E5

Major Metropolitan Centers Within Region:

Center Population Location

Richland $\quad 34,000 \quad 14 \mathrm{~km} \quad \mathrm{~S}$

Kennewick $\quad 35,000 \quad 26 \mathrm{~km}$ SSE

$\begin{array}{llll}\text { Pasco } & 19,000 & 28 \mathrm{~km} & \text { SSE }\end{array}$

Moses Lake $\quad 11,000 \quad 73 \mathrm{~km} \mathrm{~N}$

SITE-SPECIFIC DATA - AIRBORNE PATHWAYS

Average Annual State Production

of Crops and Anima Products

In 80-km Radius Circle

Regional Productivity Factor:

Anima Trazing Factor:
Veg: 2.1E8 kilogram

MiTk: 1.2E8 liter

Meat: $3.2 E 7$ kilogram

0.8

0.7

Meteorology Period of Record: 1 APR 75 - 31 MAR 76 Recovery: 93\%

SITE SPECIFIC OATA - WATERBORNE PATHWAYS via COLUMBIA RIVER

Average River Flow

at Site: $115,000 \mathrm{ft}^{3} / \mathrm{s}$

Drinking Water:

Exposed Population: 78,000
Dilution Factor: 3.4

Fish:

Edible Harvest: $7.5 \mathrm{E} 3 \mathrm{~kg} / \mathrm{yr}$ Dilution Factor: 1

(a) Effective factor for the cities of Richland, Kennewick, and Pasco over complete river dilution. 


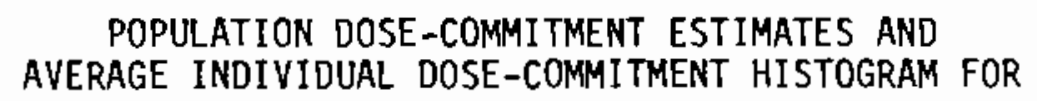

WNP -2

Dose Comitments (person-rem) from Waterborne Pathways

\begin{tabular}{|c|c|c|c|c|c|}
\hline & Total Body & $\underline{G I-L I I}$ & Thyroid & Bone & Liver \\
\hline $\begin{array}{l}\text { Infant } \\
\text { Child } \\
\text { Teen } \\
\text { Adult }\end{array}$ & $\begin{array}{l}1.2 \mathrm{E}-06 \\
1.3 \mathrm{E}-05 \\
5.0 \mathrm{E}-06 \\
4.1 \mathrm{E}-05\end{array}$ & $\begin{array}{l}1.6 \mathrm{E}-06 \\
1.7 \mathrm{E}-05 \\
8.4 \mathrm{E}-06 \\
7.0 \mathrm{E}-05\end{array}$ & $\begin{array}{l}9.5 \mathrm{E}-07 \\
1.1 \mathrm{E}-05 \\
4.1 \mathrm{E}-06 \\
3.5 \mathrm{E}-05\end{array}$ & $\begin{array}{l}9.5 \mathrm{E}-08 \\
1.3 \mathrm{E}-06 \\
4.4 \mathrm{E}-07 \\
3.2 \mathrm{E}-06\end{array}$ & $\begin{array}{l}1.3 \mathrm{E}-06 \\
1.4 \mathrm{E}-05 \\
5.7 \mathrm{E}-06 \\
4.5 \mathrm{E}-05\end{array}$ \\
\hline TOTAL & $6.0 \mathrm{E}-05$ & $9.7 \mathrm{E}-05$ & $5.0 E-05$ & $5.0 \mathrm{E}-06$ & $6.7 \mathrm{E}-05$ \\
\hline
\end{tabular}

Dose Commitments (person-rem) from Airborne Pathways

\begin{tabular}{|c|c|c|c|c|c|}
\hline Total Body & GI-LII & Thyroid & Bone & Liver & Lung \\
\hline $\begin{array}{l}3.7 \mathrm{E}-04 \\
5.8 \mathrm{E}-03 \\
3.4 \mathrm{E}-03 \\
1.9 \mathrm{E}-02\end{array}$ & $\begin{array}{l}\text { 4. } 7 \mathrm{E}-04 \\
5 . \mathrm{BE}-03 \\
5.3 \mathrm{E}-03 \\
3.0 \mathrm{E}-02\end{array}$ & $\begin{array}{l}2.6 \mathrm{E}-03 \\
2.5 \mathrm{E}-02 \\
1.2 \mathrm{E}-02 \\
5.0 \mathrm{E}-02\end{array}$ & $\begin{array}{l}5.0 \mathrm{E}-04 \\
9.3 \mathrm{E}-03 \\
4.8 \mathrm{E}-03 \\
2.4 \mathrm{E}-02\end{array}$ & $\begin{array}{l}6.4 \mathrm{E}-04 \\
7.5 \mathrm{E}-03 \\
4.3 \mathrm{E}-03 \\
2.1 \mathrm{E}-02\end{array}$ & $\begin{array}{l}\text { 7.5E-04 } \\
1.1 \mathrm{E}-02 \\
9.1 \mathrm{E}-03 \\
4.1 \mathrm{E}-02\end{array}$ \\
\hline $2.8 E-02$ & $4.2 E-02$ & 8.9E-02 & $3.8 \mathrm{E}-02$ & $3.3 \mathrm{E}-02$ & $6.1 E-02$ \\
\hline
\end{tabular}

Production/Consumption factors:

Produce: <3.3 Milk: 2.9 Meat: <1.2

Fraction of Population Receiving an Average Individual Total-Body Dose Commitment from Airborne Pathways

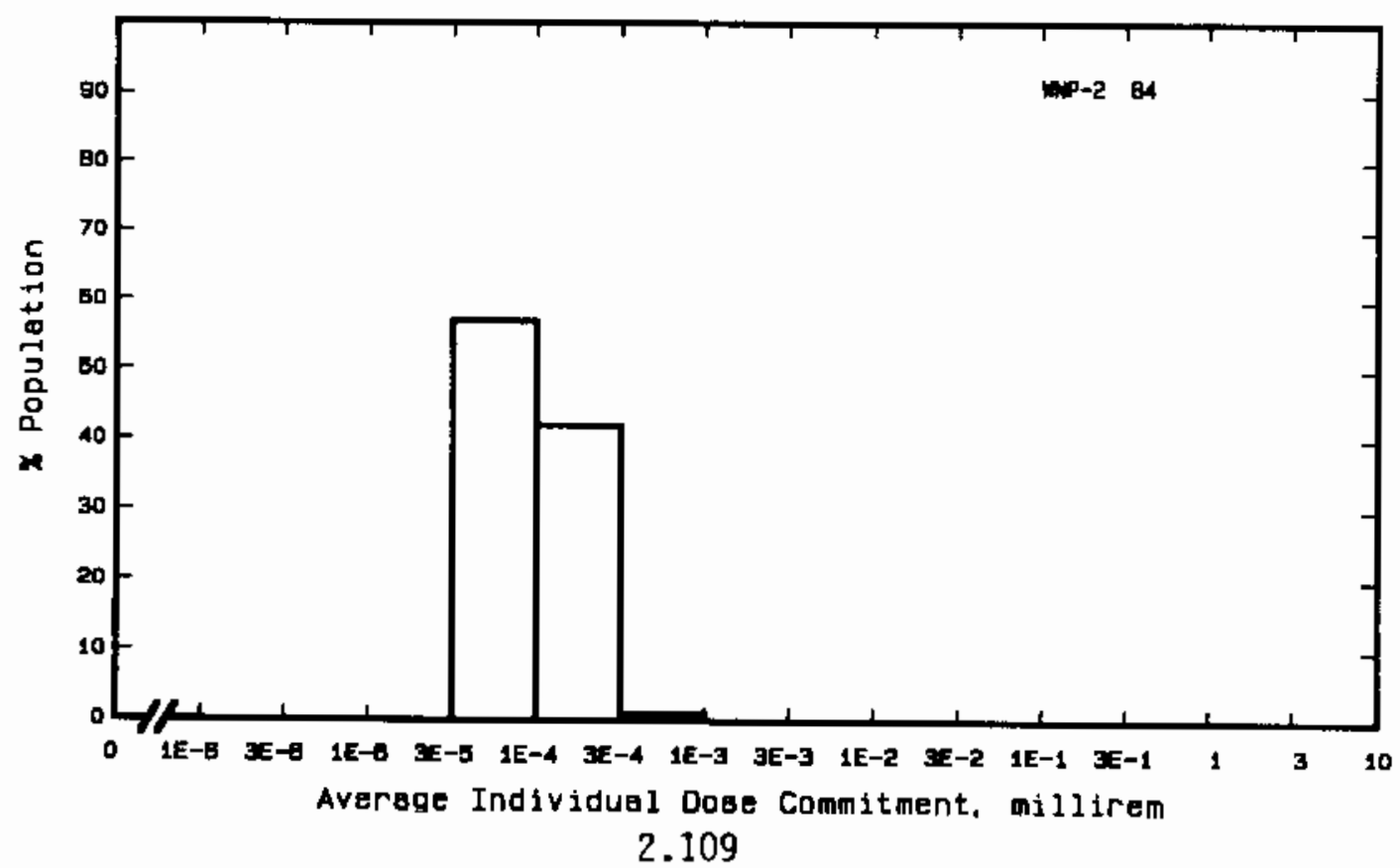


Site: YANKEE ROWE

ROWE, MASSACHUSETTS

Location: N $42.7281^{\circ}$ W $72.9239^{\circ}$

POPULATION DATA

TotaT PopuTation Within 2-to-80-km Region: 1.6E6

Major Metropolitan Centers Within Region:

Center

Population Location

Springfield-Chicopee-Holyoke SMSA

Albany

Pittsfield SMSA

Troy

530,000

100,000

91,000

Amherst

57,000

18,000

$74 \mathrm{~km} \quad \mathrm{SSE}$

$68 \mathrm{~km} \quad W$

$41 \mathrm{~km} \quad \mathrm{SW}$

$62 \mathrm{~km} \quad W$

$51 \mathrm{~km} \quad \mathrm{SE}$

SITE-SPECIFIC DATA - AIRBORNE PATHWAYS

Average Annual State Production

Veg: $2.0 \mathrm{E} 7 \mathrm{kilogram}$

of Crops and Animal Products

Milk: 2.6E8 Titer

In 80-km Radius Circle

Meat: 1.6E7 kilogram

Regional Productivity Factor:

Animal Grazing Factor:

1

0.5

Meteorology Period of Record: 1 OCT 71 - 30 SEP 72 Recovery: $94 \%$

SITE-SPECIFIC DATA - WATERBORNE PATHWAYS via DEERFIELD RIVER

Average River Flow

at Site: $570 \mathrm{ft}^{3} / \mathrm{s}$

Drinking Water:

Exposed Population: None

Fish:

Edible Harvest: (a)

DiTution Factor: $0.025^{(b)}$

(a) No catch data available, so generic consumption rates used (Table A-1).

(b) Ten percent of population obtain $25 \%$ of their fish from river. 
POPULATION OOSE-COMMITMENT ESTIMATES ANO

AVERAGE INOIVIDUAL DOSE-COMMITMENT HISTOGRAM FOR

YANKEE ROWE

Dose Commitments (person-rem) from Waterborne Pathways

Total Body GI-LII Thyroid Bone Liver

$\begin{array}{llllll}\text { Infant } & 0.0 \mathrm{E}+00 & 0.0 \mathrm{E}+00 & 0.0 \mathrm{E}+00 & 0.0 \mathrm{E}+00 & 0.0 \mathrm{E}+00 \\ \text { Child } & 1.4 \mathrm{E}-02 & 3.4 \mathrm{E}-03 & 9.1 \mathrm{E}-03 & 6.1 \mathrm{E}-02 & 6.3 \mathrm{E}-02 \\ \text { Teen } & 2.3 \mathrm{E}-02 & 3.7 \mathrm{E}-03 & 6.5 \mathrm{E}-03 & 3.7 \mathrm{E}-02 & 5.4 \mathrm{E}-02 \\ \text { Adult } & 2.4 \mathrm{E}-01 & 2.9 \mathrm{E}-02 & 4.3 \mathrm{E}-02 & 2.2 \mathrm{E}-01 & 3.2 \mathrm{E}-01 \\ \text { TOTAL } & 2.7 \mathrm{E}-01 & 3.6 \mathrm{E}-02 & 5.9 \mathrm{E}-02 & 3.1 \mathrm{E}-01 & 4.4 \mathrm{E}-01\end{array}$

Dose Commitments (person-rem) from Airborne Pathways

Total Body GI-LII Thyroid Bone Liver Lung

$\begin{array}{lllllll}\text { Infant } & 9.1 \mathrm{E}-03 & 9.1 \mathrm{E}-03 & 1.4 \mathrm{E}-02 & 3.7 \mathrm{E}-02 & 9.1 \mathrm{E}-03 & 9.1 \mathrm{E}-03 \\ \text { Child } & 6.5 \mathrm{E}-02 & 6.5 \mathrm{E}-02 & 9.5 \mathrm{E}-02 & 2.6 \mathrm{E}-01 & 6.6 \mathrm{E}-02 & 6.6 \mathrm{E}-02 \\ \text { Teen } & 2.6 \mathrm{E}-02 & 2.6 \mathrm{E}-02 & 3.9 \mathrm{E}-02 & 8.5 \mathrm{E}-02 & 2.7 \mathrm{E}-02 & 2.8 \mathrm{E}-02 \\ \text { Adult } & 1.1 \mathrm{E}-01 & 1.1 \mathrm{E}-01 & 1.5 \mathrm{E}-01 & 2.8 \mathrm{E}-01 & 1.1 \mathrm{E}-01 & 1.2 \mathrm{E}-01 \\ \text { ToTAL } & 2.1 \mathrm{E}-01 & 2.1 \mathrm{E}-01 & 3.0 \mathrm{E}-01 & 6.6 \mathrm{E}-01 & 2.1 \mathrm{E}-01 & 2.2 \mathrm{E}-01\end{array}$

Production/Consumption factors:

Produce: <1 Milk: 1.2 Meat: <1

Fraction of Population Receiving an Average Individual

Tota1-Body Dose Commitment from Airuorne Pathways

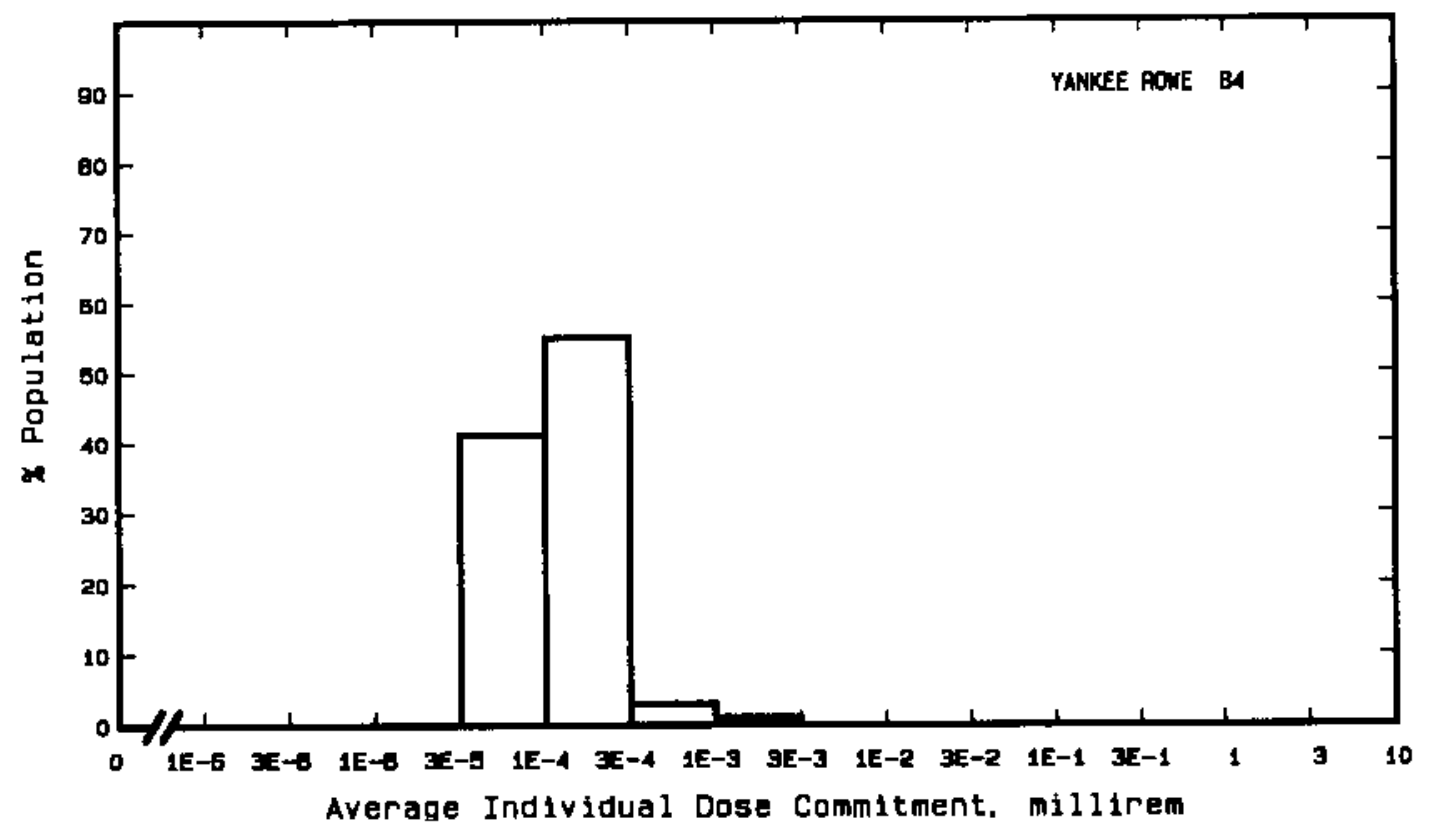


Site: ZION

ZION, ILLINOIS

Location: N $42.4456^{\circ}$ W $87.8022^{\circ}$

POPULATION DATA

Total Population Within 2-to-80-km Region: 7.2E6

Major Metropolitan Centers Within Region:

\begin{tabular}{lrrrr}
\multicolumn{1}{c}{ Center } & Population & \multicolumn{2}{c}{ Location } \\
Chicago SMSA & & & \\
Mi (2/3) & $4,700,000$ & $66 \mathrm{~km}$ & $\mathrm{~S}$ \\
Racine SMSA & $1,400,000$ & $65 \mathrm{~km}$ & $\mathrm{~N}$ \\
Kenosha SMSA & 170,000 & $30 \mathrm{~km}$ & $\mathrm{~N}$ \\
Waukesha & 120,000 & $14 \mathrm{~km}$ & $\mathrm{~N}$ \\
Wauk & 50,000 & $71 \mathrm{~km}$ & $\mathrm{NNW}$
\end{tabular}

SITE-SPECIFIC DATA - AIRBORNE PATHWAYS

Average Annual State Production

Veg: $1.1 \mathrm{E} 8 \mathrm{kilogram}$

of Crops and Animal Products

In 80-km Radius Circle

MiIk: 1.8E8 liter

Meat: $1.9 \mathrm{E} 8$ kilogram

Regional Productivity Factor:

Animal Grazing Factor:

0.5

0.5

Meteorology Period of Record: 1 JAN 74 - 3I DEC 75 Recovery: $88 \%$

SITE-SPECIFIC DATA - WATERBORNE PATHWAYS via LAKE MICHIGAN

Average Dilution Flow

from PTant: $1,500 \mathrm{ft}^{3} / \mathrm{s}$

Drinking Water:

Exposed Population: $6,809,000$

Fish:

EdibTe Harvest: $5.0 \mathrm{E} 6(\mathrm{~kg} / \mathrm{yr}$

(a) Drinking water dilution factor estimated by averaging dilution factors derived from FES (1972) suitably weighted for poputation.

(b) Dilution factor derived from FES. 
POPULATION DOSE-COMMITMENT ESTIMATES AND

AVERAGE INDIVIDUAL DOSE-COMMITMENT HISTOGRAM FOR

\author{
ZION 1 AND 2
}

Dose Commitments (person-rem) from Waterborne Pathways

Total Body GI-LII Thyroid Bone Liver

$\begin{array}{llllll}\text { Infant } & 2.2 \mathrm{E}-01 & 1.9 \mathrm{E}-01 & 4.8 \mathrm{E}+00 & 4.7 \mathrm{E}-01 & 7.6 \mathrm{E}-01 \\ \text { Chi1d } & 3.4 \mathrm{E}+00 & 2.9 \mathrm{E}+00 & 3.4 \mathrm{E}+01 & 7.5 \mathrm{E}+00 & 1.0 \mathrm{E}+01 \\ \text { Teen } & 2.6 \mathrm{E}+00 & 2.1 \mathrm{E}+00 & 1.1 \mathrm{E}+01 & 2.8 \mathrm{E}+00 & 5.3 \mathrm{E}+00 \\ \text { Adu1t } & 2.6 \mathrm{E}+01 & 1.9 \mathrm{E}+01 & 7.5 \mathrm{E}+01 & 1.7 \mathrm{E}+01 & 3.3 \mathrm{E}+01 \\ \text { TOTAL } & 3.3 \mathrm{E}+01 & 2.4 \mathrm{E}+01 & 1.2 \mathrm{E}+02 & 2.8 \mathrm{E}+01 & 4.9 \mathrm{E}+01\end{array}$

Dose Commitments (person-rem) from Airborne Pathways Total Body GI-LII Thyroid Bone Liver Lung

$\begin{array}{lllllll}\text { Infant } & 9.4 \mathrm{E}-03 & 9.3 \mathrm{E}-03 & 1.1 \mathrm{E}-02 & 8.9 \mathrm{E}-03 & 9.4 \mathrm{E}-03 & 9.8 \mathrm{E}-03 \\ \text { Child } & 1.1 \mathrm{E}-01 & 1.1 \mathrm{E}-01 & 1.3 \mathrm{E}-01 & 1.0 \mathrm{E}-01 & 1.1 \mathrm{E}-01 & 1.2 \mathrm{E}-01 \\ \text { Teen } & 8.0 \mathrm{E}-02 & 8.0 \mathrm{E}-02 & 8.9 \mathrm{E}-02 & 7.4 \mathrm{E}-02 & 8.0 \mathrm{E}-02 & 8.9 \mathrm{E}-02 \\ \text { Adult } & 4.9 \mathrm{E}-01 & 4.8 \mathrm{E}-01 & 5.2 \mathrm{E}-01 & 4.5 \mathrm{E}-01 & 4.8 \mathrm{E}-01 & 5.1 \mathrm{E}-01 \\ \text { TOTAL } & 6.9 \mathrm{E}-01 & 6.8 \mathrm{E}-01 & 7.5 \mathrm{E}-01 & 6.3 \mathrm{E}-01 & 6.8 \mathrm{E}-01 & 7.3 \mathrm{E}-01\end{array}$

Production/Consumption factors:

Produce: <1 Milk: <1 Meat: <1

Fraction of Population Receiving an Average Individual Total-Body Dose Commitment from Airborne Pathways

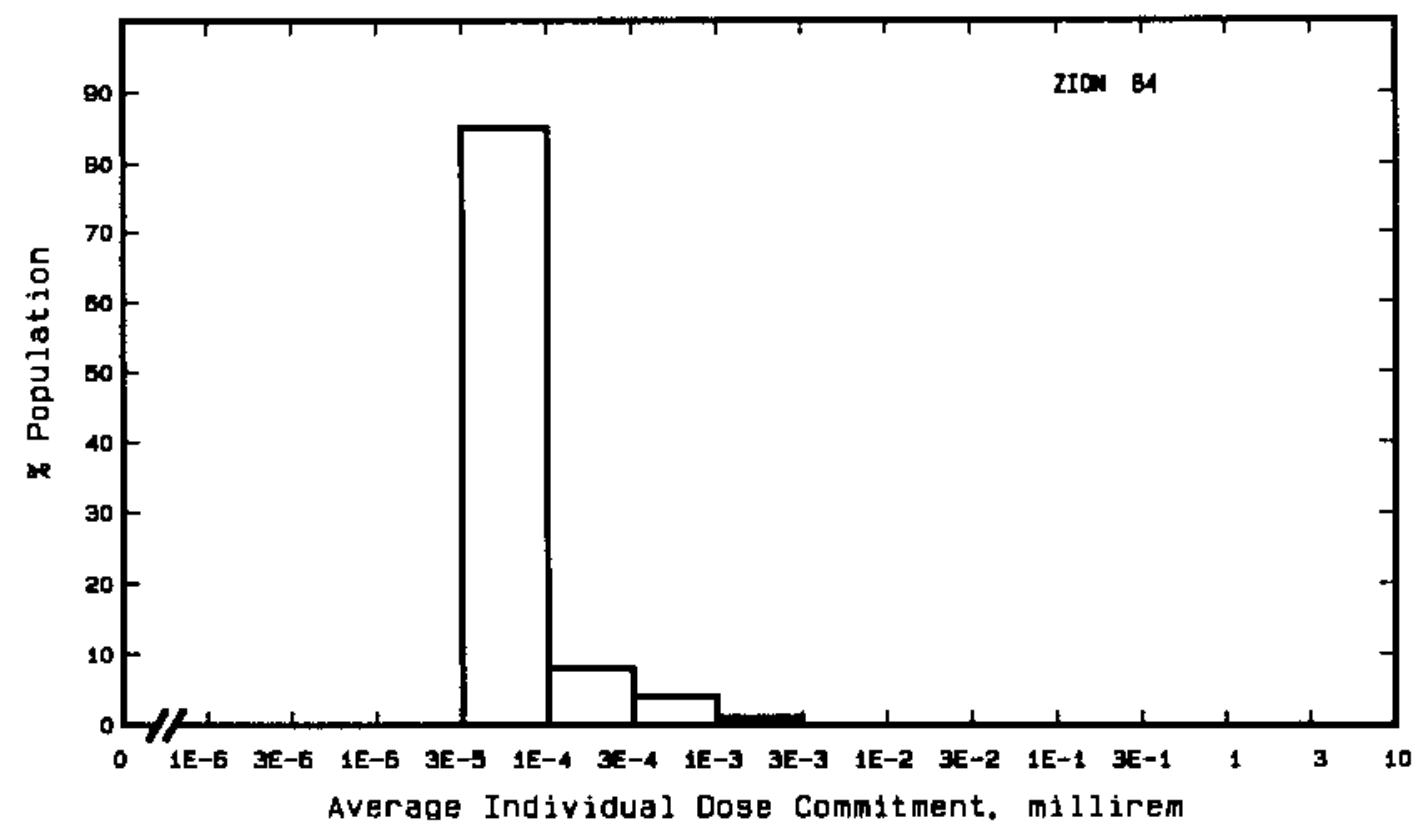





\section{REFERENCES}

Final Environmental Statement Concerning Proposed RuTe-Making Action: Numerical Guides for Design objectives and Limiting Conditions for operation to Meet the Criterion "As Low As Practicable" for Radioactive Material in Light-Water-Cooled Nuclear Power Reactor Effluents. 1973. WASH-1258, VoT. 1, Directorate of Regulatory Standards, U.S. Atomic Energy Commission, Washington, D.C.

Tichler, J., K. Norden, and J. Congemi. 1987. Radioactive Materials Released from Nuclear Power Plants, Annual Report 1984. NUREG/ CR-2907, BNL-NUREG-51581, VoT. 5, U.S. Nuclear Regulatory Commission, Washington, D.C.*

*Available for purchase from the NRC/GPO Sales Program, U.S. Nuclear Regutatory Commission, Washington, DC 20555, and the NationaT Technical Information Service, Springfield, VA 22161. 



\section{MODELS AND GENERIC PARAMETERS}

The calculational models used were primarily those given in the Nuclear Regulatory Commission's Regulatory Guide 1.109 (NRC 1977). Computer programs were written to use these models to generate population dose commitments for four age groups. The percentages of the population comprising the four age groups were $1.44 \%$, infant; $16.0 \%$, child; $11.7 \%$, teenager; and 70.9\%, aduTt (Population Estimates and Projections 1975). Where possible, the site-dependent parameters were taken from the environmental statements issued for each reactor (Table 3 ). The generic parameters used for this study such as consumption rates, occupancy factors and holdup times are given in Tables A-I and A-2 below. It should be noted that generic consumption rates for aquatic foods and inhalation rates are taken from Regulatory Guide I.109 (NRC 1977). Bioaccumulation factors and terrestrial food transfer factors were taken from Regulatory Guide 1.109 (NRC 1977). Dose commitment factors for the four age groups were taken from Hoenes and Soldat (1977).

TABLE A-1. Generic Consumption Rates and Occupancy Factors Used for the Study of Average Members of the Population (a)

\begin{tabular}{|c|c|c|c|c|}
\hline Pathway & Infant & Child & Teen-ager & AduTt \\
\hline $\begin{array}{l}\text { Fruits, vegetables and grain } \\
\quad(\mathrm{kg} / \mathrm{yr})\end{array}$ & 0 & 200 & 240 & 190 \\
\hline $\operatorname{Milk}(\mathrm{L} / \mathrm{yr})$ & 170 & 170 & 200 & 110 \\
\hline Meat and poujtry $(\mathrm{kg} / \mathrm{yr})$ & 0 & 37 & 59 & 95 \\
\hline Fish $(\mathrm{kg} / \mathrm{yr})^{(\delta)}$ & 0 & 2.2 & 5.2 & 6.9 \\
\hline Invertebrates $(\mathrm{kg} / \mathrm{yr})$ & $0(a)$ & 0.33 & 0.75 & 1.0 \\
\hline Drinking water (L/yr) & $170^{(c)}$ & 260 & 260 & 370 \\
\hline Inhalation $\left(\mathrm{m}^{3} / \mathrm{yr}\right)$ & $1400^{(d)}$ & 3700 & 8000 & 8000 \\
\hline $\begin{array}{l}\text { Air submersion and ground } \\
\text { irradiation occupancy } \\
\text { factor }\end{array}$ & 0.5 & 0.5 & 0.5 & 0.5 \\
\hline
\end{tabular}

(a) Regulatory Guide 1.109 (NRC 1977).

(b) Both fresh and salt water.

(c) Assumed to be equal to milk consumption.

(d) Same as for maximum individual. 
TABLE A-2. Holdup Times Between Harvest and Food

Holdup Time (days)

Fruits, grains and vegetables Milk

14

Meat ${ }^{(b)}$

Aquatic foods (fish and invertebrates)

Drinking Water

(a) Regulatory Guide 1.109 (NRC 1977)

(b) Value given is time after mitking or slaughter. For the portion of the time animals were fed stored feed, an additional 90 days was added to the holdup time.

SOURCE TERMS

The doses were estimated using the measured relepses as reported by the site operators for 1984 (Tichler, et al. 1987). ${ }^{\text {a) The releases }}$ include all radionuclides specified by the NRC to be measured and reported by the operators of all commercial nuclear power plants. Radionuclides given as a combination of parent-daughter isotopes such as $\mathrm{Y} / \mathrm{Sr}-90, \mathrm{Zr} / \mathrm{Nb}-95, \mathrm{Ba} / \mathrm{La}-140, \mathrm{I} / \mathrm{Xe}-133$ and $\mathrm{Pr} / \mathrm{Ce}-144$ were divided evenly between the parent and daughter.

The radionuclides used in this study, along with their half-lives, are given in Table A-3. Note that the " $+D^{n}$ after some of the nuciides indicates that the decay energy of the daughter is included with the parent. Thus, whenever a parent nuclide release is specified, the result of the dose calculation is as though an additional equilibrium amount of the daughter nuclide is specified. The daughter nuclide itself is included separately if it can be released independently of the parent and/or if it has a relatively long half-life.

(a) Very shortlived isotopes such as $\mathrm{Kr}-90,91,93,94, \mathrm{Xe}-139,140,141$, 143 and Rb-88M; those not likely to be produced; and those that were daughters whose decay energies were accounted for in the dose factor for the parent were not included in the close. 
TABLE A-3. Radionuclides Considered in This Study

\begin{tabular}{|c|c|c|c|c|c|}
\hline №. & Nuclide & $\begin{array}{c}\text { Decay Constant } \\
(1 / \text { sec })\end{array}$ & No. & Nuclide & $\begin{array}{l}\text { Decay Constant } \\
\quad(1 / \mathrm{sec})\end{array}$ \\
\hline 1 & $\mathrm{H}-3$ & $1.78 \mathrm{E}-09$ & 43 & $N b-97$ & $1.57 E-04$ \\
\hline 2 & $\mathrm{Be}-10$ & $1.37 \mathrm{E}-14$ & 44 & Mo-99+D & $2.92 \mathrm{E}-06$ \\
\hline 3 & $C-14$ & $3.83 \mathrm{E}-12$ & 45 & TC-99M & $3.19 \mathrm{E}-05$ \\
\hline 4 & $\mathrm{~N}-13$ & $1.16 \mathrm{E}-03$ & 46 & $R u-103+D$ & $2.02 E-07$ \\
\hline 5 & $F-18$ & $1.05 \mathrm{E}-04$ & 47 & $R u-106+D$ & $2.17 \mathrm{E}-08$ \\
\hline 6 & $\mathrm{Na}-22$ & $8.44 \mathrm{E}-09$ & 48 & $A g-110 M+D$ & 3.19E-08 \\
\hline 7 & $\mathrm{Na}-24$ & $1.28 \mathrm{E}-05$ & 49 & Cd-II5M & $1.80 \mathrm{E}-07$ \\
\hline 8 & Ar-4I & $1.05 \mathrm{E}-04$ & 50 & $C d-115$ & $3.60 E-06$ \\
\hline 9 & Sc-46 & $9.58 \mathrm{E}-08$ & 51 & $S n-125+D$ & $8.31 E-07$ \\
\hline 10 & $\mathrm{Cr}-51$ & $2.89 E-07$ & 52 & $5 b-124$ & $1.33 \mathrm{E}-07$ \\
\hline 11 & $M n-54$ & $2.57 \mathrm{E}-08$ & 53 & $S b-125+0$ & $8.06 \mathrm{E}-09$ \\
\hline 12 & $M n-56$ & $7.47 E-05$ & 54 & $T e-132+D$ & $2.47 E-06$ \\
\hline 13 & $\mathrm{Fe}-55$ & $8.14 \mathrm{E}-09$ & 55 & Te-133M+0 & $2.09 E-04$ \\
\hline 14 & $\mathrm{Fe}-59$ & 1.80E-07 & 56 & $I-13 I+0$ & $9.97 \mathrm{E}-07$ \\
\hline 15 & $\mathrm{Co}-57$ & $2.97 \mathrm{E}-08$ & 57 & $1-132$ & $8.42 E-05$ \\
\hline 16 & $\mathrm{Co-58}$ & $1.12 \mathrm{E}-07$ & 58 & $I-133+0$ & $9.25 E-06$ \\
\hline 17 & Co-60 & $4.17 \mathrm{E}-09$ & 59 & I-134 & $2.20 E-04$ \\
\hline 18 & N. i -57 & $5.35 E-06$ & 60 & $\mathrm{I}-135+\mathrm{D}$ & $2.92 \mathrm{E}-05$ \\
\hline 19 & $\mathrm{Ni}-63$ & $2.20 E-10$ & 61 & $X e-131 M$ & $6.69 \mathrm{E}-07$ \\
\hline 20 & $\mathrm{Ni}-65$ & $7.64 \mathrm{E}-05$ & 62 & $X e-133 M$ & $3.61 \mathrm{E}-06$ \\
\hline 21 & $\mathrm{Cu}-64$ & $1.52 \mathrm{E}-05$ & 63 & $x e-133$ & $1.52 \mathrm{E}-06$ \\
\hline 22 & $2 n-65$ & $3.31 \mathrm{E}-08$ & 64 & $\mathrm{Xe}-135 \mathrm{M}$ & $7.56 \mathrm{E}-04$ \\
\hline 23 & $Z n-69 M+D$ & $1.39 \mathrm{E}-05$ & 65 & $x e-135$ & $2.10 \mathrm{E}-05$ \\
\hline 24 & As -76 & $7.32 \mathrm{E}-06$ & 66 & $x e-137$ & $3.01 \mathrm{E}-03$ \\
\hline 25 & $\mathrm{Br}-82$ & $5.44 \mathrm{E}-06$ & 67 & $x e-138+0$ & $8.14 \mathrm{E}-04$ \\
\hline 26 & $\mathrm{Kr}-83 \mathrm{M}$ & $1.04 \mathrm{E}-04$ & 68 & $C_{5}-134$ & $1.07 \mathrm{E}-08$ \\
\hline 27 & $\mathrm{Kr}-85 \mathrm{M}$ & $4.31 \mathrm{E}-05$ & 69 & $C_{5}-136$ & $6.17 \mathrm{E}-07$ \\
\hline 28 & $\mathrm{Kr}-85$ & $2.05 E-09$ & 70 & $C s-137+D$ & $7.31 \mathrm{E}-10$ \\
\hline 29 & $\mathrm{Kr}-87$ & $1.52 \mathrm{E}-04$ & 71 & Cs-138 & $3.58 \mathrm{E}-04$ \\
\hline 30 & $\mathrm{Kr}-88+0$ & $6.89 \mathrm{E}-05$ & 72 & $C 5-139+D$ & $1.24 \mathrm{E}-03$ \\
\hline 31 & $\mathrm{Kr}-89$ & $3.64 \mathrm{E}-03$ & 73 & $\mathrm{Ba}-139$ & 1.39E-04 \\
\hline 32 & $R b-88$ & $6.53 \mathrm{E}-04$ & 74 & $\mathrm{Ba}-140+\mathrm{D}$ & $6.28 \mathrm{E}-07$ \\
\hline 33 & $R b-89+D$ & $7.61 \mathrm{E}-04$ & 75 & La-140 & $4.78 \mathrm{E}-06$ \\
\hline 34 & $S r-89+D$ & $1.59 \mathrm{E}-07$ & 76 & La-141 & 4. $97 \mathrm{E}-05$ \\
\hline 35 & $5 r-90+D$ & $7.58 \mathrm{E}-10$ & 77 & Ce-14I & $2.47 \mathrm{E}-07$ \\
\hline 36 & $S r-91+D$ & $2.03 E-05$ & 78 & $\mathrm{Ce}-144+0$ & $2.83 E-08$ \\
\hline 37 & $S r-92+D$ & $7.11 E-05$ & 79 & Eu-152 & $1.69 \mathrm{E}-09$ \\
\hline 38 & $Y-90$ & $3.01 \mathrm{E}-06$ & 80 & Eu-154 & $2.55 \mathrm{E}-09$ \\
\hline 39 & $Y-91 M+D$ & $2.32 \mathrm{E}-04$ & 81 & $W-187$ & $8.06 E-06$ \\
\hline 40 & $2 r-95+D$ & $1.22 \mathrm{E}-07$ & 82 & Th $-232+D$ & $1.57 \mathrm{E}-18$ \\
\hline 41 & $2 r-97+0$ & $1.14 \mathrm{E}-05$ & B3 & $N p-239$ & $3.42 E-06$ \\
\hline 42 & Nb-95 & $2.29 \mathrm{E}-07$ & & & \\
\hline
\end{tabular}


METEOROLOGY

When more than one set of meteorological (joint frequency) data was available for a site, the one which appeared to be the most reliable was used to generate atmospheric transport factors. Factors were calculated for 16 compass points and for ten radii from 2 to $80 \mathrm{~km}$ (see Table A-4) using the NRC computer program XOQDOQ (Sagendorf 1977).

TABLE A-4. Radius Intervals and Midpoints for Airborne Dose Calculations $(\mathrm{km})$

\begin{tabular}{cc} 
Interval & Midpoint \\
\hline $2-3$ & 2.5 \\
$3-4$ & 3.5 \\
$4-6$ & 5 \\
$6-9$ & 7.5 \\
$9-14$ & 11.5 \\
$14-20$ & 17 \\
$20-30$ & 25 \\
$30-40$ & 35 \\
$40-60$ & 50 \\
$60-80$ & 70
\end{tabular}

The $\times 0 Q 00 Q$ program generates four sets of atmospheric transport factors:

- average annual atmospheric dilution factors, which are not corrected for cloud depletion or radioactive decay

- dilution factors that are onty corrected for decay assuming a 2.26-day half-life

- dilution factors that are corrected for depletion and for decay assuming an 8-day half-life

- relative deposition per unit area.

These factors were used to estimate the dose from airborne releases using methods similar to the NRC GASPAR program (Eckerman, et al. 1980). The 
transport factors used this year were the same as those used for the previous estimates. The assumptions used in the calculation of these transport factors were as follows:

- 50-m source height with no correction for plume rise or building wake effects

- semi-infinite cloud model with sector-average, Gaussian-plume dispersion

- no correction for terrain height variation.

Since information about height and locations at each site for the releases given in Tichler, et al. (1987) was unavailable, a single generic height of $50 \mathrm{~m}$ was used at each site for the release point. Because the heights and locations of releases are uncertain, estimates of dose to persons living within $2 \mathrm{~km}$ of the site could be in serious error; only persons living between 2 to $80 \mathrm{~km}$ from the site were included in the dose estimates.

POPULATION

The population distribution within 2 to $80 \mathrm{~km}$ around each site was determined from information supplied by the NRC from an updated reduction of 1980 census data (Sinisgalli 1982). Also the NRC supplied updated estimates of the number of people residing in major metropolitan centers within the $80-\mathrm{km}$ region around each site (Brauner 1982) and D. P. Cleary.

FOOD PRODUCTION VERSUS FOOD CONSUMPTION

The total food production for the region within $80 \mathrm{~km}$ around each site was the product of the NRC state-wide productivity figure for each state and a site productivity factor. At some sites this total production may be more or less than the total consumption; i.e., population times average individual consumption (see Table A-1 for generic consumption rates). When production was more than consumption for a site, it was assumed that all persons in the $2-t 0-80-\mathrm{km}$ region ate contaminated food; when production was less than consumption, it was assumed that dilution would occur because uncontaminated food would be shipped into the area from outside. Thus, the calculated doses for a particular food type were reduced in proportion to the ratio of production divided by consumption (production/consumption less than 1 ).

(a) Letter from D. P. Cleary, U.S. Nuclear Regulatory Commission, to D. A. 8aker, Pacific Northwest Laboratory, June 1987. 
The dose to persons outside the $80-\mathrm{km}$ Timit from food shipped out of the region, in the case of production being greater than consumption, is not included in this report because we are concerned only with the dose within the $80-\mathrm{km}$ radius. These production/consumption factors are given for reference as footnotes to the tables showing airborne dose cormitment in the Site Surmaries section.

\section{DRINKING WATER}

The population between 2 and $80 \mathrm{~km}$ distance from each plant site exposed to drinking water contaminated with released radionuclides was generally obtained from the environmental statement (ES) for the plant. For all sites located on salt water, it was assumed that no dose was received from drinking water. The generic consumption rates used for drinking water are given in TabTe A-1.

The radionuclide concentration in the drinking water consumed by a population downstream from a site was usually estimated assuming $100 \%$ mixing of the plant effluent with the river. For lakes, an overall dilution factor was estimated from dilution factors given in the ES for each population center along the shore (within $80 \mathrm{~km}$ ) that consumes the contaminated Take water. These individual factors were weighted by population and averaged to obtain an effective dilution factor for the total population exposed to contaminated drinking water.

\section{AQUATIC FOOD}

Wherever possible, the fish-catch data from the plant ES were used to estimate aquatic food consumption rates for the population living within the region. When these data were not found in the ES or were considered unrealistic, the generic values of Table A-1 were used.

The average radionuclide concentration of the waters in which this food was harvested was estimated assuming an additional dilution over the effluent flow from the reactor. For rivers, it was assumed that the fish were caught in waters in which the plant effluent was completely diluted. For Takes, an additional factor as given in the ES was used; when none was given in the ES, a generic value of 0.01 was used. For ocean and bay sites, generic values of 0.001 and 0.002 were used for fish and invertebrates, respectively, if the ES yielded no values for these parameters. Invertebrates were not assumed to be caught in sufficient 
quantity at freshwater sites (river and lake) to affect the population dose and therefore were not inciuded in the dose calculation. Any exceptions to these general guidelines are explained in the footnotes to the individuat site surmaries.

TECHNICAL NOTES

The calculations leading to the dose estimates contained in this report were generated from recent versions of computer programs originally documented in Baker et a1. (1977). The revised programs were written in BASICA and run on an IBM PC microcomputer operating under the PC-DOS operating system. The charts were alt made using an HP LaserJet II printer. 
APPENDIX REFERENCE

Baker, D. A., J. K. Soldat and E. C. Watson. 1977. Population-Dose Commitments Due to Radioactive Releases from Nuclear Power Plant Sites in 1975. PNL-2439, Pacific Northwest Laboratory, Richland, Washington.*

Brauner, A. 1982. Population Estimates, Nuclear Power PTant Nearby Population Concentrations. U.S. Nuctear Regulatory Commission, Washington, D.C.

Eckeman, K. F., F. J. Congel, A. K. Roecklein and W. J. Pasciak. 1980. Users Guide to GASPAR Code. NUREG-0597, U.5. Nuclear Regulatory Commission, Washington, D.C.

Hoenes, G. R., and J. K. Soldat. 1977. Age-Specific Radiation Dose Commitment Factors for a One-Year Chronic Intake. NUREG-0172, U.S. Nuctear Regulatory Comission, Washington, T..C.*

Population Estimates and Projections, Current Population Reports. 1975. Series P-25, No. 541. U.S. Department of Conturerce, Social and Economic Statistics Administration, Bureau of the Census.

Sagendorf, J. F., and J. T. Goll. 1977. X0QDOQ--Program for the Meteorological Evaluation of Routine EffTuent Releases at NuTcear Power Stations. NUREG-0324, 0.S. Nuclear Regulatory Commission, Washington, D.C. ${ }^{\star *}$

Sinisgalli, A. 1982. 1980 Residential Population Estimates, 0-80 Kilometers for Nuclear Power Plants. U. S. Nuclear ReguTatory Conmission, Washington, D.C.

Statistical Abstract of the United States: 1980. 101st edition. U.S. Department of Commerce, Bureau of the Census, Washington, D.C.

Tichler, J., K. Norden, and J. Congemi. 1987, Radioactive MateriaTs Released from Nuclear Power Plants, Annual Report 1984. NUREG-CR-2907, BNL-NUREG-51581, VoT. 5, U.S. Nuclear ReguTa tory Comiss sion, Washington, D.C.*

U.S. Nuclear Regulatory Commission. 1977. Calculation of Annual Doses to Man from Routine Releases of Reactor Effluents for the Purpose of Evaluating Compliance with 10 CFR Part 50, hppendix I, Revision 1. Regulatory Guide 1.109, Washington, D.C.

*Available for purchase from the National Technical Information Service, Springfield, va 22161.

* Available free upon written request to the Division of Technical Information and Document Control, U.S. Nuclear Regulatory Commission, Washington, DC 20555 . 
No. of

Copies

OFFSITE

U.S. Nuclear Regulatory Commission

Division of Technical Information and Document Control

7920 Norfolk Avenue

Bethesda, MD 20014

W. E. Bolch

University of Florida

Department of Environmental

Engineering Sciences

AP Black Hall

Gainesville, FL 32611

F. J. Borst

Fort St. Vrain Nuclear

Generating Station

16805 Weld County Road $191 / 2$

Platteville, c0 80651

K. R. Brautigam

Abteilung fur Angewandte Systemanalyse (AFAS)

Kernsforschungszentrum Karlsruhe

7514 Eggenste in - Leopoldshafen

Karlsruhe

Federal Republic of Germany

H. D. Brenk

Zentralabteilung Strahlenschulz der Kernforschungsantage Jül ich GmbH

Postfach 1913 - 5170 JuTich 1

Federal Republic of Germany

J. T. Brennen, M.D.

Radiation Management Corporation

3508 Market Street

Philadelphia, PA 19104

R. M. DeNuTman

Instituto Naciona 7 De

Investigaciones Nucleares

Benjamin Frank in 161

MEXICO 11, D. F.
No. of

Copies
T. Dipierro

Building 475

Brookhaven National Laboratory

Upton, NY 11973

P. J. Dostie

State of Maine

Department of Human Services

Augusta, ME 04333

D. E. Dunning, Jr.

Evaluation Research Corporation 800 0ak Ridge Turnpike, Suite 501

Oak Ridge, TN 37830

K. F. Eckerman

Oak Ridge National Laboratory

P.0. Box $X$

Oak Ridge, TN 37830

Ecological Sciences Information Center

Oak Ridge National Laboratory

Oak Ridge, TN 3783D

R. J. Feinberg

Knolis Atomic Power Laboratory

General Electric Company

Box 1072

Schenectady, NY 12301

B. Feldman

Energy Systems Research Group

$120 \mathrm{Milk}$ Street

Boston, MA D2107

J. W. Ferman

Division of Water Quality

Minnesota Pollution Control Agency

1935 West County Road B-2

Roseville, MN 55113

J. F. Greene

Mecklenburg County Department of Environmental Health

1200 Blythe Boulevard

Charlotte, NC 282D3 
NUREG/CR-2850

PNL-4221

Vol. 6

No. of

Copies

40 R. A. Hartfield

Office of Administration and Resources Management

Nuclear Regulatory Commission

Washington, DC 20555

J. Hennigan

Division of Radiation Health Bureau of Environmental Health Michigan Department of Public Health 3500 North Logan

Lansing, MI 48906

E. Hewitt

FMC Corporation

Box 4111

PocatelTo, ID 83202

F. 0. Hoffman

Oak Ridge National Laboratory

P.0. Box $X$

Oak Ridge, TN 37830

L. Huebner

Hazleton Environmental Sciences

1500 Frontage Road

Northbrook, IL 60062

A. P. HulT

Brookhaven National Laboratory

Upton, NY 11973

J. Iacovino

Nuclear Safety and Licensing

Westinghouse Electic Corporation

Box 355

Pittsburg, PA 15230

C. Johnson, M.D.

42 Hillside Drive

Denver, CO 80215

M. L. Joshi

Government of India

Department of Atomic Energy

Rajasthan Atomic Power Project

Nuclear Training Centre

INDIA
No. of

Copies

B. Kahn

Environmental Resources Center

Georgia Institute of Technology

Atlanta, GA 30332

M. Laraia

C.N.E.N., Oisp/Centr

00198 Roma

Viale Regina Margherita, 125

ITALY

L. Lewis

Duke Power Company

42? S. Church Street

Charlotte, NC 28242

S. Mattsson

Lunds Universitet

Radiofysiska Institutionen

Lasarettet, S-221 85 Lund

SWEDEN

H. R. Meyer

Oak Ridge National Laboratory

Oak Ridge, TN 37830

S. Morris

Building 475

Brookhaven NationaT Laboratory

Upton, NY 11973

J. Nagy

Bldg. 475

Brookhaven National Laboratory Upton, NY 11973

L. Namestek, Ing.

Prager Gasse $11 / 5$

A-3002 Purkersdorf

AUSTRIA

K. K. Narayanan

Bhabha Atomic Research Centre

Bombay - 400085

INOIA 
No. of

Copies

Y. C. Ng

Lawrence Livermore National

Laboratory

University of California

P.0. Box 808

Livermore, CA 94550

W. $0 \mathrm{gg}$

PSD CRBRP

P.0. Box U

Oak Ridge, TN 37830

F. E. Owens

Washington Public Power Supply System

George Washington Way

Richiand, WA 99352

C. Pomroy

Health \& Welfare Canada

Health Protection Branch

Radiation Protection Bureau

Ottawa, K1A 1CI

CANADA

\section{G. Poretti}

ABT. F. MEDIZ. Strahienphysik Inselspital

CH-3010 BERN/SWITZERLAND

\section{J. W. Poston}

School of Nuclear Engineering Georgia Institute of Technology Atlanta, GA 30332

R. R. Roselius

Consumers Power Company

Midland Plant Operations

P.0. Box 1593

Midland, MI 48640

P. Ross

Congressional Information Service 4520 East West Highway

Washington, DC 20014
No. of

Copies

\author{
V. Sailor \\ Building 701 \\ Brookhaven National Laboratory \\ Upton, NY 11973 \\ J. Tadmor \\ Director of Research and \\ Coordinator \\ Advisory Committee for \\ Nuclear Safety \\ Soreq Nuclear Research Centre \\ Yavne, ISRAEL \\ T. Thomas \\ National Institutes of Health \\ Landow Building, Room 3C16 \\ 9000 Rockville Pike \\ Bethesda, MD 20014 \\ A. N. Tshaeche \\ Mail 682 \\ General Electric Company \\ 175 Curtner Avenue \\ San Jose, CA 95125 \\ F. Valerio \\ Universita $0 i$ Genova \\ Instituto Di Oncologia \\ 16132 Genova \\ Viale Benedetto XV N. 10 \\ ITALY \\ P. Vasudev \\ Environment Canada \\ Place Vincent Massey \\ 45th Floor \\ 0ttawa, KIA 1 C8 \\ CANADA \\ G. H. Whipple \\ 3301 Rutland Loop \\ TatTahassee, FL 32312 \\ E. I. White \\ 7712 Parma Lane \\ San Diego, CA 92126
}


No. of

Copies

F. E. Yost

Utility Data Institute, Inc.

2011 I Street, NW, Suite 700

Washington, DC 20006

ONSITE

2 Westinghouse Hanford Company

H. R. Brager

P. R. Prevo

25 Pacific Northwest Laboratory

D. A. Baker (10)

C. E. Elderkin

D. W. Dragnich

M. J. Graham

J. M. HaTes

P. C. Hays

T. L. Page

R. A. Peloquin

R. G. Schreckhise

R. E. Wildung

Publishing Coordination (2)

Technical Information (5) 


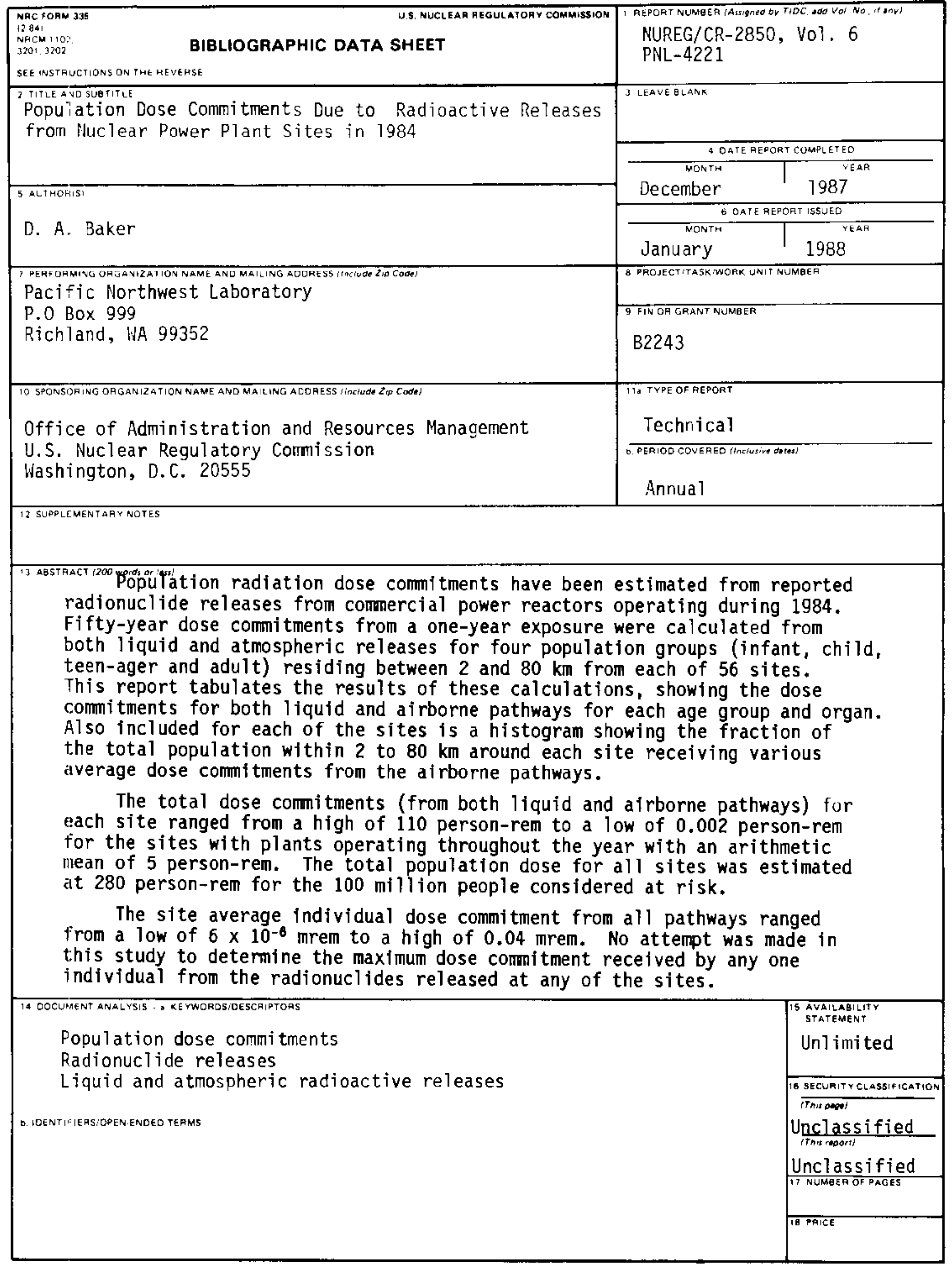


